RODRIGO CUTRI

MÉTODO DE EXTRAÇÃO EM TEMPO REAL DE SEQÜÊNCIA POSITIVA, NEGATIVA E/OU HARMÔNICOS

SÃo PAULO

2008 
RODRIGO CUTRI

\section{MÉTODO DE EXTRAÇÃO EM TEMPO REAL DE SEQÜÊNCIA POSITIVA, NEGATIVA E/OU HARMÔNICOS}

Tese apresentada à Escola

Politécnica da Universidade de

São Paulo para obtenção do

Título de Doutor em Engenharia

Área de concentração :

Engenharia Elétrica

Orientador :

Prof. Dr. Lourenço Matakas Junior

SÃO PAULO

2008 
A Deus

A meus pais e minha irmã

À minha noiva

A todos que me apoiaram com seus exemplos, gestos e palavras. 


\section{AGRADECIMENTOS}

Agradeço primeiramente a Deus, o Grande Arquiteto do Universo, por me guiar, concedendo forças para nunca desistir e fazendo-me perseverar acreditando sempre.

Agradeço a todos que colaboraram para que esta tese se realizasse, em especial meus pais, minha irmã e minha noiva (agora sim, poderemos nos casar). Agradeço a compreensão, a paciência e o carinho em todos os momentos.

Agradeço em especial ao professor Lourenço Matakas Jr. pelo companheirismo, pela dedicada orientação que contribuiu para que eu pudesse aprimorar minha forma de pensar, de expressar idéias e pesquisar problemas, descobrir suas causas, analisar e propor soluções. Desde o início do mestrado, são quase sete anos de encontros de idéias e orientações. Agradeço também aos professores Walter Kaiser e Wilson Komatsu pelas sugestões que colaboraram para o aperfeiçoamento deste trabalho.

Agradeço ao professor Sigmar Maurer Deckmann da UNICAMP que participou de minha banca de mestrado e cuja discussão técnica foi muito importante para o desenvolvimento deste trabalho.

Agradeço a Universidade de São Paulo e a Escola de Engenharia Mauá pela formação e apoio.

Aos professores Mário Pagliaricci, Nilson De Lucca e Jorge Janiszewski pelo exemplo, pela amizade, colaboração e incentivo em vários momentos desta pesquisa. 


\section{RESUMO}

Harmônicos e desequilíbrios podem causar uma série de problemas a equipamentos conectados a rede elétrica. Este trabalho propõe um método de extração em tempo real das componentes de seqüência positiva, negativa e/ou harmônicos que não requer transformações de coordenadas ou filtragem de sinais. O método proposto é verificado via simulação numérica e implementado experimentalmente em um DSP. Como exemplos de aplicação apresentam-se: a simulação de um Filtro Ativo de Potência utilizando-se um conversor do tipo fonte de tensão operando em PWM e um Relé de Seqüência Negativa.

Palavras-chave: Engenharia Elétrica. Sistemas de Potência. Qualidade de Energia Elétrica. Sequências Positiva, Negativa e Zero. 


\begin{abstract}
Harmonics and unbalances cause several problems to equipments connected to AC mains. This work proposes a real time method to obtain the positive and negative sequence components and/or harmonics that requires no coordinate transformation neither signal filtering. Numerical simulation and experimental results are shown to validate the proposed method. As examples of application an Active Power Filter using a voltage source converter operating with PWM and an Negative Sequence Power Relay are presented.
\end{abstract}

Keywords: Electrical Engineering. Power Systems. Power Quality. Positive, Negative and Zero Sequence. 


\section{LISTAS DE FIGURAS}

Fig. 1

Fig.2-1

Fig. 2.1-1

Fig. 2.1.1-1

Fig. 2.1.1-2

Fig. 2.1.1-3

Fig. 2.1.1-4

Fig. 2.1.2-1

Fig. 2.1.2-2

Fig. 2.1.2-3

Fig. 2.1.2-4

Fig. 2.1.2-5

Fig. 2.2-1

Fig. $3 a$

Fig. 3b

Fig. 3c

Fig. 3d

Fig.4.1.1a

Fig. $4.1 .1 \mathrm{~b}$

Fig.4.1.2a
- Diagrama de Blocos - Estrutura da Tese

- Diagrama de Blocos - Métodos de extração das seqüências positiva, negativa e/ou harmônicos

- Vetor espacial $\vec{S}$, e os sistemas de coordenadas rst e $\alpha \beta$

- Vetor $\vec{S}$ e sua trajetória (pontilhada)

- Projeção do Vetor $\vec{S}$ no sistema $\alpha \beta$

26

- Projeção no sistema $\alpha \beta$ do Vetor $\overrightarrow{S_{1 f}}$ após a 27 filtragem

- Diagrama de Blocos do Método DTRVE (extração seqüência negativa) sistema fixo de referência

- Vetores da componente fundamental representada em um sistema fixo e num sistema girante de referência

- Diagrama de Blocos do Método RSP

- Diagrama de Blocos do Método RNP

- Diagrama de Blocos do Método Proposto

- Diagrama em blocos do algoritmo de extração da componente de seqüência positiva da fundamental

- Diagrama em blocos do algoritmo de extração da componente de seqüência negativa da fundamental componente de seqüência negativa e harmônicos (extração total)

- Diagrama em blocos do algoritmo de extração dos harmônicos

- Sinal distorcido simulado $\left(1^{\circ}+-, 3^{\circ}+-, 5^{\circ}+-\right)$

- Sinal distorcido simulado $\left(1^{\circ}+-, 2^{\circ}+-, 4^{\circ}+-\right)$

- Sinal distorcido experimentalmente gerado através 
$\left(1^{0}+-, 3^{0}+-, 5^{0}+-\right)$

Fig.4.1.2b - Sinal distorcido experimentalmente gerado através

de matriz de pontos pré-programada

$\left(1^{\circ}+-, 2^{\circ}+-, 4^{0}+-\right)$

Fig.4.1.3a - Sinais extraídos - Método A (Simulação) - $\left(1^{\circ}-\right.$ , $\left.5^{\circ}+\right)$

Fig.4.1.3b - Sinais extraídos - Método A (Simulação) - $\left(1^{\circ+-}\right.$ , $\left.2^{\circ}+-, 4^{\circ}+-\right)$

Fig.4.1.4a - Sinais extraídos - Método A (Experimental) - $\left(1^{\circ}\right.$ , $\left.5^{\circ}+\right)$

Fig.4.1.4b - Sinais extraídos - Método A (Experimental) - (10+, $\left.2^{\circ}+-, 4^{\circ}+-\right)$

Fig.4.1.5a - Sinais extraídos - Método B (Simulação) - $\left(1^{\circ}\right.$ , $\left.3^{\circ}+, 5^{\circ}-\right)$

Fig.4.1.5b - Sinais extraídos - Método B (Simulação) - (10+, $\left.2^{\circ}+-, 4^{0}+-\right)$

Fig.4.1.6a - Sinais extraídos - Método B (Experimental) - (1º , $\left.3^{\circ}+, 5^{\circ}-\right)$

58

Fig.4.1.6b - Sinais extraídos - Método B (Experimental) - $\left(1^{\circ}+-\right.$

Fig.4.1.7a , $\left.2^{\circ}+-, 4^{\circ}+-\right)$ $\left(1^{\circ}+, 5^{\circ}-\right)$

Fig.4.1.7b - Sinais extraídos - Método C (Simulação) ) - (10+, $\left.2^{\circ}+-, 4^{0}+-\right)$

Fig.4.1.8a - Sinais extraídos - Método C (Experimental) ) $\left(1^{\circ}+, 5^{\circ}-\right)$

59

59

Fig.4.1.8b - Sinais extraídos - Método C (Experimental) ) $\left(1^{\circ}+-, 2^{0}+-, 4^{\circ}+-\right)$

Fig.4.1.9a - Sinais extraídos - Método D (Simulação) - $\left(1^{\circ}+, 3^{\circ}-\right.$ , $\left.5^{\circ}+\right)$

60

Fig.4.1.9b - Sinais extraídos - Método D (Simulação) - $\left(1^{\circ}+-\right.$ 60 , $\left.2^{\circ}+-, 4^{0}+-\right)$

Fig.4.1.10a - Sinais extraídos - Método D (Experimental) $\left(1^{\circ}+, 3^{\circ}-, 5^{\circ}+\right)$

Fig.4.1.10b - Sinais extraídos - Método D (Experimental) - $\left(1^{\circ}+-\right.$ , $\left.2^{\circ}+-, 4^{\circ}+-\right)$

Fig.4.1.11a - Sinais extraídos - Método AB (Simulação) - (1º-)

Fig.4.1.11b - Sinais extraídos - Método AB (Simulação) - (10+, $\left.2^{\circ}+-, 4^{\circ}+-\right)$ 
Fig.4.1.12a - Sinais extraídos - Método AB (Experimental) - (10-)

Fig.4.1.12b - Sinais extraídos - Método AB (Experimental) -

Fig.4.1.13a - Sinais extraídos - Método CD (Simulação) - $\left(1^{\circ+}\right)$

62

Fig.4.1.13b - Sinais extraídos - Método CD (Simulação) - ( $1^{\circ}+-$

Fig.4.1.14a , $\left.2^{\circ}+-, 4^{\circ}+-\right)$

Fig.4.1.14b - Sinais extraídos - Método CD (Experimental) $\left(1^{\circ}+-, 2^{\circ}+-, 4^{0}+-\right)$

62

- Sinais extraídos - Método CD (Experimental) $\left(1^{\circ}+\right)$

Fig.4.1.15a

- Seqüências dos sinais extraídos - (Teórico tabela I, cap.3)

Fig.4.1.16a

- Seqüências dos sinais extraídos - (Simulado)

63

Fig.4.1.17a

- Seqüências dos sinais extraídos - (Experimental)

63

Fig.4.1.15b

- Seqüências dos sinais extraídos - (Teórico tabela II, cap.3)

Fig.4.1.16b

- Seqüências dos sinais extraídos - (Simulado)

63

64

Fig.4.1.17b

- Seqüências dos sinais extraídos - (Experimental)

64

Fig.4.2.1a

- Sinal distorcido para análise do transitório (Simulação)

Fig.4.2.1b

- Sinal distorcido para análise do transitório (Experimental)

Fig.4.2.2a - Sinais extraídos - Método A (Simulação) - Análise Transitória

64

66

66

66

Fig.4.2.2b - Sinais extraídos - Método A (Experimental) Análise Transitória

66

Fig.4.2.3a - Sinais extraídos - Método B (Simulação) - Análise Transitória

Fig.4.2.3b - Sinais extraídos - Método B (Experimental) Análise Transitória

Fig.4.2.4a - Sinais extraídos - Método C (Simulação) - Análise Transitória

Fig.4.2.4b - Sinais extraídos - Método C (Experimental) Análise Transitória

Fig.4.2.5a - Sinais extraídos - Método D (Simulação) - Análise Transitória

Fig.4.2.5b - Sinais extraídos - Método D (Experimental) Análise Transitória

Fig.4.2.6a

- Sinais extraídos - Método AB(Simulação) Análise Transitória 
Fig.4.2.6b - Sinais extraídos - Método AB(Experimental) -

Fig.4.2.7a - Sinais extraídos - Método CD(Simulação) -

Fig.4.2.7b - Sinais extraídos - Método CD(Experimental) 69

Fig. 4.3.1a - Operação A - Resposta em Freqüência para 72 vários harmônicos (impares) e seqüências. Acima: ganho Abaixo:fase

Fig. 4.3.1b - Operação A - Resposta em Freqüência para 73 vários harmônicos (pares) e seqüências. Acima: ganho Abaixo:fase

Fig. 4.3.2a - Operação B - Resposta em Freqüência para vários harmônicos (impares) e seqüências. Acima: ganho Abaixo:fase

Fig. 4.3.2b - Operação B - Resposta em Freqüência para vários harmônicos (pares) e seqüências. Acima: ganho Abaixo:fase

Fig. 4.3.3a - Operação C - Resposta em Freqüência para vários harmônicos (impares) e seqüências. Acima: ganho Abaixo:fase

Fig. 4.3.3b - Operação C - Resposta em Freqüência para vários harmônicos (pares) e seqüências. Acima: ganho Abaixo:fase

Fig. 4.3.4a - Operação D - Resposta em Freqüência para vários harmônicos (impares) e seqüências. Acima: ganho Abaixo:fase

Fig. 4.3.4b - Operação D - Resposta em Freqüência para vários harmônicos (pares) e seqüências. Acima: ganho Abaixo:fase

Fig. 4.3.5a - Operação $A B$ - Resposta em Freqüência para vários harmônicos (impares) e seqüências. Acima: ganho Abaixo:fase

Fig. 4.3.5b - Operação $A B$ - Resposta em Freqüência para vários harmônicos (pares) e seqüências. Acima: ganho Abaixo:fase

Fig. 4.3.6a - Operação CD - Resposta em Freqüência para vários harmônicos (impares) e seqüências. Acima: ganho Abaixo:fase

Fig. 4.3.6b - Operação CD - Resposta em Freqüência para vários harmônicos (pares) e seqüências. Acima: ganho Abaixo:fase

Fig. 4.3.7a - Operação $A B$ - Resposta em Freqüência (PADRÃO ONS) para vários harmônicos (impares) 
Fig. 4.3.7b - Operação $A B$ - Resposta em Freqüência (PADRÃO ONS) para vários harmônicos (pares) e seqüências. Acima: ganho Abaixo:fase

Fig. 5.1-1 - Diagrama em blocos do compensador ativo de desequilíbrios

Fig.5.1-2 - Diagrama em bloco mostrando o conversor e as malhas de controle de tensão no barramento $\mathrm{CC}$ e da corrente CA

Fig.5.1-3 - Filtro Ativo (Método A)

88

89

92

Fig.5.1-4 - Filtro Ativo (Método Compensação Total) 92

Fig.5.1-5 - Comportamento da malha de corrente (fase $r$ ) (Método A)

Fig.5.2-1 - Simulação Relé de Seqüência Negativa 95 Comportamento da corrente da rede (gráfico superior) Comportamento do acionamento do relé (gráfico inferior)

Fig. 5.2-2 - Circuito do relé de seqüência negativa simulado (Circuito Geral)

Fig. 5.2-3 - Circuito do relé de seqüência negativa simulado (cálculo das seqüências positiva e negativa)

Fig. 5.2-4 - Circuito do relé de seqüência negativa simulado (cálculo das correntes eficazes)

Fig. 5.2-5 - Circuito do relé de seqüência negativa simulado (cálculo do grau de desequilíbrio e comparação com set-point)

Fig. AP-1 - Operação A - Resposta em Freqüência para vários harmônicos de seqüência zero.

Fig.B-1 - Circuito Teste do Buffer (Simulação)

Fig.B-2

- Sinais Bufferizados (Simulação)

Fig.B-3

- Diagrama de Blocos do ADMC-401

Fig.B-4

- Buffer digital

Fig. C-1 - Circuito-teste referente a todos os métodos

Fig. C-2 - Circuito simulado método $\mathrm{A}$

Fig. C-3 - Circuito do filtro ativo simulado (compensador completo)

Fig. C-4a - Conversor (parte 1)

Fig. C-4b - Conversor (parte 2)

Fig. C-5 - Malha de tensão

Fig. C-6 - Malha de corrente 


\section{LISTAS DE TABELAS}

Tabela I

Tabela II

Tabela III

Tabela IV

Tabela V

Tabela VI

Tabela VII

Tabela VIII

Tabela IX

Tabela X
- Ganho de amplitude (p.u.) e fase $\left(^{\circ}\right)$ das 37 operações $A, B, C, D, A B, C D$ para harmônicas ímpares de sequência positiva e negativa.

- Ganho de amplitude (p.u.) e fase $\left(^{\circ}\right)$ das 38 operações $A, B, C, D, A B, C D$ para harmônicas pares de sequência positiva e negativa.

- Quadro demonstrativo dos sinais extraídos operação A

- Quadro demonstrativo dos sinais extraídos operação B

- Quadro demonstrativo dos sinais extraídos operação A (com harmônicos)

- Quadro demonstrativo dos sinais extraídos 50 operação B (com harmônicos)

- Duração do Transitório em relação a um ciclo da fundamental (base $60 \mathrm{~Hz}$ )

- Tempo de execução de cada método 86 implementado no DSP ADM401

- Quadro resumo das características adotadas referentes ao compensador para a simulação completa do sistema

- Ganho de amplitude (p.u.) e fase $\left(^{\circ}\right)$ das

ímpares de seqüência zero. 


\section{LISTAS DE ABREVIATURAS E SIGLAS}

$\begin{array}{ll}\text { AC } & \text { - Alternating current (Corrente alternada) } \\ \text { CC } & \text { - Corrente Contínua } \\ \text { CA } & \text { - Corrente Alternada } \\ \text { DSP } & \text { - Digital Signal Processor (Processador Digital de Sinais) } \\ \text { PLL } & \text { - Phase Locked Loop } \\ \text { DTRVE } & \text { - Método da Detecção em Tempo Real através de Vetores Espaciais } \\ \text { FPB } & \text { - Filtro Passa Baixa } \\ \text { FPA } & \text { - Filtro Passa Alta } \\ \text { PQ } & \text { - Método da Potência Ativa e Reativa Instantânea } \\ \text { RSP } & \text { - Método da Referência Síncrona Girante Positiva } \\ \text { RSN } & \text { - Método da Referência Síncrona Girante Negativa } \\ \text { DSNI } & \text { - Método da Injeção Direta de Seqüência Negativa } \\ \text { IRF } & \text { - Referência Instantânea de Filtragem - Instantaneous Filter Reference } \\ \text { VSI } & \text { - Voltage Source Inverter (Inversor tipo fonte de tensão) } \\ \text { PWM } & \text { - Pulse Width Modulation (Modulação em largura de pulso) } \\ \text { PCC } & \text { - Point of common coupling (Ponto de acoplamento comum) } \\ \text { PI } & \text { - Proporcional-Integral (Controlador) }\end{array}$




\section{LISTAS DE SÍMBOLOS}

\begin{tabular}{|c|c|}
\hline $\mathrm{s}_{\mathrm{r}}(\mathrm{t}), \mathrm{s}_{\mathrm{s}}(\mathrm{t}), \mathrm{s}_{\mathrm{t}}(\mathrm{t})$ & sinais instantâneos no sistema rst \\
\hline $\mathrm{s}_{0}(\mathrm{t})$ & sinal instantâneo de seqüência zero \\
\hline$\vec{S}(t)$ & $\begin{array}{l}\text { vetor espacial associado as correntes instantâneas de um } \\
\text { sistema trifásico }\end{array}$ \\
\hline$S(t)$ & módulo do sinal instantâneo associado ao sistema \\
\hline$\theta(\mathrm{t})$ & fase do sinal instantâneo associado ao sistema \\
\hline$S_{p}$ & valor de pico do sinal \\
\hline $\overrightarrow{S_{1}}$ & $\begin{array}{l}\text { vetor espacial associado a fundamental do sinal instantâneo de } \\
\text { um sistema trifásico }\end{array}$ \\
\hline$S_{1}(t)$ & $\begin{array}{l}\text { módulo da fundamental de um sinal instantâneo associado ao } \\
\text { sistema }\end{array}$ \\
\hline $\overrightarrow{S_{1 f}}$ & $\begin{array}{l}\text { vetor espacial associado ao sinal fundamental instantâneo } \\
\text { filtrado }\end{array}$ \\
\hline$\phi(\mathrm{t})$ & $\begin{array}{l}\text { fase da fundamental de um sinal instantâneo associado ao } \\
\text { sistema }\end{array}$ \\
\hline$\vec{S}_{h}$ & $\begin{array}{l}\text { vetor espacial associado aos harmônicos de um sinal } \\
\text { instantâneo de um sistema trifásico }\end{array}$ \\
\hline$S_{h}(t)$ & $\begin{array}{l}\text { módulo da harmônica de ordem } \mathrm{h} \text { de um sinal instantâneo } \\
\text { associado ao sistema }\end{array}$ \\
\hline$\phi_{\mathrm{h}}(\mathrm{t})$ & $\begin{array}{l}\text { fase da harmônica de ordem } \mathrm{h} \text { um sinal instantâneo associado } \\
\text { ao sistema }\end{array}$ \\
\hline$\omega$ & velocidade angular da rede \\
\hline h & ordem do harmônico \\
\hline $\overrightarrow{S_{+}}$ & $\begin{array}{l}\text { vetor espacial associado a fundamental de seqüência positiva de } \\
\text { um sinal de um sistema trifásico }\end{array}$ \\
\hline $\overrightarrow{S_{-}}$ & $\begin{array}{l}\text { vetor espacial associado a fundamental de seqüência negativa } \\
\text { de um sinal de um sistema trifásico }\end{array}$ \\
\hline
\end{tabular}




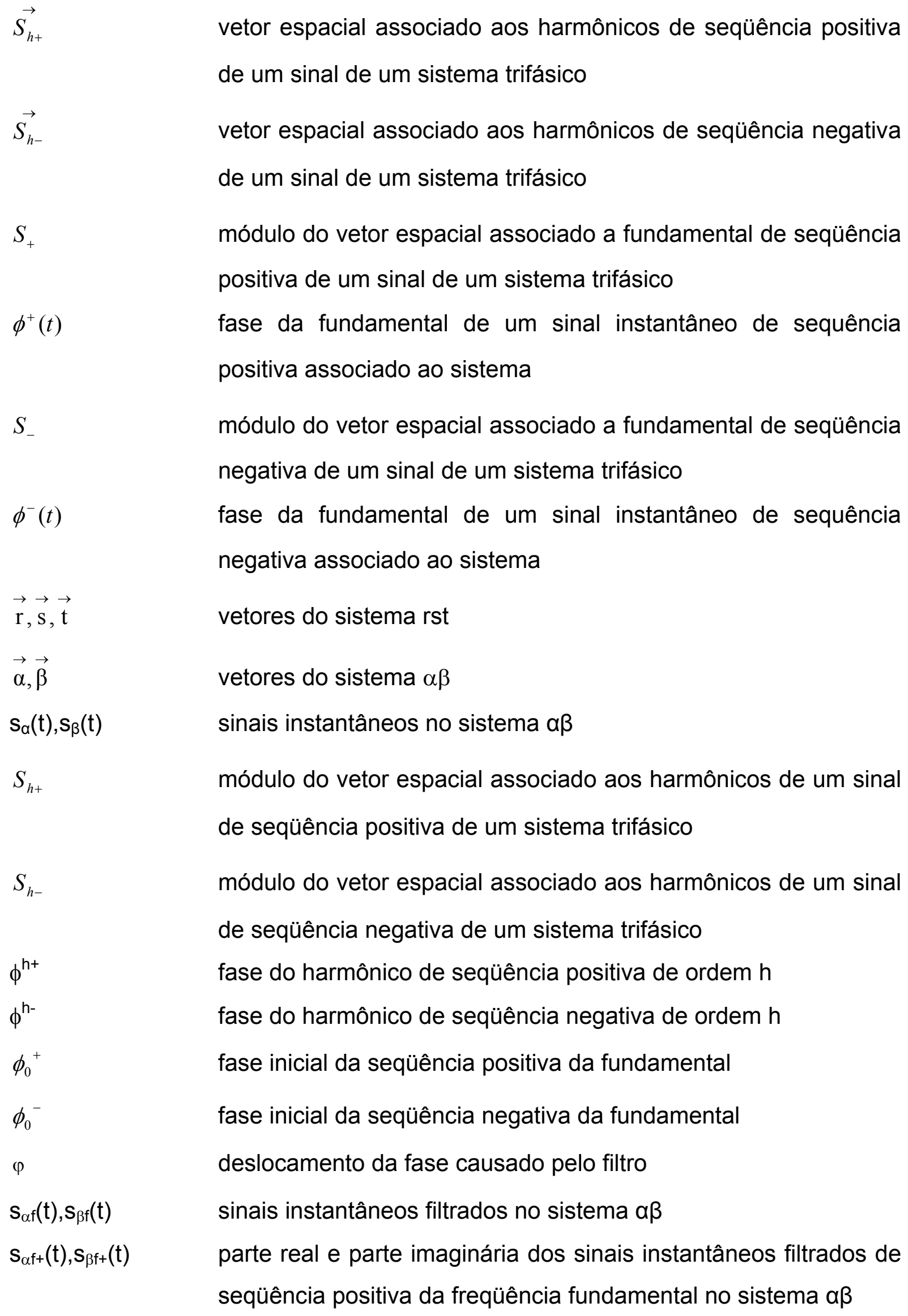




$$
\begin{aligned}
& \mathrm{s}_{\alpha \mathrm{f}-\mathrm{t}}(\mathrm{t}), \mathrm{s}_{\beta \mathrm{f}}(\mathrm{t}) \quad \text { parte real e parte imaginária dos sinais instantâneos filtrados de } \\
& \text { seqüência negativa da freqüência fundamental no sistema } \alpha \beta \\
& \mathrm{S}_{\alpha f 90}(\mathrm{t}), \mathrm{S}_{\beta \mathrm{f} 90}(\mathrm{t}) \quad \text { parte real e parte imaginária dos sinais instantâneos filtrados no } \\
& \text { sistema } \alpha \beta \text { e rotacionadas de }-\pi / 2 \\
& \mathrm{~s}_{\alpha+}(\mathrm{t}), \mathrm{s}_{\beta_{+}}(\mathrm{t}) \quad \text { parte real e parte imaginária dos sinais instantâneos de } \\
& \text { seqüência positiva com a defasagem corrigida } \\
& \mathrm{s}_{\alpha-}(\mathrm{t}), \mathrm{s}_{\beta}-(\mathrm{t}) \quad \text { parte real e parte imaginária dos sinais instantâneos de } \\
& \text { seqüência negativa com a defasagem corrigida } \\
& \omega \mathrm{t} \quad \text { ângulo variante no tempo que representa a posição angular do } \\
& \text { eixo girante }
\end{aligned}
$$$$
s_{r e f_{-} r-}(t), s_{r e f_{-} s-}(t), s_{r e f_{-} t-}(t) \text { sinais instantâneos de referência }
$$$$
\mathrm{p}, \mathrm{q} \quad \text { potências ativa e reativa instantâneas }
$$$$
\overline{\mathrm{p}}, \overline{\mathrm{q}} \quad \text { parte constante das potências ativa e reativa instantâneas }
$$$$
\widetilde{\mathrm{p}}, \widetilde{\mathrm{q}} \quad \text { parte oscilatória das potências ativa e reativa instantâneas }
$$$$
\mathrm{p}_{\mathrm{c}}, \mathrm{q}_{\mathrm{c}} \quad \text { potências relativas a pertubações }
$$$$
\mathrm{i}_{r}(\mathrm{t}), \mathrm{i}_{\mathrm{s}}(\mathrm{t}), \mathrm{i}_{\mathrm{t}}(\mathrm{t}) \quad \text { correntes instantâneas no sistema rst }
$$$$
\mathrm{v}_{\mathrm{r}}(\mathrm{t}), \mathrm{v}_{\mathrm{s}}(\mathrm{t}), \mathrm{v}_{\mathrm{t}}(\mathrm{t}) \quad \text { tensões instantâneas no sistema rst }
$$$$
\mathrm{i}_{\alpha}(\mathrm{t}), \mathrm{i}_{\beta}(\mathrm{t}) \quad \text { correntes instantâneas no sistema } \alpha \beta
$$$$
v_{\alpha}(t), v_{\beta}(t) \quad \text { tensões instantâneas no sistema } \alpha \beta
$$$$
\psi \quad \text { ângulo qualquer em radianos }
$$$$
\overrightarrow{\mathrm{d}}, \overrightarrow{\mathrm{q}} \quad \text { vetores do sistema dq }
$$$$
\mathrm{S}_{\mathrm{d}}(\mathrm{t}), \mathrm{S}_{\mathrm{q}}(\mathrm{t}) \quad \text { sinais instantâneos no sistema } \mathrm{dq}
$$$$
\tilde{s_{d}}(t), \tilde{s_{q}}(t) \quad \text { parcelas oscilatórias dos sinais instantâneos no sistema dq }
$$$$
\overline{s_{d}}(t), \overline{s_{q}}(t) \quad \text { parcelas médias dos sinais instantâneos no sistema dq }
$$$$
s_{d}^{\prime}(t), s_{q}^{\prime}(t) \quad \text { sinais instantâneos no sistema d'q' }
$$$$
\tilde{s_{d}^{\prime}}(t), \tilde{s_{q}^{\prime}}(t) \quad \text { parcelas oscilatórias dos sinais instantâneos no sistema d'q' }
$$$$
\overline{s_{d}^{\prime}}(t), \overline{s_{q}^{\prime}}(t) \quad \text { parcelas médias dos sinais instantâneos no sistema d'q' }
$$ 


$$
\begin{aligned}
& \tilde{s_{d n}}(t), \tilde{s_{q n}}(t) \quad \text { parcelas oscilatórias dos sinais instantâneos de seqüência } \\
& \text { negativa no sistema dq }
\end{aligned}
$$$$
\mathrm{s}_{\alpha \mathrm{c}}(\mathrm{t}), \mathrm{s}_{\beta c}(\mathrm{t}) \quad \text { parte real e parte imaginária dos sinais instantâneos de }
$$$$
\text { compensação }
$$$$
\mathrm{V}_{r_{-} P L L}, \mathrm{~V}_{\mathrm{S}_{-} P L L}, \mathrm{~V}_{\mathrm{t}} \mathrm{PLL} \quad \text { tensões instantâneas produzidas pelo PLL }
$$$$
\dot{S_{-}} \quad \text { fasor do sinal de seqüência negativa }
$$$$
\dot{S}_{+} \quad \text { fasor do sinal de seqüência positiva }
$$$$
\dot{S}_{0} \quad \text { fasor do sinal de seqüência nula }
$$$$
\dot{S}_{R}, \dot{S_{S}}, \dot{S_{T}} \quad \text { fasores dos sinais de linha }
$$

M matriz de conversão de valores no sistema rst para componentes simétricas

$S_{r e f_{-} r-}, S_{r e f_{-} s_{-}}, S_{r e f_{-} t-} \quad$ fasores dos sinais de referência obtidos pelo método de extração

$\mathrm{S}_{\mathrm{r} 0}(\mathrm{t}), \mathrm{S}_{\mathrm{s} 0}(\mathrm{t}), \mathrm{S}_{\mathrm{t} 0}(\mathrm{t}) \quad$ sinais instantâneos de sequência zero

$s_{r-}(t), s_{s-}(t), s_{t-}(t) \quad$ sinais instantâneos de seqüência negativa

$s_{r_{+}}(t), s_{s+}(t), s_{t+}(t) \quad$ sinais instantâneos de seqüência positiva

$\mathrm{s}_{\mathrm{r}-90}(\mathrm{t}), \mathrm{S}_{\mathrm{s}-90}(\mathrm{t}), \mathrm{s}_{\mathrm{t}-90}(\mathrm{t})$ sinais instantâneos atrasados de $\pi / 2$ no sistema rst número de amostras por ciclo da rede

$\mathrm{S}_{\mathrm{r} 60}(\mathrm{t}), \mathrm{S}_{\mathrm{s} 60}(\mathrm{t}), \mathrm{S}_{\mathrm{t} 60}(\mathrm{t})$ sinais instantâneos adiantados de $\pi / 3$ no sistema rst $\mathrm{S}_{\mathrm{r}-60}(\mathrm{t}), \mathrm{S}_{\mathrm{s}-60}(\mathrm{t}), \mathrm{S}_{\mathrm{t}-60}(\mathrm{t})$ sinais instantâneos atrasados de $\pi / 3$ no sistema rst $\mathrm{S}_{\mathrm{rh}}(\mathrm{t}), \mathrm{S}_{\mathrm{sh}}(\mathrm{t})$ e $\mathrm{S}_{\mathrm{th}}(\mathrm{t})$ sinais instantâneos com harmônicas $\mathrm{n} \quad$ número inteiro $(0,1,2, .$.
A B, C ou D operação de extração
Ibalanceada correntes de linha da rede balanceadas
Idesbalanceada correntes de linha da carga desbalancedas Icompensação correntes injetadas para compensação do desequilíbrio lcomp_ref correntes de referência para compensação do desequilíbrio $\mathrm{i}_{\mathrm{r}}(\mathrm{t}), \mathrm{i}_{\mathrm{s}}(\mathrm{t}), \mathrm{i}_{\mathrm{t}}(\mathrm{t}) \quad$ correntes instantâneas no sistema rst $i_{\text {ref_r- }}(t), i_{\text {ref_s- }}(t), i_{\text {ref_t }}{ }_{-}(t)$ correntes instantâneas de referência 
$\mathrm{i}_{\mathrm{r}-}(\mathrm{t}), \mathrm{i}_{\mathrm{s}-}(\mathrm{t}), \mathrm{i}_{\mathrm{t}-}(\mathrm{t}) \quad$ correntes instantâneas de seqüência negativa $\mathrm{i}_{\mathrm{rcc}}(\mathrm{t}), \mathrm{i}_{\mathrm{sc}}(\mathrm{t}), \mathrm{i}_{\mathrm{tc}}(\mathrm{t}) \quad$ correntes instantâneas da rede após a compensação $\mathrm{V}_{\mathrm{dm}}$ tensão instantânea do capacitor do barramento CC

$\mathrm{V}_{\mathrm{Lr},} \mathrm{V}_{\mathrm{Ls}}, \mathrm{V}_{\mathrm{Lt}} \quad$ queda de tensão instantânea no indutor de acoplamento

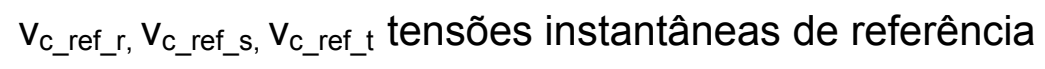

$i_{\_} d c_{-}, i_{\_} d c_{\_} s, i_{-} d c_{-} t$ correntes instantâneas de compensação para o regulador do barramento $\mathrm{CC}$

Verro erro instantâneo de tensão no barramento CC

$V_{\mathrm{d}_{\text {_ref }}} \quad$ tensão de referência do barramento $\mathrm{CC}$

$\mathrm{I}_{\text {ref_Pl }} \quad$ corrente instantânea de saída do controlador PI (malha de tensão)

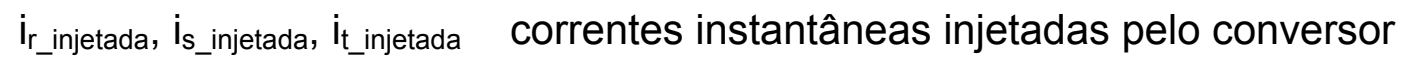

$\mathrm{X}$ grau de desequilíbrio de corrente

$\dot{I_{-}}$ fasor da corrente de seqüência negativa

$\dot{\mathrm{I}_{+}}$ fasor da corrente de seqüência positiva 


\title{
SUMÁRIO
}

\author{
LISTA DE TABELAS \\ LISTA DE FIGURAS \\ LISTA DE ABREVIATURAS E SIGLAS \\ LISTA DE SÍMBOLOS \\ RESUMO \\ ABSTRACT
}

1 INTRODUÇÃO

2 MÉTODOS DE EXTRAÇÃO DE SEQÜÊNCIA POSITIVA, 22 NEGATIVA E/OU HARMÔNICOS

2.1 Métodos baseados na teoria de vetores espaciais 23

2.1.1 Sistema de referência fixa 25

2.1.1.1 Método de deteç̧ão em tempo real através de vetores 25 espaciais (DTRVE)

2.1.2 Sistema de Referência girante 29

2.2 Método baseado na teoria da decomposição em seqüência 34 negativa, positiva e zero (Método Proposto)

3 MÉTODO PROPOSTO DE EXTRAÇÃO 
3.1 Demonstração matemática da operação $A$ aplicada a um sinal senoidal e periódico qualquer

3.2 Demonstração matemática da operação B aplicada a um sinal senoidal e periódico qualquer

3.3 Demonstração matemática das operações aplicadas a um sinal com conteúdo harmônico

3.3.1 Demonstração matemática da operação $A$ aplicada a um 45 sinal com conteúdo harmônico

3.3.2 Demonstração matemática da operação B aplicada a um 48 sinal com conteúdo harmônico

3.4 Estratégias para separar os distúrbios individualmente 52

4 SIMULAÇÃO NUMÉRICA E IMPLEMENTAÇÃO EM DSP DO 54 ALGORITMO DE EXTRAÇÃO PROPOSTO

4.1 Resposta em Regime 55

4.2 Resposta Transitória 65

4.3 Influências da variação da freqüência da rede 70

4.4 Tempos de execução 86

4.5 Observações gerais 86

5 APLICAÇÕES DO EXTRATOR 87

5.1 Exemplo de aplicação do extrator - simulação de um filtro ativo de potência

5.2 Exemplo de aplicação do extrator - simulação de um relé de seqüência negativa

6 CONCLUSÕES E SUGESTÕES PARA A CONTINUIDADE DO TRABALHO

LISTA DE REFERÊNCIAS

APÊNDICE A - MÉTODO PROPOSTO DE EXTRAÇÃO 103 APLICADO A UM SINAL COM SEQUÊNCIA ZERO

AP.1 Demonstração matemática da operação $A$ aplicada a um 104 sinal contendo sequência zero

AP.2 Demonstração matemática da operação B aplicada a um 104 sinal contendo sequência zero AP.3 Resposta em regime 
AP.4 Análise da influência da freqüência do sinal de seqüência zero

APÊNDICE B - IMPLEMENTAÇÃO DO BUFFER 


\section{INTRODUÇÃO}

Apresentam-se as conseqüências da operação do sistema elétrico contendo cargas desbalanceadas com correntes distorcidas e os objetivos desta tese.

Distorções e desequilíbrios nas correntes que circulam pela rede elétrica provocam diversos efeitos negativos na mesma. Equipamentos eletrônicos alimentados por retificadores a diodos e tiristores (aplicações em eletrodomésticos, drivers de motores, reatores eletrônicos para iluminação, fornos de indução etc.), por exemplo, têm comportamento não linear, drenando correntes distorcidas da rede elétrica, mesmo quando alimentados por tensão perfeitamente senoidal. Os efeitos negativos destes harmônicos de corrente são notados no sobreaquecimento de dispositivos elétricos (máquinas rotativas, transformadores e capacitores de correção de fator de potência) e distorções na forma senoidal da tensão de fornecimento, o que pode prejudicar o funcionamento de circuitos mais sensíveis. Tensões desequilibradas provocadas por cargas desbalanceadas causam efeitos indesejáveis como perdas adicionais em motores e geradores com evidente redução na vida útil desses equipamentos, atuação da proteção contra sobrecarga provocada pela circulação de correntes de seqüência negativa em motores de indução, aumento da ondulação ("ripple”) na tensão de saída dos retificadores e saturação de transformadores (IEEE 519-1992), (Penteado Jr., 1985), (Robba et al., 1996), (Watanabe; Aredes, 1998), (Senini; Wolfs, 2000) e (Senini; Wolfs, 2002).

A detecção em tempo real de desequilíbrios e harmônicos, de tensão ou de corrente é exigida em aplicações ligadas à compensação ativa de perturbações e relés de proteção. Uma breve descrição das estratégias mais conhecidas para a extração de harmônicos e/ou desequilíbrios é apresentada no capítulo 2.

Baseado no trabalho anteriormente desenvolvido por (Cutri, 2004), (Cutri; Matakas Jr., 2004), propõe-se (cap. 3) um algoritmo (Referência Instantânea de Filtragem Instantaneous Filter Reference - IFR) baseado na teoria de componentes simétricas que não requer nenhuma transformação de base nem filtragem do sinal e apenas utiliza 
cálculos algébricos. Esta nova abordagem permite obter apenas a seqüência positiva ou negativa da componente fundamental e/ou os harmônicos presentes no sinal. A validação experimental do algoritmo proposto é apresentada no capítulo 4.

Uma breve explanação sobre as possíveis aplicações do algoritmo proposto é apresentada no capítulo 5. Para ilustrar as possíveis aplicações, as simulações de um Filtro Ativo de Potência e de um Relé de Proteção são apresentadas respectivamente nas secções 5.1 e 5.2. Um diagrama de blocos explicitando a estrutura desta tese é apresentado na Fig.1.

\section{ESTRUTURA DA TESE - DIAGRAMA DE BLOCOS}

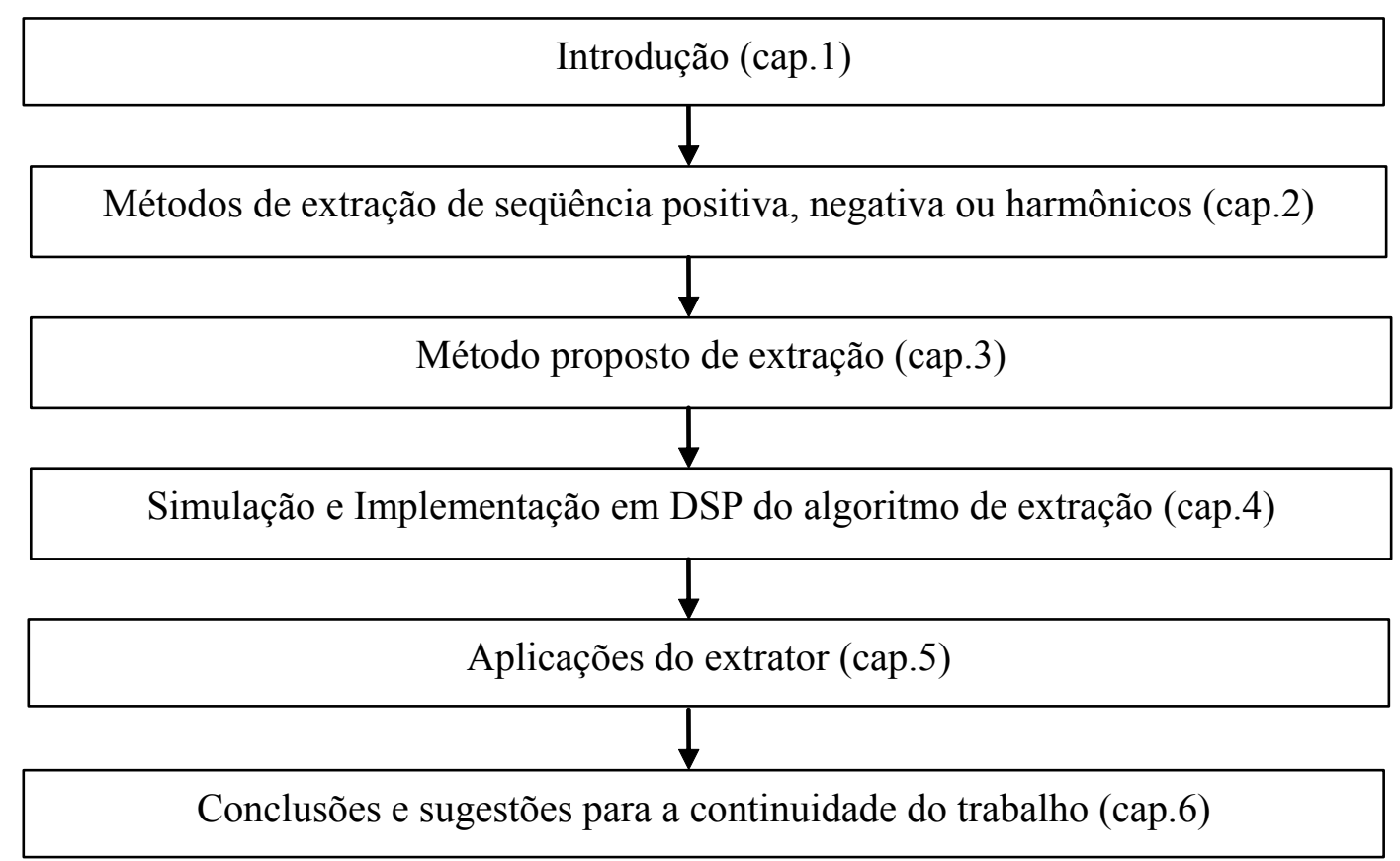

Fig.1 - Diagrama de Blocos - Estrutura da Tese 


\section{MÉTODOS DE EXTRAÇÃO DE SEQÜÊNCIA POSITIVA, NEGATIVA E/OU HARMÔNICOS}

Os métodos de extração de seqüência positiva, negativa e/ou harmônicos disponíveis na literatura são agrupados segundo suas características comuns e têm suas notações uniformizadas.

Muitas estratégias foram desenvolvidas para a extração de harmônicos e/ou desequilíbrios $^{1}$, destacando-se: referência síncrona positiva (RSP) e referência síncrona negativa (RSN) (Hafner et al., 1997), (Choi et al., 2000) e (Senini; Wolfs, 2000), a detecção através de vetores espaciais (DTRVE) (Zhang; Xu, 2001) e a injeção direta de seqüência negativa (DSNI) (Cutri; Matakas Jr., 2003).

Um estudo comparativo entres estas estratégias é apresentado em (Cutri, 2004).

Neste capítulo apresenta-se uma breve descrição das estratégias mais conhecidas agrupadas de acordo com a Fig.2-1.

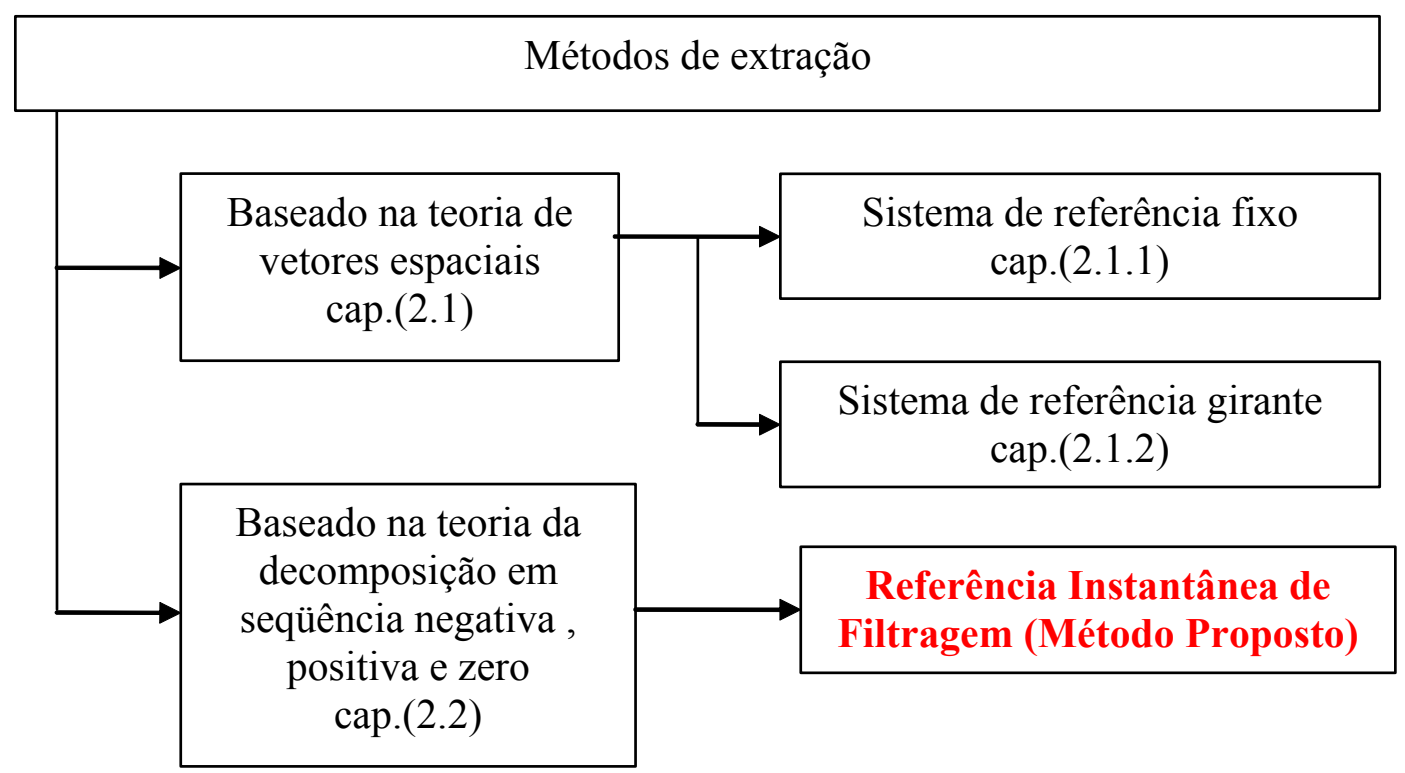

Fig.2-1 - Diagrama de Blocos - Métodos de extração das seqüências positiva, negativa e/ou harmônicos.

\footnotetext{
1 O Método das potências ativa e reativa instantâneas (PQ) (Akagi et al, 1984), (Akagi; Nabae, 1993) e (Watanabe; Aredes, 1998) não será incluído nesta seleção pois não permite a separação de harmônicos e sequências positiva e negativa.
} 


\subsection{Métodos baseados na teoria de vetores espaciais}

A Teoria de Vetores Espaciais é adequada para o tratamento matemático de valores instantâneos de tensões e correntes em circuitos trifásicos:

Segundo (Ferrero et al. ,1993), (Robba et al., 1996), (Matakas Jr. ,1998) e (Watanabe; Aredes, 1998), um terno de sinais instantâneos $\mathrm{s}_{\mathrm{r}}(\mathrm{t}), \mathrm{s}_{\mathrm{s}}(\mathrm{t}), \mathrm{s}_{\mathrm{t}}(\mathrm{t})$, de um sistema trifásico a três fios pode ser associado a um vetor espacial $\vec{S}$ no plano $\alpha \beta$ (Fig. 2.1-1), de acordo com a eq.(2.1-1). Mostra-se pela Fig. 2.1-1 e pela eq.(2.1-1) que o vetor $\vec{S}$ pode ser escrito tanto como uma combinação linear dos vetores $\overrightarrow{\mathrm{r}}, \overrightarrow{\mathrm{s}}, \overrightarrow{\mathrm{t}}$, como dos vetores $\vec{\alpha}, \vec{\beta}$. Os vetores $\overrightarrow{\mathrm{r}}, \overrightarrow{\mathrm{s}}, \overrightarrow{\mathrm{t}}$ são igualmente defasados de $120^{\circ}$ e não constituem uma base.

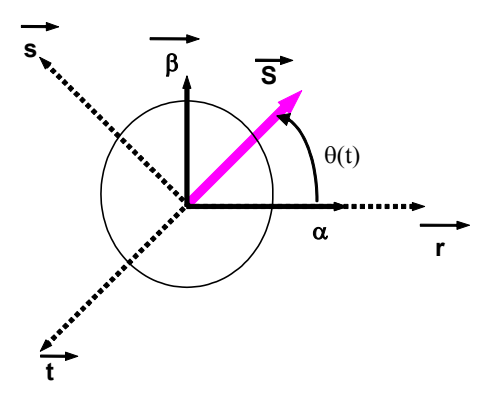

Fig. 2.1-1 - Vetor espacial $\vec{S}$, e os sistemas de coordenadas rst e $\alpha \beta$

$$
\vec{S}=\frac{2}{3} \cdot\left(s_{r}(t) \cdot \vec{r}+s_{s}(t) \cdot \vec{s}+s_{t}(t) \cdot \vec{t}\right)=s_{\alpha}(t) \cdot \vec{\alpha}+s_{\beta}(t) \cdot \vec{\beta}
$$

Neste desenvolvimento, não serão consideradas as componentes de seqüência zero dos sinais, sendo a soma dos sinais instantâneos dadas pela eq.(2.1-2).

$$
\mathrm{s}_{\mathrm{r}}(\mathrm{t})+\mathrm{s}_{\mathrm{s}}(\mathrm{t})+\mathrm{s}_{\mathrm{t}}(\mathrm{t})=0
$$

A transformação de coordenadas do sistema rst $\left(\mathrm{s}_{\mathrm{r}}(\mathrm{t}), \mathrm{s}_{\mathrm{s}}(\mathrm{t}), \mathrm{s}_{\mathrm{t}}(\mathrm{t})\right)$ para o sistema $\alpha \beta$ $\left(s_{\alpha}(t), s_{\beta}(t)\right)$, também conhecida como transformada de Clarke (Clarke ,1943), é uma transformação algébrica de um sistema trifásico em um sistema de referência 
estacionário onde se passa a trabalhar apenas com duas variáveis (sistema $\alpha \beta$ ), o que proporciona menor complexidade no tratamento das variáveis do sistema.

A relação entre os coeficientes $s_{\alpha}(t)$ e $s_{\beta}(t)$ e $s_{r}(t), s_{s}(t), s_{t}(t)$ dos sistemas $\alpha \beta$ e rst respectivamente é definida pela eq.(2.1-3).

$$
\left[\begin{array}{l}
s_{\alpha}(t) \\
s_{\beta}(t)
\end{array}\right]=\frac{2}{3}\left[\begin{array}{ccc}
1 & -1 / 2 & -1 / 2 \\
0 & \sqrt{3} / 2 & -\sqrt{3} / 2
\end{array}\right] \cdot\left[\begin{array}{l}
s_{r}(t) \\
s_{s}(t) \\
s_{t}(t)
\end{array}\right]
$$

Mostra-se que $s_{r}(t)+s_{0}(t), s_{s}(t)+s_{0}(t)$ e $s_{t}(t)+s_{0}(t)$ também representam o mesmo vetor $\vec{S}$. Por este motivo, a anti-transformada, ou seja, o retorno dos valores expressos no sistema $\alpha \beta$ para o sistema rst possui infinitas soluções. Para o caso particular da eq.(2.1-2), ou seja, seqüência zero nula, a antitransformada é dada pela eq.(2.1-4).

$$
\left[\begin{array}{l}
s_{r}(t) \\
s_{s}(t) \\
s_{t}(t)
\end{array}\right]=\left[\begin{array}{cc}
1 & 0 \\
-1 / 2 & \sqrt{3} / 2 \\
-1 / 2 & -\sqrt{3} / 2
\end{array}\right] \cdot\left[\begin{array}{l}
s_{\alpha}(t) \\
s_{\beta}(t)
\end{array}\right]
$$

O lugar geométrico do vetor $\vec{S}$ gerado por um conjunto de sinais trifásicos senoidais e equilibrados com valor de pico $S_{p}$ é um círculo com raio $S_{p}$. Adota-se que os sinais de seqüência positiva produzem um vetor $\vec{S}$ que gira no sentido antihorário e os sinais de seqüência negativa produzem um vetor no sentido oposto.

Além da notação vetorial, como apresentado, tem-se a notação complexa onde os eixos $\alpha$ e $\beta$ correspondem aos eixos real e imaginário do plano complexo. $O$ vetor $\vec{S}$ passa a ser representado pela eq.(2.1-5).

$$
\vec{S}=\frac{2}{3} \cdot\left(s_{r}(t) \cdot 1+s_{s}(t) \cdot e^{j \cdot \frac{2 \pi}{3}}+s_{t}(t) \cdot e^{-j \cdot \frac{2 \pi}{3}}\right)=s_{\alpha}(t)+j \cdot s_{\beta}(t)=S(t) \cdot e^{j \cdot \theta(t)}=S(t) \overline{\theta(t)}
$$




\subsubsection{Sistema de referência fixa}

\subsubsection{Método de detecção em tempo real através de vetores espaciais (DTRVE)}

Em (Zhang; Xu, 2001), para uma sinal genérico com harmônicos e desequilíbrios, associam-se os sinais $\mathrm{s}_{\mathrm{r}}(\mathrm{t}), \mathrm{s}_{\mathrm{s}}(\mathrm{t}), \mathrm{s}_{\mathrm{t}}(\mathrm{t})$ a um vetor espacial instantâneo $\vec{S}(t)$, em um sistema de referência fixo $(\alpha \beta)$, utilizando-se a eq.(2.1-5).

O vetor $\vec{S}(t)$ é descrito na notação complexa por $\vec{S}=S(t) \sqrt{\theta(t)}$, com $S(t)$ e $\theta(\mathrm{t})$ variantes no tempo, de modo que $\vec{S}(t)$ percorra um percurso arbitrário, conforme mostrado a seguir na Fig.2.1.1-1. Na notação complexa, o eixo $\alpha$ corresponde à parte real e o eixo $\beta$ à parte imaginária.

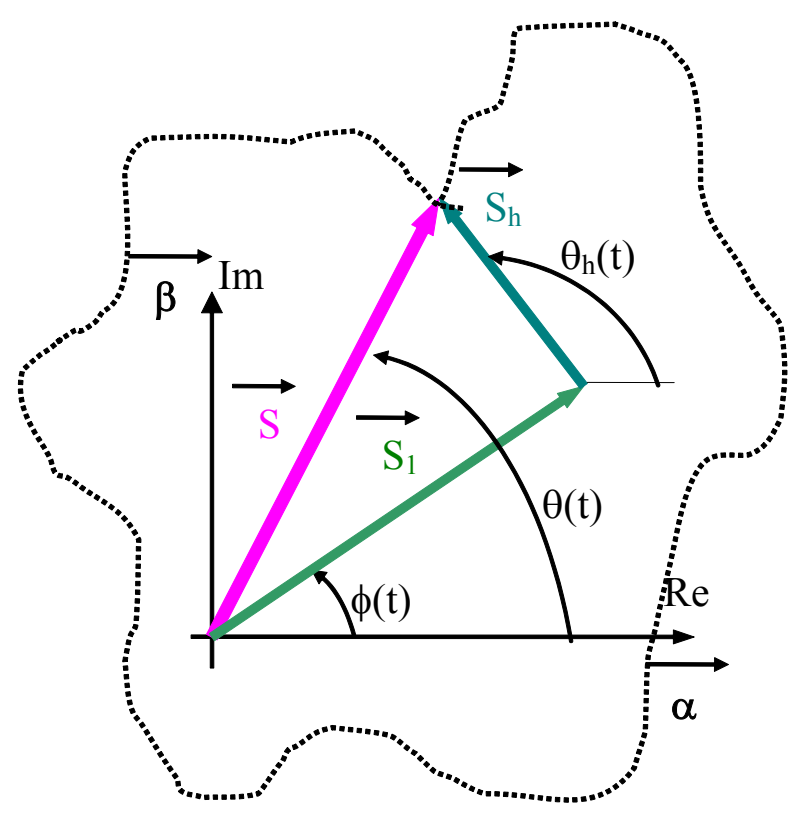

Fig. 2.1.1-1 - Vetor $\vec{S}$ e sua trajetória (pontilhada)

O vetor $\vec{S}$ pode ser decomposto em duas partes, $\vec{S}_{1}=S_{1}(t) \overline{\phi(t)}$, correspondente aos componentes do sinal na freqüência fundamental (Fig.2.1.1-1 em verde), e $\vec{S}_{h}=S_{h}(t) \overline{\phi_{h}(t)}$ que inclui todas as componentes harmônicas (Fig.2.1.1-1 em azul). 
O vetor $\vec{S}_{1}(t)$ pode ainda ser decomposto nas parcelas $\vec{S}_{+}(\mathrm{t})$ e $\overrightarrow{S_{-}}(\mathrm{t})$, correspondentes aos componentes de seqüência positiva e negativa na freqüência fundamental, de acordo com a eq.(2.1.1-1).

$$
\vec{S}_{1}(t)=\vec{S}_{+}(t)+\vec{S}_{-}(t)=S_{+}(t) \overline{\phi^{+}(t)}+S_{-}(t) \overline{\phi^{-}(t)}
$$

onde

$$
\begin{aligned}
& \phi^{+}(t)=\phi_{0}^{+}+\varpi . t \mathrm{e} \\
& \phi^{-}(t)=\phi_{0}^{-}-\varpi . t .
\end{aligned}
$$

O método de (Zhang; Xu, 2001) permite o cálculo de $\vec{S}_{+}$e $\vec{S}_{-}$, seguindo a seqüência abaixo descrita. Calcula-se inicialmente o vetor $\vec{S}$ a partir da eq.(2.1-5), decompondo-o nas projeções $s_{\alpha}(t)$ e $s_{\beta}(t)$ (Fig.2.1.1-2) conforme as eqs.(2.1.1-2) e (2.1.1-3).

$$
\begin{array}{r}
s_{\alpha}(t)=S(t) \cdot \cos (\theta(t))=S_{+}(t) \cdot \cos \left(\phi^{+}(t)\right)+S_{-}(t) \cdot \cos \left(\phi^{-}(t)\right)+\sum_{2}^{\infty} S_{h}(t) \cdot \cos \left(\phi^{h}(t)\right) \\
(2 \cdot 1 \cdot 1-2) \\
s_{\beta}(t)=S(t) \cdot \operatorname{sen}(\theta(t))=S_{+}(t) \cdot \operatorname{sen}\left(\phi^{+}(t)\right)+S_{-}(t) \cdot \operatorname{sen}\left(\phi^{-}(t)\right)+\sum_{2}^{\infty} S_{h}(t) \cdot \operatorname{sen}\left(\phi^{h}(t)\right)
\end{array}
$$

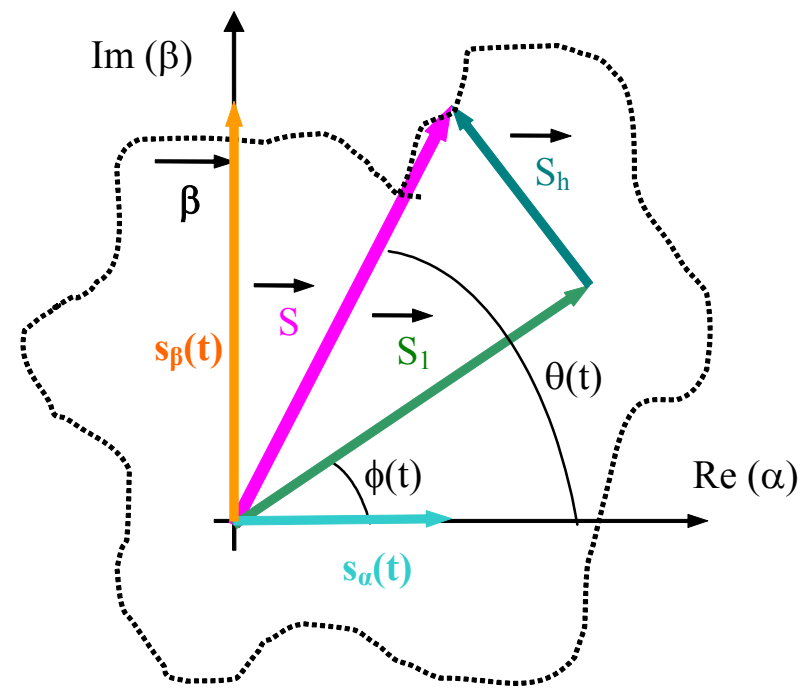

Fig. 2.1.1-2 - Projeção do Vetor $\vec{S}$ no sistema $\alpha \beta$ 
As parcelas $s_{\alpha}(t)$ e $s_{\beta}(t)$ são filtradas por dois filtros passa-baixas independentes (com ganho unitário (de modo a não alterar a amplitude dos sinais), e fase $(-\varphi)$ na freqüência fundamental) eliminando-se as componentes harmônicas e introduzindo uma defasagem $(-\varphi)$ na componente do sinal de freqüência fundamental conforme a Fig.2.1.1-3. Após a filtragem, os componentes $s_{\text {aff }}(t)$ (parte real) e $s_{p f}(t)$ (parte imaginária) que contêm as componentes das seqüências positiva $\left(s_{\alpha f+}(t) ; s_{\beta f+}(t)\right)$ e negativa $\left(s_{\alpha f-}(t) ; s_{\beta f-}(t)\right)$ da freqüência fundamental podem ser reescritas nas eqs.(2.1.1-4) e (2.1.1-5) respectivamente.

$$
\begin{aligned}
& s_{\alpha f}(t)=S_{+} \cdot \cos \left(\phi^{+}(t)-\varphi\right)+S_{-} \cdot \cos \left(\phi^{-}(t)-\varphi\right)=s_{a f+}(t)+s_{\alpha f-}(t) \\
& s_{\beta f}(t)=S_{+} \cdot \operatorname{sen}\left(\phi^{+}(t)-\varphi\right)-S_{-} \cdot \operatorname{sen}\left(\phi^{-}(t)-\varphi\right)=s_{\beta f+}(t)+s_{\beta f-}(t)
\end{aligned}
$$

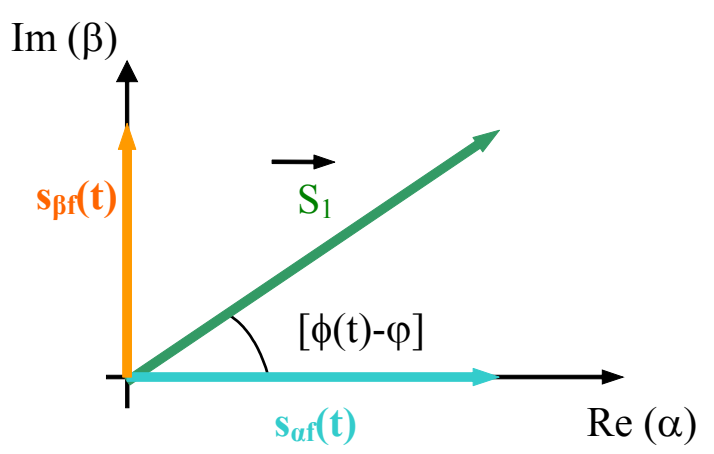

Fig. 2.1.1-3 - Projeção no sistema $\alpha \beta$ do Vetor $\overrightarrow{S_{1 f}}$ após a filtragem

Para separar as projeções das componentes de seqüência positiva $\left(\mathbf{s}_{\alpha f+}(t) ; \mathbf{s}_{\beta f+}(t)\right)$ e negativa $\left(s_{\alpha f-}(t) ; s_{\beta f-}(t)\right)$ de $s_{\alpha f}(t)$ e $s_{\beta f}(t)$, (Zhang; $\left.X u, 2001\right)$ propõe criar um novo vetor $\left(s_{\alpha f 90}(t) ; s_{\beta f} 90(t)\right)$, obtido a partir do vetor original $\left(s_{\alpha f}(t) ; s_{\beta f}(t)\right)$, rotacionando-o de $-\pi / 2$, o que resulta nas eqs.(2.1.1-6) e (2.1.1-7) respectivamente.

$$
\begin{aligned}
& S_{a f 90}(t)=S_{+} \cdot \cos \left(\phi^{+}(t)-\varphi-\frac{\pi}{2}\right)+S_{-} \cdot \cos \left(\phi^{-}(t)-\varphi-\frac{\pi}{2}\right)= \\
& S_{+} \cdot \operatorname{sen}\left(\phi^{+}(t)-\varphi\right)+S_{-} \cdot \operatorname{sen}\left(\phi^{-}(t)-\varphi\right) \\
& S_{\beta f 90}(t)=S_{+} \cdot \operatorname{sen}\left(\phi^{+}(t)-\varphi-\frac{\pi}{2}\right)-S_{-} \cdot \operatorname{sen}\left(\phi^{-}(t)-\varphi-\frac{\pi}{2}\right)= \\
& -S_{+} \cdot \cos \left(\phi^{+}(t)-\varphi\right)+S_{-} \cdot \cos \left(\phi^{-}(t)-\varphi\right)
\end{aligned}
$$


Os componentes desejados de seqüência positiva $\left(\mathrm{s}_{\alpha f+}(\mathrm{t}) ; \mathrm{s}_{\beta f+}(\mathrm{t})\right)$ e negativa $\left(\mathrm{s}_{\alpha \mathrm{f}-}(\mathrm{t})\right.$; $\left.\mathrm{s}_{\beta f}(\mathrm{t})\right)$ são obtidos a partir dos valores de $\left(\mathrm{s}_{\alpha f}(\mathrm{t}) ; \mathrm{s}_{\beta \mathrm{f}}(\mathrm{t}) ; \mathrm{s}_{\alpha f 90}(\mathrm{t}) ; \mathrm{s}_{\beta \mathrm{f} 90}(\mathrm{t})\right)$ utilizando-se as eqs.(2.1.1-8), (2.1.1-9), (2.1.1-10) e (2.1.1-11).

$$
\begin{array}{cc}
s_{\alpha f+}(t)=\frac{\left(s_{\alpha f}(t)-s_{\beta f 90}(t)\right)}{2}(2.1 .1-8) & s_{\alpha f-}(t)=\frac{\left(s_{\alpha f}(t)+s_{\beta f 90}(t)\right)}{2} \\
s_{\beta f+}(t)=\frac{\left(s_{\beta f}(t)+s_{\alpha f 90}(t)\right)}{2}(2.1 .1-10) & s_{\beta f-}(t)=\frac{\left(s_{\beta f}(t)-s_{\alpha f 90}(t)\right)}{2}
\end{array}
$$

Podem-se agrupar as eqs.(2.1.1-8), (2.1.1-9), (2.1.1-10) e (2.1.1-11) na matriz dada pela eq.(2.1.1-12).

$$
\cdot\left[\begin{array}{l}
s_{\alpha f+}(t) \\
s_{\beta f+}(t) \\
s_{\alpha f-}(t) \\
s_{\beta f-}(t)
\end{array}\right]=\frac{1}{2} \cdot\left[\begin{array}{cccc}
0 & -1 & 1 & 0 \\
1 & 0 & 0 & 1 \\
0 & 1 & 1 & 0 \\
-1 & 0 & 0 & 1
\end{array}\right] \cdot\left[\begin{array}{l}
s_{\alpha f 90}(t) \\
s_{\beta f 90}(t) \\
s_{\alpha f}(t) \\
s_{\beta f}(t)
\end{array}\right]
$$

Para se corrigir o deslocamento da fase causado pelo filtro $(-\varphi)$, é necessário rotacionar-se os vetores $\left(\mathrm{s}_{\alpha f+}(\mathrm{t}) ; \mathrm{s}_{\beta f+}(\mathrm{t})\right)$ e $\left(\mathrm{s}_{\alpha \mathrm{ff}-}(\mathrm{t}) ; \mathrm{s}_{\beta f}(\mathrm{t})\right)$ de acordo com $\mathrm{o}$ deslocamento $(\varphi)$, obtendo-se assim os valores dos componentes de seqüência positiva e negativa $\left(\mathrm{s}_{\alpha+}(\mathrm{t}) ; \mathrm{s}_{\beta+}(\mathrm{t}) ; \mathrm{s}_{\alpha-}(\mathrm{t}) ; \mathrm{s}_{\beta-}(\mathrm{t})\right)$ respectivamente nas eqs.(2.1.1-13) e $(2.1 .1-14)$.

$$
\begin{gathered}
{\left[\begin{array}{l}
s_{\alpha+}(t) \\
s_{\beta+}(t)
\end{array}\right]=\left[\begin{array}{cc}
\cos \varphi & -\sin \varphi \\
\sin \varphi & \cos \varphi
\end{array}\right] \cdot\left[\begin{array}{l}
s_{\alpha f+}(t) \\
s_{\beta f+}(t)
\end{array}\right]} \\
{\left[\begin{array}{l}
s_{\alpha-}(t) \\
s_{\beta-}(t)
\end{array}\right]=\left[\begin{array}{cc}
\cos \varphi & \sin \varphi \\
-\sin \varphi & \cos \varphi
\end{array}\right]\left[\begin{array}{l}
s_{\alpha f-}(t) \\
s_{\beta f-}(t)
\end{array}\right]}
\end{gathered}
$$

Os sinais instantâneos de seqüência positiva e/ou negativa $\left(s_{r e f_{-} r_{-}}(t), s_{r e f_{-} s^{-}}(t), s_{r e f_{-} t}(t)\right)$ são obtidos pela eq.(2.1-4), substituindo-se os valores de $s_{\alpha}(t)$ por $s_{\alpha+}(t)\left(s_{\alpha-}(t)\right)$ e $s_{\beta}(t)$ por $s_{\beta+}(t)\left(s_{\beta-}(t)\right)$.

O diagrama de blocos do Método DTRVE mostrando a extração da seqüência negativa é apresentado na Fig. 2.1.1-4. 


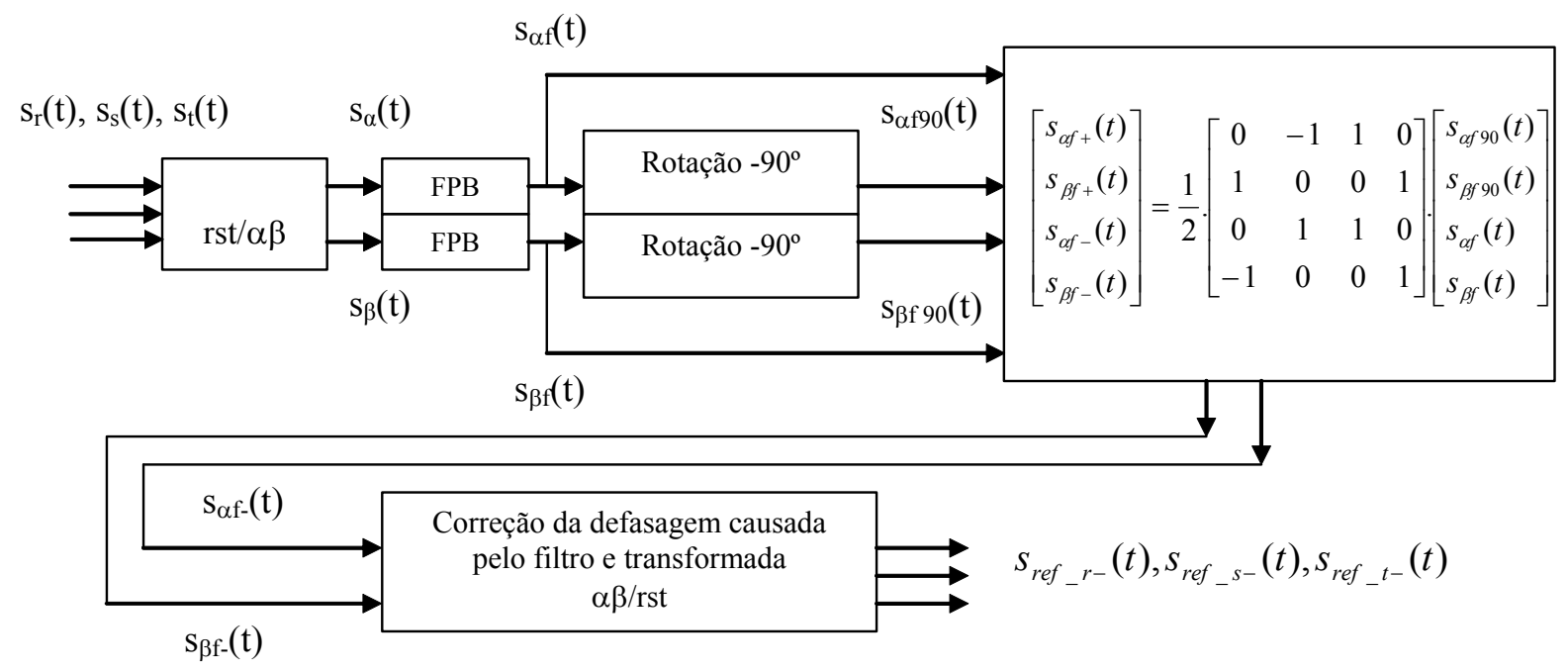

Fig. 2.1.1-4 - Diagrama de Blocos do Método DTRVE (extração seqüência negativa)

\subsubsection{Sistema de referência girante}

O método de referência girante positiva requer a mudança do sistema de coordenadas fixo $\alpha \beta$ (Seção 2.1) para o sistema de coordenadas girante dq. $O$ sistema dq gira com velocidade $\omega$ de modo que o ângulo ${ }^{2}$ entre os eixos $\alpha$ e $d(\beta$ e q) tenha valor ( $\omega \mathrm{t})$. A transformação das coordenadas do vetor do sinal no sistema $\alpha \beta$ (sistema de referência fixo) para o sistema dq (sistema girante), também chamada Transformada de Park (Hafner et al., 1997), (Choi et al., 2000) e (Senini; Wolfs, 2000) é efetuada pelas eqs.(2.1-3) e (2.1.2-1) e observada na Fig. 2.1.2-1.

$$
\left[\begin{array}{l}
s_{d}(t) \\
s_{q}(t)
\end{array}\right]=\left[\begin{array}{cc}
\cos (\omega t) & \operatorname{sen}(\omega t) \\
-\operatorname{sen}(\omega t) & \cos (\omega t)
\end{array}\right] \cdot\left[\begin{array}{l}
s_{\alpha}(t) \\
s_{\beta}(t)
\end{array}\right]
$$

\footnotetext{
${ }^{2}$ Para simplificar a explanação considerou-se o ângulo entre os eixos $\alpha$ e d com valor " $\omega$ t ". Para uma abordagem mais geral deve-se considerá-lo como sendo " $\omega t+\psi$ ".
} 


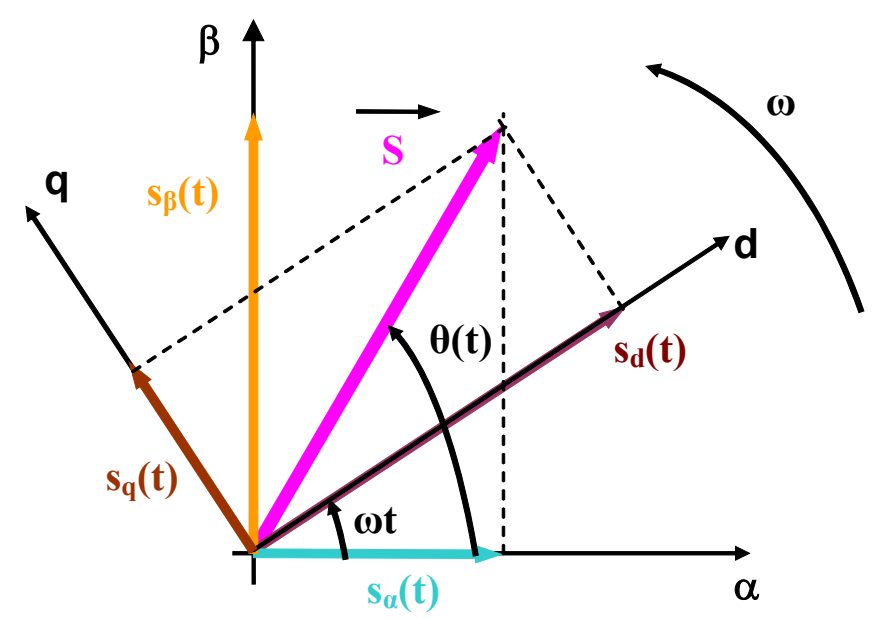

Fig. 2.1.2-1 - Vetores em um sistema girante de referência

Esse sistema está sendo rotacionado a uma velocidade constante em sincronismo com uma referência o que torna necessário um circuito de sincronismo do tipo PLL para a obtenção de $(\omega t)$.

O vetor $\vec{S}$ pode ser descrito pela soma das componentes fundamental $\vec{S}_{1}$ e harmônicos $\vec{S}_{h}$ mostrado na Fig. 2.1.1-1. O vetor da componente fundamental do sinal analisado $\vec{S}_{1}$ pode ser descrito pela soma dos vetores $\vec{S}_{+}$e $\vec{S}_{-}$correspondentes aos componentes de seqüência positiva e negativa da fundamental do sinal. No sistema $\alpha \beta, \overrightarrow{S_{+}}$tem velocidade angular $\omega$, enquanto $\overrightarrow{S_{-}}$ tem velocidade angular - $\omega$ conforme a Fig.2.1.2-2.

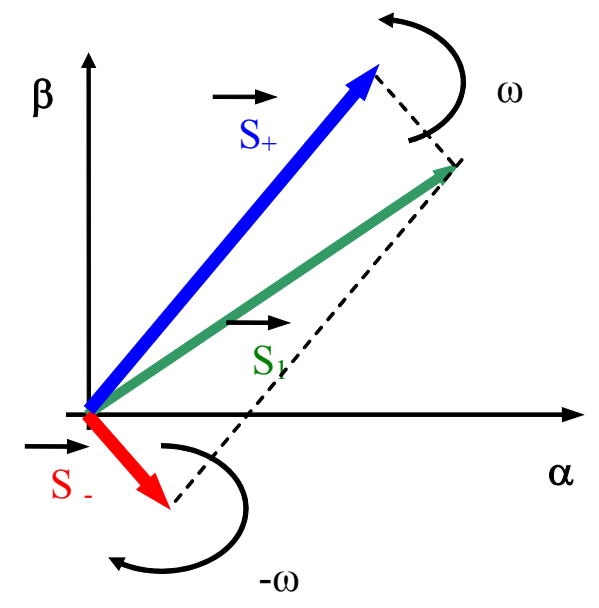

Fig. 2.1.2-2 - Vetores dos componentes fundamentais em um sistema fixo de referência 
No sistema de referência girante o vetor $\vec{S}_{+}$de seqüência positiva permanece parado. Deste modo, as projeções de $\overrightarrow{S_{+}}$nos eixo dq apresentam amplitude

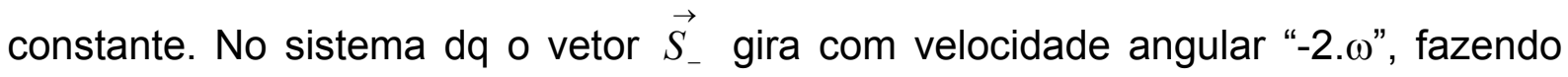

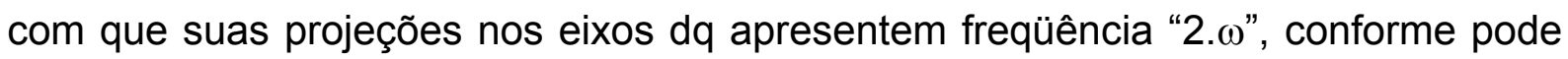
ser visto na Fig.2.1.2-3. Harmônicos de seqüência positiva de ordem $\mathrm{h}$ produzirão componentes no sistema dq com freqüência $(h-1) . \omega$, enquanto que os de seqüência negativa terão freqüência $(h+1) . \omega$. Assim, a parte oscilatória das coordenadas do vetor $\vec{S}$ no sistema dq é formada tanto por harmônicos como por componentes de seqüência negativa.

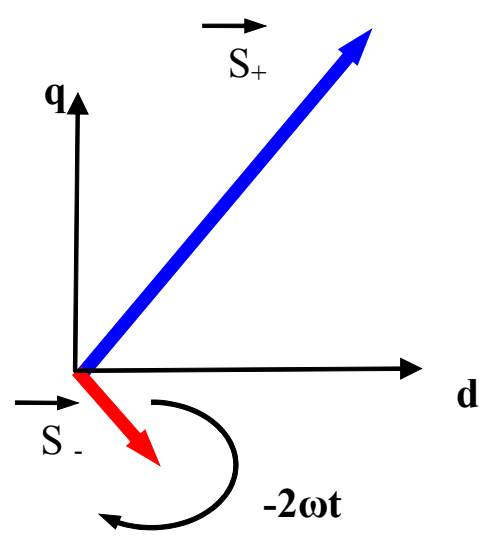

Fig. 2.1.2-3 - Vetores da componente fundamental representada em um sistema fixo e num sistema girante de referência

Portanto, no sistema dq o componente fundamental de seqüência positiva pode ser filtrado utilizando-se um filtro passa-baixa. A parte oscilatória $\left(\tilde{s_{d}}(t), \tilde{s_{q}}(t)\right)$ corresponde aos harmônicos e seqüência negativa extraídos do sinal no sistema dq. A proximidade entre as freqüências da parcela associada à seqüência negativa (2. $\omega$ ) e das parcelas associadas aos harmônicos $((h \pm 1) . \omega)$ nos sinais $\mathrm{s}_{d}$ e $\mathrm{s}_{\mathrm{q}}$, torna difícil sua separação pela filtragem. O diagrama de blocos do Método RSP mostrando a extração da seqüência negativa e harmônicos é apresentado na Fig. 2.1.2-4. 


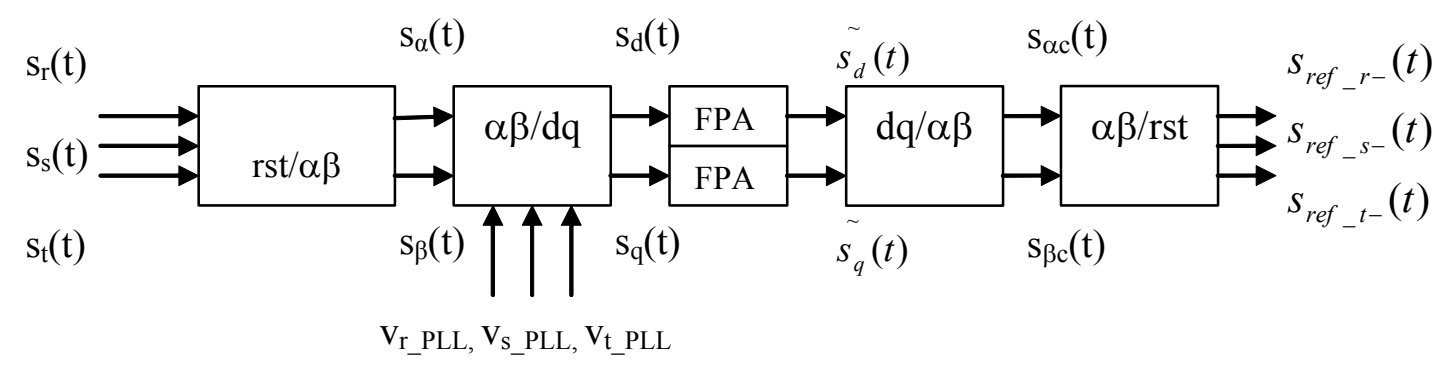

Fig. 2.1.2-4 - Diagrama de Blocos do Método RSP

(Hafner et al., 1997), (Choi et al., 2000) e (Senini; Wolfs, 2000) propõem utilizar uma estratégia semelhante à da referência girante positiva, para a extração da seqüência positiva de sinais distorcidas num método chamado de referência girante negativa (RSN). Neste método, após a extração do componente constante correspondente à seqüência positiva $\left(\overline{s_{d}}(t), \overline{s_{q}}(t)\right)$, representa-se o vetor associado às variáveis oscilantes $\left(\tilde{s_{d}}(t), \tilde{s_{q}}(t)\right)$ em um novo sistema d'q', que gira no sentido horário, com velocidade $-2 \omega$ com relação ao sistema dq original, utilizando a eq.(2.1.2-2).

$$
\left[\begin{array}{l}
s_{d}^{\prime}(t) \\
s_{q}^{\prime}(t)
\end{array}\right]=\left[\begin{array}{cc}
\cos (-2 \omega t) & \operatorname{sen}(-2 \omega t) \\
-\operatorname{sen}(-2 \omega t) & \cos (-2 \omega t)
\end{array}\right] \cdot\left[\begin{array}{c}
\tilde{s}_{d}(t) \\
-\tilde{s}_{q}(t)
\end{array}\right]
$$

No sistema d'q', a componente de seqüência negativa da fundamental apresentase como um valor constante $\left(\overline{s_{d}^{\prime}}(t), \overline{s_{q}^{\prime}}(t)\right)$ que pode ser isolado via um filtro passabaixa. Os sinais no sistema rst $\left(s_{\text {ref }{ }_{-} r}(t), s_{\text {ref_s }}(t), s_{\text {ref }} t(t)\right)$ são obtidos através da eq.(2.1.2-2), (2.1.2-3) e (2.1-4) substituindo-se os valores de $\tilde{s_{d}}(t)$ por $\tilde{s_{d n}}(t), \tilde{s_{q}}(t)$ por $\tilde{s_{q n}}(t), \mathbf{s}_{\alpha}(\mathrm{t})$ por $\mathbf{s}_{\alpha c}(\mathrm{t})$ e $\mathrm{s}_{\beta}(\mathrm{t})$ por $\mathrm{s}_{\beta c}(\mathrm{t})$.

$$
\left[\begin{array}{c}
\tilde{s_{d n}} \\
\tilde{s_{q n}}(t)
\end{array}\right]=\left[\begin{array}{cc}
\cos (-2 \omega t) & -\operatorname{sen}(-2 \omega t) \\
\operatorname{sen}(-2 \omega t) & \cos (-2 \omega t)
\end{array}\right] \cdot\left[\begin{array}{c}
\overline{s_{d}^{\prime}}(t) \\
\overline{s_{q}^{\prime}}(t)
\end{array}\right]
$$




$$
\left[\begin{array}{c}
s_{\alpha c}(t) \\
s_{\beta c}(t)
\end{array}\right]=\left[\begin{array}{cc}
\cos (\omega t) & -\operatorname{sen}(\omega t) \\
\operatorname{sen}(\omega t) & \cos (\omega t)
\end{array}\right] \cdot\left[\begin{array}{c}
\tilde{s}_{d}(t) \\
\tilde{s_{q}}(t)
\end{array}\right]
$$

O diagrama de blocos do Método RNP mostrando a extração da seqüência negativa é apresentado na Fig. 2.1.2-5.

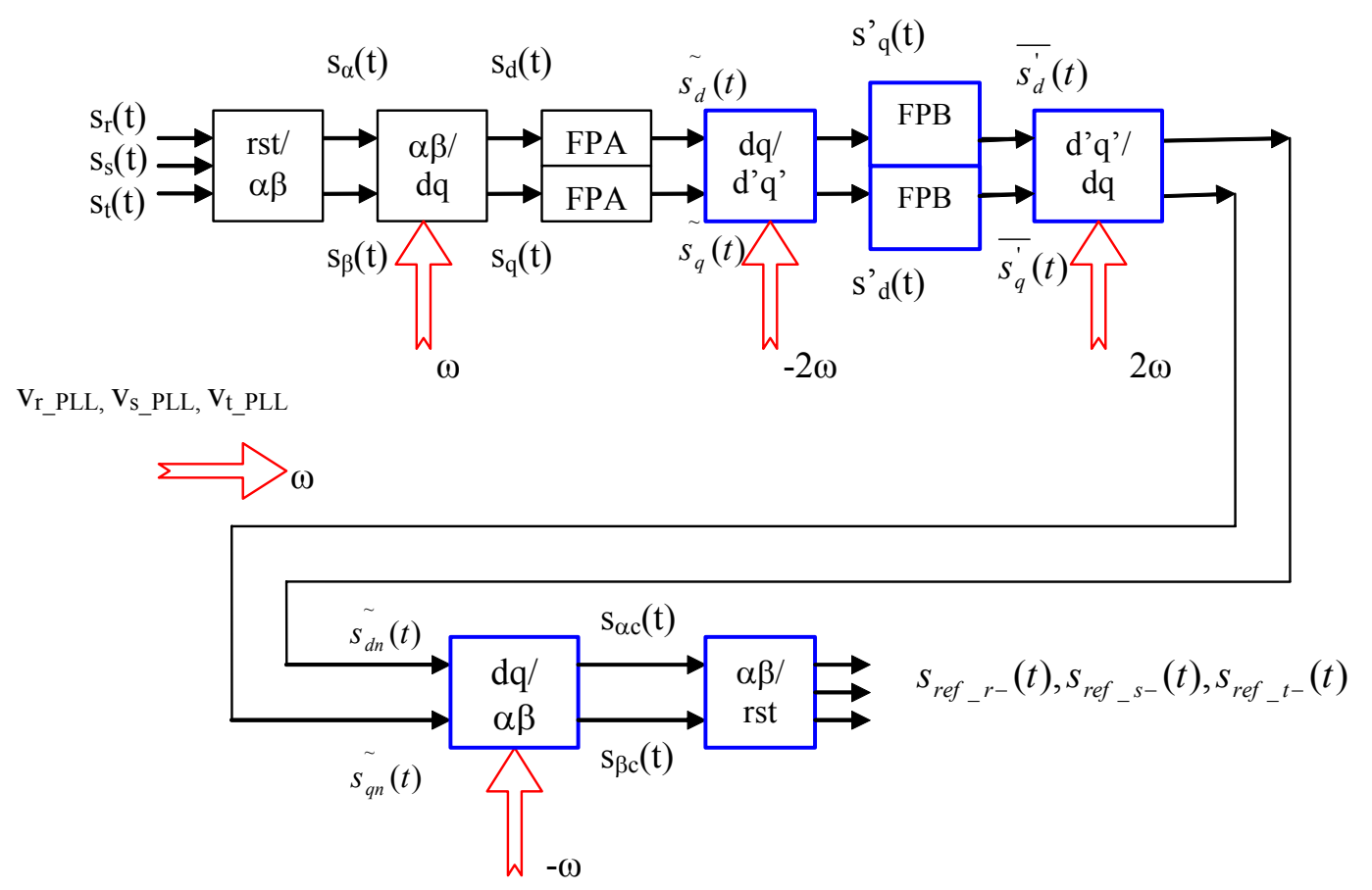

Fig. 2.1.2-5 - Diagrama de Blocos do Método RNP

Uma variante do método de Referência Girante Positiva chamada de Referência Girante Positiva Modificada é apresentada em (Marques, 1998). Nela, o ângulo de referência para rotação do sistema é calculado utilizando diretamente os sinais no sistema rst. Não necessitando assim de um circuito de sincronismo. A velocidade da referência girante pode não ser mais constante. Ela varia instantaneamente dependendo da forma de onda do sistema de tensão trifásico.

O ângulo de referência ( $\omega t$ ) é variável no tempo segundo as eqs.(2.1.2-3), (2.1.24) e (2.1.2-5).

$$
\begin{gathered}
{\left[\begin{array}{l}
s_{\alpha}(t) \\
s_{\beta}(t)
\end{array}\right]=\frac{2}{3} \cdot\left[\begin{array}{ccc}
1 & -1 / 2 & -1 / 2 \\
0 & \sqrt{3} / 2 & -\sqrt{3} / 2
\end{array}\right]\left[\begin{array}{l}
s_{r}(t) \\
s_{s}(t) \\
s_{t}(t)
\end{array}\right]} \\
\cos (\omega t)=\frac{s_{\alpha}(t)}{\sqrt{s_{\alpha}^{2}(t)+s_{\beta}^{2}(t)}}(2.1 .2-4) \quad \sin (\omega t)=\frac{s_{\beta}(t)}{\sqrt{s_{\alpha}^{2}(t)+s_{\beta}^{2}(t)}}
\end{gathered}
$$


(Marques, 1998) mostra que o método Referência Girante Positiva Modificada apresenta um desempenho idêntico ao método de Referência Girante Positiva se os sinais forem equilibrados e simétricos, no entanto o desempenho piora quando for distorcido ou desbalanceado, o que remete a necessidade de um circuito PLL.

\subsection{Método baseado na teoria da decomposição em seqüência negativa, positiva e zero (Método Proposto)}

A utilização deste método para a extração dos sinais instantâneos de seqüência positiva, negativa e harmônicos foi proposta inicialmente em (Cutri; Matakas Jr., 2003) baseando-se na teoria de componentes simétricos (Fortescue, 1918) e (Robba et al., 1996), no entanto a mesma não permitia a extração somente de desequilíbrios ou harmônicos separadamente. Nesta tese é proposto um aperfeiçoamento do método apresentado em (Deckmann, S.M.; Marafao, F.P., 2000), (Cutri; Matakas Jr., 2003) permitindo a extração somente das componentes de seqüência positiva, negativa e/ou harmônicos da fundamental. A extração é feita sem nenhuma transformação de sistema de coordenadas, operando-se diretamente com os sinais de linha medidos.

O diagrama de blocos do Método proposto é apresentado na Fig. 2.2-1.

Este método é descrito em detalhe no cap.3.

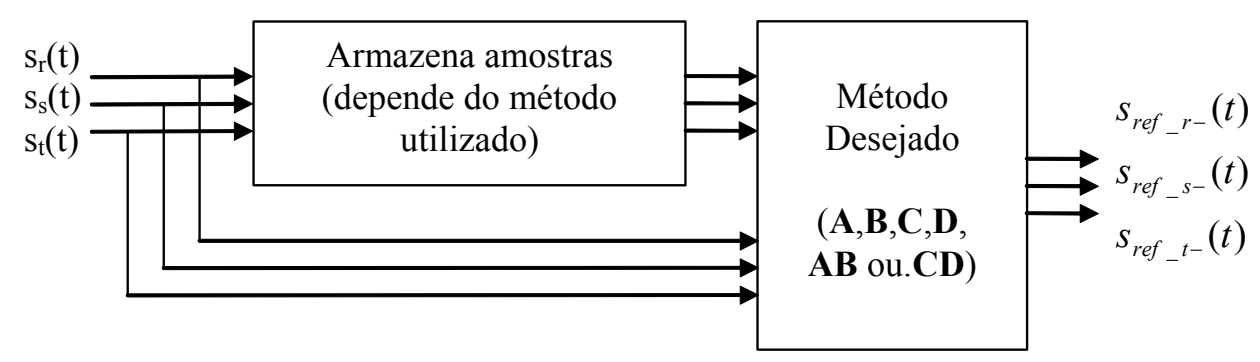

Fig. 2.2-1 - Diagrama de Blocos do Método Proposto 


\section{MÉTODO PROPOSTO DE EXTRAÇÃO}

O método de extração proposto nesta tese é apresentado.

A eq.(3.1) calcula os fasores das componentes simétricas $\left(\dot{S}_{0}, \dot{S}_{+}, \dot{S}_{-}\right)$associadas a um terno de grandezas senoidais de fase (correntes ou tensões) $\left(\dot{S}_{R}, \dot{S_{S}}, \dot{S_{T}}\right)$ (Fortescue, 1918) e (Robba et al., 1996).

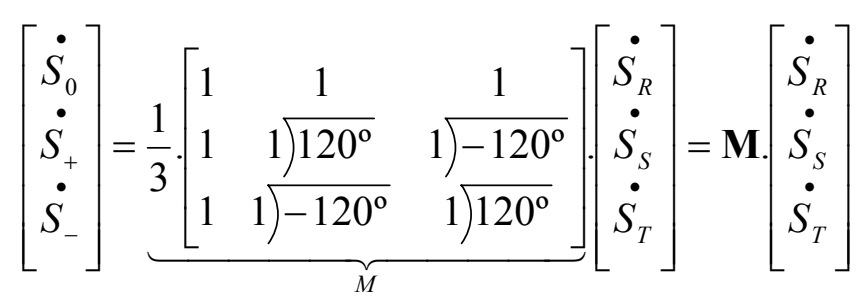

Para se extraírem as componentes que causam desequilíbrio basta que se separem os fasores de seqüência negativa e zero. Os fasores de seqüência zero podem ser obtidos pela eq. (3.2) enquanto que os fasores de seqüência negativa podem ser obtidos pela eq.(3.3).

$$
\left[\begin{array}{c}
\dot{S_{0}} \\
0 \\
0
\end{array}\right]=\frac{1}{3} \cdot\left[\begin{array}{lll}
1 & 1 & 1 \\
0 & 0 & 0 \\
0 & 0 & 0
\end{array}\right] \cdot\left[\begin{array}{c}
\dot{S}_{R} \\
\dot{S_{S}} \\
\dot{S_{T}}
\end{array}\right] \quad(3.2) \quad\left[\begin{array}{c}
0 \\
0 \\
\dot{S_{-}}
\end{array}\right]=\frac{1}{3} \cdot\left[\begin{array}{ccc}
0 & 0 & 0 \\
0 & 0 & 0 \\
1 & 1)-120^{\circ} & 1 \\
120^{\circ}
\end{array}\right] \cdot\left[\begin{array}{l}
\dot{S}_{R} \\
\dot{S}_{S} \\
\dot{S_{T}}
\end{array}\right]
$$

Para se obterem os fasores (seqüência negativa) extraídos na base rst basta multiplicar os fasores obtidos pela eq.(3.3) pela matriz inversa ( $\left.\mathbf{M}^{-1}\right)$ da eq.(3.1), obtendo a eq.(3.4).

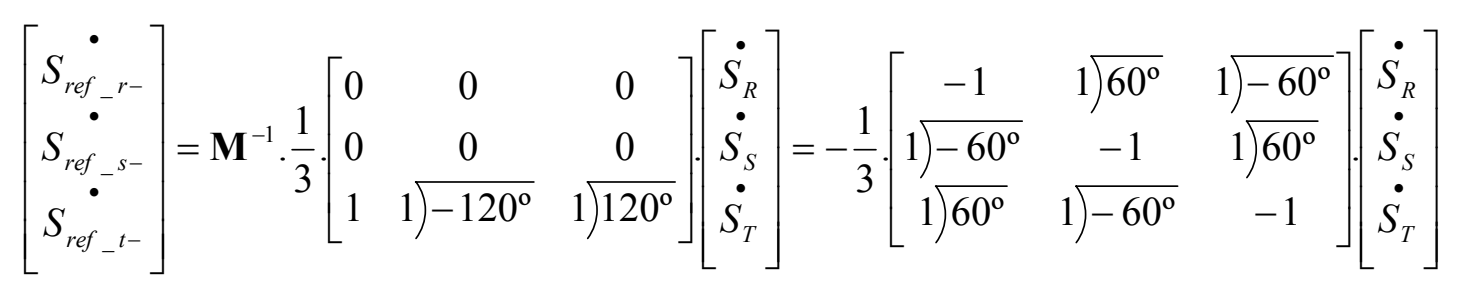

A matriz complexa da eq.(3.4) pode ser reescrita em duas sub-matrizes: uma contendo os termos reais e outra contendo os termos imaginários multiplicados por -j conforme a eq.(3.5). 


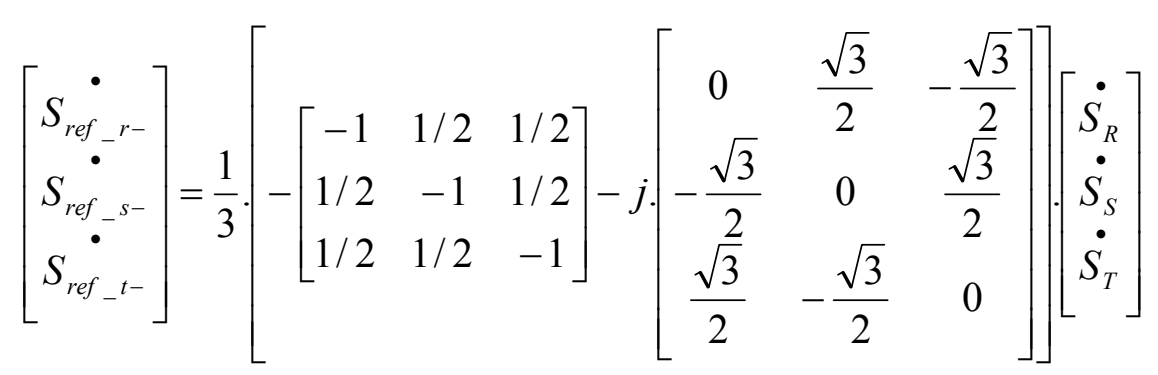

Para operação em regime permanente senoidal (Deckmann, S.M.; Marafao, F.P., 2000), (Cutri; Matakas Jr., 2003), (Cutri, 2004), (Cutri; Matakas Jr., 2004), (Cutri; Matakas Jr., 2007) as equações (3.4) e (3.5) podem ser reescritas no domínio do tempo, resultando nas equações (3.6) e (3.7). O deslocamento de fase de $60^{\circ}\left(-60^{\circ}\right)$ ou $-90^{\circ}\left(90^{\circ}\right)$ pode ser implementado pelo atraso no tempo do sinal original por um intervalo de tempo correspondente ao ângulo desejado na freqüência fundamental. $\mathrm{O}$ atraso pode ser implementado armazenando-se um determinado número de amostras medidas. O atraso de $90^{\circ}$ (sinais $\left(\mathrm{s}_{\mathrm{r}-90}(\mathrm{t}), \mathrm{s}_{\mathrm{s}-90}(\mathrm{t})\right.$ e $\mathrm{s}_{\mathrm{t}-90}(\mathrm{t})$ ) pode ser implementado armazenando-se as últimas N/4 amostras medidas. O avanço de $90^{\circ}$ pode ser obtido através da multiplicação dos sinais atrasados de $90^{\circ}$ por -1 . $O$ atraso de $60^{\circ}\left(\mathrm{s}_{\mathrm{r}-60}(\mathrm{t}), \mathrm{s}_{\mathrm{s}-60}(\mathrm{t}), \mathrm{s}_{\mathrm{t}-60}(\mathrm{t})\right)$ pode ser obtido armazenando-se as últimas $\mathrm{N} / 6$ amostras medidas. $\mathrm{O}$ avanço de $60^{\circ}\left(\mathrm{s}_{\mathrm{r} 60}(\mathrm{t}), \mathrm{s}_{\mathrm{s} 60}(\mathrm{t}), \mathrm{s}_{\mathrm{t} 60}(\mathrm{t})\right)$ pode ser obtido utilizandose as últimas N/3 amostras medidas, equivalentes a um atraso de $120^{\circ}$, com sinal negativo, ( $\mathrm{N}$ é igual ao número de amostras por ciclo da rede na freqüência fundamental).

A transformação das relações fasoriais para o domínio do tempo são exatas para regime permanente senoidal, desde que a janela de $\mathrm{N}$ amostras esteja sincronizada com os sinais $\left(\mathrm{s}_{\mathrm{r}}(\mathrm{t}), \mathrm{s}_{\mathrm{s}}(\mathrm{t})\right.$ e $\mathrm{s}_{\mathrm{t}}(\mathrm{t})$, onde a janela de amostras impõe um transitório para variações em degrau na amplitude ou fase dos sinais.

$$
\begin{gathered}
\left.\left[\begin{array}{l}
s_{r-}(t) \\
s_{s-}(t) \\
s_{t-}(t)
\end{array}\right]=-\frac{1}{3} \cdot\left[\begin{array}{ccc}
-1 & 0 & 0 \\
0 & -1 & 0 \\
0 & 0 & -1
\end{array}\right] \cdot\left[\begin{array}{l}
s_{r}(t) \\
s_{s}(t) \\
s_{t}(t)
\end{array}\right]+\left[\begin{array}{ccc}
0 & 1 & 0 \\
0 & 0 & 1 \\
1 & 0 & 0
\end{array}\right] \cdot\left[\begin{array}{l}
s_{r 60}(t) \\
s_{s 60}(t) \\
s_{t 60}(t)
\end{array}\right]+\left[\begin{array}{ccc}
0 & 0 & 1 \\
1 & 0 & 0 \\
0 & 1 & 0
\end{array}\right] \cdot\left[\begin{array}{l}
s_{r-60}(t) \\
s_{s-60}(t) \\
s_{t-60}(t)
\end{array}\right]\right] \\
{\left[\begin{array}{l}
s_{r-}(t) \\
s_{s-}(t) \\
s_{t-}(t)
\end{array}\right]=\frac{1}{3} \cdot\left[-\left[\begin{array}{ccc}
-1 & 1 / 2 & 1 / 2 \\
1 / 2 & -1 & 1 / 2 \\
1 / 2 & 1 / 2 & -1
\end{array}\right] \cdot\left[\begin{array}{l}
s_{r}(t) \\
s_{s}(t) \\
s_{t}(t)
\end{array}\right]+\left[\begin{array}{ccc}
0 & \frac{\sqrt{3}}{2} & -\frac{\sqrt{3}}{2} \\
-\frac{\sqrt{3}}{2} & 0 & \frac{\sqrt{3}}{2} \\
\frac{\sqrt{3}}{2} & -\frac{\sqrt{3}}{2} & 0
\end{array}\right] \cdot\left[\begin{array}{l}
s_{r-90}(t) \\
s_{s-90}(t) \\
s_{t-90}(t)
\end{array}\right]\right.}
\end{gathered}
$$


O mesmo raciocínio pode ser aplicado para calcular a seqüência positiva obtendose as equações (3.8) e (3.9).

$$
\left.\left[\begin{array}{l}
s_{r+}(t) \\
s_{s+}(t) \\
s_{t+}(t)
\end{array}\right]=-\frac{1}{3} \cdot\left[\begin{array}{ccc}
-1 & 0 & 0 \\
0 & -1 & 0 \\
0 & 0 & -1
\end{array}\right]\left[\begin{array}{l}
s_{r}(t) \\
s_{s}(t) \\
s_{t}(t)
\end{array}\right]+\left[\begin{array}{ccc}
0 & 1 & 0 \\
0 & 0 & 1 \\
1 & 0 & 0
\end{array}\right]\left[\begin{array}{l}
s_{r-60}(t) \\
s_{s-60}(t) \\
s_{t-60}(t)
\end{array}\right]+\left[\begin{array}{ccc}
0 & 0 & 1 \\
1 & 0 & 0 \\
0 & 1 & 0
\end{array}\right]\left[\begin{array}{l}
s_{r 60}(t) \\
s_{s 60}(t) \\
s_{t 60}(t)
\end{array}\right]\right]
$$

$$
\left[\begin{array}{l}
s_{r+}(t) \\
s_{s+}(t) \\
s_{t+}(t)
\end{array}\right]=\frac{1}{3} \cdot\left[-\left[\begin{array}{ccc}
-1 & 1 / 2 & 1 / 2 \\
1 / 2 & -1 & 1 / 2 \\
1 / 2 & 1 / 2 & -1
\end{array}\right] \cdot\left[\begin{array}{l}
s_{r}(t) \\
s_{s}(t) \\
s_{t}(t)
\end{array}\right]-\left[\begin{array}{ccc}
0 & \frac{\sqrt{3}}{2} & -\frac{\sqrt{3}}{2} \\
-\frac{\sqrt{3}}{2} & 0 & \frac{\sqrt{3}}{2} \\
\frac{\sqrt{3}}{2} & -\frac{\sqrt{3}}{2} & 0
\end{array}\right] \cdot\left[\begin{array}{l}
s_{r-90}(t) \\
s_{s-90}(t) \\
s_{t-90}(t)
\end{array}\right]\right]
$$

As operações definidas pelas equações (3.6), (3.7), (3.8) e (3.9) serão chamadas A, B, C e D respectivamente. As operações A e B apresentam o mesmo resultado para a freqüência fundamental, ou seja, extraem os sinais de seqüência negativa. $O$ mesmo ocorre para as operações $\mathbf{C}$ e $\mathbf{D}$, que extraem os sinais de seqüência positiva. Os quatro métodos foram originalmente desenvolvidos para a freqüência fundamental. As componentes harmônicas apresentarão comportamentos diferentes dependendo de sua ordem e seqüência, para as operações A, B, C ou D. A Tabela I e a Tabela II apresentam o ganho de amplitude e fase relativo ao uso das operações A, B, C e D, e suas aplicações em cascata (AB) e (CD) para seqüências positivas e negativas de harmônicas ímpares e pares respectivamente.

TABELA I - Ganho de amplitude (p.u.) e fase (graus) das operações A, B, C, D, AB,

\begin{tabular}{|c|c|c|c|c|c|c|}
\hline operação & A & B & C & D & $A B$ & $C D$ \\
\hline $1^{\circ}$ seq + & & & $1 \longdiv { 0 }$ & $1 \longdiv { 0 }$ & & $1 \longdiv { 0 }$ \\
\hline $1^{\circ}$ seq- & $1 \longdiv { 0 }$ & $1 \longdiv { 0 }$ & 0 & 0 & $1 \longdiv { 0 }$ & 0 \\
\hline $3^{\circ} \mathrm{seq}^{+}$ & 0 & $1 \longdiv { 0 }$ & 0 & 0 & 0 & 0 \\
\hline $3^{\circ}$ seq- & 0 & 0 & 0 & $1 \longdiv { 0 }$ & 0 & 0 \\
\hline $5^{\circ}$ seq $^{+}$ & $1 \longdiv { 0 }$ & 0 & 0 & $1 \longdiv { 0 }$ & 0 & 0 \\
\hline $5^{\circ}$ seq- & 0 & $1 \longdiv { 0 }$ & $1 \longdiv { 0 }$ & 0 & 0 & 0 \\
\hline
\end{tabular}
$C D$ para harmônicas impares de seqüência positiva e negativa. 
TABELA II - Ganho de amplitude (p.u.) e fase (graus) das operações A, B, C, D, AB, CD para harmônicas pares de seqüência positiva e negativa.

\begin{tabular}{|c|c|c|c|c|c|c|}
\hline Operação & $A$ & $B$ & C & D & $A B$ & CD \\
\hline $2^{\circ} \mathrm{seq}+$ & $\frac { 1 } { 3 } \longdiv { 0 }$ & $\frac { 7 } { 1 0 } \longdiv { 4 5 }$ & $\frac { 2 } { 3 } \longdiv { - 6 0 }$ & $\frac { 7 } { 1 0 } \longdiv { - 4 5 }$ & $\frac { 7 } { 3 0 } \longdiv { 4 5 }$ & $\frac { 1 4 } { 3 0 } \longdiv { - 1 0 5 }$ \\
\hline $2^{\circ}$ seq- & $\frac { 2 } { 3 } \longdiv { - 6 0 }$ & $\frac { 7 } { 1 0 } \longdiv { - 4 5 }$ & $\frac { 1 } { 3 } \longdiv { 0 }$ & $\frac{7}{10} \sqrt{45}$ & $\frac { 1 4 } { 3 0 } \longdiv { - 1 0 5 }$ & $\frac{7}{30} \sqrt{45}$ \\
\hline $4^{\circ} \mathrm{seq}^{+}$ & $\frac { 2 } { 3 } \longdiv { 6 0 }$ & $\frac { 7 } { 1 0 } \longdiv { - 4 5 }$ & $\frac { 1 } { 3 } \longdiv { 0 }$ & $\frac { 7 } { 1 0 } \longdiv { 4 5 }$ & $\frac{14}{30} \sqrt{15}$ & $\frac{7}{30} \sqrt[45]{ }$ \\
\hline $4^{\circ}$ seq- & $\frac{1}{3} \sqrt{0}$ & $\frac { 7 } { 1 0 } \longdiv { 4 5 }$ & $\frac{2}{3} \sqrt{60}$ & $\frac { 7 } { 1 0 } \longdiv { - 4 5 }$ & $\frac { 7 } { 3 0 } \longdiv { 4 5 }$ & $\frac{14}{30} \sqrt{15}$ \\
\hline $6^{\circ}$ seq $^{+}$ & $\left.\frac{2}{3}\right)-60$ & $\frac { 7 } { 1 0 } \longdiv { 4 5 }$ & $\frac{2}{3} \sqrt{60}$ & $\frac { 7 } { 1 0 } \longdiv { - 4 5 }$ & $\left.\frac{14}{30}\right)-15$ & $\frac{14}{30} \sqrt{15}$ \\
\hline $6^{\circ}$ seq- & $\frac{2}{3} \sqrt{60}$ & $\frac { 7 } { 1 0 } \longdiv { - 4 5 }$ & $\frac { 2 } { 3 } \longdiv { - 6 0 }$ & $\frac { 7 } { 1 0 } \longdiv { 4 5 }$ & $\frac{14}{30} \sqrt{15}$ & $\frac{14}{30} \sqrt{-15}$ \\
\hline $8^{\circ} \mathrm{seq}+$ & $\frac { 1 } { 3 } \longdiv { 0 }$ & $\frac { 7 } { 1 0 } \longdiv { - 4 5 }$ & $\frac { 2 } { 3 } \longdiv { - 6 0 }$ & $\frac { 7 } { 1 0 } \longdiv { 4 5 }$ & $\frac { 7 } { 3 0 } \longdiv { - 4 5 }$ & $\frac{14}{30} \sqrt{-15}$ \\
\hline $8^{\circ}$ seq- & $\left.\frac{2}{3}\right)-60$ & $\frac { 7 } { 1 0 } \longdiv { 4 5 }$ & $\frac{1}{3} \sqrt{0}$ & $\frac { 7 } { 1 0 } \longdiv { - 4 5 }$ & $\left.\frac{14}{30}\right)-15$ & $\frac { 7 } { 3 0 } \longdiv { - 4 5 }$ \\
\hline $10^{\circ} \mathrm{seq}+$ & $\frac{2}{3} \sqrt{60}$ & $\frac { 7 } { 1 0 } \longdiv { 4 5 }$ & $\frac{1}{3} \sqrt{0}$ & $\frac { 7 } { 1 0 } \longdiv { - 4 5 }$ & $\frac{14}{30} \sqrt{105}$ & $\frac{7}{30} \sqrt{-45}$ \\
\hline $10^{\circ}$ seq- & $\frac { 1 } { 3 } \longdiv { 0 }$ & $\frac { 7 } { 1 0 } \longdiv { - 4 5 }$ & $\frac{2}{3} \sqrt{60}$ & $\frac{7}{10} \sqrt{45}$ & $\frac { 7 } { 3 0 } \longdiv { - 4 5 }$ & $\frac{14}{30} \sqrt{105}$ \\
\hline
\end{tabular}

Para harmônicos impares:

- a operação A apresenta o mesmo ganho para harmônicos de seqüência negativa de ordem $(1+6 n)(n=0,1,2, \ldots)$ e para harmônicos de seqüência positiva de ordem $(5+6 n)$, apresentando ganho nulo para outras situações.

- a operação $\mathbf{C}$ apresenta o mesmo ganho para harmônicos de seqüência positiva de ordem $(1+6 n)(n=0,1,2, \ldots)$ e para harmônicos de seqüência negativa de ordem $(5+6 n)$, apresentando ganho nulo para outras situações.

- a operação B apresenta o mesmo ganho para harmônicos de seqüência positiva de ordem $(3+4 n)$ e seqüência negativa de ordem $(1+4 n)$, apresentando ganho nulo para outras situações.

- a operação D apresenta o mesmo ganho para harmônicos de seqüência negativa de ordem $(3+4 n)$ e seqüência positiva de ordem $(1+4 n)$, apresentando ganho nulo para outras situações.

- a operação cascateada AB apresenta o mesmo ganho para harmônicos de seqüência positiva de ordem $(11+12 n)$ e seqüência negativa de ordem $(1+12 n)$, apresentando ganho nulo para outras situações.

- a operação cascateada CD apresenta o mesmo ganho para harmônicos de seqüência negativa de ordem $(11+12 n)$ e seqüência positiva de ordem $(1+12 n)$, apresentando ganho nulo para outras situações. 
Para harmônicos pares:

- as operações A e C apresentam o mesmo ganho para harmônicos de ordem $(2+6 n),(4+6 n)$ e $(6+6 n)$.

- as operações B e D apresentam o mesmo ganho para harmônicos de ordem $(2+4 n)$ e $(4+4 n)$.

- as operações cascateadas (AB) e (CD) apresentam o mesmo ganho para harmônicos de ordem $(2+12 n),(4+12 n),(6+12 n),(8+12 n),(10+12 n)$ e $(12+12 n)$.

Para todas as operações, os harmônicos pares não são cancelados, mas apenas atenuados. Para obter uma atenuação maior as operações podem ser cascateadas.

A demonstração matemática das operações A, B, C e D para um sinal contendo componentes de seqüência positiva e negativa da fundamental e para um sinal contendo componentes harmônicas de seqüência positiva e negativa é apresentada a seguir.

\subsection{DEMONSTRAÇÃO MATEMÁTICA DA OPERAÇÃO “A” APLICADA A UM SINAL SENOIDAL E PERIÓDICO QUALQUER}

Hipótese a ser provada: a eq.(3.6) é válida para o domínio do tempo, em regime permanente.

A validação desta hipótese é feita adotando-se três sinais instantâneos $s_{r}(t), s_{s}(t) e$ $\mathrm{s}_{\mathrm{t}}(\mathrm{t})$, periódicos e senoidais, contendo componentes de seqüência positiva e negativa e calculando-se as componentes de seqüência negativa pela eq.(3.6);

Os resultados esperados são três sinais representando a seqüência negativa do sinal original.

Assim, sejam os sinais instantâneos $s_{r}(t), s_{s}(t)$ e $s_{t}(t)$ representados na forma exponencial eqs.(3.1-1), (3.1-2), (3.1-3), os sinais instantâneos adiantados de $\pi / 3$, (3.1-4), (3.1-5) e (3.1-6) e os sinais instantâneos atrasados de $\pi / 3$, (3.1-7), (3.1-8) e (3.1-9). 


$$
\begin{gathered}
S_{r}(t)=\operatorname{Re}\left(S_{+} \cdot e^{j\left(\omega t+\phi^{+}\right)}+S_{-} \cdot e^{j\left(-\omega t+\phi^{-}\right)}\right) \\
s_{s}(t)=\operatorname{Re}\left(S_{+} \cdot e^{j\left(\omega t+\phi^{+}-\frac{2 . \pi}{3}\right)}+S_{-} \cdot e^{j\left(-\omega t+\phi^{-}+\frac{2 \cdot \pi}{3}\right)}\right) \\
s_{t}(t)=\operatorname{Re}\left(S_{+} \cdot e^{j\left(\omega t+\phi^{+}+\frac{2 \cdot \pi}{3}\right)}+S_{-} \cdot e^{j\left(-\omega t+\phi^{-}-\frac{2 . \pi}{3}\right)}\right) \\
S_{r 60}(t)=\operatorname{Re}\left(S_{+} \cdot e^{j\left(\omega t+\phi^{+}+\frac{\pi}{3}\right)}+S_{-} \cdot e^{j\left(-\omega t+\phi^{-}+\frac{\pi}{3}\right)}\right) \\
S_{s 60}(t)=\operatorname{Re}\left(S_{+} \cdot e^{j\left(\omega t+\phi^{+}-\frac{2 \cdot \pi}{3}+\frac{\pi}{3}\right)}+S_{-} \cdot e^{j\left(-\omega t+\phi^{-}+\frac{2 \cdot \pi}{3}+\frac{\pi}{3}\right)}\right) \\
S_{t 60}(t)=\operatorname{Re}\left(S_{+} \cdot e^{j\left(\omega t+\phi^{+}+\frac{2 \cdot \pi}{3}+\frac{\pi}{3}\right)}+S_{-} \cdot e^{j\left(-\omega t+\phi^{-}-\frac{2 \cdot \pi}{3}+\frac{\pi}{3}\right)}\right) \\
S_{r-60}(t)=\operatorname{Re}\left(S_{+} \cdot e^{j\left(\omega t+\phi^{+}-\frac{\pi}{3}\right)}+S_{-} \cdot e^{j\left(-\omega t+\phi^{-}-\frac{\pi}{3}\right)}\right) \\
S_{s-60}(t)=\operatorname{Re}\left(S_{+} \cdot e^{j\left(\omega t+\phi^{+}-\frac{2 \cdot \pi}{3}-\frac{\pi}{3}\right)}+S_{-} \cdot e^{j\left(-\omega t+\phi^{-}+\frac{2 \cdot \pi}{3}-\frac{\pi}{3}\right)}\right) \\
S_{t-60}(t)=\operatorname{Re}\left(S_{+} \cdot e^{j\left(\omega t+\phi^{+}+\frac{2 \cdot \pi}{3}-\frac{\pi}{3}\right)}+S_{-} \cdot e^{j\left(-\omega t+\phi^{-}-\frac{2 \cdot \pi}{3}-\frac{\pi}{3}\right)}\right)
\end{gathered}
$$

Calculam-se individualmente pela eq.(3.6) os três sinais de seqüência negativa comprovando assim a validação da hipótese inicial.

A seguir descreve-se o cálculo de $s_{r-}(t)$ (eq.(3.1-10)) substituindo os valores dados pelas eqs. (3.1-1) a (3.1-9) na eq. (3.6).

$$
\begin{aligned}
& S_{r-}(t)=\operatorname{Re}(\underbrace{+\frac{S_{+}}{3} \cdot e^{j\left(\omega t+\phi^{+}\right)}}_{\text {termo } 1} \underbrace{\frac{S_{-}}{3} \cdot e^{j\left(-\omega t+\phi^{-}\right)}}_{\text {termo } 2} \underbrace{-\frac{S_{+}}{3} \cdot e^{j\left(\omega t+\phi^{+}-\frac{2 \cdot \pi}{3}+\frac{\pi}{3}\right)}}_{\text {termo } 3}- \\
& \underbrace{\frac{S_{-}}{3} \cdot e^{j\left(-\omega t+\phi^{-}+\frac{2 \cdot \pi}{3}+\frac{\pi}{3}\right)}}_{\text {termo } 4} \underbrace{-\frac{S_{+}}{3} \cdot e^{j\left(\omega t+\phi^{+}+\frac{2 \cdot \pi}{3}-\frac{\pi}{3}\right)}}_{\text {termo } 5} \underbrace{-\frac{S_{-}}{3} \cdot e^{j\left(-\omega t+\phi^{-}-\frac{2 \cdot \pi}{3}-\frac{\pi}{3}\right)}}_{\text {termo } 6})=\operatorname{Re}\left(S_{-} \cdot e^{j\left(-\omega t+\phi^{-}\right)}\right)
\end{aligned}
$$

Os sinais extraídos $\mathrm{s}_{\mathrm{s}-}(\mathrm{t})$ e $\mathrm{s}_{\mathrm{t}-}(\mathrm{t})$ seguem $\mathrm{o}$ mesmo procedimento e são apresentados nas eqs. (3.1-11) e (3.1-12).

$$
\begin{array}{r}
S_{s-}(t)=\operatorname{Re}\left(S_{-} \cdot e^{j\left(-\omega t+\phi^{-}+\frac{2 \pi}{3}\right)}\right) \\
S_{t-}(t)=\operatorname{Re}\left(S_{-} \cdot e^{j\left(-\omega t+\phi^{-}-\frac{2 \pi}{3}\right)}\right)
\end{array}
$$


Apresenta-se a seguir um quadro resumo (Tabela III) contendo o sinal original e o sinal de seqüência negativa obtido comprovando assim a hipótese inicial.

Tabela III - Quadro demonstrativo dos sinais extraídos - operação A

\begin{tabular}{|c|c|}
\hline Sinal Original & Sinal extraído \\
\hline$S_{r}(t)=\operatorname{Re}\left(S_{+} \cdot e^{j\left(\omega t+\phi^{+}\right)}+S_{-} \cdot e^{j\left(-\omega t+\phi^{-}\right)}\right)$ & $S_{r-}(t)=\operatorname{Re}\left(S_{-} \cdot e^{j\left(-\omega t+\phi^{-}\right)}\right)$ \\
\hline$S_{s}(t)=\operatorname{Re}\left(S_{+} \cdot e^{j\left(\omega t+\phi^{+}-\frac{2 \cdot \pi}{3}\right)}+S_{-} \cdot e^{j\left(-\omega t+\phi^{-}+\frac{2 \cdot \pi}{3}\right)}\right)$ & $S_{s-}(t)=\operatorname{Re}\left(S_{-} \cdot e^{j\left(-\omega t+\phi^{-}+\frac{2 \pi}{3}\right)}\right)$ \\
\hline$S_{t}(t)=\operatorname{Re}\left(S_{+} \cdot e^{j\left(\omega t+\phi^{+}+\frac{2 . \pi}{3}\right)}+S_{-} \cdot e^{j\left(-\omega t+\phi^{-}-\frac{2 . \pi}{3}\right)}\right)$ & $S_{t-}(t)=\operatorname{Re}\left(S_{-} \cdot e^{j\left(-\omega t+\phi^{-}-\frac{2 \pi}{3}\right)}\right)$ \\
\hline
\end{tabular}

\subsection{DEMONSTRAÇÃO MATEMÁTICA DA OPERAÇÃO “B” APLICADA A UM SINAL SENOIDAL E PERIÓDICO QUALQUER}

Hipótese a ser provada: a eq.(3.7) é válida para o domínio do tempo, em regime permanente.

A validação desta hipótese é feita adotando-se três sinais instantâneos $s_{r}(t), s_{s}(t) e$ $\mathrm{s}_{\mathrm{t}}(\mathrm{t})$, periódicos e senoidais, contendo componentes de seqüência positiva e negativa e calculando-se as componentes de seqüência negativa pela eq.(3.7);

Os resultados esperados são três sinais representando a seqüência negativa do sinal original.

Assim, sejam os sinais instantâneos $\mathrm{s}_{\mathrm{r}}(\mathrm{t}), \mathrm{s}_{\mathrm{s}}(\mathrm{t})$ e $\mathrm{s}_{\mathrm{t}}(\mathrm{t})$, respectivamente eqs.(3.2-1), (3.2-2) e (3.2-3) e as correntes instantâneas atrasadas de m/2 eqs.(3.2-4), (3.2-5) e $(3.2-6)$.

$$
\begin{aligned}
& s_{r}(t)=S_{+} \cdot \cos \left(\omega t+\phi^{+}\right)+S_{-} \cdot \cos \left(-\omega t+\phi^{-}\right) \\
& s_{s}(t)=S_{+} \cdot \cos \left(\omega t+\phi^{+}-\frac{2 \cdot \pi}{3}\right)+S_{-} \cdot \cos \left(-\omega t+\phi^{-}+\frac{2 \cdot \pi}{3}\right) \\
& s_{t}(t)=S_{+} \cdot \cos \left(\omega t+\phi^{+}+\frac{2 \cdot \pi}{3}\right)+S_{-} \cdot \cos \left(-\omega t+\phi^{-}-\frac{2 \cdot \pi}{3}\right)
\end{aligned}
$$




$$
\begin{gathered}
S_{r-90}(t)=S_{+} \cdot \cos \left(\omega t+\phi^{+}-\frac{\pi}{2}\right)+S_{-} \cdot \cos \left(-\omega t+\phi^{-}-\frac{\pi}{2}\right) \\
S_{s-90}(t)=S_{+} \cdot \cos \left(\omega t+\phi^{+}-\frac{2 \cdot \pi}{3}-\frac{\pi}{2}\right)+S_{-} \cdot \cos \left(-\omega t+\phi^{-}+\frac{2 \cdot \pi}{3}-\frac{\pi}{2}\right) \\
S_{t-90}(t)=S_{+} \cdot \cos \left(\omega t+\phi^{+}+\frac{2 \cdot \pi}{3}-\frac{\pi}{2}\right)+S_{-} \cdot \cos \left(-\omega t+\phi^{-}-\frac{2 \cdot \pi}{3}-\frac{\pi}{2}\right)
\end{gathered}
$$

Representando as equações acima na forma exponencial obtemos as eqs.(3.2-7), (3.2-8), (3.2-9), (3.2-10), (3.2-11) e (3.2-12).

$$
\begin{gathered}
S_{r}(t)=\operatorname{Re}\left(S_{+} \cdot e^{j\left(\omega t+\phi^{+}\right)}+S_{-} \cdot e^{j\left(-\omega t+\phi^{-}\right)}\right) \\
S_{s}(t)=\operatorname{Re}\left(S_{+} \cdot e^{j\left(\omega t+\phi^{+}-\frac{2 \cdot \pi}{3}\right)}+S_{-} \cdot e^{j\left(-\omega t+\phi^{-}+\frac{2 \cdot \pi}{3}\right)}\right) \\
S_{t}(t)=\operatorname{Re}\left(S_{+} \cdot e^{j\left(\omega t+\phi^{+}+\frac{2 \cdot \pi}{3}\right)}+S_{-} \cdot e^{j\left(-\omega t+\phi^{-}-\frac{2 \cdot \pi}{3}\right)}\right) \\
S_{r-90}(t)=\operatorname{Re}\left(S_{+} \cdot e^{j\left(\omega t+\phi^{+}-\frac{\pi}{2}\right)}+S_{-} \cdot e^{j\left(-\omega t+\phi^{-}-\frac{\pi}{2}\right)}\right) \\
S_{s-90}(t)=\operatorname{Re}\left(S_{+} \cdot e^{j\left(\omega t+\phi^{+}-\frac{2 \cdot \pi}{3}-\frac{\pi}{2}\right)}+S_{-} \cdot e^{j\left(-\omega t+\phi^{-}+\frac{2 \cdot \pi}{3}-\frac{\pi}{2}\right)}\right) \\
S_{t-90}(t)=\operatorname{Re}\left(S_{+} \cdot e^{j\left(\omega t+\phi^{+}+\frac{2 \cdot \pi}{3}-\frac{\pi}{2}\right)}+S_{-} \cdot e^{j\left(-\omega t+\phi^{-}-\frac{2 \cdot \pi}{3}-\frac{\pi}{2}\right)}\right)
\end{gathered}
$$

Calculam-se individualmente pela eq.(3.7) os três sinais $\left(s_{r_{-}}(t), s_{s^{-}}(t), s_{t-}(t)\right)$ comprovando assim a validação da hipótese inicial.

A seguir descreve-se o cálculo de $s_{r-}(t)$ (eq.(3.2-13)) substituindo os valores dados pelas eqs. (3.2-7), (3.2-8), (3.2-9), (3.2-10), (3.2-11) e (3.2-12) na eq.(3.7). 


$$
\begin{aligned}
& S_{r-}(t)=\operatorname{Re}(\underbrace{\frac{S_{+}}{3} \cdot e^{j\left(\omega t+\phi^{+}\right)}}_{\text {termo } 1}+\underbrace{\frac{S_{-}}{3} \cdot e^{j\left(-\omega t+\phi^{-}\right)}}_{\text {termo } 2} \underbrace{-\frac{S_{+}}{6} \cdot e^{j\left(\omega t+\phi^{+}-\frac{2 \cdot \pi}{3}\right)}}_{\text {termo } 3}- \\
& \underbrace{\frac{S_{-}}{6} \cdot e^{j\left(-\omega t+\phi^{-}+\frac{2 \cdot \pi}{3}\right)}}_{\text {termo } 4}-\underbrace{-\frac{S_{+}}{6} \cdot e^{j\left(\omega t+\phi^{+}+\frac{2 \cdot \pi}{3}\right)}}_{\text {termo } 5} \underbrace{-\frac{S_{-}}{6} \cdot e^{j\left(-\omega t+\phi^{-}-\frac{2 . \pi}{3}\right)}}_{\text {termo } 6}+ \\
& \underbrace{\frac{\sqrt{3}}{6} \cdot S_{+} \cdot e^{j\left(\omega t+\phi^{+}-\frac{2 \cdot \pi}{3}-\frac{\pi}{2}\right)}}_{\text {termo } 7}+\underbrace{+\frac{\sqrt{3}}{6} \cdot S_{-} \cdot e^{j\left(-\omega t+\phi^{-}+\frac{2 \cdot \pi}{3}-\frac{\pi}{2}\right)}}_{\text {termo } 8}- \\
& \underbrace{\frac{\sqrt{3}}{6} \cdot S_{+} \cdot e^{j\left(\omega t+\phi^{+}+\frac{2 \cdot \pi}{3}-\frac{\pi}{2}\right)}}_{\text {termo } 9} \underbrace{\left.\left.-\frac{\sqrt{3}}{6} \cdot S_{-} \cdot e^{j\left(-\omega t+\phi^{-}-\frac{2 \cdot \pi}{3}-\frac{\pi}{2}\right)}\right)\right)}_{\text {termo } 10}
\end{aligned}
$$

Utilizando-se a identidade $\cos (\alpha)=\frac{\mathrm{e}^{\mathrm{j} \cdot \alpha}+\mathrm{e}^{-\mathrm{j} . \alpha}}{2}$ para os termos (3) e (5) da eq.(3.213), bem como para os termos (4) e (6), obtêm-se as equações (3.2-14) e (3.2-15).

$$
\begin{aligned}
& \text { Termo } 3+\text { termo } 5=-\frac{S_{+}}{6} \cdot e^{j\left(\omega t+\phi^{+}-\frac{2 \cdot \pi}{3}\right)}-\frac{S_{+}}{6} \cdot e^{j\left(\omega t+\phi^{+}+\frac{2 \cdot \pi}{3}\right)}= \\
& =-\frac{S_{+}}{6} \cdot e^{j\left(\omega t+\phi^{+}\right)} \cdot 2 \cdot \cos \left(\frac{2 \cdot \pi}{3}\right)=\frac{S_{+}}{6} \cdot e^{j\left(\omega t+\phi^{+}\right)} \\
& \text {Termo } 4+\text { termo } 6=-\frac{S_{-}}{6} \cdot e^{j\left(-\omega t+\phi^{-}+\frac{2 \cdot \pi}{3}\right)}-\frac{S_{-}}{6} \cdot e^{j\left(-\omega t+\phi^{-}-\frac{2 \cdot \pi}{3}\right)}= \\
& =-\frac{S_{-}}{6} \cdot e^{j\left(-\omega t+\phi^{-}\right)} \cdot 2 \cdot \cos \left(\frac{2 \cdot \pi}{3}\right)=+\frac{S_{-}}{6} \cdot e^{j\left(-\omega t+\phi^{-}\right)}
\end{aligned}
$$

Utilizando-se a identidade $\sin (\alpha)=\frac{\mathrm{e}^{\mathrm{j} \cdot \alpha}-\mathrm{e}^{-\mathrm{j} . \alpha}}{2 . \mathrm{j}}$ para os termos (7) e (9) da eq.(3.213), bem como para os termos (8) e (10), obtêm-se as equações (3.2-16) e (3.2-17).

$$
\begin{aligned}
& \text { Termo } 7+\text { Termo } 9=+\frac{\sqrt{3}}{6} \cdot S_{+} \cdot e^{j\left(\omega t+\phi^{+}-\frac{2 \cdot \pi}{3}-\frac{\pi}{2}\right)}-\frac{\sqrt{3}}{6} \cdot S_{+} \cdot e^{j\left(\omega t+\phi^{+}+\frac{2 \cdot \pi}{3}-\frac{\pi}{2}\right)}= \\
& -\frac{\sqrt{3}}{6} \cdot S_{+} \cdot e^{j\left(\omega t+\phi^{+}-\frac{\pi}{2}\right)} \cdot 2 \cdot j \cdot \sin \left(+\frac{2 \cdot \pi}{3}\right)= \\
& -\frac{S_{+}}{2} \cdot e^{j\left(\omega t+\phi^{+}-\frac{\pi}{2}\right)} \cdot e^{j\left(+\frac{\pi}{2}\right)}=-\frac{S_{+}}{2} \cdot e^{j\left(\omega t+\phi^{+}\right)}
\end{aligned}
$$




$$
\begin{aligned}
& \text { Termo } 8+T \text { ermo } 10=-\frac{\sqrt{3}}{6} \cdot S_{-} \cdot e^{j\left(-\omega t+\phi^{-}+\frac{2 \cdot \pi}{3}-\frac{\pi}{2}\right)}+\frac{\sqrt{3}}{6} \cdot S_{-} \cdot e^{j\left(-\omega t+\phi^{-}-\frac{2 \cdot \pi}{3}-\frac{\pi}{2}\right)}= \\
& -\frac{\sqrt{3}}{6} \cdot S_{-} \cdot e^{j\left(-\omega t+\phi^{-}-\frac{\pi}{2}\right)} \cdot 2 \cdot j \cdot \sin \left(\frac{-2 \cdot \pi}{3}\right)=+\frac{1}{2} \cdot S_{-} \cdot e^{j\left(-\omega t+\phi^{-}-\frac{\pi}{2}\right)} \cdot e^{j\left(+\frac{\pi}{2}\right)}= \\
& +\frac{1}{2} \cdot S_{-} \cdot e^{j\left(-\omega t+\phi^{-}\right)}
\end{aligned}
$$

Substituindo-se os resultados das eqs.(3.2-14), (3.2-15), (3.2-16) e (3.2-17) na eq.(3.2-13) obtém-se a parcela da corrente de seqüência negativa instantânea da fase $r$ na eq.(3.2-18).

$$
s_{r-}(t)=\operatorname{Re}\left(+\frac{S_{+}}{2} \cdot e^{j\left(\omega t+\phi^{+}\right)}+\frac{S_{-}}{2} \cdot e^{j\left(-\omega t+\phi^{-}\right)}-\frac{S_{+}}{2} \cdot e^{j\left(\omega t+\phi^{+}\right)}+\frac{S_{-}}{2} \cdot e^{j\left(-\omega t+\phi^{-}\right)}\right)=\operatorname{Re}\left(+S_{-} \cdot e^{j\left(-\omega t+\phi^{-}\right)}\right)
$$

Os sinais $\mathrm{s}_{\mathrm{s}-}(\mathrm{t})$ e $\mathrm{s}_{\mathrm{t}-\mathrm{t}}(\mathrm{t})$ seguem o mesmo procedimento e são apresentadas nas eqs.(3.2-19) e (3.2-20).

$$
\begin{aligned}
& S_{s^{-}}(t)=\operatorname{Re}\left(S_{-} \cdot e^{j\left(-\omega t+\phi^{-}+\frac{2 \pi}{3}\right)}\right) \\
& S_{t-}(t)=\operatorname{Re}\left(S_{-} \cdot e^{j\left(-\omega t+\phi^{-}-\frac{2 \pi}{3}\right)}\right)
\end{aligned}
$$

Apresenta-se a seguir um quadro resumo (Tabela IV) contendo o sinal original e o sinal de seqüência negativa obtido comprovando assim a hipótese inicial.

Tabela IV - Quadro demonstrativo dos sinais extraídos - operação B

\begin{tabular}{|l|l|}
\hline Sinal Original & Sinal extraído \\
\hline$S_{r}(t)=\operatorname{Re}\left(S_{+} \cdot e^{j\left(\omega t+\phi^{+}\right)}+S_{-} \cdot e^{j\left(-\omega t+\phi^{-}\right)}\right)$ & $S_{r-}(t)=\operatorname{Re}\left(+S_{-} \cdot e^{j\left(-\omega t+\phi^{-}\right)}\right)$ \\
\hline$S_{s}(t)=\operatorname{Re}\left(S_{+} \cdot e^{j\left(\omega t+\phi^{+}-\frac{2 \cdot \pi}{3}\right)}+S_{-} \cdot e^{j\left(-\omega t+\phi^{-}+\frac{2 \cdot \pi}{3}\right)}\right)$ & $S_{s-}(t)=\operatorname{Re}\left(S_{-} \cdot e^{j\left(-\omega t+\phi^{-}+\frac{2 \pi}{3}\right)}\right)$ \\
\hline$S_{t}(t)=\operatorname{Re}\left(S_{+} \cdot e^{j\left(\omega t+\phi^{+}+\frac{2 \cdot \pi}{3}\right)}+S_{-} \cdot e^{j\left(-\omega t+\phi^{-}-\frac{2 \cdot \pi}{3}\right)}\right)$ & $S_{t-}(t)=\operatorname{Re}\left(S_{-} \cdot e^{j\left(-\omega t+\phi^{-}-\frac{2 \pi}{3}\right)}\right)$ \\
\hline
\end{tabular}

De forma análoga, as demonstrações dos itens A.1 e A.2 valem respectivamente para as operações C e D. 


\subsection{DEMONSTRAÇÃO MATEMÁTICA DAS OPERAÇÕES APLICADAS A UM SINAL COM CONTEÚDO HARMÔNICO}

Se o sinal analisado contiver harmônicos estes poderão ser extraídos dependendo de sua ordem, seqüência e da operação utilizada (A, B, C, D).

A prova matemática desta constatação é feita adotando-se um sinal trifásico contendo um componente harmônico, periódico e senoidal, qualquer e calculando-se o sinal extraído de acordo com a operação proposta.

Assim, sejam os sinais instantâneos $s_{r h}(t), s_{s h}(t)$ e $s_{t h}(t)$ representados na forma exponencial respectivamente nas eqs.(3.3-1), (3.3-2) e (3.3-3).

$$
\begin{aligned}
& S_{r h}(t)=\operatorname{Re}\left(S_{+} \cdot e^{j\left(h \omega t+\phi^{h+}\right)}+S_{-} \cdot e^{j\left(-h \omega t+\phi^{h-}\right)}\right) \\
& S_{s h}(t)=\operatorname{Re}\left(S_{+} \cdot e^{j\left(h \omega t+\phi^{h+}-\frac{2 \cdot \pi}{3}\right)}+S_{-} \cdot e^{j\left(-h \omega t+\phi^{h-}+\frac{2 \cdot \pi}{3}\right)}\right) \\
& S_{t h}(t)=\operatorname{Re}\left(S_{+} \cdot e^{j\left(h \omega t+\phi^{h+}+\frac{2 \cdot \pi}{3}\right)}+S_{-} \cdot e^{j\left(-h \omega t+\phi^{h-}-\frac{2 . \pi}{3}\right)}\right)
\end{aligned}
$$

\subsubsection{DEMONSTRAÇÃO MATEMÁTICA DA OPERAÇÃO “A” APLICADA A UM SINAL COM CONTEÚDO HARMÔNICO}

Hipótese a ser provada: a eq.(3.6) é válida para o domínio do tempo, em regime permanente para um sinal contendo harmônicos.

A seguir descreve-se o cálculo de $s_{r_{-}}(t)$ (eq.(3.3.1-1)) substituindo os valores dados pelas eqs. (3.3-1), (3.3-2), (3.3-3) na eq.(3.6).

$$
\begin{aligned}
& S_{r-}(t)=\operatorname{Re}\left(+\frac{S_{+}}{3} \cdot e^{j\left(h \omega t+\phi^{h+}\right)}+\frac{S_{-}}{3} \cdot e^{j\left(-h \omega t+\phi^{h-}\right)}+\frac{S_{+}}{3} \cdot e^{j\left(h \omega t+\phi^{h+}-\frac{2 \cdot \pi}{3}-h \cdot 2 \frac{\pi}{3}\right)}+\right. \\
& \left.\frac{S_{-}}{3} \cdot e^{j\left(-h \omega t+\phi^{h-}+\frac{2 \cdot \pi}{3}-h \cdot 2 \frac{\pi}{3}\right)}-\frac{S_{+}}{3} \cdot e^{j\left(h \omega t+\phi^{h+}+\frac{2 \cdot \pi}{3}-h \cdot \frac{\pi}{3}\right)}-\frac{S_{-}}{3} \cdot e^{j\left(-h \omega t+\phi^{h-}-\frac{2 \cdot \pi}{3}-h \cdot \frac{\pi}{3}\right)}\right)
\end{aligned}
$$

Os sinais extraídos $\mathrm{s}_{\mathrm{s}-}(\mathrm{t})$ e $\mathrm{s}_{\mathrm{t}-}(\mathrm{t})$ seguem $\mathrm{o}$ mesmo procedimento e são apresentados nas eqs. (3.3.1-2) e (3.3.1-3). 


$$
\begin{aligned}
& S_{s-}(t)=\operatorname{Re}\left(+\frac{S_{+}}{3} \cdot e^{j\left(h \omega t+\phi^{h+}-\frac{2 \cdot \pi}{3}\right)}+\frac{S_{-}}{3} \cdot e^{j\left(-h \omega t+\phi^{h-}+\frac{2 \cdot \pi}{3}\right)}+\frac{S_{+}}{3} \cdot e^{j\left(h \omega t+\phi^{h+}+\frac{2 \cdot \pi}{3}-h \cdot 2 \cdot \frac{\pi}{3}\right)}+\right. \\
& \left.\frac{S_{-}}{3} \cdot e^{j\left(-h \omega t+\phi^{h-}-\frac{2 \cdot \pi}{3}-h \cdot 2 \cdot \frac{\pi}{3}\right)}-\frac{S_{+}}{3} \cdot e^{j\left(h \omega t+\phi^{h+}-h \cdot \frac{\pi}{3}\right)}-\frac{S_{-}}{3} \cdot e^{j\left(-h \omega t+\phi^{h-}-h \cdot \frac{\pi}{3}\right)}\right)
\end{aligned}
$$

$$
\begin{aligned}
& S_{t-}(t)=\operatorname{Re}\left(+\frac{S_{+}}{3} \cdot e^{j\left(h \omega t+\phi^{h+}+\frac{2 \cdot \pi}{3}\right)}+\frac{S_{-}}{3} \cdot e^{j\left(-h \omega t+\phi^{h-}-\frac{2 \cdot \pi}{3}\right)}+\frac{S_{+}}{3} \cdot e^{j\left(h \omega t+\phi^{h+}-h \cdot 2 \cdot \frac{\pi}{3}\right)}+\right. \\
& \left.\frac{S_{-}}{3} \cdot e^{j\left(-h \omega t+\phi^{-}-h \cdot 2 \cdot \frac{\pi}{3}\right)}-\frac{S_{+}}{3} \cdot e^{j\left(h \omega t+\phi^{h+}-\frac{2 \cdot \pi}{3}-h \cdot \frac{\pi}{3}\right)}-\frac{S_{-}}{3} \cdot e^{j\left(-h \omega t+\phi^{h-}+\frac{2 \cdot \pi}{3}-h \cdot \frac{\pi}{3}\right)}\right)
\end{aligned}
$$

Apresenta-se a seguir um exemplo de cálculo dos sinais extraídos (Tabela V) para harmônicos de $2^{\circ}, 3^{\circ}$ e $5^{\circ}$ ordem de seqüência positiva e negativa comprovando

\begin{tabular}{|c|c|}
\hline Sinal Original $-2^{\circ}$ Ordem & Sinal extraído \\
\hline $\begin{array}{l}S_{r 2}(t)=\operatorname{Re}\left(S_{+} \cdot e^{j\left(2 \omega t+\phi^{2+}\right)}+\right. \\
\left.S_{-} \cdot e^{j\left(-2 \omega t+\phi^{2-}\right)}\right)\end{array}$ & $\begin{array}{l}S_{r-}(t)=\operatorname{Re}\left(+\frac{S_{+}}{3} \cdot e^{j\left(2 \omega t+\phi^{2+}\right.}\right)+\frac{S_{-}}{3} \cdot e^{j\left(-2 \omega t+\phi^{2-}\right)} \\
+\frac{S_{+}}{3} \cdot e^{j\left(2 \omega t+\phi^{2+}-\frac{2 \cdot \pi}{3}-2 \cdot 2 \frac{\pi}{3}\right)}+\frac{S_{-}}{3} \cdot e^{j\left(-2 \omega t+\phi^{2-}+\frac{2 \cdot \pi}{3}-2 \cdot 2 \frac{\pi}{3}\right)} \\
\left.-\frac{S_{+}}{3} \cdot e^{j\left(2 \omega t+\phi^{2+}+\frac{2 \cdot \pi}{3}-2 \cdot \frac{\pi}{3}\right)}-\frac{S_{-}}{3} \cdot e^{j\left(-2 \omega t+\phi^{2-}-\frac{2 \cdot \pi}{3}-2 \cdot \frac{\pi}{3}\right)}\right)= \\
\operatorname{Re}\left(\frac{S_{+}}{3} \cdot e^{j\left(2 \omega t+\phi^{2+}\right)}+\frac{2 \cdot S_{-}}{3} \cdot e^{j\left(-2 \omega t+\phi^{2-}-\frac{\pi}{3}\right)}\right)\end{array}$ \\
\hline $\begin{array}{l}S_{s 2}(t)=\operatorname{Re}\left(S_{+} \cdot e^{j\left(2 \omega t+\phi^{2+}-\frac{2 \cdot \pi}{3}\right)}+\right. \\
\left.S_{-} \cdot e^{j\left(-2 \omega t+\phi^{2-}+\frac{2 . \pi}{3}\right)}\right)\end{array}$ & $\begin{array}{l}S_{s-}(t)=\operatorname{Re}\left(+\frac{S_{+}}{3} \cdot e^{j\left(2 \omega t+\phi^{2+}-\frac{2 \cdot \pi}{3}\right)}+\frac{S_{-}}{3} \cdot e^{j\left(-2 \omega t+\phi^{2-}+\frac{2 \cdot \pi}{3}\right)}+\right. \\
\frac{S_{+}}{3} \cdot e^{j\left(2 \omega t+\phi^{2+}+\frac{2 \cdot \pi}{3}-2 \cdot 2 \cdot \frac{\pi}{3}\right)}+\frac{S_{-}}{3} \cdot e^{j\left(-2 \omega t+\phi^{2-}-\frac{2 \cdot \pi}{3}-2 \cdot 2 \cdot \frac{\pi}{3}\right)} \\
\left.-\frac{S_{+}}{3} \cdot e^{j\left(2 \omega t+\phi^{2+}-2 \cdot \frac{\pi}{3}\right)}-\frac{S_{-}}{3} \cdot e^{j\left(-2 \omega t+\phi^{2-}-2 \cdot \frac{\pi}{3}\right)}\right)= \\
\operatorname{Re}\left(+\frac{S_{+}}{3} \cdot e^{j\left(2 \omega t+\phi^{2+}-\frac{2 \cdot \pi}{3}\right)}+\frac{2 \cdot S_{-}}{3} \cdot e^{j\left(-2 \omega t+\phi^{2-}+\frac{2 \cdot \pi}{3}-\frac{\pi}{3}\right)}\right.\end{array}$ \\
\hline
\end{tabular}
assim a hipótese inicial e validando a Tabela I e II.

Tabela V - Quadro demonstrativo dos sinais extraídos - operação A (com harmônicos) 


\begin{tabular}{|c|c|}
\hline $\begin{array}{l}S_{t 2}(t)=\operatorname{Re}\left(S_{+} \cdot e^{j\left(2 \omega t+\phi^{2+}+\frac{2 . \pi}{3}\right)}+\right. \\
\left.S_{-} \cdot e^{j\left(-2 \omega t+\phi^{2-}-\frac{2 . \pi}{3}\right)}\right)\end{array}$ & $\begin{array}{l}S_{t-}(t)=\operatorname{Re}\left(+\frac{S_{+}}{3} \cdot e^{j\left(2 \omega t+\phi^{2+}+\frac{2 \cdot \pi}{3}\right)}+\frac{S_{-}}{3} \cdot e^{j\left(-2 \omega t+\phi^{2-}-\frac{2 \cdot \pi}{3}\right)}+\right. \\
\frac{S_{+}}{3} \cdot e^{j\left(2 \omega t+\phi^{2+}-2 \cdot 2 \cdot \frac{\pi}{3}\right)}+\frac{S_{-}}{3} \cdot e^{j\left(-2 \omega t+\phi^{-}-2 \cdot 2 \cdot \frac{\pi}{3}\right)}- \\
\left.\frac{S_{+}}{3} \cdot e^{j\left(2 \omega t+\phi^{2+}-\frac{2 \cdot \pi}{3}-2 \cdot \frac{\pi}{3}\right)}-\frac{S_{-}}{3} \cdot e^{j\left(-2 \omega t+\phi^{2-}+\frac{2 \cdot \pi}{3}-2 \cdot \frac{\pi}{3}\right)}\right)= \\
\operatorname{Re}\left(\frac{S_{+}}{3} \cdot e^{j\left(2 \omega t+\phi^{2+}+\frac{2 \cdot \pi}{3}\right)}+\frac{2 \cdot S_{-}}{3} \cdot e^{j\left(-2 \omega t+\phi^{2-}-\frac{2 \cdot \pi}{3}-\frac{\pi}{3}\right)}\right.\end{array}$ \\
\hline Sinal Original $-3^{\circ}$ Ordem & Sinal extraído \\
\hline $\begin{array}{l}S_{r 3}(t)=\operatorname{Re}\left(S_{+} \cdot e^{j\left(3 \omega t+\phi^{3+}\right)}+\right. \\
\left.S_{-} \cdot e^{j\left(-3 \omega t+\phi^{3-}\right)}\right)\end{array}$ & $\begin{array}{l}S_{r-}(t)=\operatorname{Re}\left(+\frac{S_{+}}{3} \cdot e^{j\left(3 \omega t+\phi^{3+}\right)}+\frac{S_{-}}{3} \cdot e^{j\left(-3 \omega t+\phi^{3-}\right)}+\right. \\
\frac{S_{+}}{3} \cdot e^{j\left(3 \omega t+\phi^{3+}-\frac{2 \cdot \pi}{3}-3 \cdot 2 \frac{\pi}{3}\right)}+\frac{S_{-}}{3} \cdot e^{j\left(-3 \omega t+\phi^{3-}+\frac{2 \cdot \pi}{3}-3 \cdot 2 \frac{\pi}{3}\right)} \\
\left.-\frac{S_{+}}{3} \cdot e^{j\left(3 \omega t+\phi^{3+}+\frac{2 \cdot \pi}{3}-3 \cdot \frac{\pi}{3}\right)}-\frac{S_{-}}{3} \cdot e^{j\left(-3 \omega t+\phi^{3-}-\frac{2 \cdot \pi}{3}-3 \cdot \frac{\pi}{3}\right)}\right)=0\end{array}$ \\
\hline $\begin{array}{l}S_{s 3}(t)=\operatorname{Re}\left(S_{+} \cdot e^{j\left(3 \omega t+\phi^{3+}-\frac{2 . \pi}{3}\right)}+\right. \\
\left.S_{-} \cdot e^{j\left(-3 \omega t+\phi^{3-}+\frac{2 . \pi}{3}\right)}\right)\end{array}$ & $\begin{array}{l}S_{s-}(t)=\operatorname{Re}\left(+\frac{S_{+}}{3} \cdot e^{j\left(3 \omega t+\phi^{3+}-\frac{2 \cdot \pi}{3}\right)}+\frac{S_{-}}{3} \cdot e^{j\left(-3 \omega t+\phi^{3-}+\frac{2 \cdot \pi}{3}\right)}+\right. \\
\frac{S_{+}}{3} \cdot e^{j\left(3 \omega t+\phi^{3+}+\frac{2 \cdot \pi}{3}-3 \cdot 2 \cdot \frac{\pi}{3}\right)}+\frac{S_{-}}{3} \cdot e^{j\left(-3 \omega t+\phi^{3-}-\frac{2 \cdot \pi}{3}-3 \cdot 2 \cdot \frac{\pi}{3}\right)}- \\
\left.\frac{S_{+}}{3} \cdot e^{j\left(3 \omega t+\phi^{3+}-3 \cdot \frac{\pi}{3}\right)}-\frac{S_{-}}{3} \cdot e^{j\left(-3 \omega t+\phi^{3-}-3 \cdot \frac{\pi}{3}\right)}\right)=0\end{array}$ \\
\hline $\begin{array}{l}S_{t 3}(t)=\operatorname{Re}\left(S_{+} \cdot e^{j\left(3 \omega t+\phi^{3+}+\frac{2 \cdot \pi}{3}\right)}+\right. \\
\left.S_{-} \cdot e^{j\left(-3 \omega t+\phi^{3-}-\frac{2 . \pi}{3}\right)}\right)\end{array}$ & $\begin{array}{l}S_{t-}(t)=\operatorname{Re}\left(+\frac{S_{+}}{3} \cdot e^{j\left(3 \omega t+\phi^{3+}+\frac{2 \cdot \pi}{3}\right)}+\frac{S_{-}}{3} \cdot e^{j\left(-3 \omega t+\phi^{3-}-\frac{2 \cdot \pi}{3}\right)}+\right. \\
\frac{S_{+}}{3} \cdot e^{j\left(3 \omega t+\phi^{3+}-3 \cdot 2 \cdot \frac{\pi}{3}\right)}+\frac{S_{-}}{3} \cdot e^{j\left(-3 \omega t+\phi^{-}-3 \cdot 2 \cdot \frac{\pi}{3}\right)}- \\
\left.\frac{S_{+}}{3} \cdot e^{j\left(3 \omega t+\phi^{3+}-\frac{2 \cdot \pi}{3}-3 \cdot \frac{\pi}{3}\right)}-\frac{S_{-}}{3} \cdot e^{j\left(-3 \omega t+\phi^{3-}+\frac{2 \cdot \pi}{3}-3 \cdot \frac{\pi}{3}\right)}\right)=0\end{array}$ \\
\hline Sinal Original $-5^{\circ}$ Ordem & Sinal extraído \\
\hline $\begin{array}{l}S_{r 5}(t)=\operatorname{Re}\left(S_{+} \cdot e^{j\left(5 \omega t+\phi^{5+}\right)}+\right. \\
\left.S_{-} . e^{j\left(-5 \omega t+\phi^{5-}\right)}\right)\end{array}$ & $\begin{array}{l}S_{r-}(t)=\operatorname{Re}\left(+\frac{S_{+}}{3} \cdot e^{j\left(5 \omega t+\phi^{5+}\right)}+\frac{S_{-}}{3} \cdot e^{j\left(-5 \omega t+\phi^{5-}\right)}\right. \\
+\frac{S_{+}}{3} \cdot e^{j\left(5 \omega t+\phi^{5+}-\frac{2 \cdot \pi}{3}-5 \cdot 2 \frac{\pi}{3}\right)}+\frac{S_{-}}{3} \cdot e^{j\left(-5 \omega t+\phi^{5-}+\frac{2 \cdot \pi}{3}-5 \cdot 2 \frac{\pi}{3}\right)} \\
\left.-\frac{S_{+}}{3} \cdot e^{j\left(5 \omega t+\phi^{5+}+\frac{2 \cdot \pi}{3}-5 \cdot \frac{\pi}{3}\right)}-\frac{S_{-}}{3} \cdot e^{j\left(-5 \omega t+\phi^{5-}-\frac{2 \cdot \pi}{3}-5 \cdot \frac{\pi}{3}\right)}\right)= \\
\operatorname{Re}\left(+S_{+} \cdot e^{j\left(5 \omega t+\phi^{5+}\right)}\right)\end{array}$ \\
\hline
\end{tabular}




\begin{tabular}{|l|l|}
\hline$S_{s 5}(t)=\operatorname{Re}\left(S_{+} \cdot e^{j\left(5 \omega t+\phi^{5+}-\frac{2 \cdot \pi}{3}\right)}+\right.$ & $S_{s-}(t)=\operatorname{Re}\left(+\frac{S_{+}}{3} \cdot e^{j\left(5 \omega t+\phi^{5+}-\frac{2 \cdot \pi}{3}\right)}+\frac{S_{-}}{3} \cdot e^{j\left(-5 \omega t+\phi^{5-}+\frac{2 \cdot \pi}{3}\right)}+\right.$ \\
$\left.S_{-} \cdot e^{j\left(-5 \omega t+\phi^{5-}+\frac{2 \cdot \pi}{3}\right)}\right)$ & $\frac{S_{+}}{3} \cdot e^{j\left(5 \omega t+\phi^{5+}+\frac{2 \cdot \pi}{3}-5 \cdot 2 \cdot \frac{\pi}{3}\right)}+\frac{S_{-}}{3} \cdot e^{j\left(-5 \omega t+\phi^{5-}-\frac{2 \cdot \pi}{3}-5 \cdot 2 \cdot \frac{\pi}{3}\right)}-$ \\
& $\left.\frac{S_{+}}{3} \cdot e^{j\left(5 \omega t+\phi^{5+}-5 \cdot \frac{\pi}{3}\right)}-\frac{S_{-}}{3} \cdot e^{j\left(-5 \omega t+\phi^{5-}-5 \cdot \frac{\pi}{3}\right)}\right)=$ \\
& $\operatorname{Re}\left(+S_{+} \cdot e^{j\left(5 \omega t+\phi^{5+}-\frac{2 \cdot \pi}{3}\right)}\right)$ \\
$S_{t 5}(t)=\operatorname{Re}\left(S_{+} \cdot e^{j\left(5 \omega t+\phi^{5+}+\frac{2 \cdot \pi}{3}\right)}+\right.$ & $S_{t-}(t)=\operatorname{Re}\left(+\frac{S_{+}}{3} \cdot e^{j\left(5 \omega t+\phi^{5+}+\frac{2 \cdot \pi}{3}\right)}+\frac{S_{-}}{3} \cdot e^{j\left(-5 \omega t+\phi^{5-}-\frac{2 \cdot \pi}{3}\right)}+\right.$ \\
$\left.S_{-} \cdot e^{j\left(-5 \omega t+\phi^{5-}-\frac{2 \cdot \pi}{3}\right)}\right)$ & $\frac{S_{+}}{3} \cdot e^{j\left(5 \omega t+\phi^{5+}-5 \cdot 2 \cdot \frac{\pi}{3}\right)}+\frac{S_{-}}{3} \cdot e^{j\left(-5 \omega t+\phi^{-}-5 \cdot 2 \cdot \frac{\pi}{3}\right)}-$ \\
& $\left.\frac{S_{+}}{3} \cdot e^{j\left(5 \omega t+\phi^{5+}-\frac{2 \cdot \pi}{3}-5 \cdot \frac{\pi}{3}\right)}-\frac{S_{-}}{3} \cdot e^{j\left(-5 \omega t+\phi^{5-}+\frac{2 \cdot \pi}{3}-5 \cdot \frac{\pi}{3}\right)}\right)=$ \\
& $\operatorname{Re}\left(+S_{+} \cdot e^{j\left(5 \omega t+\phi^{5+}+\frac{2 \cdot \pi}{3}\right)}\right)$ \\
\end{tabular}

\subsubsection{DEMONSTRAÇÃO MATEMÁTICA DA OPERAÇÃO “B” APLICADA A UM SINAL COM CONTEÚDO HARMÔNICO}

Hipótese a ser provada: a eq.(3.7) é válida para o domínio do tempo, em regime permanente para um sinal contendo harmônicos.

A seguir descreve-se o cálculo de $s_{r-}(t)$ (eq.(3.3.2-1)) substituindo os valores dados pelas eqs. (3.3-1), (3.3-2), (3.3-3) na eq.(3.7).

$$
\begin{aligned}
& S_{r-}(t)=\operatorname{Re}(\underbrace{\frac{S_{+}}{3} \cdot e^{j\left(h \omega t+\phi^{h+}\right)}}_{\text {termo } 1}+\underbrace{\frac{S_{-}}{3} \cdot e^{j\left(-h \omega t+\phi^{h-}\right)}}_{\text {termo } 2} \underbrace{-\frac{S_{+}}{6} \cdot e^{j\left(h \omega t+\phi^{h+}-\frac{2 \cdot \pi}{3}\right)}}_{\text {termo } 3}- \\
& \underbrace{\frac{S_{-}}{6} \cdot e^{j\left(-h \omega t+\phi^{h-}+\frac{2 . \pi}{3}\right)}}_{\text {termo } 4}-\underbrace{-\frac{S_{+}}{6} \cdot e^{j\left(h \omega t+\phi^{h+}+\frac{2 \cdot \pi}{3}\right)}}_{\text {termo } 5}-\underbrace{-\frac{S_{-}}{6} \cdot e^{j\left(-h \omega t+\phi^{h-}-\frac{2 \cdot \pi}{3}\right)}}_{\text {termo } 6}+ \\
& \underbrace{\frac{\sqrt{3}}{6} \cdot S_{+} \cdot e^{j\left(h \omega t+\phi^{h+}-\frac{2 \cdot \pi}{3}-h \cdot \frac{\pi}{2}\right)}}_{\text {termo } 7} \underbrace{+\frac{\sqrt{3}}{6} \cdot S_{-} \cdot e^{j\left(-h \omega t+\phi^{h-}+\frac{2 \cdot \pi}{3}-h \cdot \frac{\pi}{2}\right)}}_{\text {termo } 8}- \\
& \underbrace{\frac{\sqrt{3}}{6} \cdot S_{+} \cdot e^{j\left(h \omega t+\phi^{h+}+\frac{2 \cdot \pi}{3}-h \cdot \frac{\pi}{2}\right)}}_{\text {termo } 9}-\underbrace{\left.\left.-\frac{\sqrt{3}}{6} \cdot S_{-} \cdot e^{j\left(-h \omega t+\phi^{h-}-\frac{2 \cdot \pi}{3}-h \cdot \frac{\pi}{2}\right)}\right)\right)}_{\text {termo } 10}
\end{aligned}
$$


Utilizando-se a identidade $\cos (\alpha)=\frac{\mathrm{e}^{\mathrm{j} . \alpha}+\mathrm{e}^{-\mathrm{j} . \alpha}}{2}$ para os termos (3) e (5) da eq.(3.3.21), bem como para os termos (4) e (6), obtêm-se as equações (3.3.2-2) e (3.3.2-3).

$$
\begin{aligned}
& \text { Termo } 3+\text { termo } 5=-\frac{S_{+}}{6} \cdot e^{j\left(h \omega t+\phi^{h+}-\frac{2 \cdot \pi}{3}\right)}-\frac{S_{+}}{6} \cdot e^{j\left(h \omega t+\phi^{h+}+\frac{2 \cdot \pi}{3}\right)}= \\
& =-\frac{S_{+}}{6} \cdot e^{j\left(h \omega t+\phi^{+}\right)} \cdot 2 \cdot \cos \left(\frac{2 \cdot \pi}{3}\right)=\frac{S_{+}}{6} \cdot e^{j\left(h \omega t+\phi^{+}\right)} \\
& \text {Termo } 4+\text { termo } 6=-\frac{S_{-}}{6} \cdot e^{j\left(-h \omega t+\phi^{h-}+\frac{2 \cdot \pi}{3}\right)}-\frac{S_{-}}{6} \cdot e^{j\left(-h \omega t+\phi^{h-}-\frac{2 \cdot \pi}{3}\right)}= \\
& =-\frac{S_{-}}{6} \cdot e^{j\left(-h \omega t+\phi^{h-}\right)} \cdot 2 \cdot \cos \left(\frac{2 \cdot \pi}{3}\right)=+\frac{S_{-}}{6} \cdot e^{j\left(-h \omega t+\phi^{h-}\right)}
\end{aligned}
$$

Utilizando-se a identidade $\sin (\alpha)=\frac{\mathrm{e}^{\mathrm{j} . \alpha}-\mathrm{e}^{-\mathrm{j} . \alpha}}{2 . j}$ para os termos (7) e (9) da eq.(3.3.21), bem como para os termos (8) e (10), obtêm-se as equações (3.3.2-4) e (3.3.2-5).

$$
\begin{aligned}
& \text { Termo } 7+\text { Termo } 9=+\frac{\sqrt{3}}{6} \cdot S_{+} \cdot e^{j\left(h \omega t+\phi^{h+}-\frac{2 \cdot \pi}{3}-h \cdot \frac{\pi}{2}\right)}-\frac{\sqrt{3}}{6} \cdot S_{+} \cdot e^{j\left(h \omega t+\phi^{h+}+\frac{2 \cdot \pi}{3}-h \cdot \frac{\pi}{2}\right)}= \\
& -\frac{\sqrt{3}}{6} \cdot S_{+} \cdot e^{j\left(h \omega t+\phi^{h+}-h \cdot \frac{\pi}{2}\right)} \cdot 2 \cdot j \cdot \sin \left(+\frac{2 \cdot \pi}{3}\right)= \\
& -\frac{S_{+}}{2} \cdot e^{j\left(h \omega t+\phi^{h+}-h \cdot \frac{\pi}{2}\right)} \cdot e^{j\left(+\frac{\pi}{2}\right)}
\end{aligned}
$$

Termo $8+T$ ermo $10=-\frac{\sqrt{3}}{6} \cdot S_{-} \cdot e^{j\left(-h \omega t+\phi^{h-}+\frac{2 \cdot \pi}{3}-h \cdot \frac{\pi}{2}\right)}+\frac{\sqrt{3}}{6} \cdot S_{-} \cdot e^{j\left(-h \omega t+\phi^{h-}-\frac{2 \cdot \pi}{3}-h \cdot \frac{\pi}{2}\right)}=$ $-\frac{\sqrt{3}}{6} \cdot S_{-} \cdot e^{j\left(-h \omega t+\phi^{h-}-h \cdot \frac{\pi}{2}\right)} \cdot 2 \cdot j \cdot \sin \left(\frac{-2 \cdot \pi}{3}\right)=+\frac{1}{2} \cdot S_{-} . e^{j\left(-h \omega t+\phi^{h-}-h \cdot \frac{\pi}{2}\right)} \cdot e^{j\left(+\frac{\pi}{2}\right)}$

Substituindo-se os resultados das eqs.(3.3.2-2), (3.3.2-3), (3.3.2-4) e (3.3.2-5) na eq. (3.3.2-1) obtém-se o sinal instantâneo extraído da fase r na eq.(3.3.2-6).

$$
S_{r-}(t)=\operatorname{Re}\left(+\frac{S_{+}}{2} \cdot e^{j\left(h \omega t+\phi^{h+}\right)}+\frac{S_{-}}{2} \cdot e^{j\left(-h \omega t+\phi^{h-}\right)}-\frac{S_{+}}{2} \cdot e^{j\left(h \omega t+\phi^{h+}+\frac{\pi}{2} \cdot(1-h)\right)}+\frac{S_{-}}{2} \cdot e^{j\left(-h \omega t+\phi^{h-}+\frac{\pi}{2} \cdot(1-h)\right)}\right)
$$


Os sinais $\mathrm{s}_{\mathrm{s}-}(\mathrm{t})$ e $\mathrm{s}_{\mathrm{t}-}(\mathrm{t})$ seguem o mesmo procedimento e são apresentadas nas eqs. (3.3.2-7) e (3.3.2-8).

$$
\begin{aligned}
& S_{s-}(t)=\operatorname{Re}\left(-\frac{S_{+}}{2} \cdot e^{j\left(h \omega t+\phi^{h+}+\frac{\pi}{3}\right)}-\frac{S_{-}}{2} \cdot e^{j\left(-h \omega t+\phi^{h-}-\frac{\pi}{3}\right)}-\frac{S_{+}}{2} \cdot e^{\left.j\left(h \omega t+\phi^{h+}-h \cdot \frac{\pi}{2}-\frac{\pi}{6}\right)\right)}-\frac{S_{-}}{2} \cdot e^{\left.j\left(-h \omega t+\phi^{h-}-h \cdot \frac{\pi}{2}+\frac{\pi}{6}\right)\right)}\right) \\
& S_{t-}(t)=\operatorname{Re}\left(-\frac{S_{+}}{2} \cdot e^{j\left(h \omega t+\phi^{h+}-\frac{\pi}{3}\right)}-\frac{S_{-}}{2} \cdot e^{j\left(-h \omega t+\phi^{h-}+\frac{\pi}{3}\right)}-\frac{S_{+}}{2} \cdot e^{\left.j\left(h \omega t+\phi^{h+}-h \cdot \frac{\pi}{2}-\frac{5 \pi}{6}\right)\right)}-\frac{S_{-}}{2} \cdot e^{\left.j\left(-h \omega t+\phi^{h-}-h \cdot \frac{\pi}{2}+\frac{5 \pi}{6}\right)\right)}\right)
\end{aligned}
$$

Apresenta-se a seguir um exemplo de cálculo dos sinais extraídos (Tabela VI) para harmônicos de $2^{\circ}, 3^{\circ}$ e $5^{\circ}$ ordem de seqüência positiva e negativa comprovando assim a hipótese inicial e validando a Tabela I e II.

Tabela VI - Quadro demonstrativo dos sinais extraídos - operação B (com harmônicos)

\begin{tabular}{|c|c|}
\hline Sinal Original $-2^{0}$ Ordem & Sinal extraído \\
\hline$S_{r 2}(t)=\operatorname{Re}\left(S_{+} \cdot e^{j\left(2 \omega t+\phi^{2+}\right.}\right)+$ & $S_{r-}(t)=\operatorname{Re}\left(+\frac{S_{+}}{2} \cdot e^{j\left(2 \omega t+\phi^{2+}\right)}+\frac{S_{-}}{2} \cdot e^{j\left(-2 \omega t+\phi^{2-}\right)}\right.$ \\
$\left.S_{-} \cdot e^{j\left(-2 \omega t+\phi^{2-}\right)}\right)$ & $\left.-\frac{S_{+}}{2} \cdot e^{j\left(2 \omega t+\phi^{2+}+\frac{\pi}{2} \cdot(1-2)\right)}+\frac{S_{-}}{2} \cdot e^{j\left(-2 \omega t+\phi^{2-}+\frac{\pi}{2} \cdot(1-2)\right)}\right)=$ \\
\hline$S_{s 2}(t)=\operatorname{Re}\left(S_{+} \cdot e^{j\left(2 \omega t+\phi^{2+}-\frac{2 \cdot \pi}{3}\right)}+\right.$ & $\operatorname{Re}\left(\frac{7 \cdot S_{+}}{10} \cdot e^{j\left(2 \omega t+\phi^{2+}+\frac{\pi}{4}\right)}+\frac{7 \cdot S_{-}}{10} \cdot e^{j\left(-2 \omega t+\phi^{2-}-\frac{\pi}{4}\right)}\right)$ \\
$\left.S_{-} \cdot e^{j\left(-2 \omega t+\phi^{2-}+\frac{2 \cdot \pi}{3}\right)}\right)$ & $S_{s-}(t)=\operatorname{Re}\left(-\frac{S_{+}}{2} \cdot e^{j\left(2 \omega t+\phi^{2+}+\frac{\pi}{3}\right)}-\frac{S_{-}}{2} \cdot e^{j\left(-2 \omega t+\phi^{2-}-\frac{\pi}{3}\right)}\right.$ \\
\hline$S_{t 2}(t)=\operatorname{Re}\left(S_{+} \cdot e^{j\left(2 \omega t+\phi^{2+}+\frac{2 \cdot \pi}{3}\right)}+\right.$ & $\left.-\frac{S_{+}}{2} \cdot e^{\left.j\left(2 \omega t+\phi^{2+}-2 \cdot \frac{\pi}{2}-\frac{\pi}{6}\right)\right)}-\frac{S_{-}}{2} \cdot e^{\left.j\left(-2 \omega t+\phi^{2-}-2 \cdot \frac{\pi}{2}+\frac{\pi}{6}\right)\right)}\right)=$ \\
$\left.S_{-} \cdot e^{j\left(-2 \omega t+\phi^{2-}-\frac{2 \cdot \pi}{3}\right)}\right)$ & $\operatorname{Re}\left(\frac{7 \cdot S_{+}}{10} \cdot e^{j\left(2 \omega t+\phi^{2+}-\frac{2 \cdot \pi}{3}+\frac{\pi}{4}\right)}+\frac{7 \cdot S_{-}}{10} \cdot e^{j\left(-2 \omega t+\phi^{2-}+\frac{2 \cdot \pi}{3}-\frac{\pi}{4}\right)}\right.$ \\
& $S_{t-}(t)=\operatorname{Re}\left(-\frac{S_{+}}{2} \cdot e^{j\left(2 \omega t+\phi^{2+}-\frac{\pi}{3}\right)}-\frac{S_{-}}{2} \cdot e^{j\left(-2 \omega t+\phi^{2-}+\frac{\pi}{3}\right)}\right.$ \\
& $\left.-\frac{S_{+}}{2} \cdot e^{\left.j\left(2 \omega t+\phi^{2+}-2 \cdot \frac{\pi}{2}-\frac{5 \pi}{6}\right)\right)}-\frac{S_{-}}{2} \cdot e^{\left.j\left(-2 \omega t+\phi^{2-}-2 \cdot \frac{\pi}{2}+\frac{5 \pi}{6}\right)\right)}\right)=$ \\
& $\operatorname{Re}\left(\frac{7 \cdot S_{+}}{10} \cdot e^{j\left(2 \omega t+\phi^{2+}+\frac{2 \cdot \pi}{3}+\frac{\pi}{4}\right)}+\frac{7 \cdot S_{-}}{10} \cdot e^{j\left(-2 \omega t+\phi^{2-}-\frac{2 \cdot \pi}{3}-\frac{\pi}{4}\right)}\right.$ \\
\hline
\end{tabular}




\begin{tabular}{|c|c|}
\hline Sinal Original $-3^{\circ}$ Ordem & Sinal extraído \\
\hline $\begin{array}{l}S_{r 3}(t)=\operatorname{Re}\left(S_{+} \cdot e^{j\left(3 \omega t+\phi^{3+}\right)}+\right. \\
\left.S_{-} . e^{j\left(-3 \omega t+\phi^{3-}\right)}\right)\end{array}$ & $\begin{array}{l}S_{r-}(t)=\operatorname{Re}\left(+\frac{S_{+}}{2} \cdot e^{j\left(3 \omega t+\phi^{3+}\right)}+\frac{S_{-}}{2} \cdot e^{j\left(-3 \omega t+\phi^{3-}\right)}\right. \\
\left.-\frac{S_{+}}{2} \cdot e^{j\left(3 \omega t+\phi^{3+}+\frac{\pi}{2} \cdot(1-3)\right)}+\frac{S_{-}}{2} \cdot e^{j\left(-3 \omega t+\phi^{3-}+\frac{\pi}{2} \cdot(1-3)\right)}\right)= \\
\operatorname{Re}\left(S_{+} \cdot e^{j\left(3 \omega t+\phi^{3+}\right)}\right)\end{array}$ \\
\hline $\begin{array}{l}S_{s 3}(t)=\operatorname{Re}\left(S_{+} \cdot e^{j\left(3 \omega t+\phi^{3+}-\frac{2 . \pi}{3}\right)}+\right. \\
\left.S_{-} \cdot e^{j\left(-3 \omega t+\phi^{3-}+\frac{2 . \pi}{3}\right)}\right)\end{array}$ & $\begin{array}{l}S_{s-}(t)=\operatorname{Re}\left(-\frac{S_{+}}{2} \cdot e^{j\left(3 \omega t+\phi^{3+}+\frac{\pi}{3}\right)}-\frac{S_{-}}{2} \cdot e^{j\left(-3 \omega t+\phi^{3-}-\frac{\pi}{3}\right)}\right. \\
\left.-\frac{S_{+}}{2} \cdot e^{\left.j\left(3 \omega t+\phi^{3+}-3 \cdot \frac{\pi}{2}-\frac{\pi}{6}\right)\right)}-\frac{S_{-}}{2} \cdot e^{\left.j\left(-3 \omega t+\phi^{3-}-3 \cdot \frac{\pi}{2}+\frac{\pi}{6}\right)\right)}\right)= \\
\operatorname{Re}\left(S_{+} \cdot e^{j\left(3 \omega t+\phi^{3+}-\frac{2 \cdot \pi}{3}\right)}\right)\end{array}$ \\
\hline $\begin{array}{l}S_{t 3}(t)=\operatorname{Re}\left(S_{+} \cdot e^{j\left(3 \omega t+\phi^{3+}+\frac{2 \cdot \pi}{3}\right)}+\right. \\
\left.S_{-} \cdot e^{j\left(-3 \omega t+\phi^{3-}-\frac{2 . \pi}{3}\right)}\right)\end{array}$ & $\begin{array}{l}S_{t-}(t)=\operatorname{Re}\left(-\frac{S_{+}}{2} \cdot e^{j\left(3 \omega t+\phi^{3+}-\frac{\pi}{3}\right)}-\frac{S_{-}}{2} \cdot e^{j\left(-3 \omega t+\phi^{3-}+\frac{\pi}{3}\right)}\right. \\
\left.-\frac{S_{+}}{2} \cdot e^{\left.j\left(3 \omega t+\phi^{3+}-3 \cdot \frac{\pi}{2}-\frac{5 \pi}{6}\right)\right)}-\frac{S_{-}}{2} \cdot e^{\left.j\left(-3 \omega t+\phi^{3-}-3 \cdot \frac{\pi}{2}+\frac{5 \pi}{6}\right)\right)}\right)= \\
\operatorname{Re}\left(S_{+} \cdot e^{j\left(3 \omega t+\phi^{3+}+\frac{2 \cdot \pi}{3}\right)}\right)\end{array}$ \\
\hline Sinal Original $-5^{\circ}$ Ordem & Sinal extraído \\
\hline $\begin{array}{l}S_{r 5}(t)=\operatorname{Re}\left(S_{+} \cdot e^{j\left(5 \omega t+\phi^{5+}\right)}+\right. \\
\left.S_{-} \cdot e^{j\left(-5 \omega t+\phi^{5-}\right)}\right)\end{array}$ & $\begin{array}{l}S_{r-}(t)=\operatorname{Re}\left(+\frac{S_{+}}{2} \cdot e^{j\left(5 \omega t+\phi^{5+}\right)}+\frac{S_{-}}{2} \cdot e^{j\left(-5 \omega t+\phi^{5-}\right)}\right. \\
\left.-\frac{S_{+}}{2} \cdot e^{j\left(5 \omega t+\phi^{5+}+\frac{\pi}{2} \cdot(1-5)\right)}+\frac{S_{-}}{2} \cdot e^{j\left(-5 \omega t+\phi^{5-}+\frac{\pi}{2} \cdot(1-5)\right)}\right)= \\
\operatorname{Re}\left(+S_{-} \cdot e^{j\left(-5 \omega t+\phi^{5-}\right)}\right)\end{array}$ \\
\hline $\begin{array}{l}S_{s 5}(t)=\operatorname{Re}\left(S_{+} \cdot e^{j\left(5 \omega t+\phi^{5+}-\frac{2 . \pi}{3}\right)}+\right. \\
\left.S_{-} \cdot e^{j\left(-5 \omega t+\phi^{5-}+\frac{2 . \pi}{3}\right)}\right)\end{array}$ & $\begin{array}{l}S_{S-}(t)=\operatorname{Re}\left(-\frac{S_{+}}{2} \cdot e^{j\left(5 \omega t+\phi^{5+}+\frac{\pi}{3}\right)}-\frac{S_{-}}{2} \cdot e^{j\left(-5 \omega t+\phi^{5-}-\frac{\pi}{3}\right)}\right. \\
\left.-\frac{S_{+}}{2} \cdot e^{\left.j\left(5 \omega t+\phi^{5+}-5 \cdot \frac{\pi}{2}-\frac{\pi}{6}\right)\right)}-\frac{S_{-}}{2} \cdot e^{\left.j\left(-5 \omega t+\phi^{5-}-5 \cdot \frac{\pi}{2}+\frac{\pi}{6}\right)\right)}\right)= \\
\operatorname{Re}\left(S_{-} \cdot e^{j\left(-5 \omega t+\phi^{5-}+\frac{2 \cdot \pi}{3}\right)}\right)\end{array}$ \\
\hline $\begin{array}{l}S_{t 5}(t)=\operatorname{Re}\left(S_{+} \cdot e^{j\left(5 \omega t+\phi^{5+}+\frac{2 \cdot \pi}{3}\right)}+\right. \\
\left.S_{-} \cdot e^{j\left(-5 \omega t+\phi^{5-}-\frac{2 . \pi}{3}\right)}\right)\end{array}$ & $\begin{array}{l}S_{t-}(t)=\operatorname{Re}\left(-\frac{S_{+}}{2} \cdot e^{j\left(5 \omega t+\phi^{5+}-\frac{\pi}{3}\right)}-\frac{S_{-}}{2} \cdot e^{j\left(-5 \omega t+\phi^{5-}+\frac{\pi}{3}\right)}\right. \\
\left.-\frac{S_{+}}{2} \cdot e^{\left.j\left(5 \omega t+\phi^{5+}-5 \cdot \frac{\pi}{2}-\frac{5 \pi}{6}\right)\right)}-\frac{S_{-}}{2} \cdot e^{\left.j\left(-5 \omega t+\phi^{5-}-5 \cdot \frac{\pi}{2}+\frac{5 \pi}{6}\right)\right)}\right)= \\
\operatorname{Re}\left(S_{-} \cdot e^{j\left(-5 \omega t+\phi^{5-}-\frac{2 \cdot \pi}{3}\right)}\right)\end{array}$ \\
\hline
\end{tabular}

De forma análoga, as demonstrações dos itens 3.3.1 e 3.3.2 valem respectivamente para as operações C e D.

Método de extração em tempo real de seqüência positiva, negativa e/ou harmônicos. 


\subsection{Estratégias para separar os distúrbios individualmente}

Uma análise detalhada da tabela I, mostra que é possível combinar as operações (A, B, C, D) para obter uma determinada estratégia de extração. A Fig.3a mostra como a seqüência positiva da fundamental pode ser extraída de um sinal distorcido e desequilibrado através da operação em cascata das operações C e D. De modo análogo, a Fig.3b mostra como a seqüência negativa da fundamental pode ser extraída através da operação em cascata das operações A e B. A Fig.3c mostra a extração das componentes harmônicas apresentando-se como possível aplicação em filtros de harmônicos. A Fig.3d mostra a extração das componentes harmônicas e desequilíbrios. Apenas as harmônicas ímpares são consideradas nas figuras (3a, $3 b, 3 c, 3 d)$.

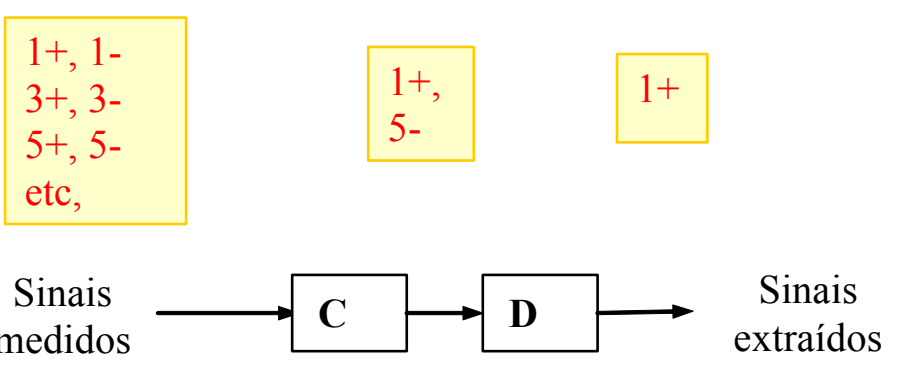

Fig. 3a - Diagrama em blocos do algoritmo de extração da componente de seqüência positiva da fundamental

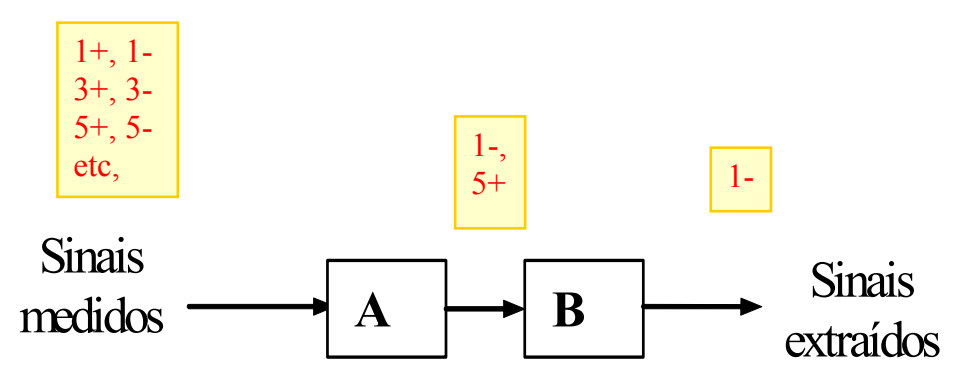

Fig. 3b - Diagrama em blocos do algoritmo de extração da componente de seqüência negativa da fundamental 


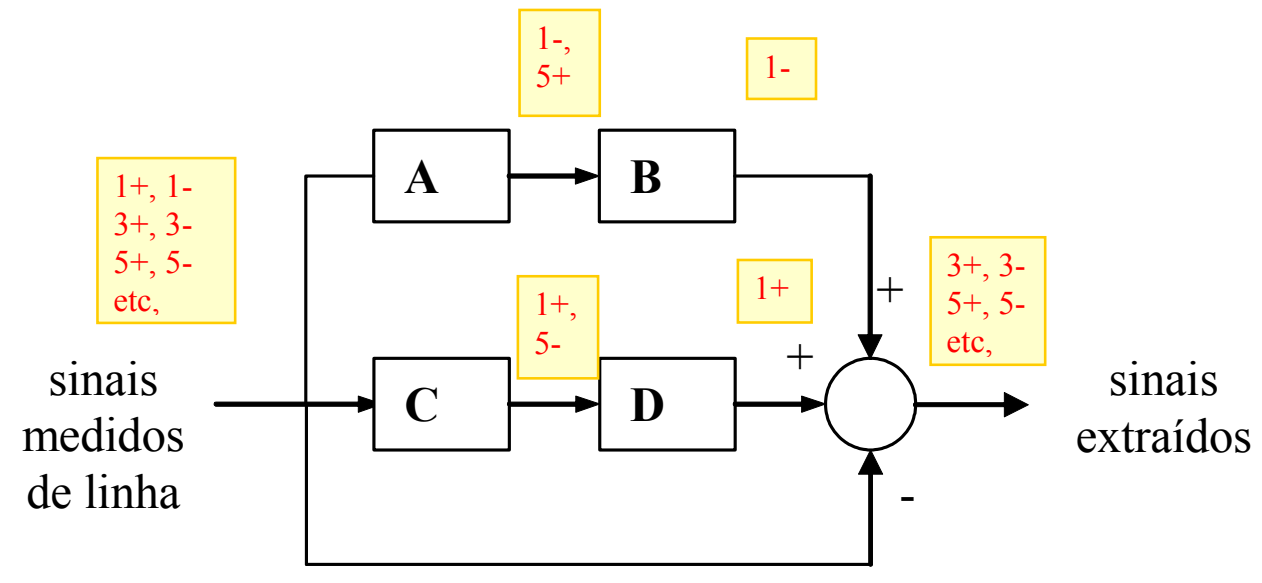

Fig. 3c - Diagrama em blocos do algoritmo de extração dos harmônicos

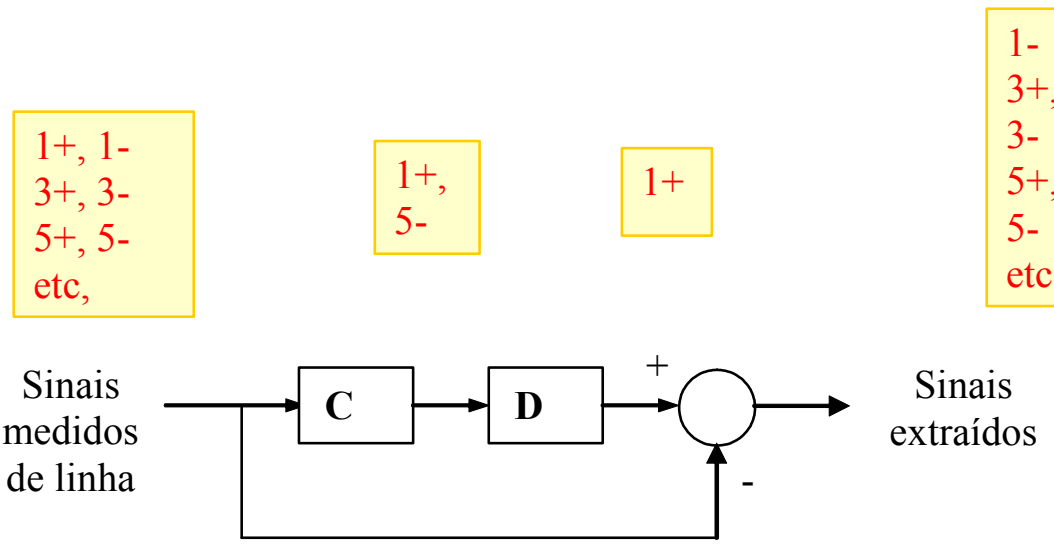

Fig. 3d - Diagrama em blocos do algoritmo de extração da componente de seqüência negativa e harmônicos (extração total)

A extração da seqüência zero no domínio do tempo e a influência dos métodos propostos no processamento de sinais com seqüência zero será apresentada no Apêndice $A$. 


\section{SIMULAÇÃO NUMÉRICA E IMPLEMENTAÇÃO EM DSP DO ALGORITMO DE EXTRAÇÃO PROPOSTO}

O método proposto no cap.3 para extração das componentes de seqüência positiva, negativa e/ou harmônicos é analisado a partir de resultados obtidos via simulação numérica e implementação digital em um Processador Digital de Sinais.

As operações (A, B, C, D, AB e CD) apresentadas no cap.3 foram simuladas numericamente e implementadas no DSP ADMC401 da Analog Devices, seus sinais foram adquiridos através do software Wavestar e processados no software MATLAB.

Tendo-se por base um sinal distorcido, contendo harmônicos e/ou desequilíbrios apresentam-se:

- a análise das respostas em regime e transitória das operações propostas;

- um comparativo entre os resultados teóricos, simulados no software MATLAB e os obtidos experimentalmente;

- a análise da influência da variação da freqüência dos sinais processados no desempenho dos métodos propostos;

- o tempo de execução de cada rotina.

Todas as amplitudes dos sinais são representadas, neste capítulo, pelos seus valores de pico, exceto quando especificado em contrário. 


\subsection{Resposta em Regime}

O comportamento das operações para o cálculo da componente de seqüência positiva, negativa e/ou harmônicos, vistos no capítulo 3, é analisado para um sinal distorcido.

A fim de permitir uma melhor análise das operações propostas, utilizam-se dois sinais distorcidos, sendo um composto exclusivamente por harmônicos impares (a) e outro por harmônicos pares (b).

O sinal distorcido composto por harmônicos impares (Fig.4.1.1a) tem a seguinte característica:

- 0.5 p.u. de seqüência positiva e 0.5 p.u. de seqüência negativa da fundamental com defasagem nula;

- 0.15 p.u. de seqüência positiva e 0.15 p.u. de seqüência negativa de terceira harmônica com defasagem nula;

- 0.15 p.u. de seqüência positiva e 0.15 p.u. de seqüência negativa de quinta harmônica com defasagem nula;

O sinal distorcido composto por harmônicos pares (Fig.4.1.1b) tem a seguinte característica:

- 0.4 p.u. de seqüência positiva e 0.4 p.u. de seqüência negativa da fundamental com defasagem nula;

- 0.15 p.u. de seqüência positiva e 0.15 p.u. de seqüência negativa de segunda harmônica com defasagem nula;

- 0.15 p.u. de seqüência positiva e 0.15 p.u. de seqüência negativa de quarta harmônica com defasagem nula;

Devido aos valores de amplitude e defasagem dos sinais distorcidos de teste, o sinal da fase $S$ (verde) se encontra em fase (sobreposto) com o sinal da Fase $T$ (azul). 
As figuras 4.1.2(a) e 4.1.2(b) apresentam os sinais gerados experimentalmente através de tabela pré-programada no DSP.

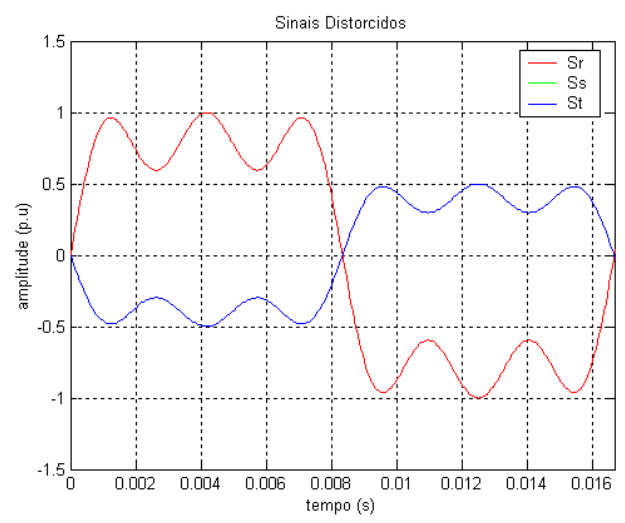

Fig.4.1.1a - Sinal distorcido simulado

$$
\left(1^{0}+-, 3^{\circ}+-, 5^{\circ}+-\right)
$$

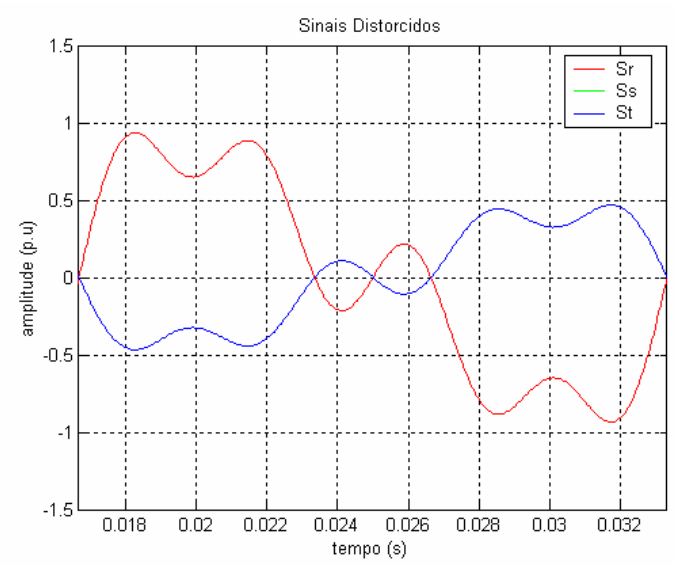

Fig.4.1.1b - Sinal distorcido simulado $\left(1^{0}+-, 2^{0}+-, 4^{0}+-\right)$

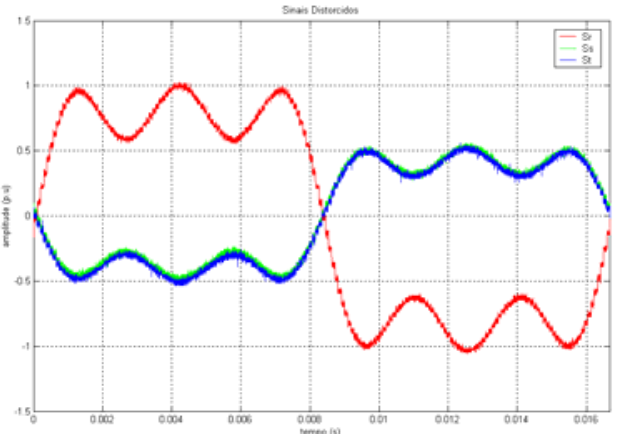

Fig.4.1.2a - Sinal distorcido experimentalmente gerado através de matriz de pontos pré-programada

$$
\left(1^{\circ+-}, 3^{\circ}+-, 5^{\circ}+-\right)
$$

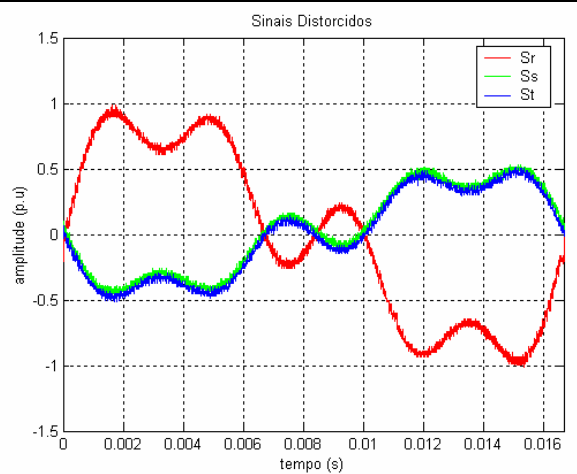

Fig.4.1.2b - Sinal distorcido experimentalmente gerado através de matriz de pontos pré-programada

$$
\left(1^{\circ}+-, 2^{\circ}+-, 4^{\circ}+-\right)
$$

Apresenta-se a seguir (Figs.4.1.3 à 4.1.14) as formas de onda (simulada e experimental) extraídas em cada operação (A, B, C, D, AB, CD), mantendo-se o índice (a) relativo ao sinal de teste que contem harmônicos impares e (b) para o que contem harmônicos pares. 


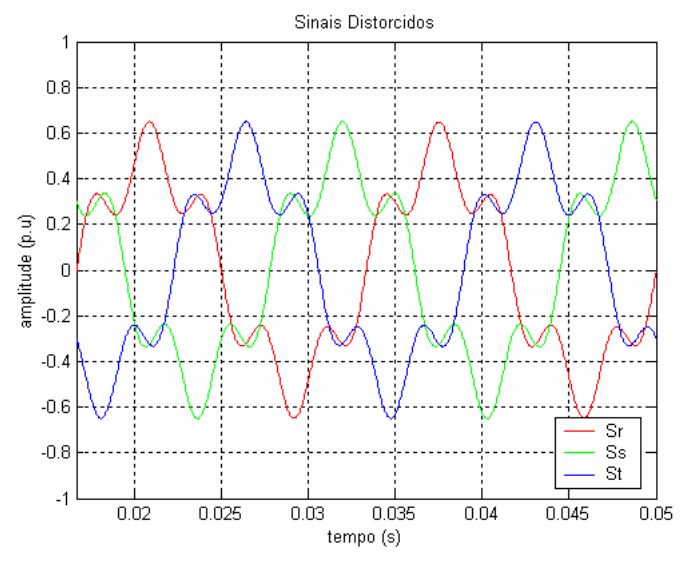

Fig.4.1.3a - Sinais extraídos Método A (Simulação) - $\left(1^{\circ}-, 5^{\circ}+\right)$

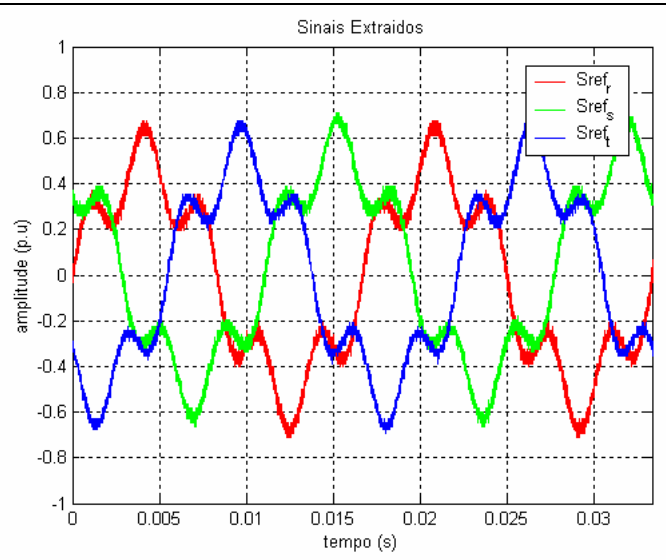

Fig.4.1.4a - Sinais extraídos Método $A$ (Experimental) - $\left(1^{\circ}-, 5^{\circ}+\right)$

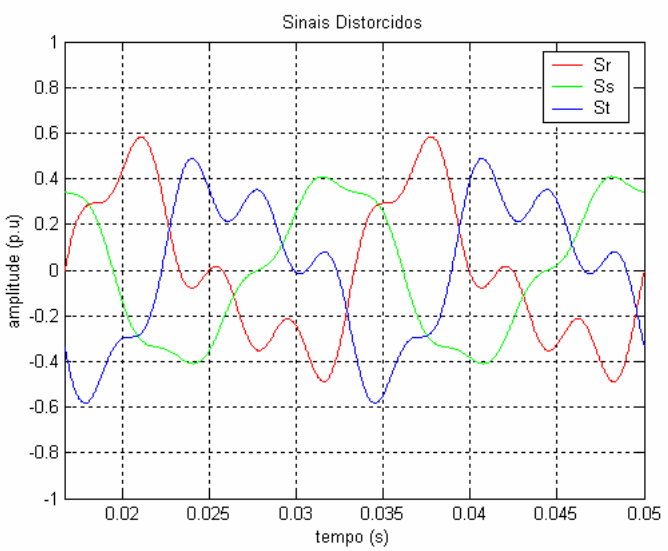

Fig.4.1.3b - Sinais extraídos Método A (Simulação) - $\left(1^{\circ}+-, 2^{\circ}+-, 4^{\circ}+-\right)$

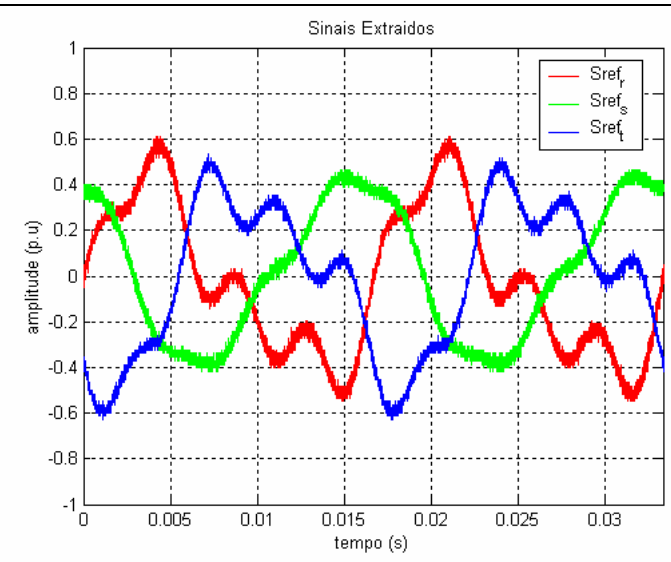

Fig.4.1.4b - Sinais extraídos Método A (Experimental) - $\left(1^{\circ}+-, 2^{0_{+-}}\right.$ , $\left.4^{\circ}+-\right)$ 


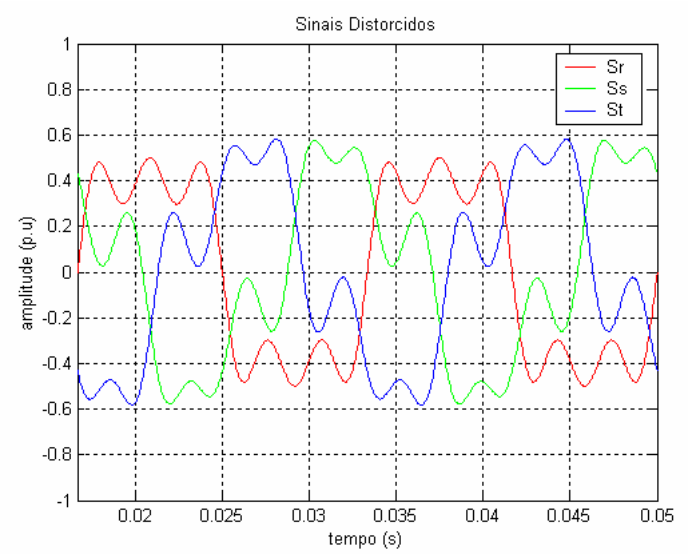

Fig.4.1.5a - Sinais extraídos Método B (Simulação) - $\left(1^{\circ}-, 3^{\circ}+, 5^{\circ}-\right)$

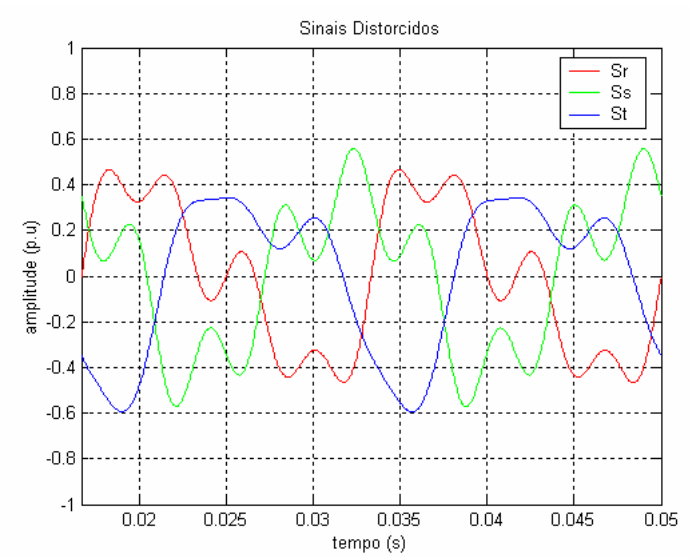

Fig.4.1.5b - Sinais extraídos - Método

B (Simulação) $-\left(1^{\circ}+-, 2^{\circ}+-, 4^{\circ}+-\right)$

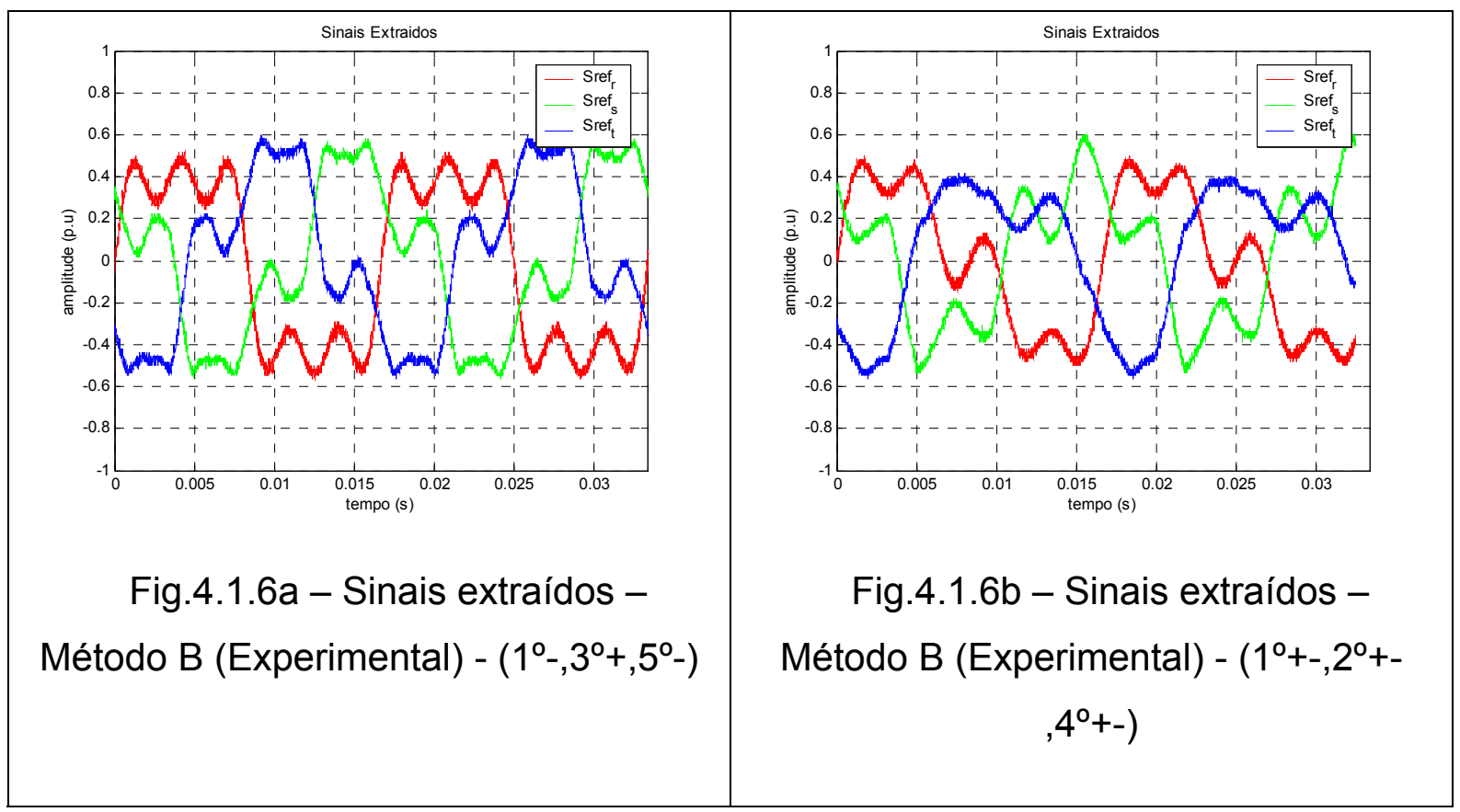




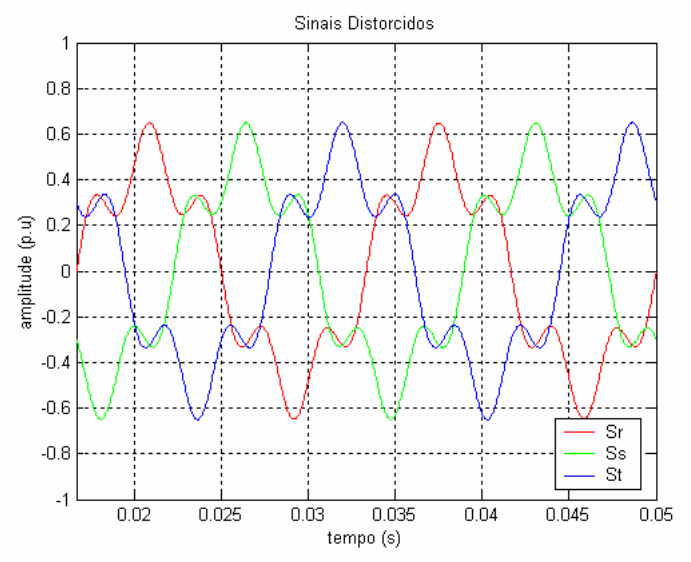

Fig.4.1.7a - Sinais extraídos Método C (Simulação) - $\left(1^{\circ}+, 5^{\circ}-\right)$

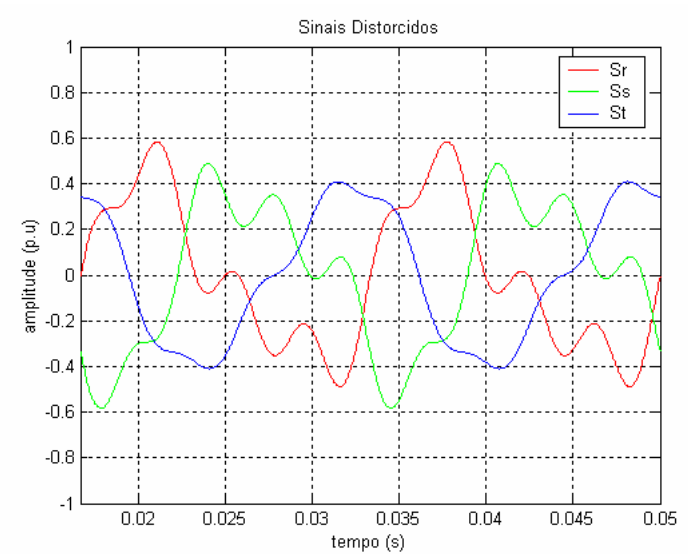

Fig.4.1.7b - Sinais extraídos Método C (Simulação) - $\left(1^{\circ}+-, 2^{\circ}+-, 4^{\circ}+-\right)$

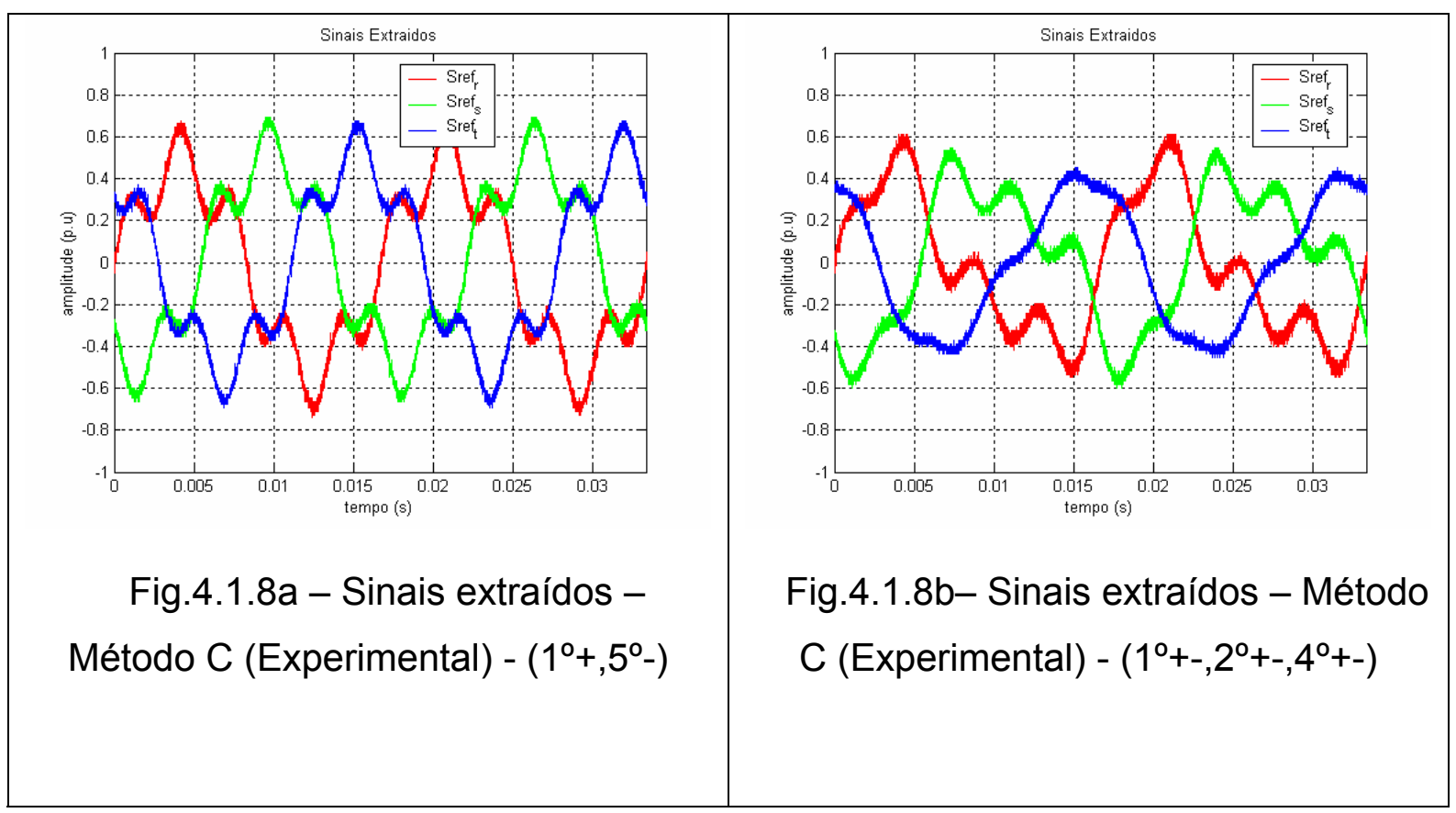




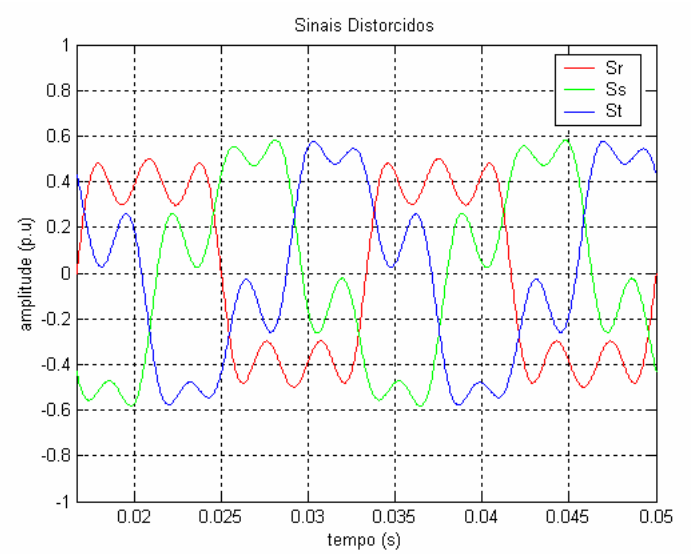

Fig.4.1.9a - Sinais extraídos Método D (Simulação) $-\left(1^{\circ}+, 3^{\circ}-, 5^{\circ}+\right)$

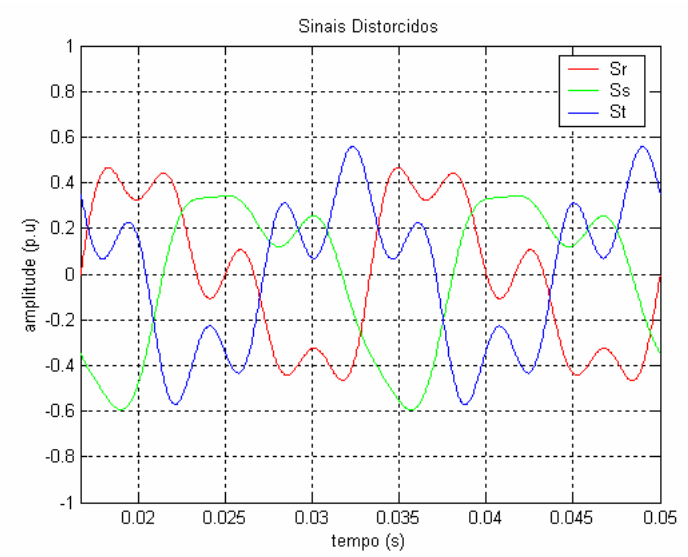

Fig.4.1.9b - Sinais extraídos Método D (Simulação) - $\left(1^{\circ}+-, 2^{\circ}+-, 4^{\circ}+-\right)$

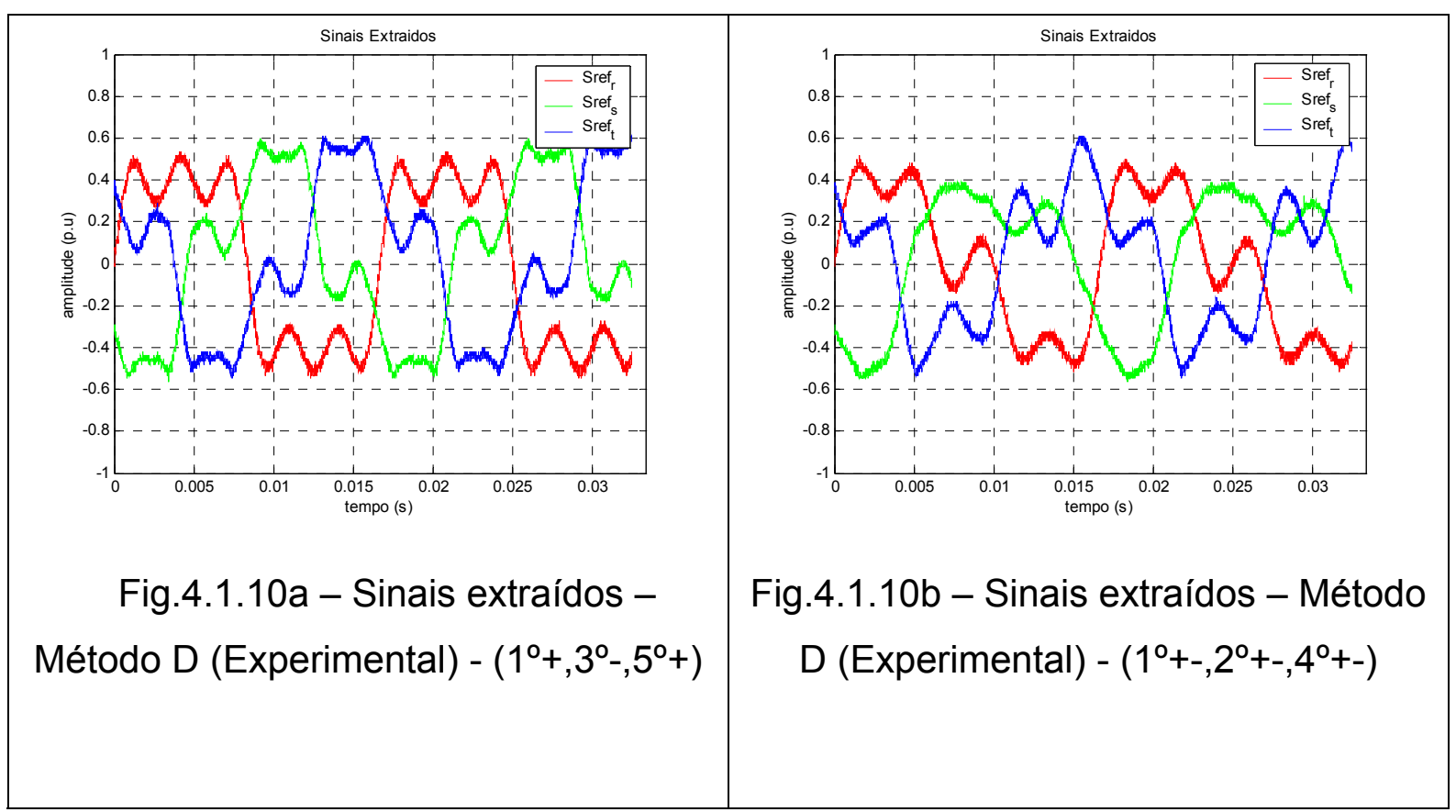




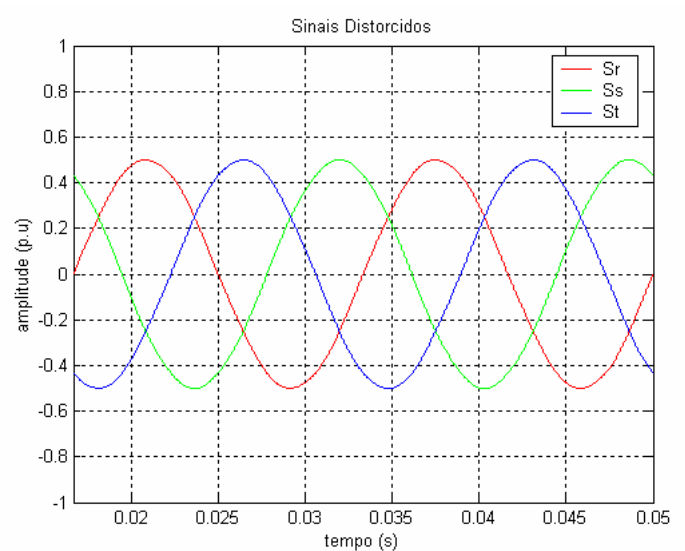

Fig.4.1.11a - Sinais extraídos Método AB (Simulação) - (10-)

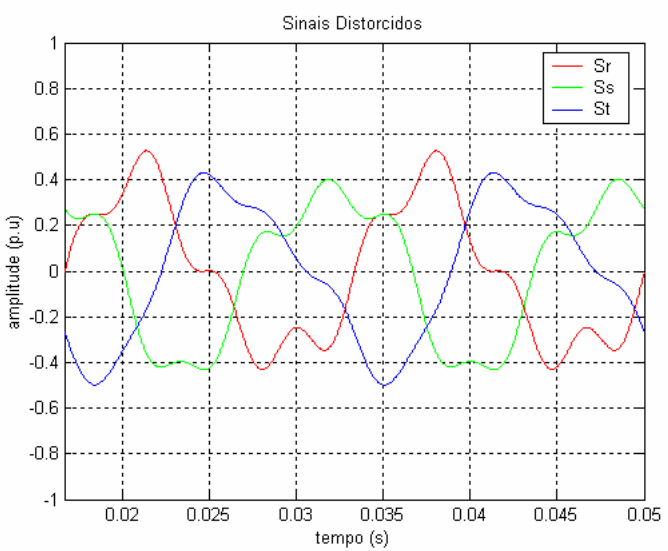

Fig.4.1.11b - Sinais extraídos Método AB (Simulação) - $\left(1^{\circ}+-, 2^{\circ}+-, 4^{\circ}+-\right.$

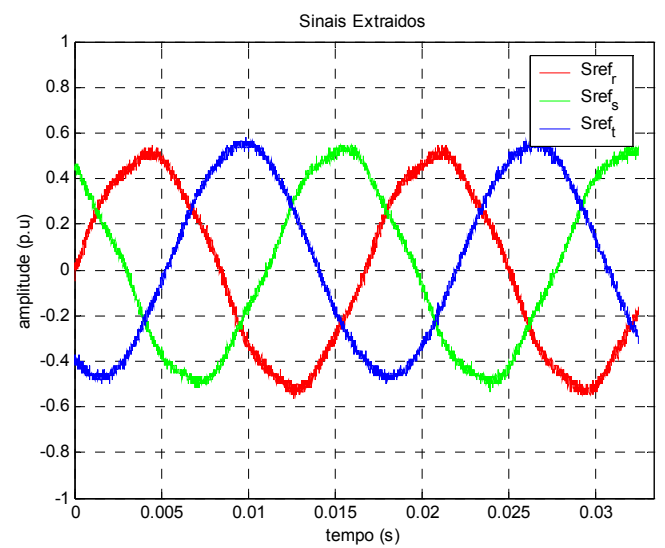

Fig.4.1.12a - Sinais extraídos - Método $A B$ (Experimental) $-\left(1^{\circ}-\right)$

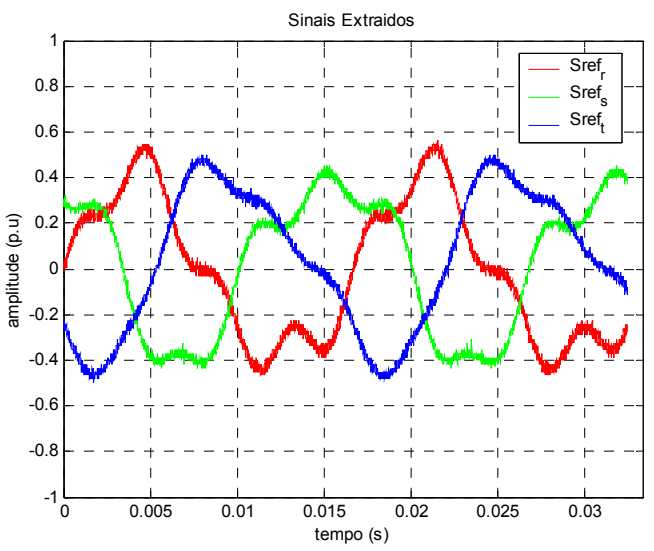

Fig.4.1.12b - Sinais extraídos - Método $A B($ Experimental $)-\left(1^{\circ}+-, 2^{\circ}+-, 4^{\circ}+-\right)$ 


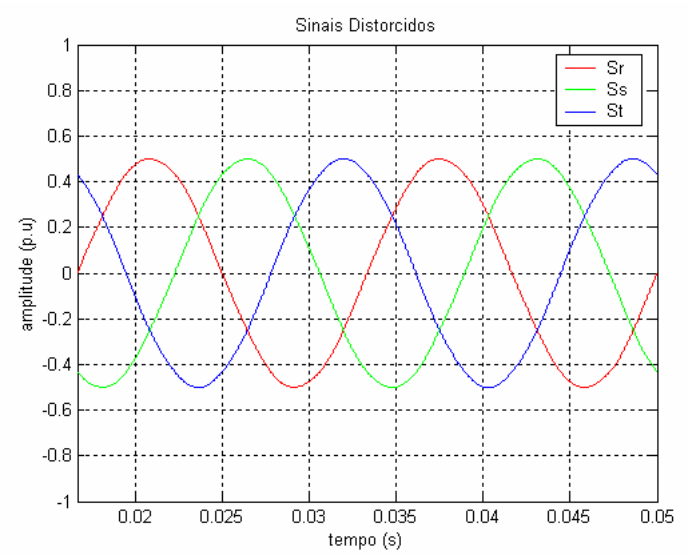

Fig.4.1.13a - Sinais extraídos Método CD (Simulação) - $\left(1^{\circ}+\right)$

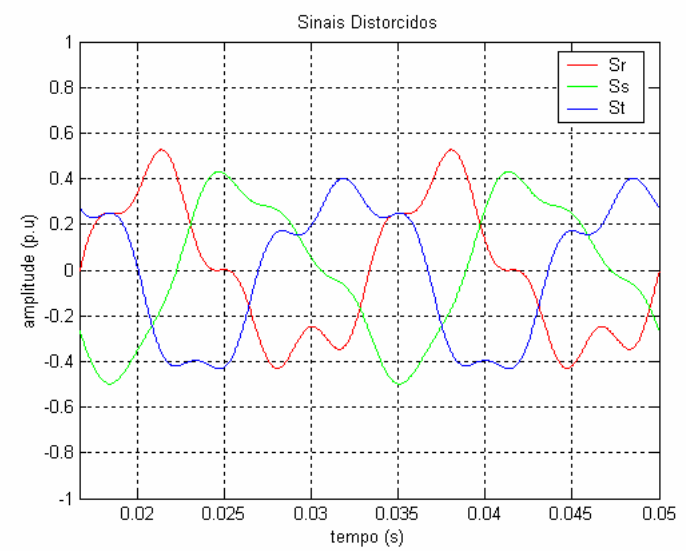

Fig.4.1.13b - Sinais extraídos Método CD (Simulação) $-\left(1^{\circ}+\right)$

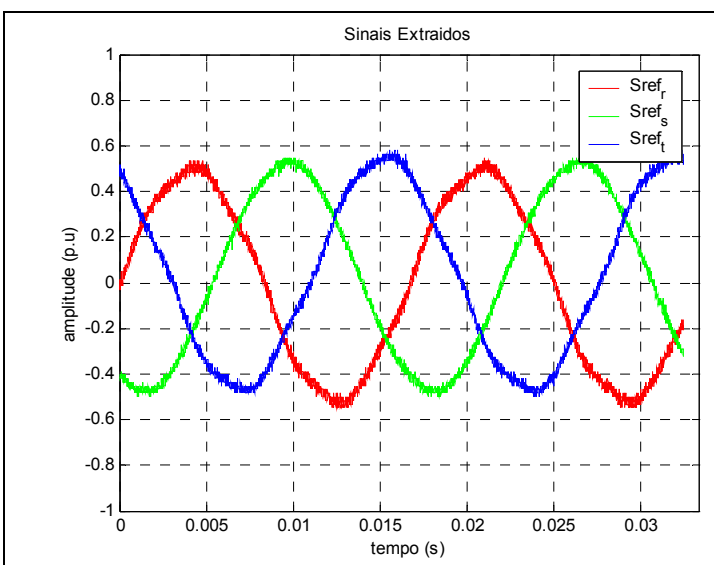

Fig.4.1.14a - Sinais extraídos Método CD (Experimental) - $\left(1^{0}+\right)$

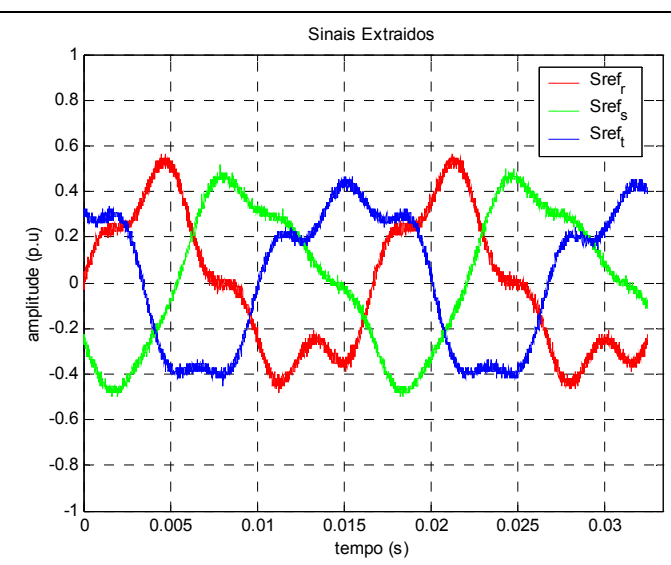

Fig.4.1.14b - Sinais extraídos Método CD (Experimental) - $\left(1^{\circ}+\right)$

As figuras 4.1.15a, 4.1.16a e 4.1.17a (harmônicos impares) e Figs.4.1.15b, 4.1.16b e 4.1.17b (harmônicos pares) mostram:

- a amplitude dos sinais de seqüências positiva e negativa calculados teoricamente utilizando-se os valores de ganhos apresentados nas tabelas I e Il do capitulo 3;

- as amplitudes, obtidas via simulação numérica e implementação experimental do algoritmo. As seqüências, positiva e negativa, dos sinais extraídos são obtidas através de pós-processamento numérico através de rotina implementada no programa MATLAB. 


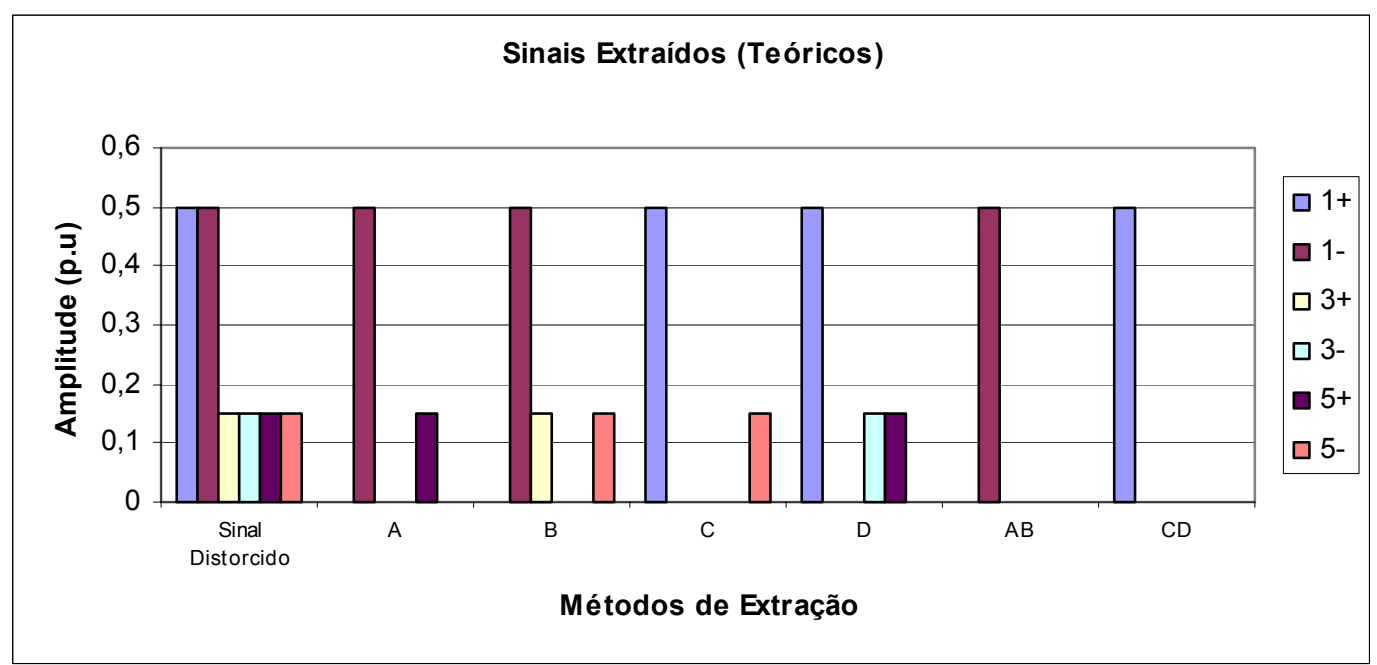

Fig.4.1.15a - Seqüências dos sinais extraídos - (Teórico - tabela I cap.3)

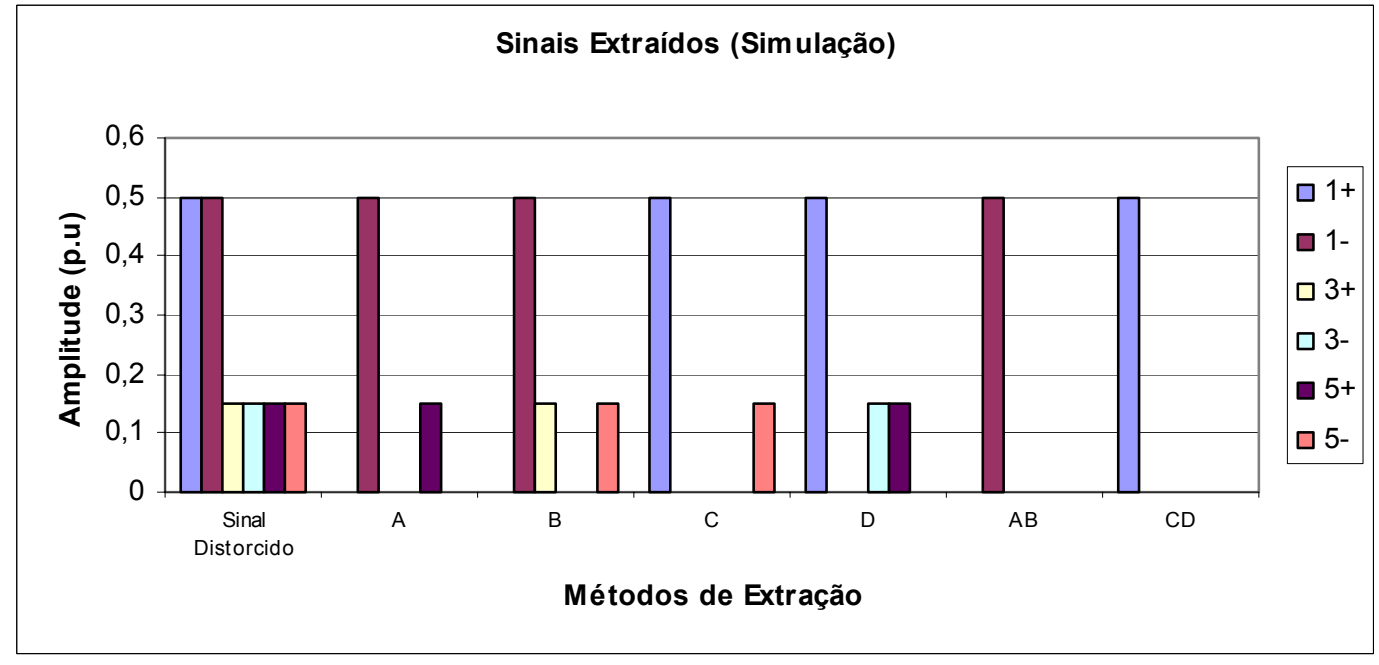

Fig.4.1.16a - Seqüências dos sinais extraídos - (Simulado)

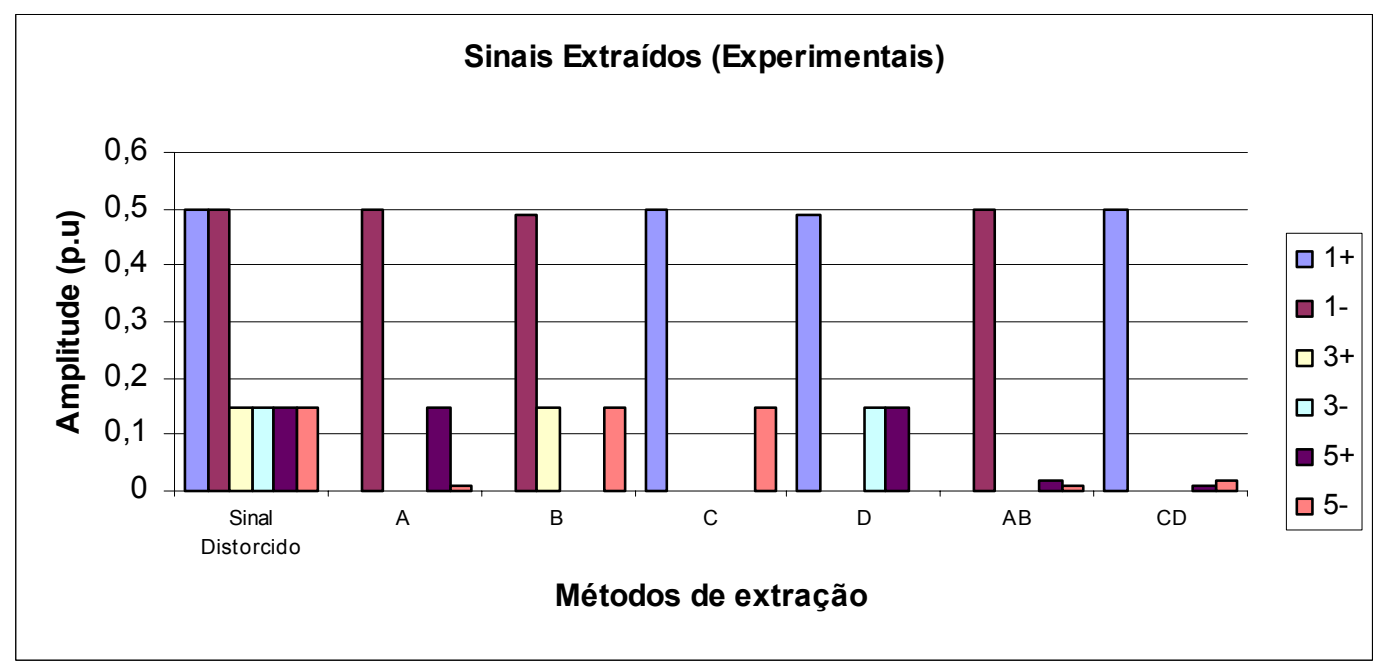

Fig.4.1.17a - Seqüências dos sinais extraídos (Experimental) 


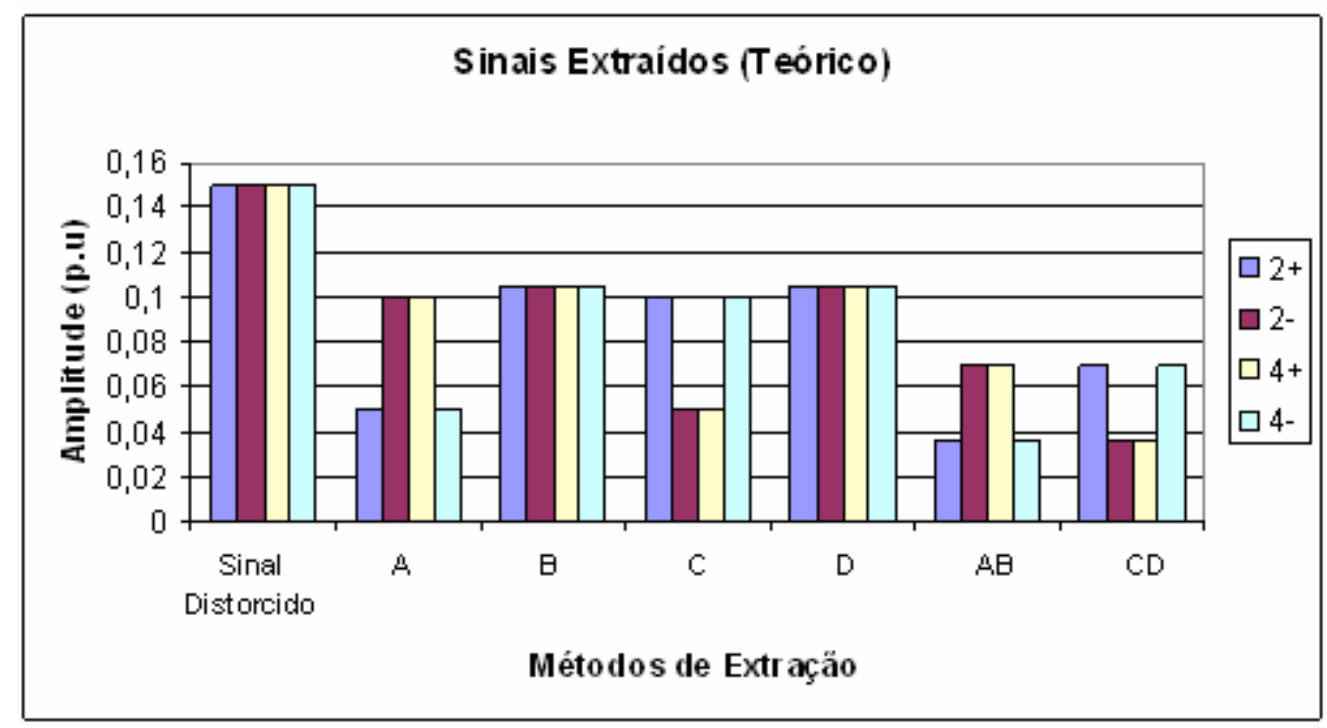

Fig.4.1.15b - Seqüências dos sinais extraídos - (Teórico - tabela II, cap.3)

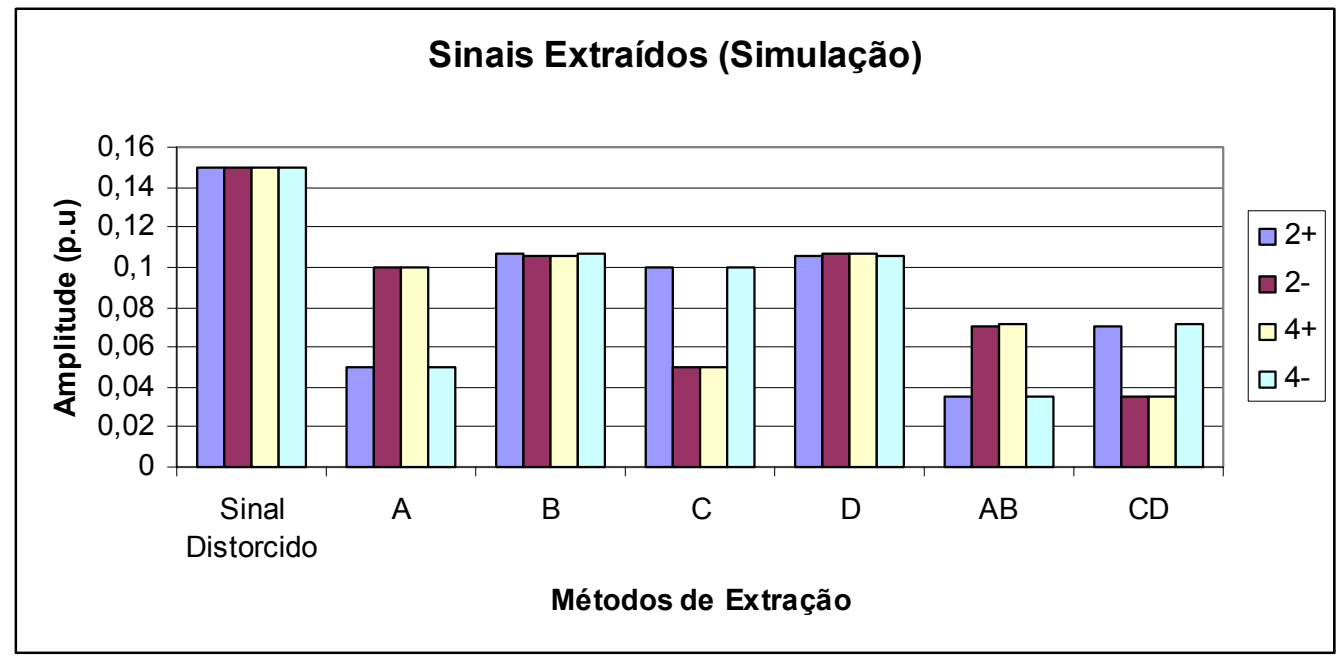

Fig.4.1.16b - Seqüências dos sinais extraídos - (Simulado)

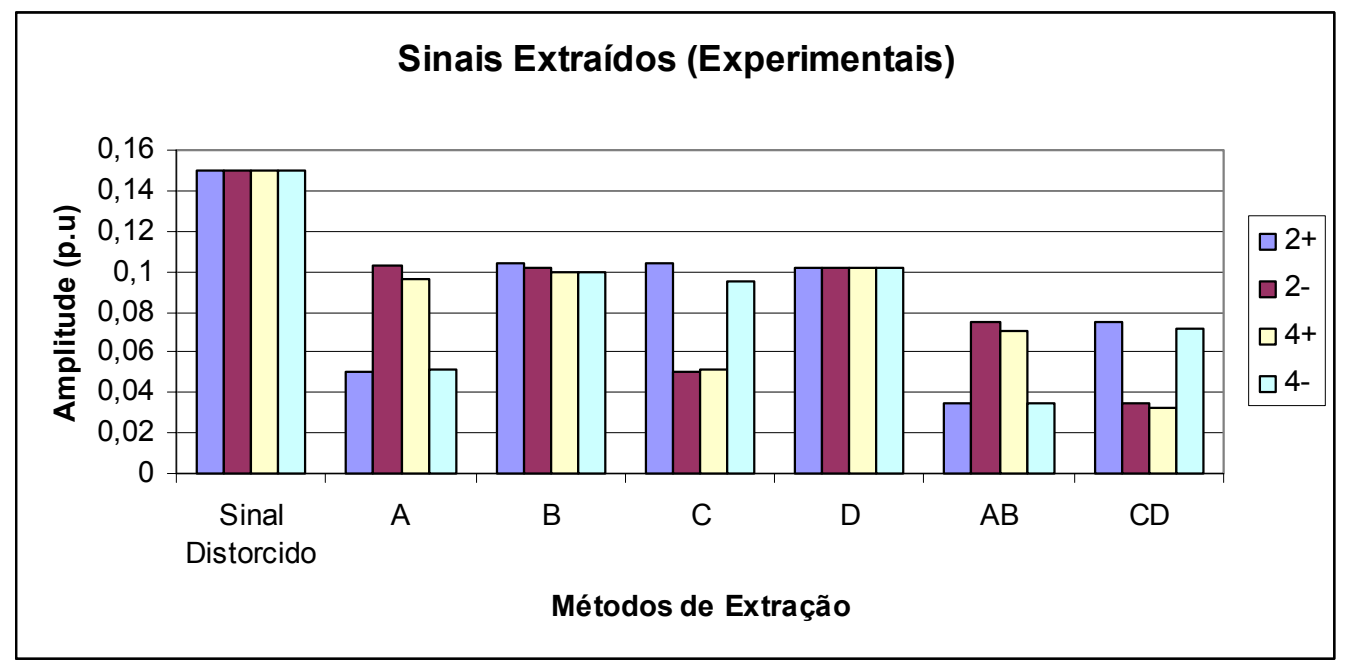

Fig.4.1.17b - Seqüências dos sinais extraídos (Experimental) 
Observa-se pelas Figs.4.1.15, 4.1.16 e 4.1.17 que o valor das seqüências (positiva, negativa) calculados teoricamente, obtidos via simulação e experimentalmente são muito próximos mas não completamente iguais, ou seja, não houve alteração significativa nos valores previstos inicialmente, o que demonstra a validade dos métodos propostos, no entanto, há necessidade de melhor ajuste do algoritmo implementado no DSP de modo a diminuir distorções encontradas possivelmente por estouro de overflow nas etapas intermediárias de cálculo.

\subsection{Resposta Transitória}

O comportamento transitório das operações vistas no capítulo 3 é analisado para um sinal contendo transições em degrau.

O sinal é composto por:

- um ciclo com amplitude nula (0 a $1 / 60$ s)

- dois ciclos contendo 0.25 p.u. de seqüência positiva e 0.25 p.u. de seqüência negativa da fundamental com defasagem $180^{\circ}$ (graus), ou seja, emulando uma carga monofásica ligada apenas entre as fases S e T (1/60 a 3/60 s);

- um ciclo com amplitude nula (3/60 a 4/60 s);

Na figura 4.2.1 é apresentado o terno de sinais desequilibrados gerado para teste da resposta transitória. Todos os sinais deste item (4.2) terão o índice (a) quando obtidos via simulação e o índice (b) quando obtidos experimentalmente.

O sinal de teste experimental foi obtido através de tabela pré-programada no DSP. 


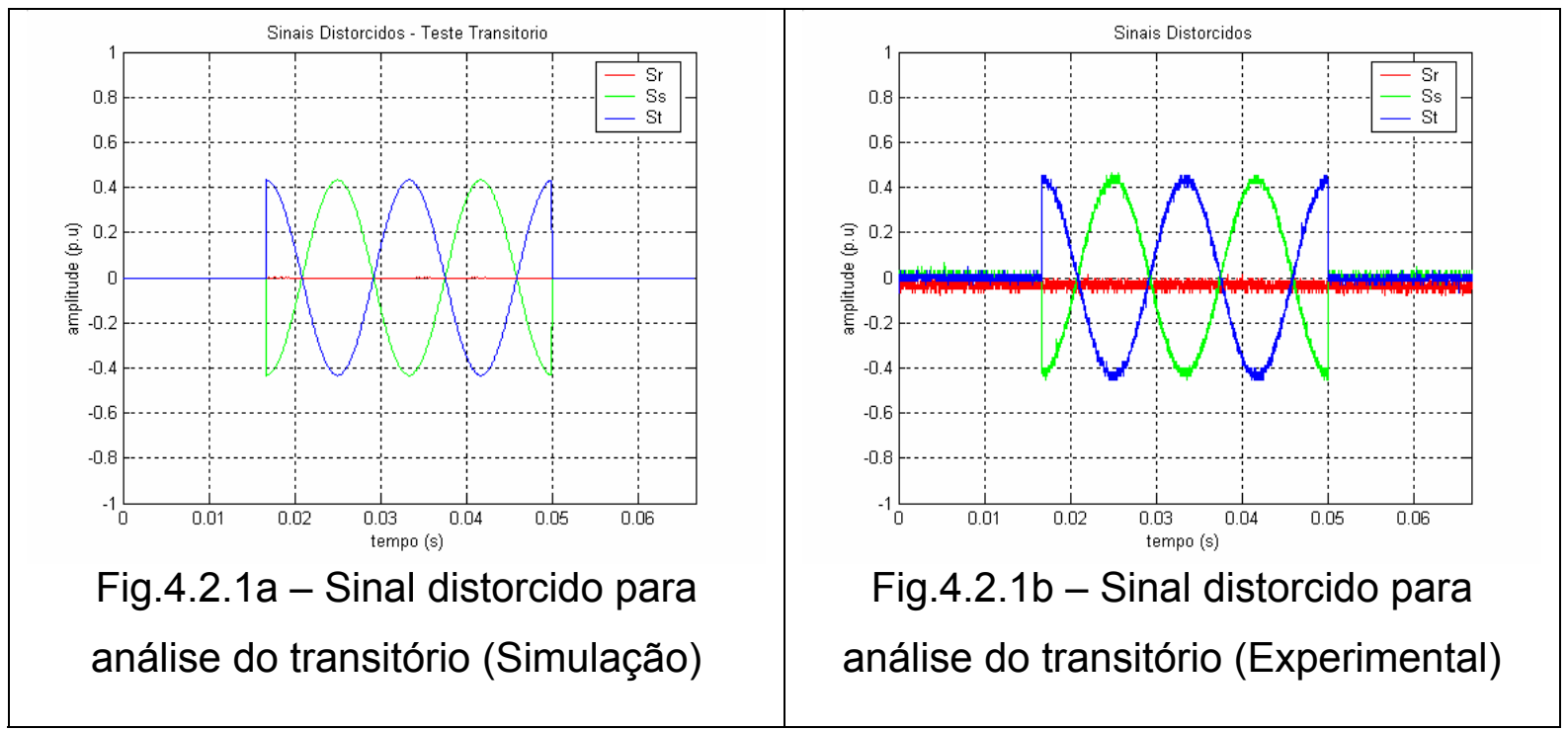

Apresenta-se a seguir as formas de onda extraídas em cada operação (Figs.4.2.2 à 4.2.7).

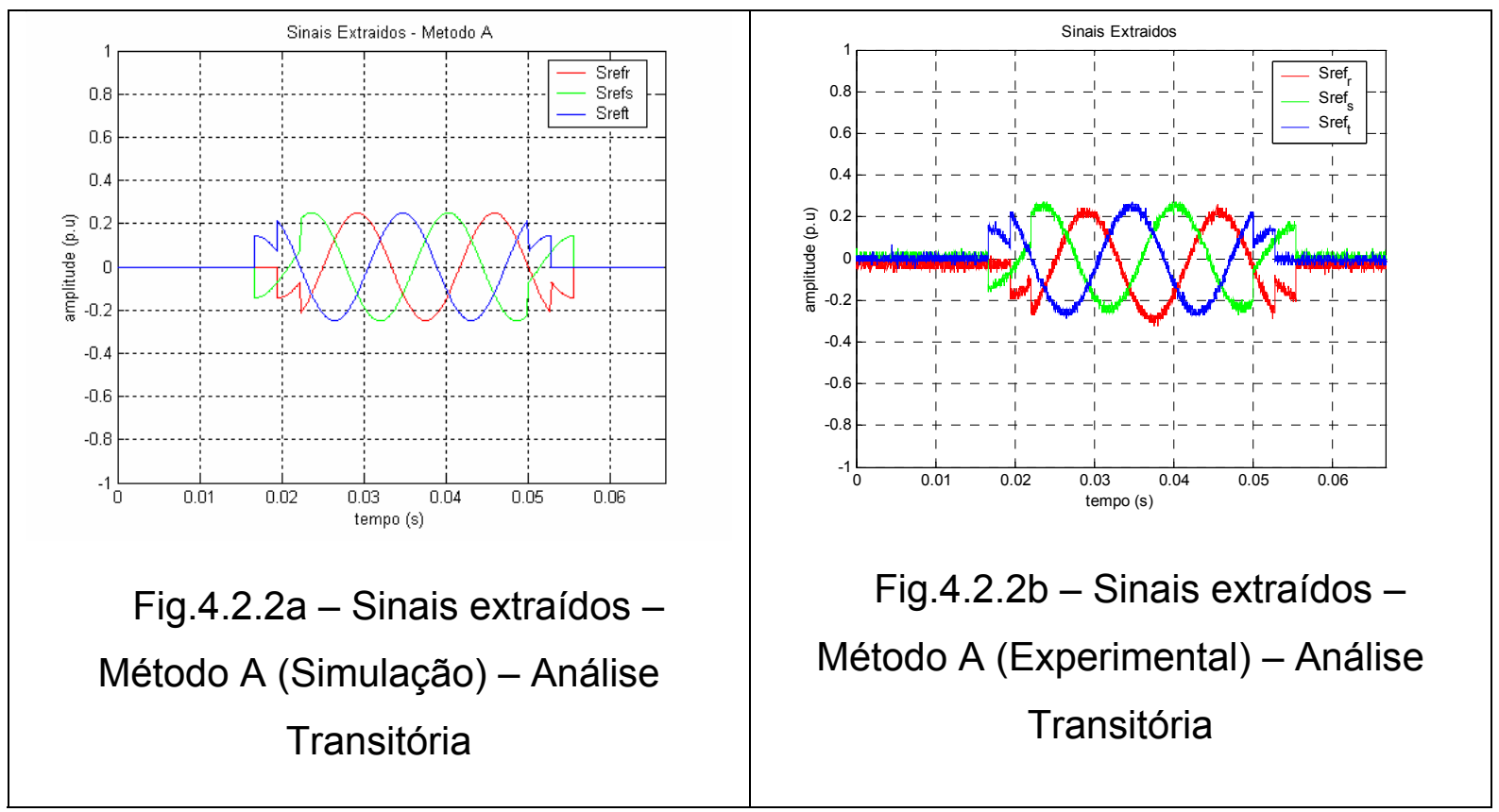




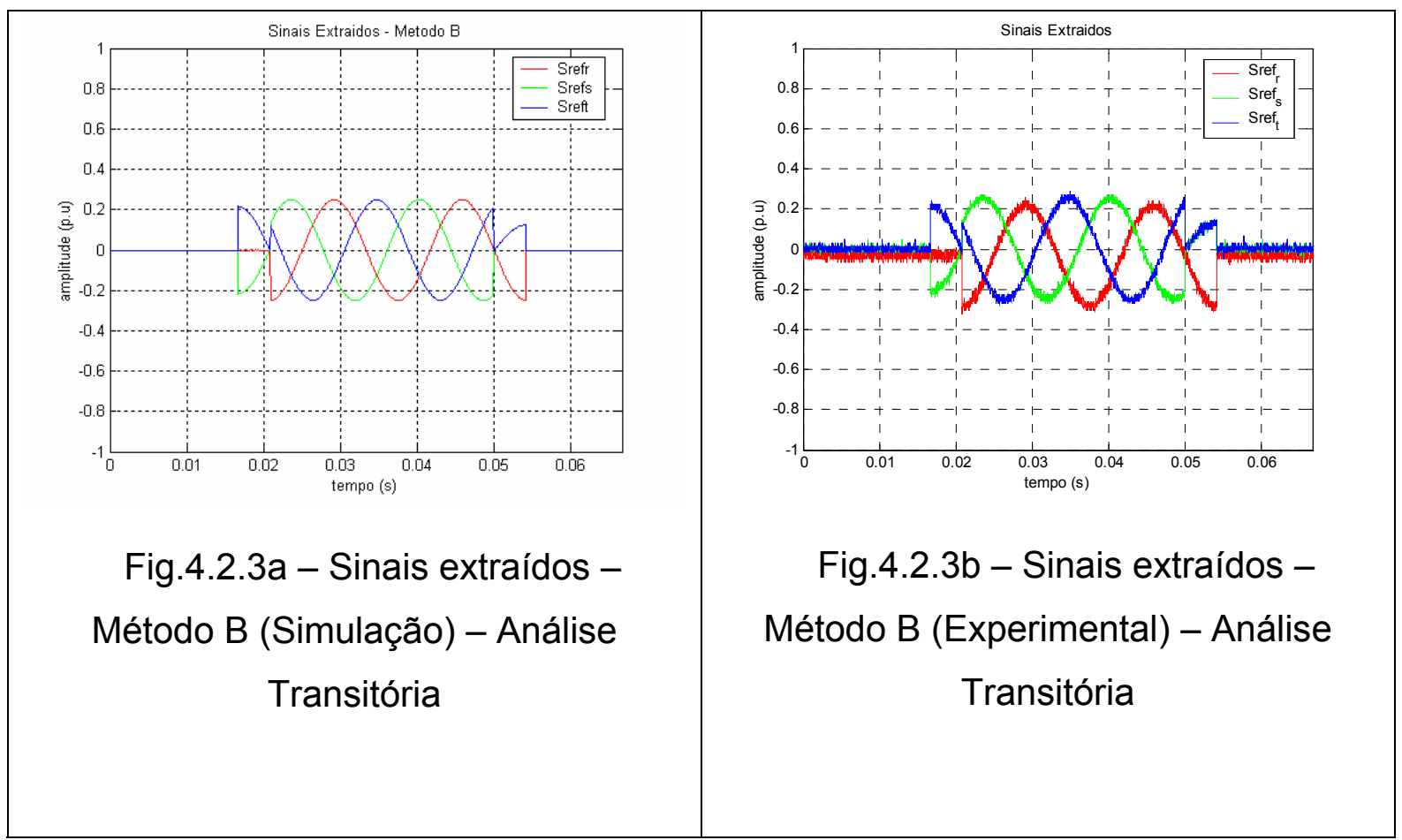

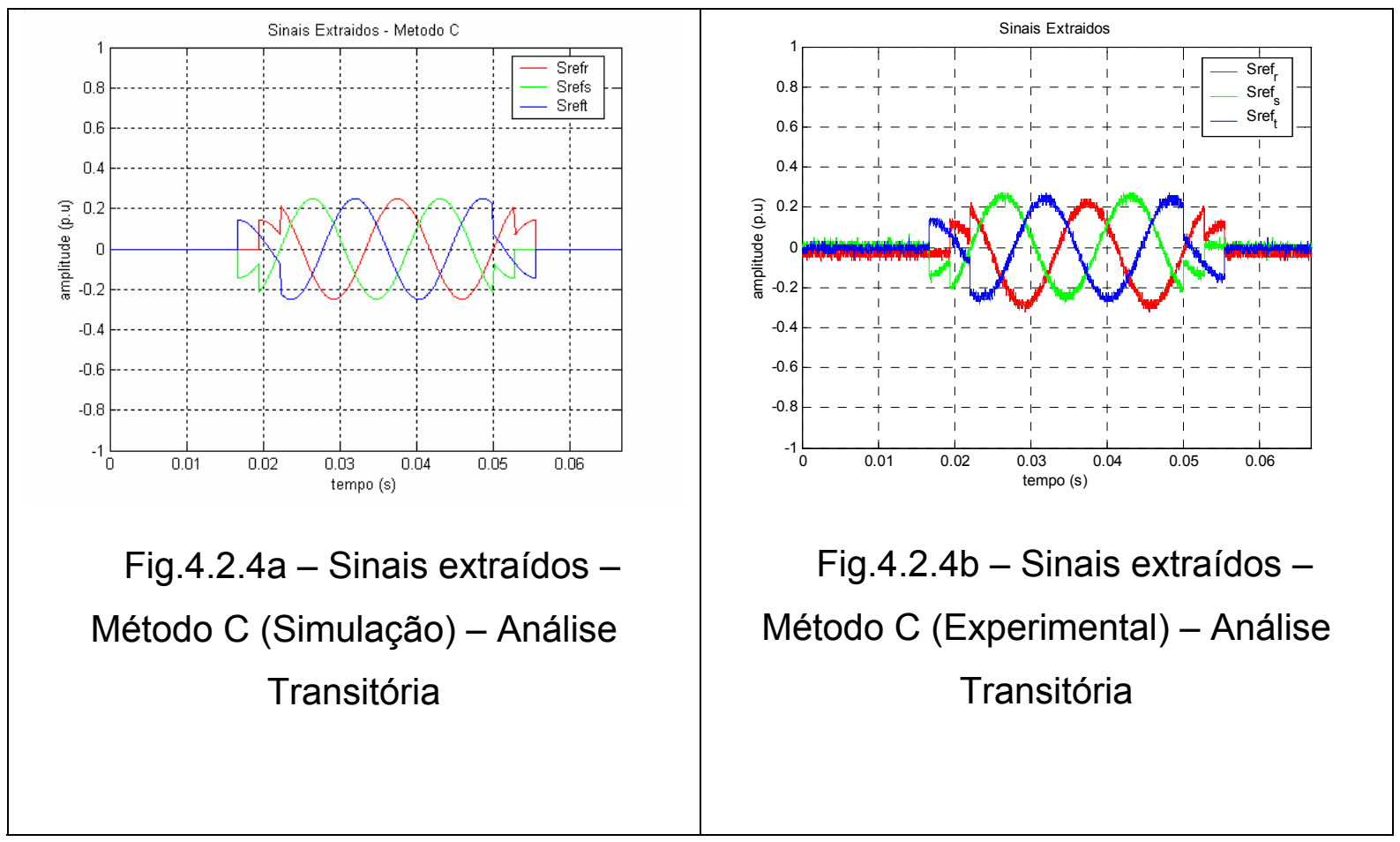




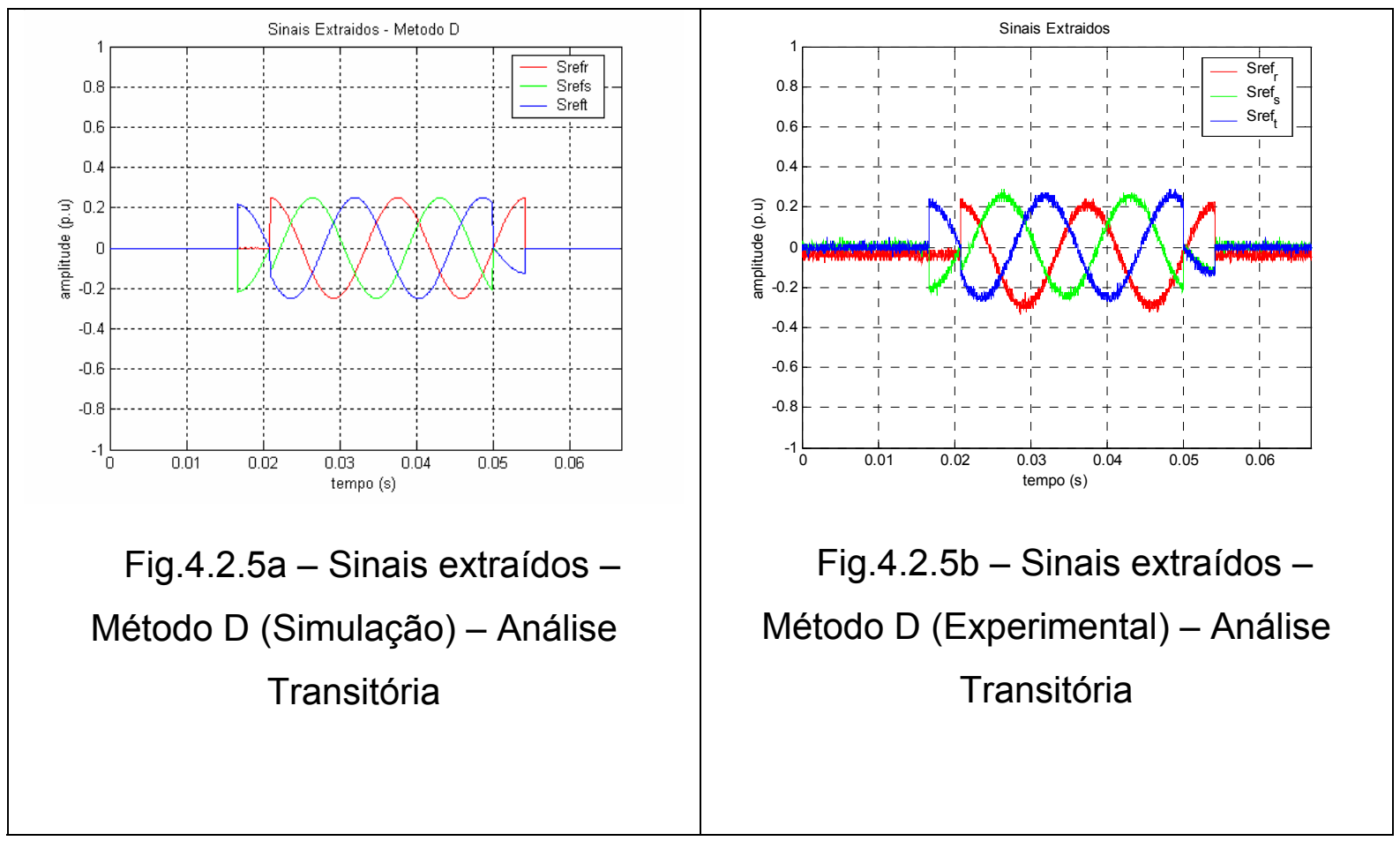

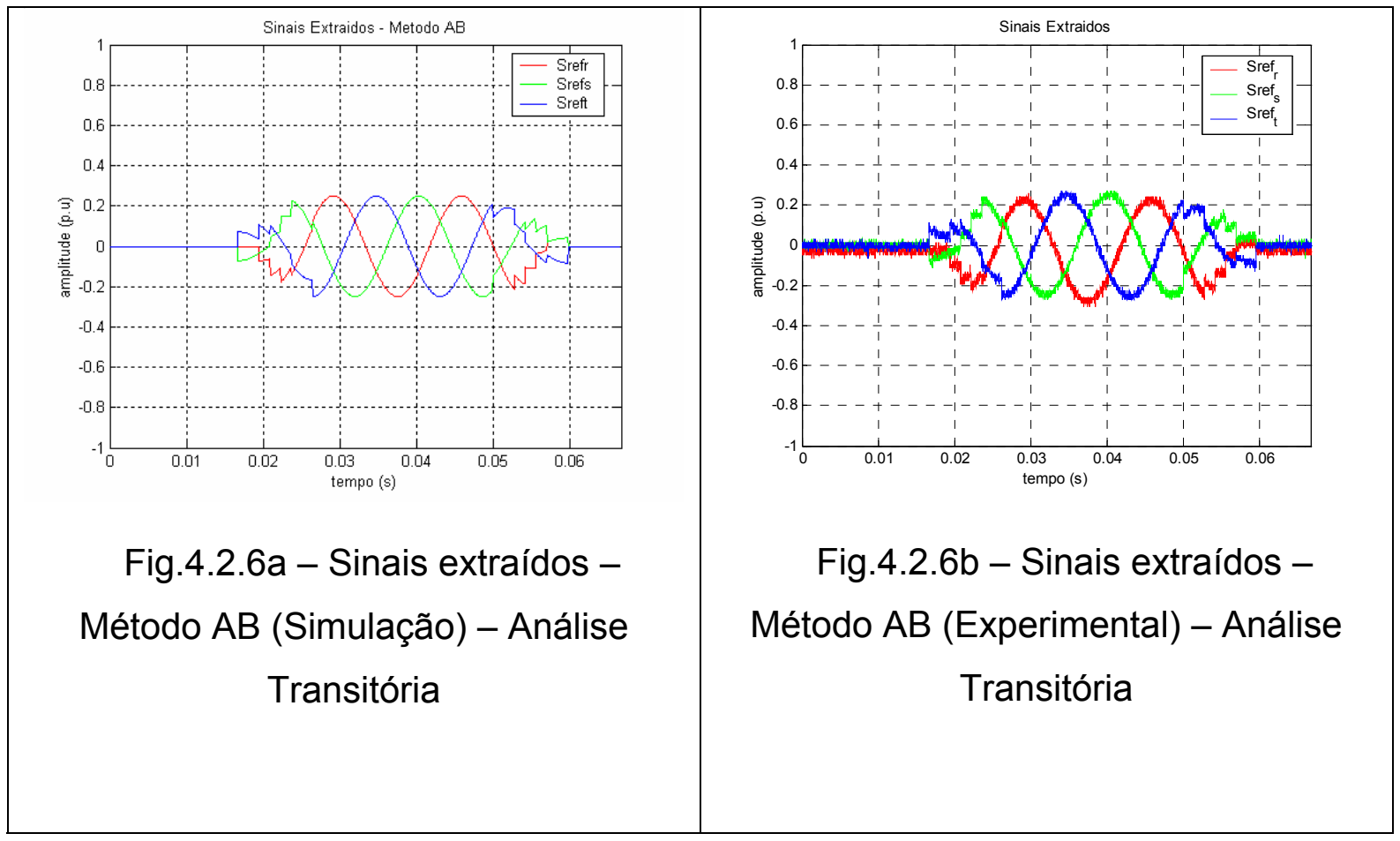




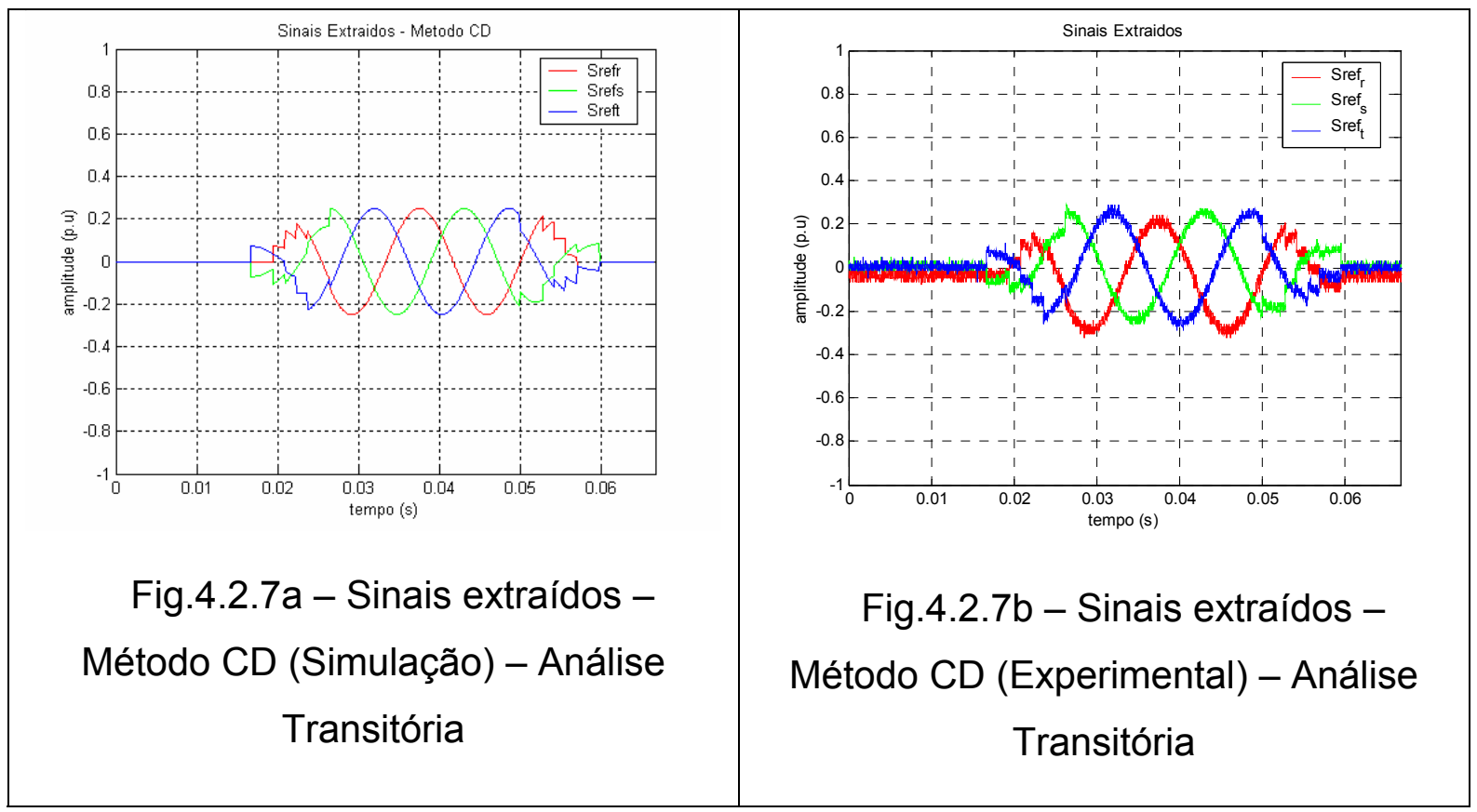

Observa-se pelas Figs.4.2.2, a 4.2.7 que o transitório esperado via simulação foi obtido experimentalmente validando assim os métodos propostos.

Os vários degraus observados (Figs.4.2.2 à 4.2.7) durante os transitórios são conseqüência da forma de implementação dos diversos métodos. O intervalo de tempo para a ocorrência de cada degrau varia de acordo com a operação realizada, de forma condizente com o atraso imposto em cada operação. Os sinais são inicialmente armazenados em um buffer (Apêndice B) e somente então são utilizados para o cálculo das operações.

Na Tabela VII a seguir, apresenta-se, a partir dos tempos medidos das Figs. 4.2.2 à 4.2.7, a duração do transitório de cada método em relação a um ciclo da fundamental tendo como base a freqüência de $60 \mathrm{~Hz}$.

Tabela VII- Duração do Transitório em relação a um ciclo da fundamental (base $60 \mathrm{~Hz}$ ) [para regime permanente]

\begin{tabular}{|l|r|r|r|r|r|r|}
\hline \multicolumn{1}{|c|}{ Método } & $\mathrm{A}$ & $\mathrm{B}$ & $\mathrm{C}$ & $\mathrm{D}$ & $\mathrm{AB}$ & $\mathrm{CD}$ \\
\hline Teórico & $1 / 3$ & $1 / 4$ & $1 / 3$ & $1 / 4$ & $3 / 5$ & $3 / 5$ \\
\hline Simulado & $1 / 3$ & $1 / 4$ & $1 / 3$ & $1 / 4$ & $3 / 5$ & $3 / 5$ \\
\hline Experimental & $1 / 3$ & $1 / 4$ & $1 / 3$ & $1 / 4$ & $3 / 5$ & $3 / 5$ \\
\hline
\end{tabular}


Pode-se observar pela tabela que o tempo obtido experimentalmente correspondeu ao previsto teoricamente em função do tempo necessário para armazenamento das amostras em cada método:

Operações A e C : utilizam um atraso de $90^{\circ}$ demandando um tempo de armazenamento de $1 / 3$ de ciclo

Operações B e D : utilizam um atraso de $120^{\circ}$ demandando um tempo de armazenamento de $1 / 4$ de ciclo

Operações AB e CD : utilizam um atraso de $90^{\circ}$ em cascata com outro atraso de no $120^{\circ}$ demandando um tempo de armazenamento de $3 / 5$ de ciclo

Observa-se também que os métodos apresentam uma rápida estabilização (menor que um ciclo), o que os tornam atrativos para diversas aplicações onde a extração de seqüências positiva e negativa sejam necessárias.

\subsection{Influências da variação da freqüência da rede}

As operações propostas (A, B, C, D) assumem que o período do sinal processado é conhecido, de modo a permitir a obtenção dos sinais em atraso ou avanço ( $\left(\mathrm{s}_{\mathrm{r}-}\right.$ $\left.{ }_{90}(\mathrm{t}), \mathrm{S}_{\mathrm{s}-90}(\mathrm{t}), \mathrm{S}_{\mathrm{t}-90}(\mathrm{t})\right),\left(\mathrm{S}_{\mathrm{r}-60}(\mathrm{t}), \mathrm{S}_{\mathrm{s}-60}(\mathrm{t}), \mathrm{S}_{\mathrm{t}-60}(\mathrm{t})\right),\left(\mathrm{S}_{\mathrm{r} 60}(\mathrm{t}), \mathrm{S}_{\mathrm{s} 60}(\mathrm{t}), \mathrm{S}_{\mathrm{t} 60}(\mathrm{t})\right)$. Deste modo, tendo em vista que as operações demandam a amostragem dos sinais para sua realização, a manutenção do $n^{\circ}$ de amostras adequado a cada atraso pode ser obtida por meio de um circuito de sincronismo (PLL) que impõe uma variação da freqüência de amostragem de acordo com a variação da freqüência da rede ou por meio da expansão ou retração da janela de amostragem conforme a variação da freqüência da rede e conseqüentemente a utilização de valores interpolados ou extrapolados a partir dos valores obtidos.

Para verificar a possibilidade de operação sem sincronismo apresenta-se a seguir a simulação da resposta em freqüência dos operadores e suas combinações (A, $\mathbf{B}$, C, D, AB, CD). 
A influência da variação da freqüência da rede na resposta dos sinais extraídos é analisada verificando-se o comportamento das operações A, B, C, D, AB, CD (baseadas no período fixo de $1 /(60 \mathrm{~Hz})$ ) para o cálculo da componente de seqüência positiva e negativa com um sinal de freqüência compreendida entre $50 \mathrm{~Hz}$ e $70 \mathrm{~Hz}$ (passo de $1 \mathrm{~Hz}$ ) (Figs. 4.3.1 a 4.3.6 - índice (a) relativo ao sinal de teste que contém harmônicos impares e (b) para o que contém harmônicos pares) .

Para a operação $\mathbf{A}$ espera-se, segundo a tabela I (capítulo 3), que $1^{\circ}-, 5^{\circ}+, 7^{0}-$ tenham ganho unitário e as demais tenham ganho nulo. Observa-se (Fig. 4.3.1) que para um sinal de $60 \mathrm{~Hz}$, com janela fixa de 1/60s isso ocorre, no entanto, quando a freqüência do sinal se afasta de $60 \mathrm{~Hz}$, a operação $\mathbf{A}$ apresenta ganho menor que 1 para $1^{\circ}-5^{\circ}+7^{\circ}-e$, com defasagem crescente, já os outros harmônicos impares que eram totalmente eliminados, passam a ter ganho não nulo, aparecendo na saída da operação A. O mesmo ocorre para os demais casos. De modo geral, observa-se nas Figs. 4.3.1 a 4.3.6 que, dependendo do método utilizado, a amplitude na extração da seqüência positiva ou negativa da fundamental apresenta erros para uma grande faixa de variação $(50$ a $70 \mathrm{~Hz})$ do sinal de entrada. A defasagem e a variação na atenuação dos harmônicos, dependendo do método, também são significativas, o que remete à necessidade de se verificar a viabilidade de uso do método em determinadas aplicações.

Observando-se a Resolução ANEEL 791/02 (ONS 2002), que estabelece que em condições normais de operação, em regime permanente, a freqüência do sistema interligado deve se situar entre $59,9 \mathrm{~Hz}$ e $60,1 \mathrm{~Hz}$, discuti-se a influência da variação da freqüência da rede, nesta faixa de operação, na resposta dos sinais extraídos verificando-se o comportamento da operação $A B$ visto que para as outras operações a análise será semelhante (Fig.. 4.3.7).

Pode-se observar (Fig.. 4.3.7) que a amplitude não foi afetada pela variação da freqüência mantendo-se estável ao longo de toda a faixa de variação, no entanto, o mesmo não ocorre com a fase que apresenta uma variação de aproximadamente +$8^{\circ}$ para uma variação de $+-0,83 \%(+-0.5 \mathrm{~Hz})$ na freqüência fundamental. Analisandose a Fig. 4.3.7 (padrão de variação da ONS) e a Fig. 4.3 .5 (variação de $50-70 \mathrm{~Hz}$ ) observa-se que a influência da freqüência é não linear e que afeta tanto a amplitude quanto a fase obtida pela operação realizada. 
Assim, um PLL pode ser utilizado para seguir instantaneamente o período do sinal analisado. Se um PLL não for utilizado, e for utilizada uma janela com período fixo, poderão aparecer erros de amplitude e fase. A operação sem PLL é somente factível para sinais com pequena variação de freqüência, o que ocorre em equipamentos conectados a redes elétricas fortes, característica de sistemas interligados. Para sistemas, por exemplo, alimentados por gerador Diesel, pode ser necessária a operação com PLL, para a determinação do período do sinal em tempo real. $O$ circuito de sincronismo PLL pode ser implementado de diversas maneiras (Santanu et al., 2003), (Marafão F.P. et al., 2004),(Matakas Jr.,L et al., 2006) sempre buscando-se um compromisso entre a estabilidade e a velocidade do sincronismo.
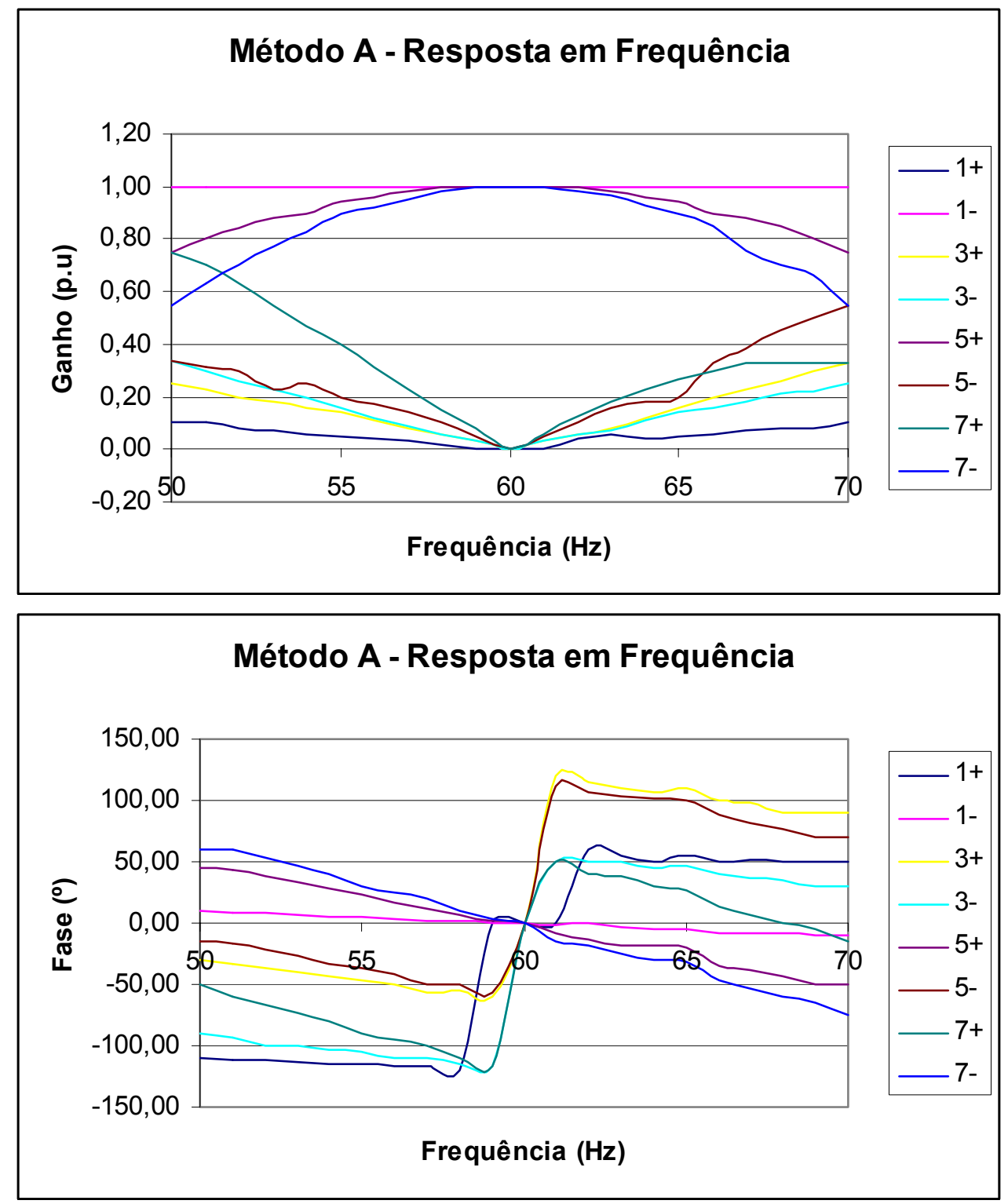

Fig. 4.3.1a - Operação A - Resposta em Freqüência para vários harmônicos (impares) e seqüências. Acima: ganho Abaixo:fase 

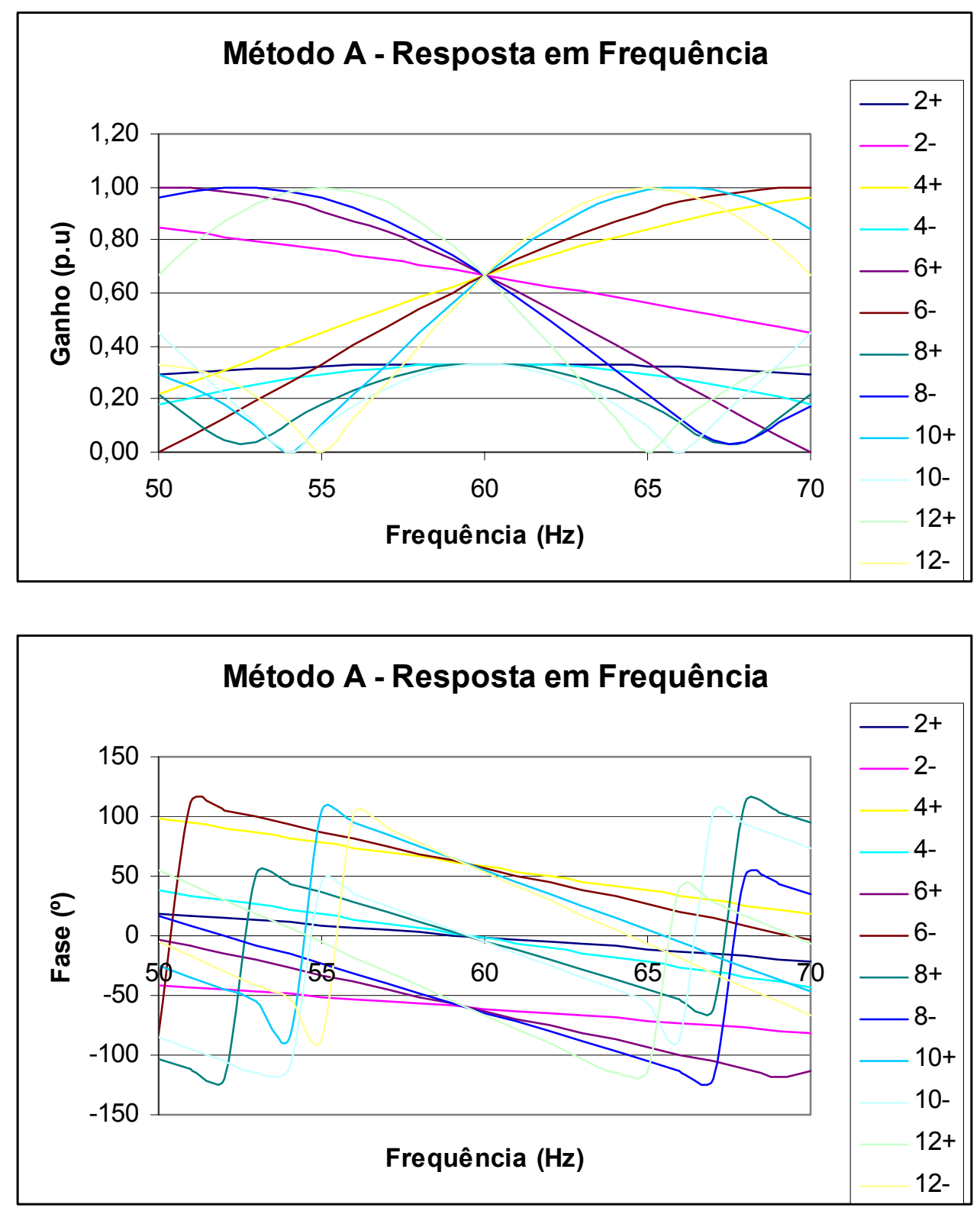

Fig. 4.3.1b - Operação A - Resposta em Freqüência para vários harmônicos (pares) e seqüências. Acima: ganho Abaixo:fase 

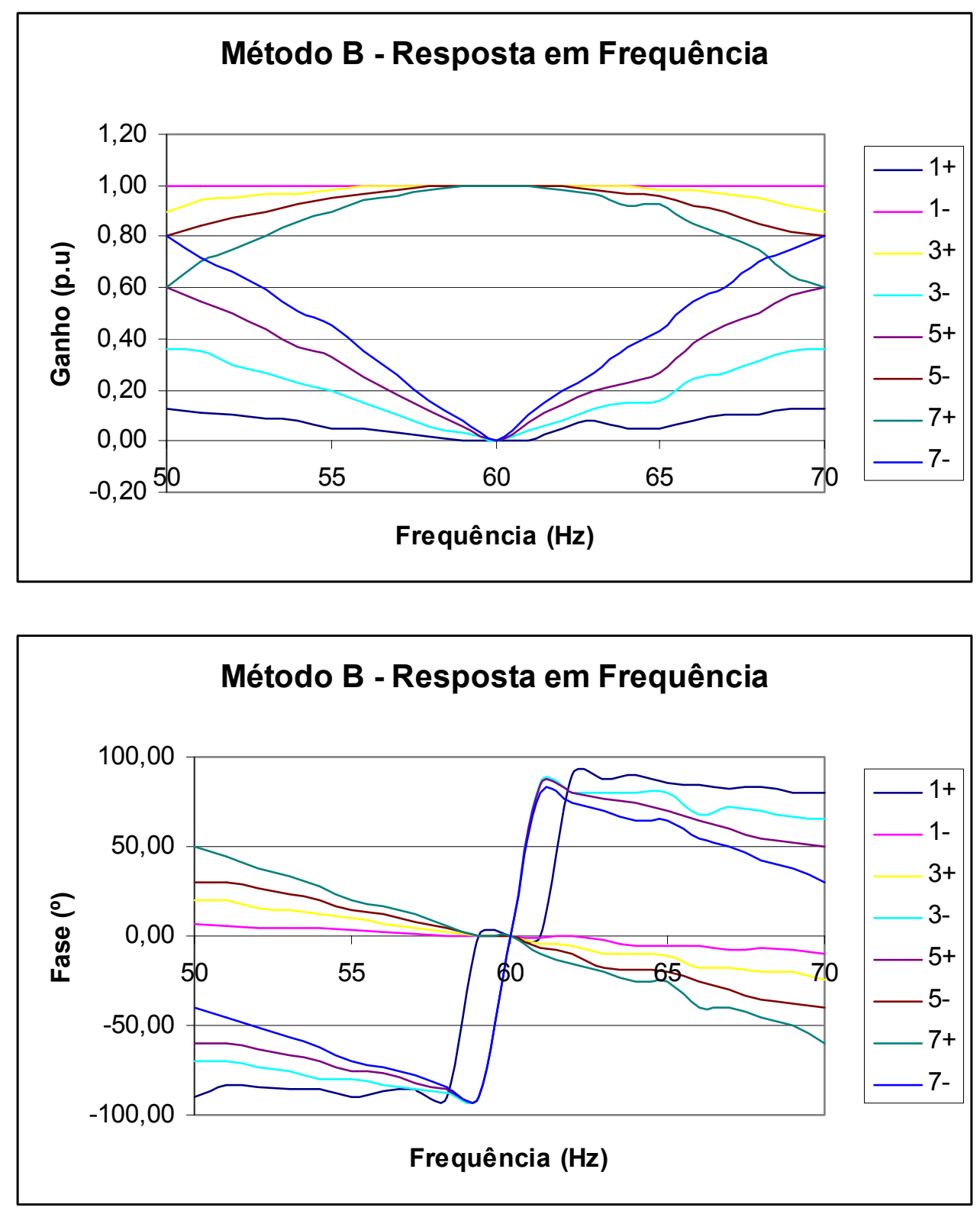

Fig. 4.3.2a - Operação B - Resposta em Freqüência para vários harmônicos (impares) e seqüências. Acima: ganho Abaixo:fase 

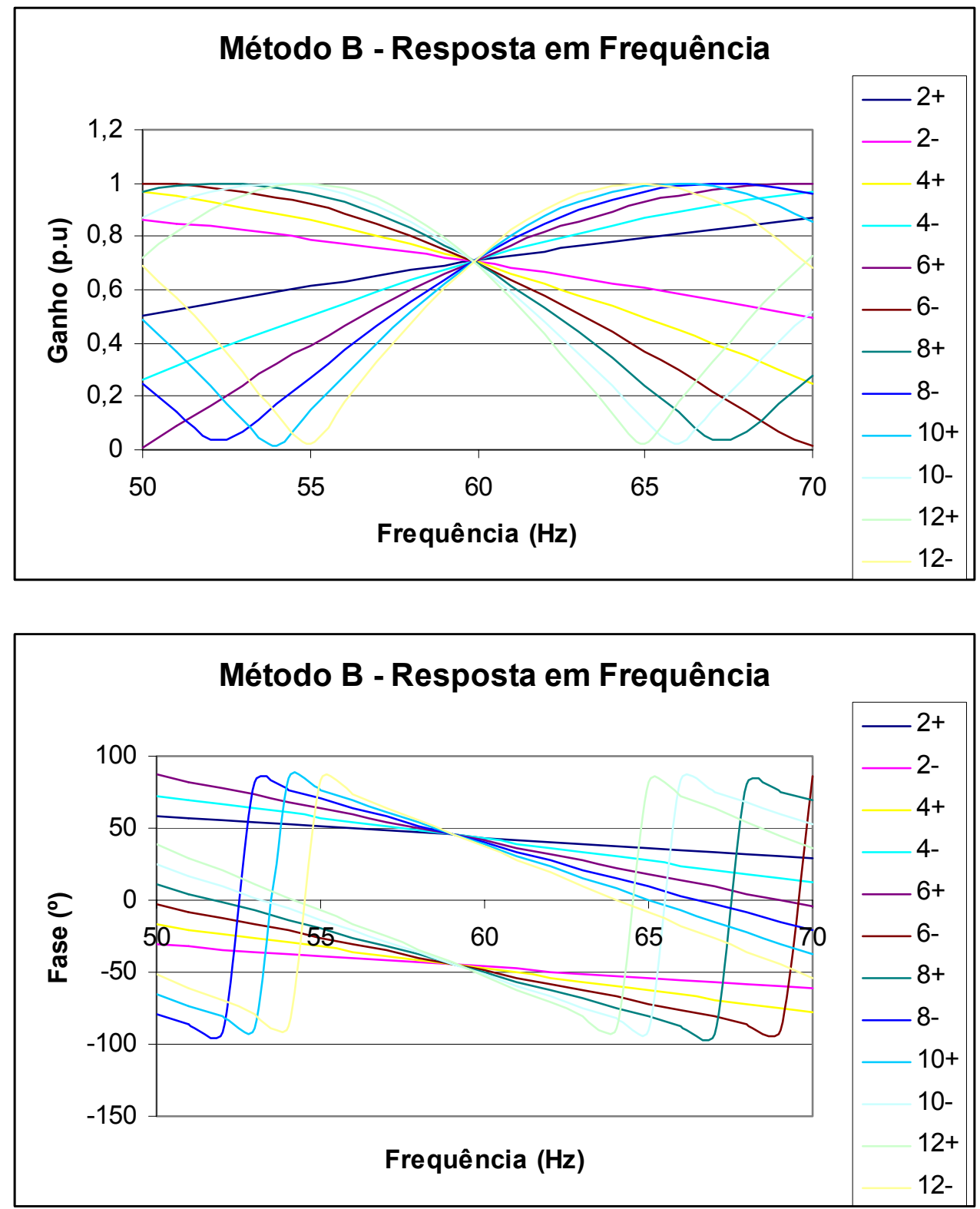

Fig. 4.3.2b - Operação $B$ - Resposta em Freqüência para vários harmônicos (pares) e seqüências. Acima: ganho Abaixo:fase 

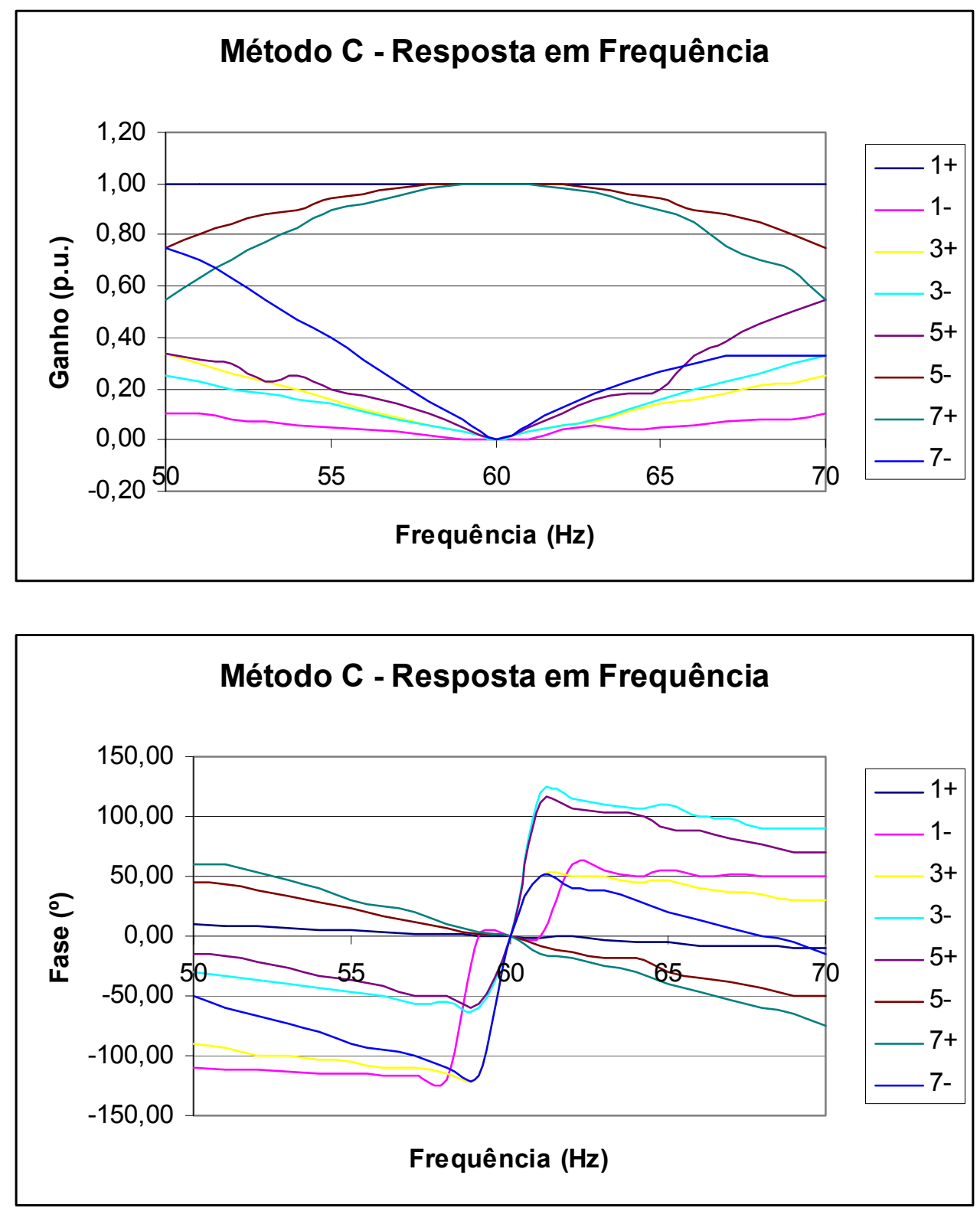

Fig. 4.3.3a - Operação $C$ - Resposta em Freqüência para vários harmônicos (impares) e seqüências. Acima: ganho Abaixo:fase 

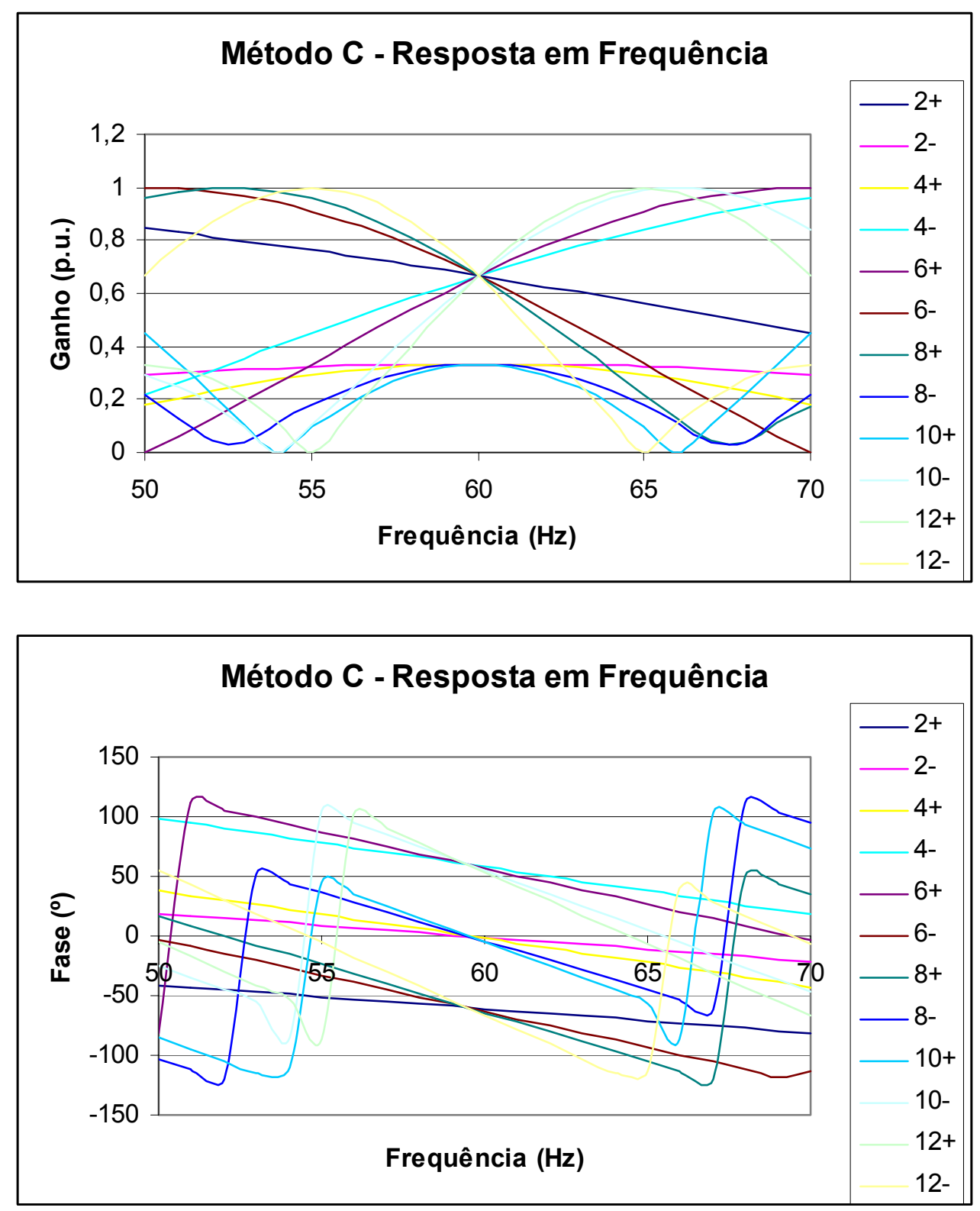

Fig. 4.3.3b - Operação $C$ - Resposta em Freqüência para vários harmônicos (pares) e seqüências. Acima: ganho Abaixo:fase 

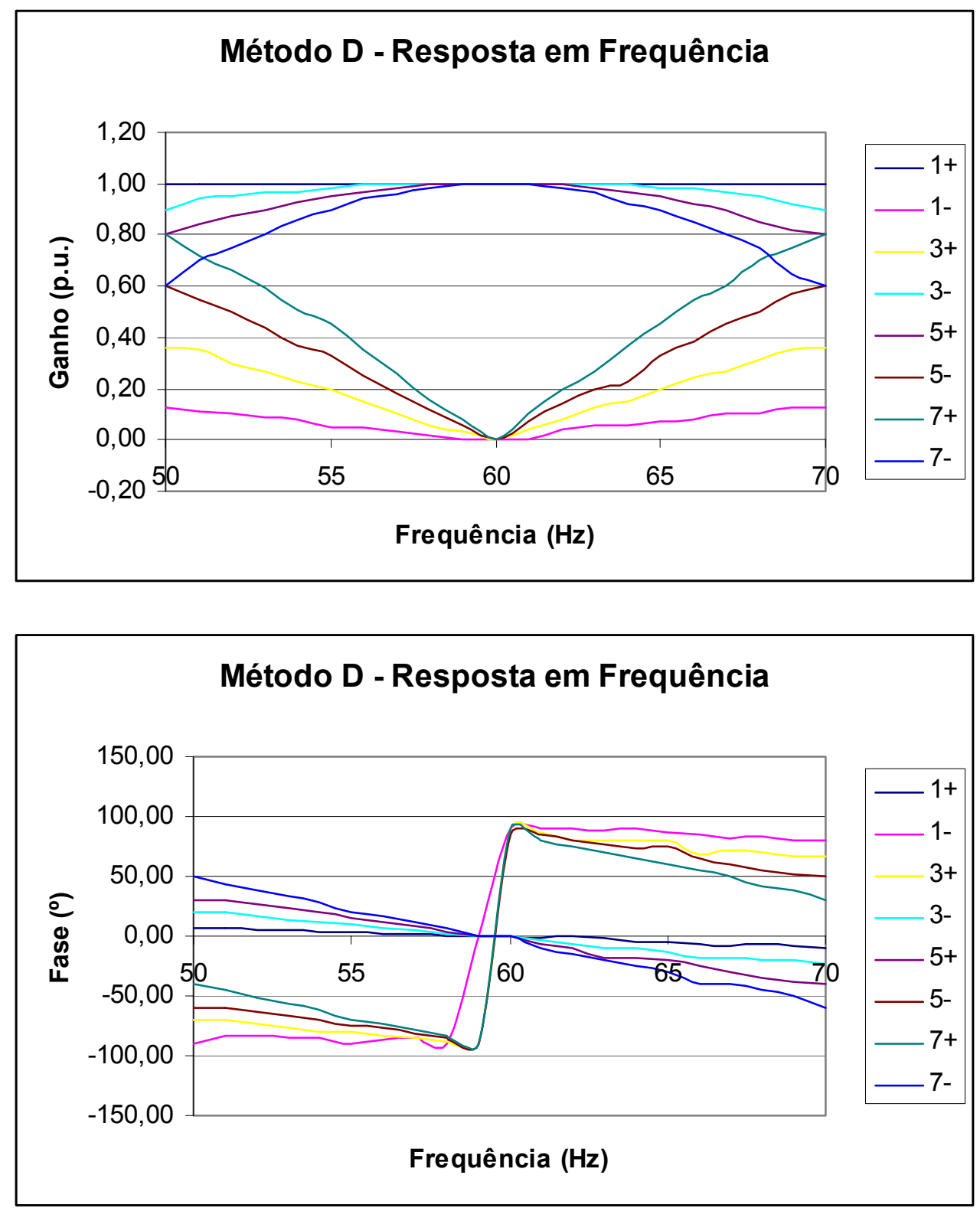

Fig. 4.3.4a - Operação D - Resposta em Freqüência para vários harmônicos (impares) e seqüências. Acima: ganho Abaixo:fase 

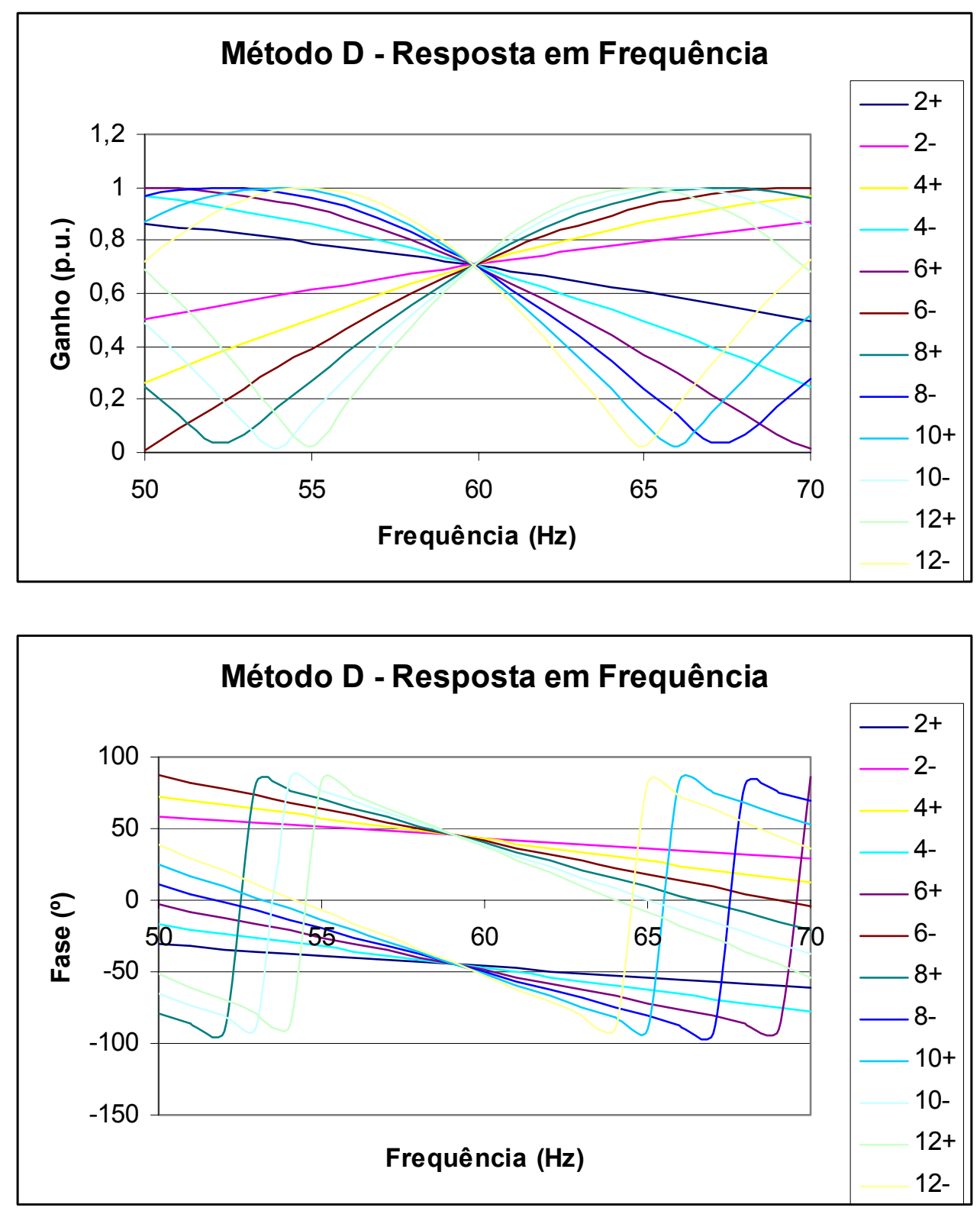

Fig. 4.3.4b - Operação D - Resposta em Freqüência para vários harmônicos (pares) e seqüências. Acima: ganho Abaixo:fase 

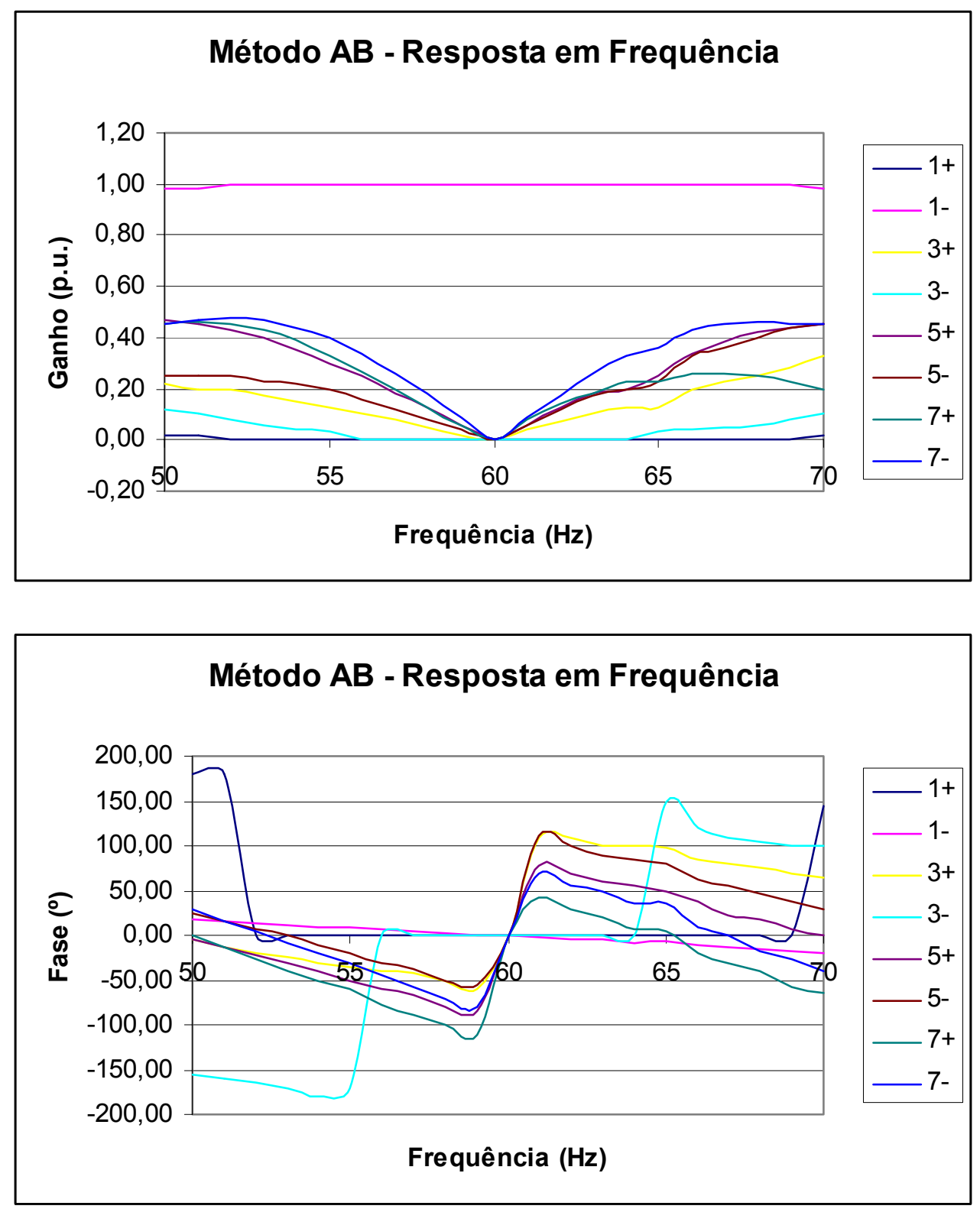

Fig. 4.3.5a - Operação $A B$ - Resposta em Freqüência para vários harmônicos (impares) e seqüências. Acima: ganho Abaixo:fase 

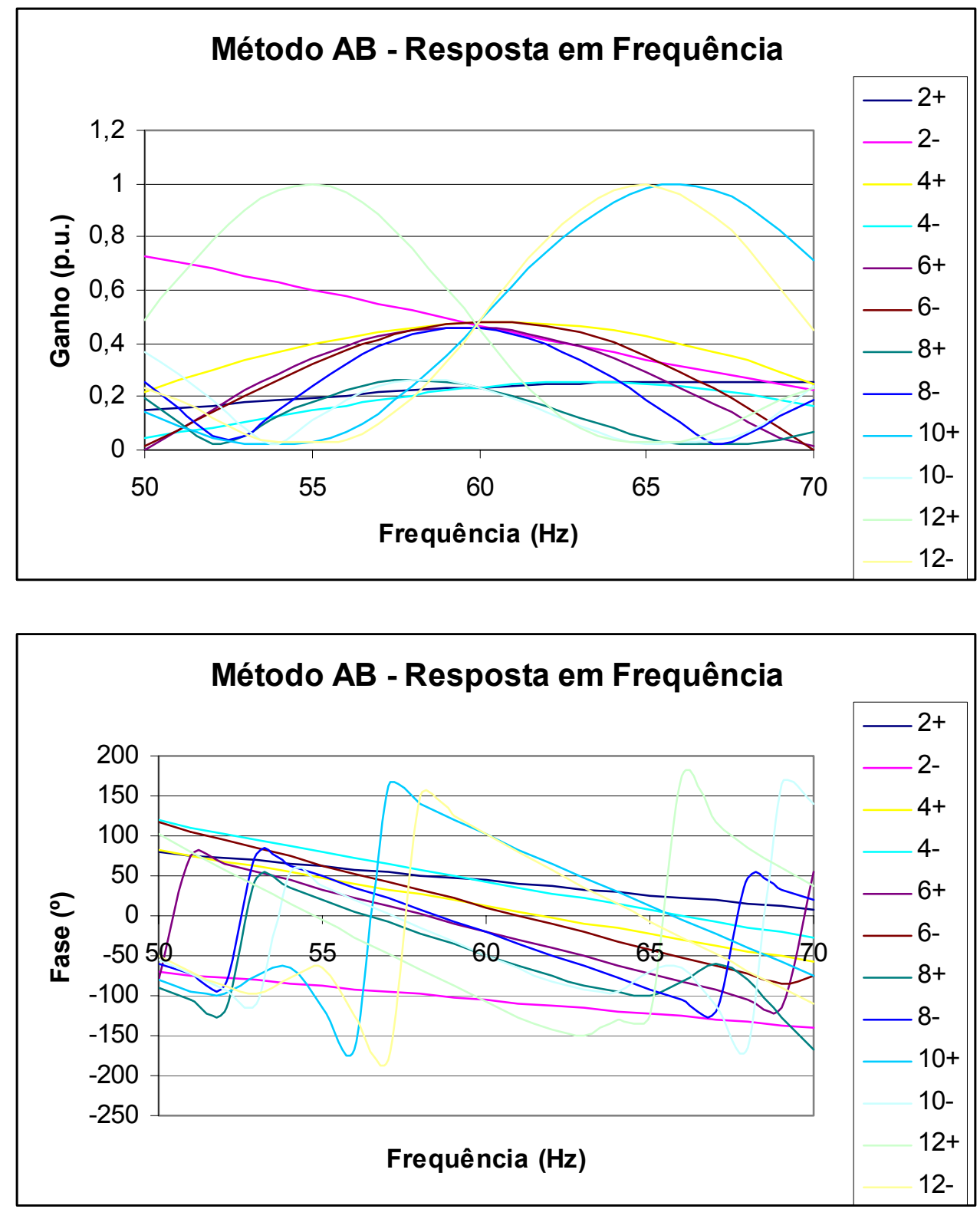

Fig. 4.3.5b - Operação $A B$ - Resposta em Freqüência para vários harmônicos (pares) e seqüências. Acima: ganho Abaixo:fase 

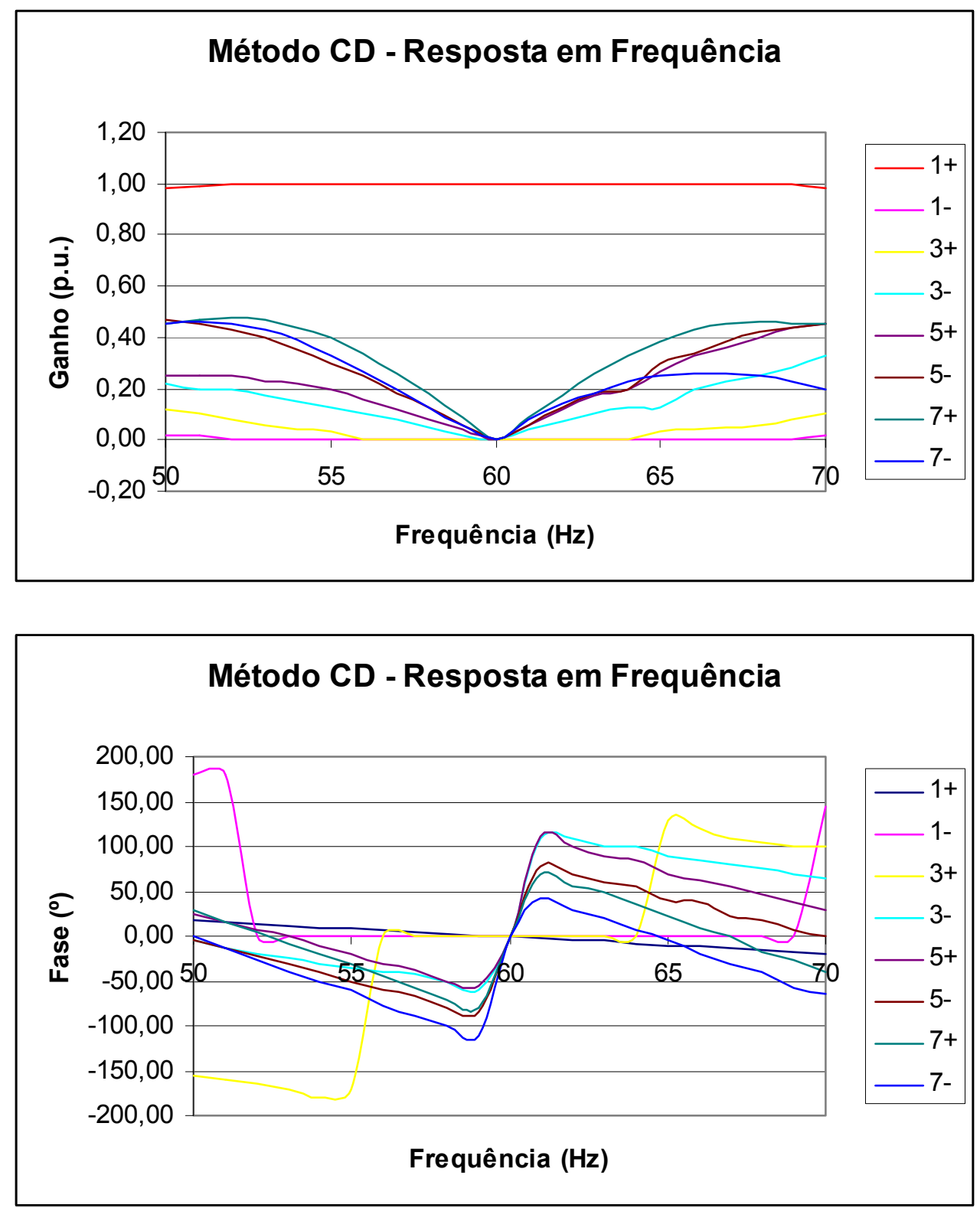

Fig. 4.3.6a - Operação CD - Resposta em Freqüência para vários harmônicos (impares) e seqüências. Acima: ganho Abaixo:fase 

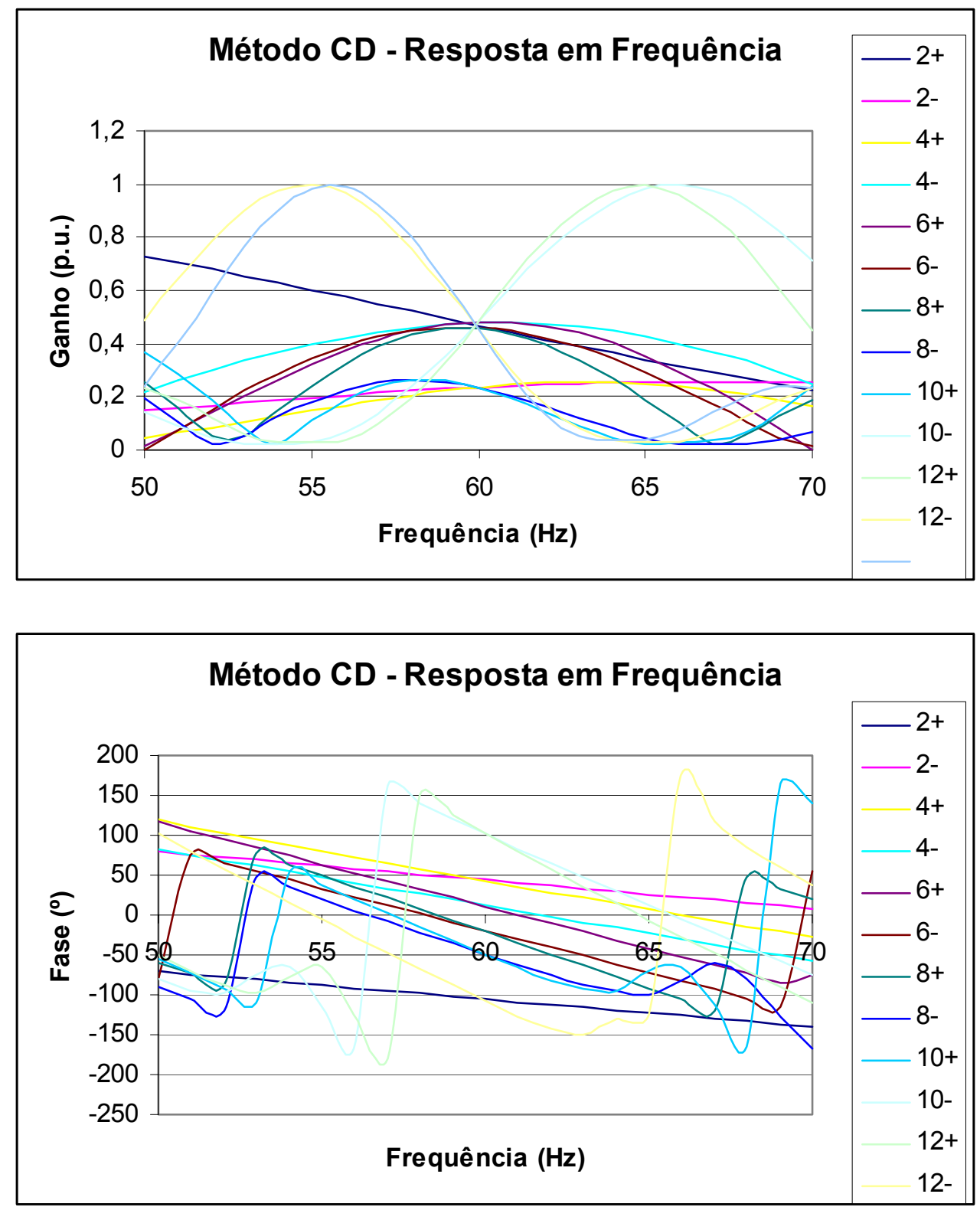

Fig. 4.3.6b - Operação CD - Resposta em Freqüência para vários harmônicos (impares) e seqüências. Acima: ganho Abaixo:fase 

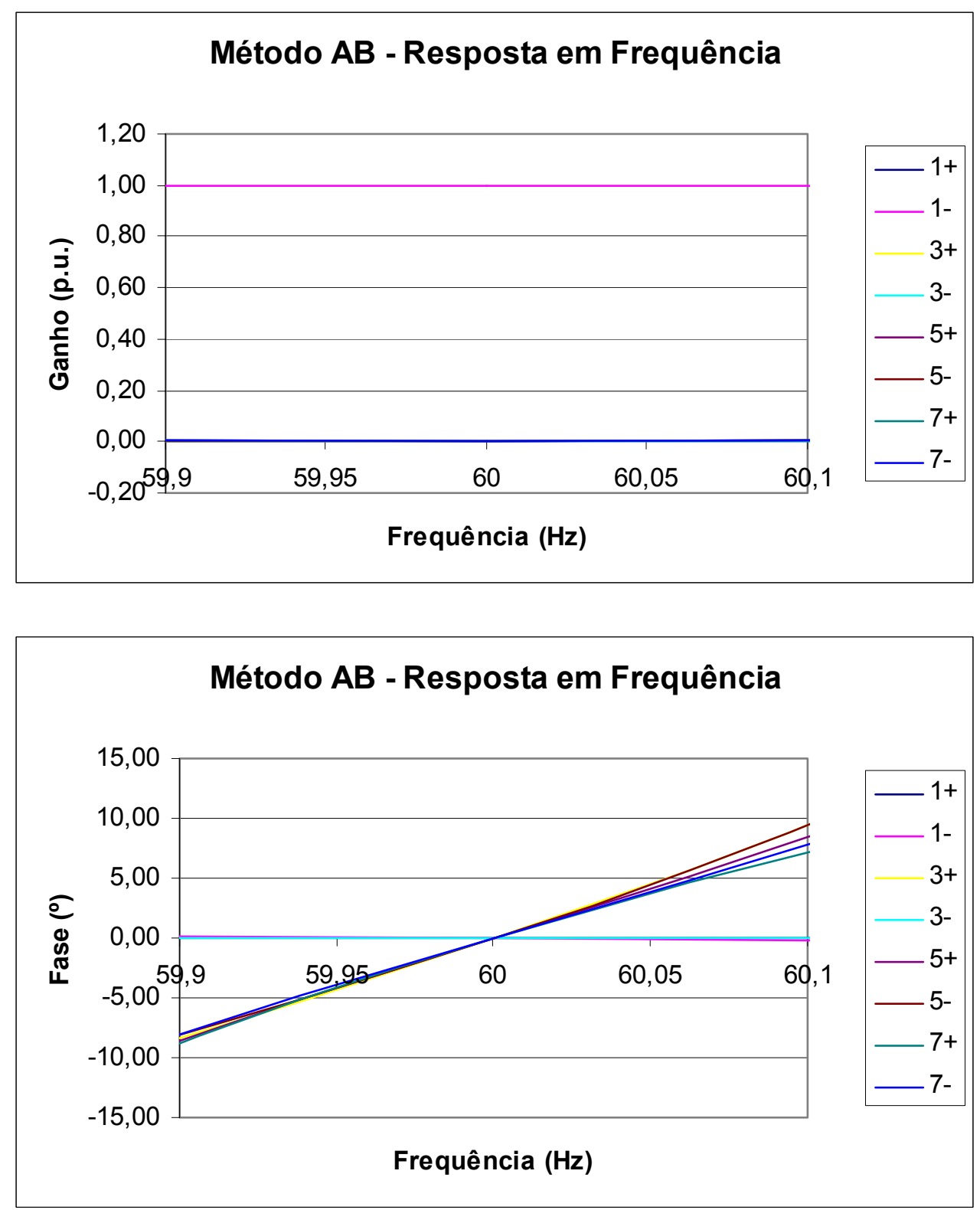

Fig. 4.3.7a - Operação $A B$ - Resposta em Freqüência (PADRÃO ONS) para vários harmônicos (impares) e seqüências. Acima: ganho Abaixo:fase 

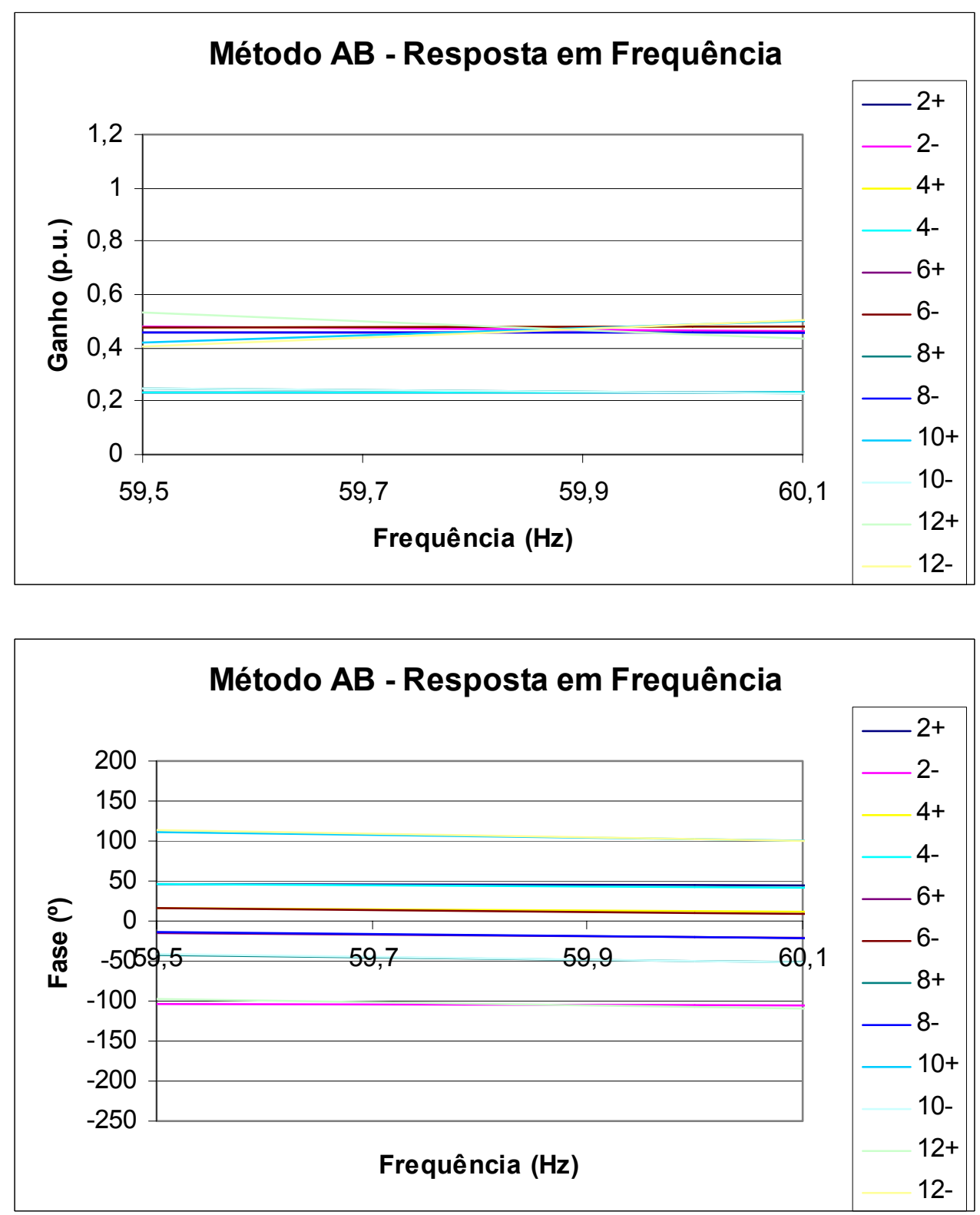

Fig. 4.3.7b - Operação AB - Resposta em Freqüência (PADRÃO ONS) para vários harmônicos (pares) e seqüências. Acima: ganho Abaixo:fase 


\subsection{Tempos de execução}

Para medir o tempo de execução do programa no DSP, monitorou-se uma variável interna que recebe o valor zero no início do programa e, no final, recebe o valor um. Os resultados obtidos são apresentados na Tabela VIII.

Tabela VIII - Tempo de execução de cada método implementado no DSP ADM401

\begin{tabular}{|c|c|c|c|c|c|c|}
\hline Método & A & B & C & D & AB & CD \\
\hline $\begin{array}{c}\text { Tempo de execução do programa no } \\
\text { DSP (us) }\end{array}$ & 7.6 & 9 & 7.6 & 9 & 15.4 & 15.4 \\
\hline
\end{tabular}

\subsection{Observações gerais}

A partir das análises efetuadas, observa-se que os métodos propostos:

- $\quad$ funcionam como um filtro digital com tempo de atuação bem definido;

- $\quad$ podem ou não necessitar de bloco de sincronismo;

- $\quad$ operam diretamente com os sinais medidos;

- $\quad$ apresentam um tempo de estabilização relativamente rápido;

- apresentam uma baixa complexidade numérica resultando em um tempo de execução pequeno;

- $\quad$ permitem uma extração efetiva das seqüências requeridas na freqüência fundamental, além de adicionalmente poderem atuar como detectores de harmônicos.

- $\quad$ extraem parcialmente os harmônicos pares e apresentam variação de ganho e fase quando da variação da freqüência 


\section{APLICAÇÕES DO EXTRATOR}

Os resultados da simulação de dois exemplos de aplicação do extrator proposto são apresentados.

Aplicações relacionadas à compensação ativa de perturbações (filtros ativos de potência, restauradores dinâmicos de tensão) e relés de proteção necessitam da detecção em tempo real de desequilíbrios e harmônicos, de tensão ou de corrente (Abellán et al., 1999), (Bhavaraju; Enjeti, 1993), (Akagi; Nabae, 1993), (Brittain, 2002), (Chen; Hsu, 2000), (Choi et al., 2000), (Cutri; Matakas Jr., 2003), (Czarnecki, 1993), (Dixon et al., 1995), (Hochgraf; Lasseter, 1998), (Kim et al., 2001), (Lin et al., 1992), (Kim, 2002), (Chang,G.W.; Shee,T., 2002), (Ledwich; George, 1994), (Matsui; Fukao, 1990), (McGranagham,M., 2003), (Nikolaenko, 1998), (Senini; Wolfs, 2000), (Verdelho; Marques, 1994), (Watanabe; Aredes, 1998), (Willems, 1993), (Zhang; Xu, 2001), (Cutri; Matakas Jr., 2004) e (Ahn,S.U. et al., 2005).

Neste capítulo são exemplificadas as aplicações dos algoritmos propostos (cap.3) através da simulação de um filtro ativo de potência (seção 5.1) e simulação de um relé de seqüência negativa (seção 5.2).

Os circuitos utilizados na simulação são apresentados no apêndice $C$ e estão disponíveis no $C D$ que acompanha a tese.

\subsection{EXEMPLO DE APLICAÇÃO DO EXTRATOR - SIMULAÇÃO DE UM FILTRO ATIVO DE POTÊNCIA}

A compensação ativa de desequilíbrios pode ser feita através da injeção de correntes de seqüência negativa através de conversores autocomutados do tipo PWM (Lin et al., 1992), (Bhavaraju; Enjeti, 1993), (Verdelho; Marques, 1994), (Abellán et al., 1994), (Dixon et al., 1995), (Hochgraf; Lasseter, 1998), (Watanabe; Aredes, 1998), (C.C. Chen; Hsu, 2000), (Choi et al., 2000), (Senini; Wolfs, 2000), (Zhang; Xu, 2001), (Kim, 2002) e (Cutri, 2004). O diagrama em blocos do sistema de compensação ativa utilizando um conversor comutado do tipo PWM é apresentado na Fig.5.1-1. 


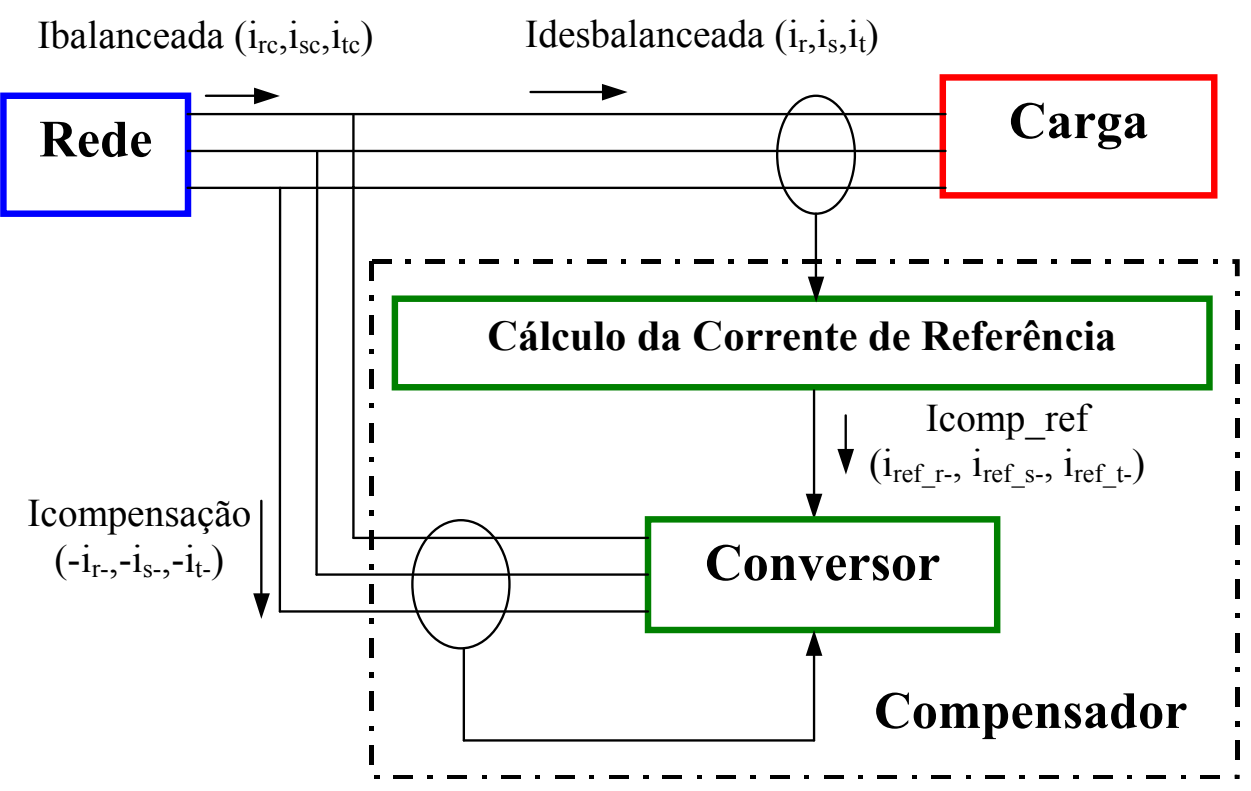

Fig. 5.1-1 - Diagrama em blocos do compensador ativo de desequilíbrios

As correntes da carga $\left(i_{r}(t), i_{s}(t), i_{t}(t)\right)$ são medidas e um algoritmo efetua o cálculo das correntes de referência ( $\left.i_{\text {ref_r- }}, i_{\text {ref_s--, }} i_{\text {ref_t- }}\right)$ a serem injetadas $\left(i_{r-}(t), i_{s-}(t), i_{t-}(t)\right)$ pelo conversor, de modo que as correntes resultantes na rede $\left(\mathrm{i}_{\mathrm{rc}}(\mathrm{t}), \mathrm{i}_{\mathrm{sc}}(\mathrm{t}), \mathrm{i}_{\mathrm{tc}}(\mathrm{t})\right)$ sejam balanceadas. As correntes injetadas $\left(i_{r_{-}}(t), i_{s-}(t), i_{t-}(t)\right)$ são controladas a fim de se garantir que sigam com a maior fidelidade possível os valores de referência calculados (i iref_r- $_{\text {r- }} \mathrm{i}_{\text {ref_s-s, }} \mathrm{i}_{\text {ref }} \mathrm{t}-$-).

Neste exemplo de aplicação optou-se pela utilização de um conversor do tipo VSI nas simulações do compensador completo. O processo de equacionamento para o dimensionamento do conversor e das malhas de tensão e corrente é o mesmo utilizado em (Cutri, 2004).

A seguir, apresenta-se na Fig.5.1-2 um diagrama em blocos mostrando o conversor e as malhas de controle de tensão no barramento CC e da corrente CA. A Tabela IX apresenta um quadro-resumo com as opções adotadas para a simulação completa de um sistema de compensação de desequilíbrios. 


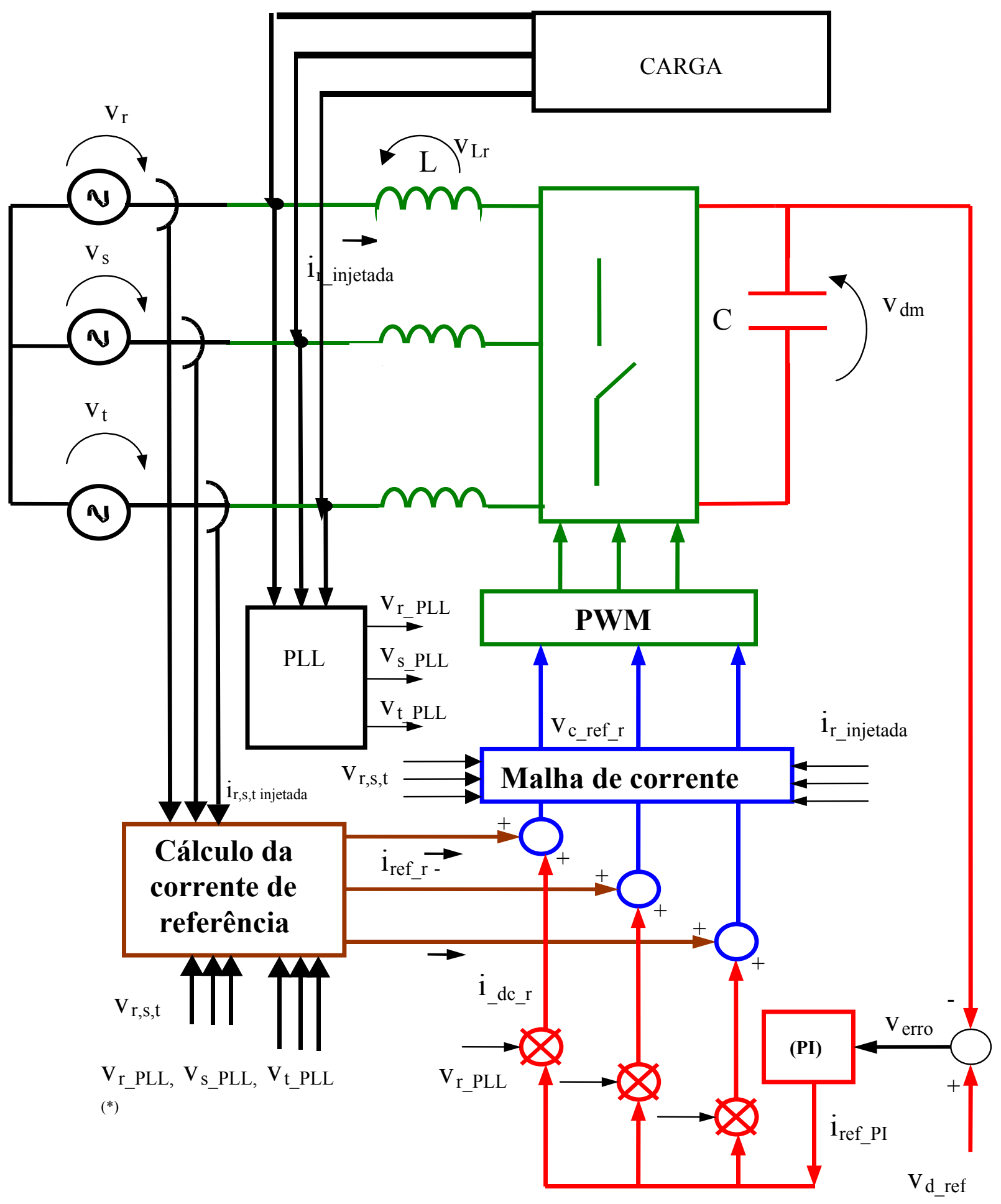

Fig.5.1-2 - Diagrama em bloco mostrando o conversor e as malhas de controle de tensão no barramento $\mathrm{CC}$ e da corrente $\mathrm{CA}$ vermelho-malha de tensão $\mathrm{CC}$ / azul-malha de corrente $\mathrm{CA}$ / verde-conversor / marrom-cálculo da corrente de referência 
Tabela IX - Quadro resumo das características adotadas referentes ao compensador para a simulação completa do sistema

\begin{tabular}{|c|c|}
\hline Conversor & VSI \\
\hline Malha de corrente & Deadbeat \\
\hline Modulação & $\begin{array}{l}\text { PWM com portadora triangular } \\
\text { assimétrico } \mathrm{f}_{\mathrm{C}}=\mathrm{f}_{\mathrm{a}} / 2\end{array}$ \\
\hline $\begin{array}{l}\text { Método para cálculo da corrente de } \\
\text { referência }\end{array}$ & $\begin{array}{l}\text { A (Fig.5.1-3) } \\
\text { CD (Fig.5.1-4) }\end{array}$ \\
\hline Tensão de pico de fase $\left[\mathrm{V}_{\mathrm{p}}\right](\mathrm{V})$ & 1 \\
\hline Corrente de seqüência negativa [I-] (A) & 1 \\
\hline Freqüência da rede [f] $(\mathrm{Hz})$ & 60 \\
\hline $\begin{array}{l}\text { Impedância equivalente da rede [Z] (por } \\
\text { fase) }\end{array}$ & $10 \%$ \\
\hline Indutância de Acoplamento [L] (uH) & 265 \\
\hline $\begin{array}{l}\text { Resistência em série com a indutância de } \\
\text { acoplamento }(\Omega)\end{array}$ & $10 \%$ de $X_{L}$ \\
\hline Freqüência de chaveamento $\left[\mathrm{f}_{\mathrm{c}}\right](\mathrm{Hz})$ & $10 \mathrm{k}$ \\
\hline Frequência de amostragem [ $\left.\mathrm{f}_{\mathrm{a}}\right](\mathrm{Hz})$ & $20 \mathrm{k}$ \\
\hline $\mathrm{K}_{\mathrm{p}}$ & 1.12 \\
\hline $\mathrm{K}_{\mathrm{I}}$ & 27 \\
\hline
\end{tabular}

Para a simulação completa do sistema foi utilizado o software Matlab com passo de integração de $1 \mu$ s.

O sistema de compensação é simulado para uma carga variável ligada em triângulo. Todas as simulações são apresentadas com condições quiescentes em " $\mathrm{t}=0$ " e todas as amplitudes das tensões e correntes são representadas pelos seus valores de pico, exceto quando especificado em contrário.

Apresentam-se a seguir, as formas de onda da corrente na carga, da corrente injetada pelo filtro e na rede após a compensação utilizando o método de extração $\mathbf{A}$ (Fig.5.1-3) e a estratégia de compensação total descrita na fig. 3c (Fig. 5.1-4). 
Os métodos são simulados para uma carga variável ligada em triângulo.

O comportamento da carga simulada pode ser dividido em três partes com as seguintes características:

I. Sistema sem carga é submetido a um degrau de carga que caracteriza o sistema com o máximo desequilíbrio possível (X\%=100\%)

- inicialmente o sistema se encontra sem nenhuma carga até $0.005 \mathrm{~s}$;

- no intervalo de 0,005 s até 0,06 s é ligada uma carga desbalanceada (uma carga monofásica de $1 \Omega$ é ligada entre as fases $R$ e $S$ ), caracterizando uma situação de máximo desequilíbrio possível (a amplitude da seqüência positiva na linha (1 A) é igual a amplitude da seqüência negativa na linha (1 A) );

II. Submete-se o sistema desbalanceado a um degrau de carga de forma a balanceá-lo $(X \%=0 \%)$

- no intervalo de 0,06 s à 0,12 s o sistema recebe um degrau de carga de modo a balanceá-la, tornando-se uma carga em triângulo, com três resistências de $1 \Omega$. Tem-se apenas uma corrente de seqüência positiva, com amplitude de $3 \mathrm{~A}$;

III. Adiciona-se ao sistema uma carga não linear com harmônicos

- após 0,12 s somam-se a cada fase da carga do sistema um sinal harmônico com freqüência de $180 \mathrm{~Hz}$, amplitude igual a 0,5 A e com fases $\left(0^{\circ}\right.$ (fase $r$ ), $120^{\circ}$ (fase $s$ ) e $-120^{\circ}$ (fase $t$ )), em paralelo com sinal harmônico com freqüência de $300 \mathrm{~Hz}$, amplitude igual a 0,2 A e com fases $\left(-30^{\circ}\right.$ (fase $r$ ), $-150^{\circ}$ (fase s) e $90^{\circ}$ (fase $\mathrm{t}$ )), em paralelo com uma resistência $3 \Omega$; 

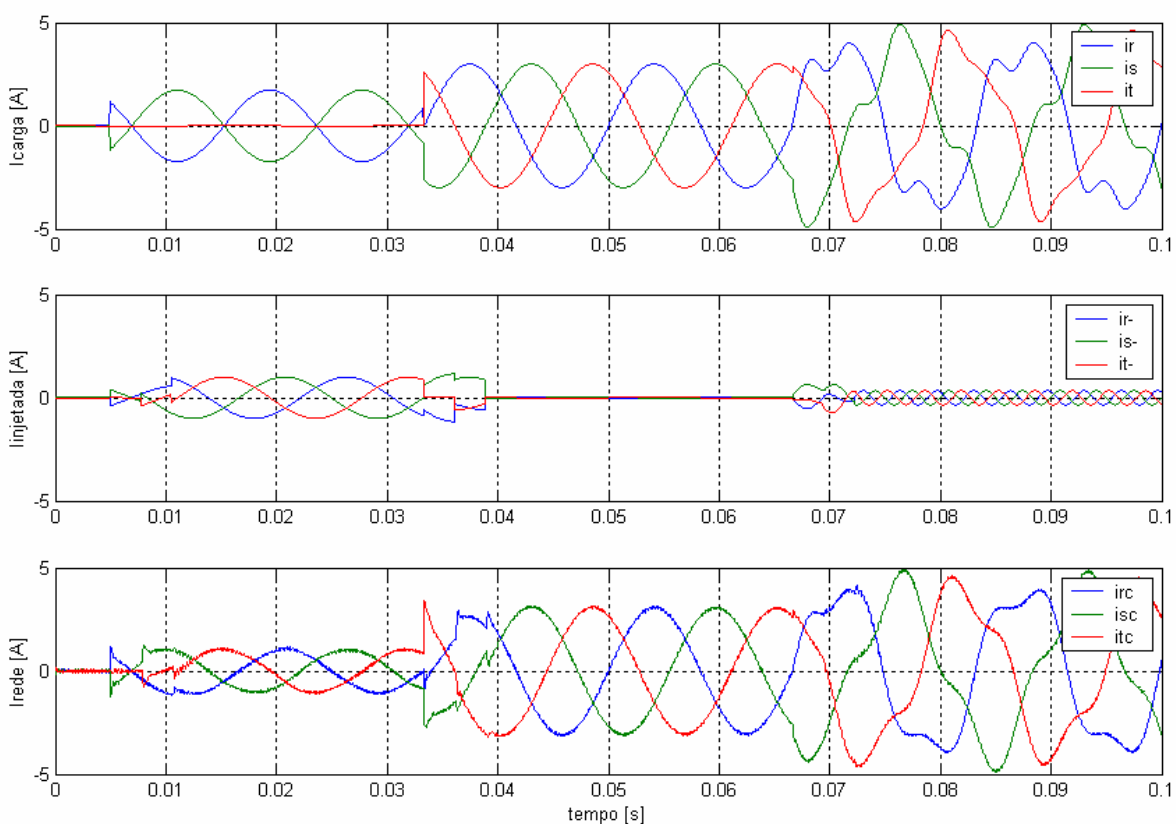

Fig.5.1-3 - Filtro Ativo (Método A)

Correntes da carga (gráfico superior)

Correntes de seqüência negativa extraídas (gráfico intermediário) Correntes de linha na rede após a compensação (gráfico inferior)
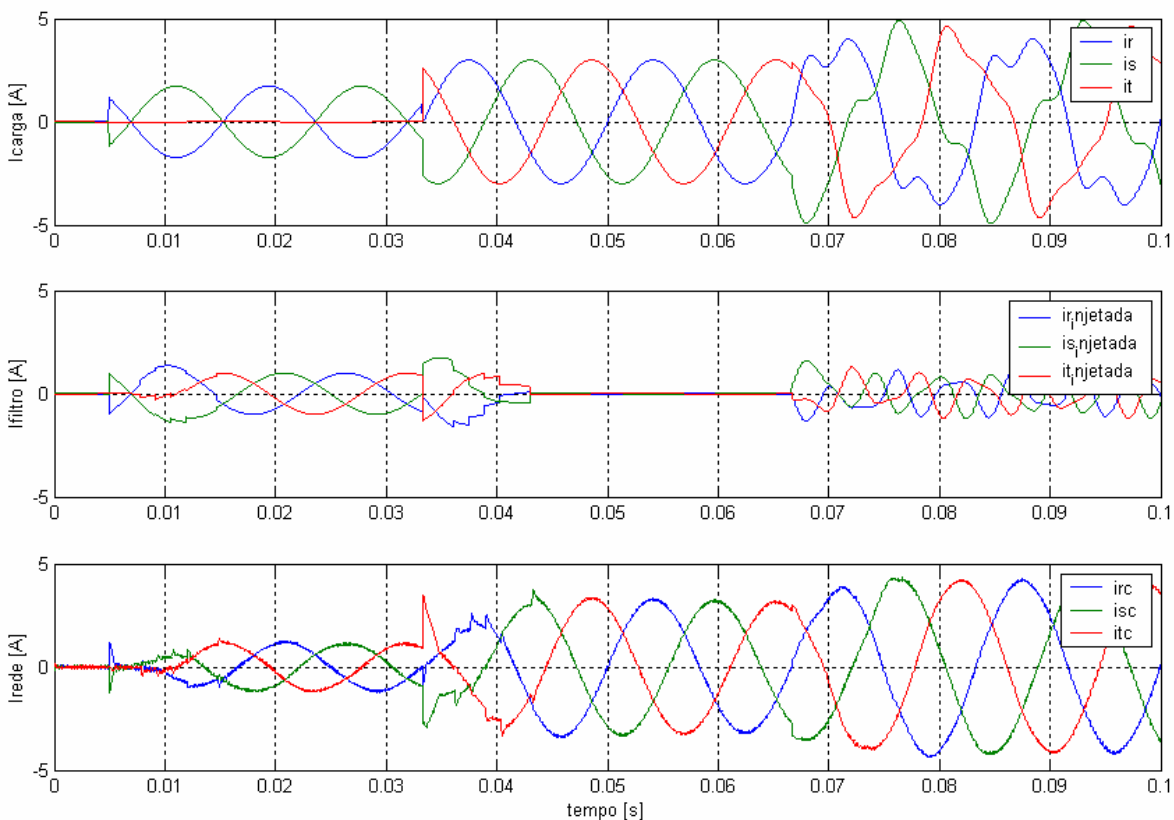

Fig.5.1-4 - Filtro Ativo (Compensação Total) Correntes da carga (gráfico superior)

Correntes de seqüência negativa e harmônicos extraídos (gráfico intermediário) Correntes de linha na rede após a compensação (gráfico inferior) 
Observa-se que:

- pela Fig.5.1-3 que a corrente injetada pelo filtro corresponde ao esperado, sendo injetada a parcela referente apenas à compensação da seqüência negativa da corrente da carga (parte I) bem como a compensação de parte dos harmônicos (parte III). A compensação foi realizada no tempo previsto de $1 / 4$ de ciclo compensando o desequilíbrio do sistema.

- pela Fig.5.1-4 que a corrente injetada pelo filtro corresponde ao esperado, sendo injetada a parcela referente à compensação total (seqüência negativa e harmônicos) da corrente da carga. Observa-se que a compensação foi realizada no tempo previsto de 3/5 de ciclo compensando o desequilíbrio e distorções do sistema.

Na Fig.5.1-5 apresenta-se a atuação da malha de corrente para uma das fases (fase r) (método A). Nota-se o funcionamento esperado da malha de corrente onde a corrente injetada (azul) segue a referência imposta (verde).

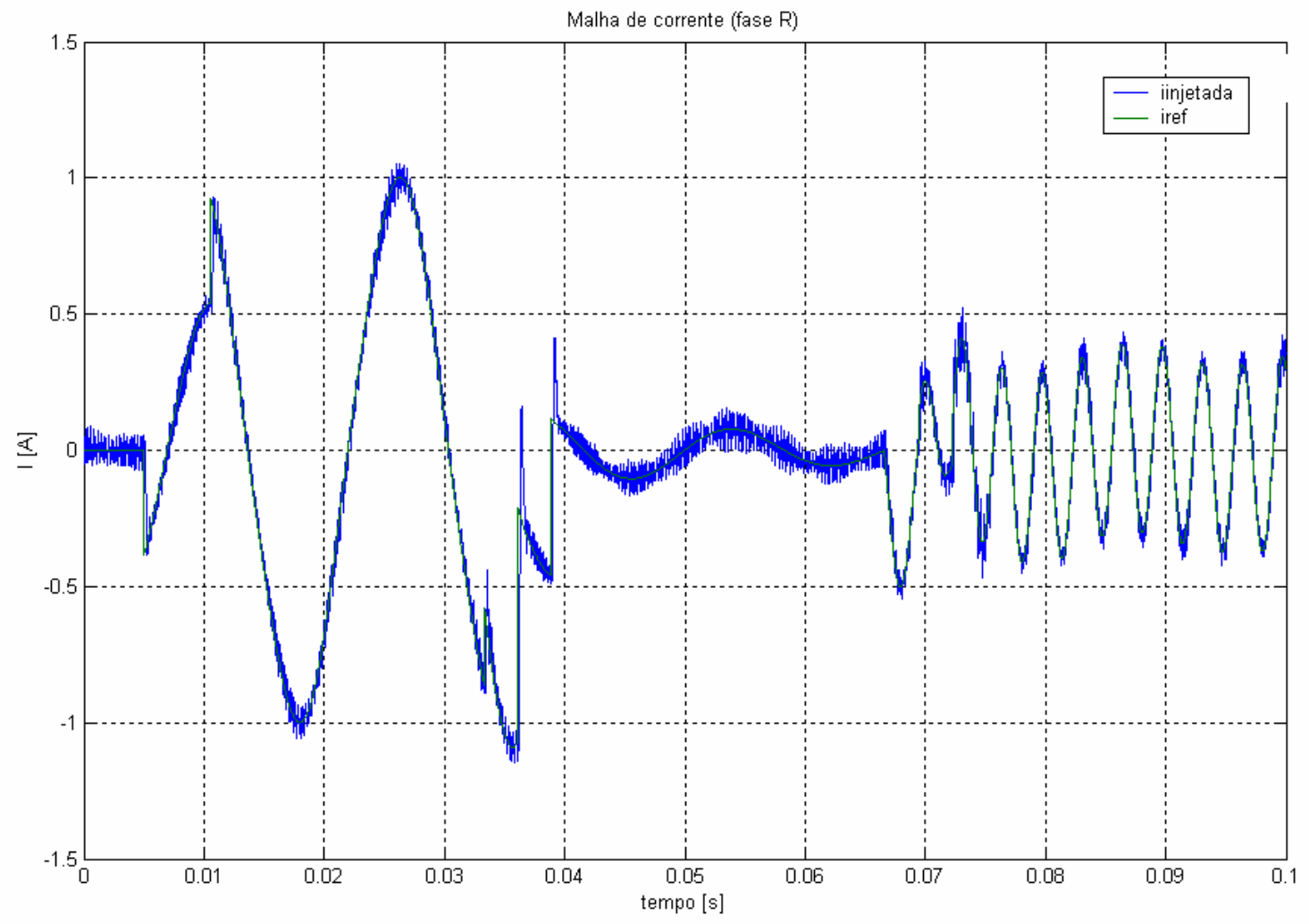

Fig.5.1-5 - Comportamento da malha de corrente (fase r) (Método A) 


\subsection{EXEMPLO DE APLICAÇÃO DO EXTRATOR - SIMULAÇÃO DE UM RELÉ DE SEQUÊNCIA NEGATIVA}

A definição do grau de desequilíbrio de corrente (Robba et al., 1996) em um sistema elétrico, é dada pela eq.(5.2-1).

$$
\mathrm{X}(\%)=\frac{\left|\overrightarrow{\mathrm{I}_{-}}\right|}{\left|\dot{\mathrm{I}}_{+}\right|} \cdot 100
$$

onde $\left|\dot{I}_{-}\right|$e $\left|\dot{I}_{+}\right|$são respectivamente o módulo dos fasores correspondentes aos componentes de seqüência negativa e positiva da corrente.

Pretende-se simular o funcionamento de um relé de seqüência negativa desconectando a carga da rede quando o desequilíbrio for de $75 \%$. O grau de desequilíbrio é calculado em tempo real a partir dos valores eficazes (calculado a partir de um integrador com janela móvel) de seqüência positiva e negativa calculados pelas operações CD e AB respectivamente.

Uma vez calculado o grau de desequilíbrio, procede-se a uma comparação com um valor de set-point (no caso $75 \%$ ), quando os valores forem os mesmos a chave será acionada e a carga desconectada. Para esta implementação fez-se necessária a utilização de um circuito de bloqueio inicial do acionamento do relé durante um ciclo de modo a não promover um acionamento indesejado devido às condições inicias de cálculo do grau de desequilíbrio (tempo necessário ao Matlab para o cálculo do grau de desequilíbrio a partir dos valores obtidos pelo algoritmo proposto).

Para o teste de acionamento do relé, simulou-se o comportamento da carga conforme as seguintes características:

I. Sistema com carga equilibrada é submetido a um degrau de carga que caracteriza o sistema com um desequilíbrio $(X \%=50 \%)$ em $t=2 / 60 \mathrm{~s}$

II. Submete-se o sistema desbalanceado a um novo degrau de carga de forma a obter o máximo desequilíbrio $(X \%=100 \%)$ em $t=4 / 60 \mathrm{~s}$ 
A Fig.5.2-1 apresenta o comportamento da corrente da rede, do grau de desequilíbrio e do acionamento do relé em função do tempo.
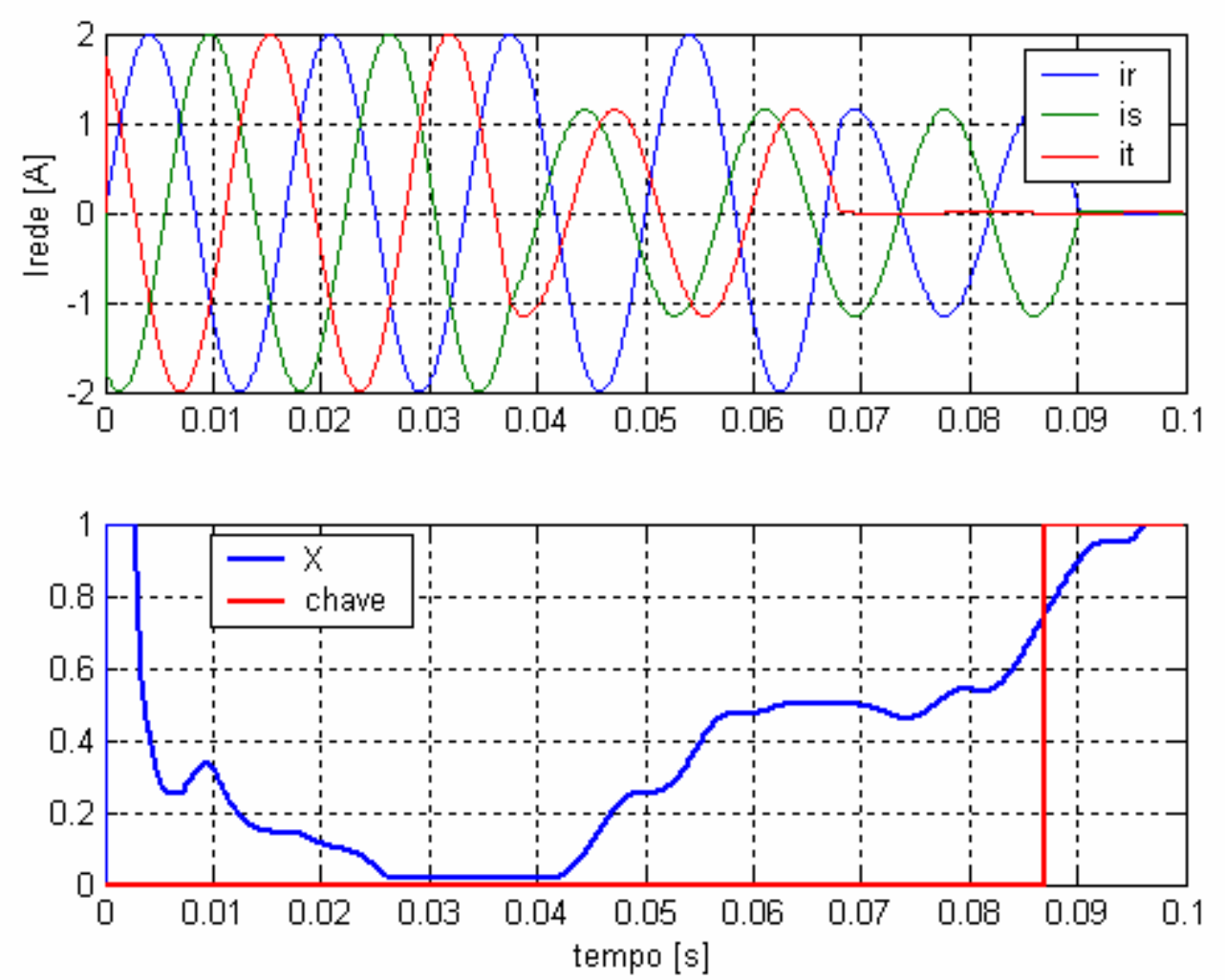

Fig.5.2-1 - Simulação Relé de Seqüência Negativa Comportamento da corrente da rede (gráfico superior) Comportamento do acionamento do relé (gráfico inferior)

Observa-se que a detecção e o desligamento foram realizados conforme a configuração estabelecida $(X>=75 \%)$. O atraso no chaveamento deve-se ao modelo de chave existente no Matlab (a chave apenas executa o comando de abrir após a corrente chegar a zero).

A seguir são apresentados os circuitos utilizados para a implementação do relé de seqüência negativa:

- o circuito geral (Fig. 5.2-2);

- o cálculo das seqüência positiva e negativa pelas operações CD e AB (Fig. 5.2-3);

- o cálculo das correntes eficazes (Fig. 5.2-4);

- o cálculo do grau de desequilíbrio e comparação com o set-point (Fig. 5.2-5). 


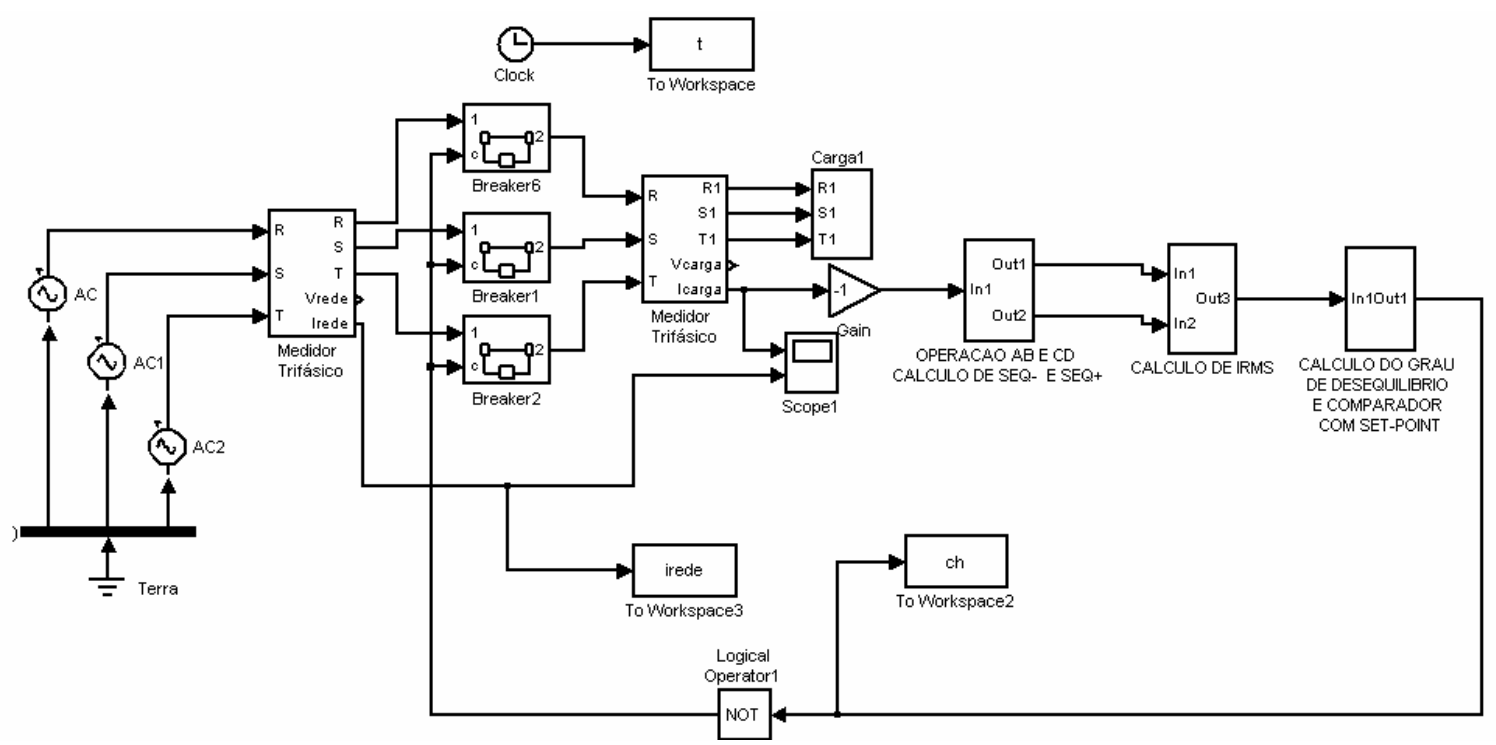

Fig. 5.2-2 - Circuito do relé de seqüência negativa simulado (Circuito Geral)

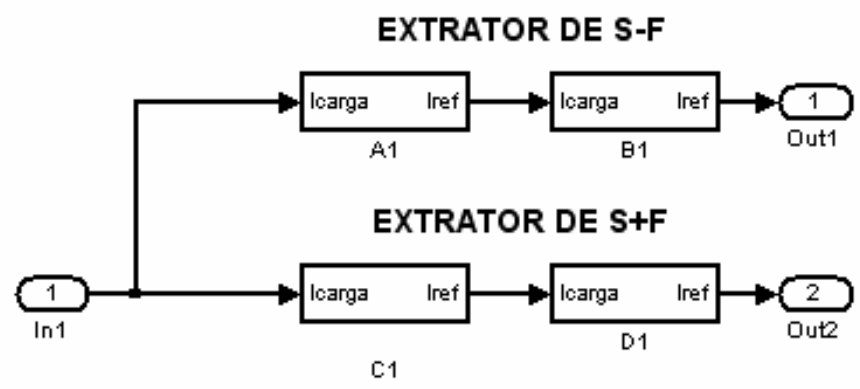

Fig. 5.2-3 - Circuito do relé de seqüência negativa simulado (cálculo das seqüência positiva e negativa)

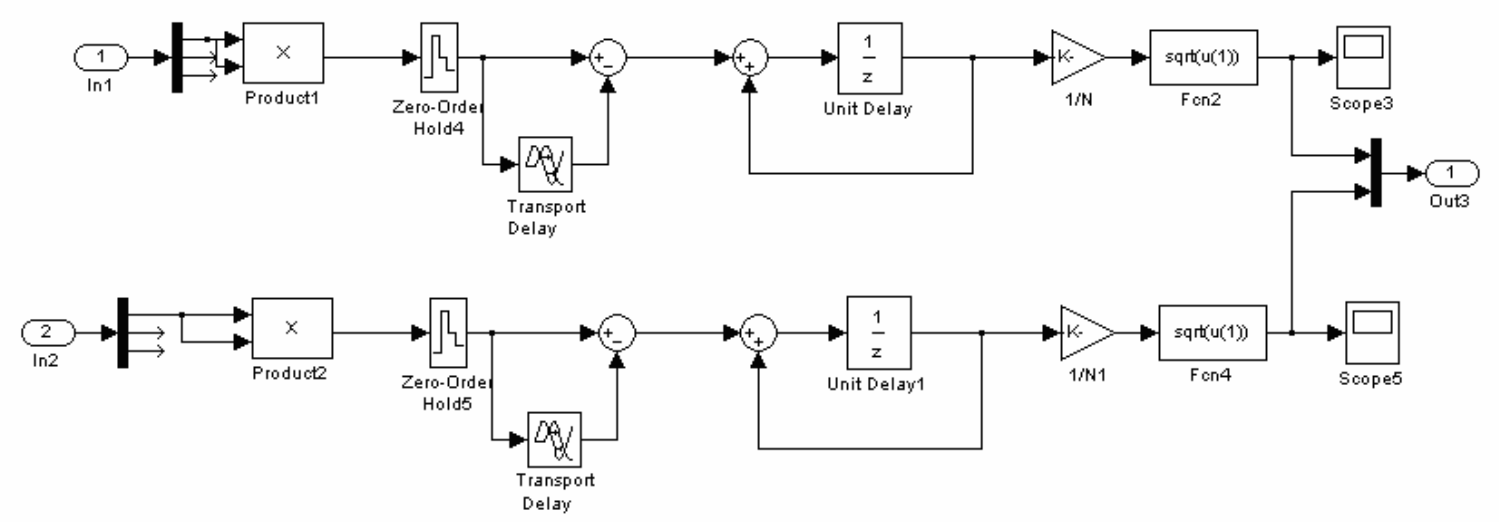

Fig. 5.2-4 - Circuito do relé de seqüência negativa simulado (cálculo das correntes eficazes) 


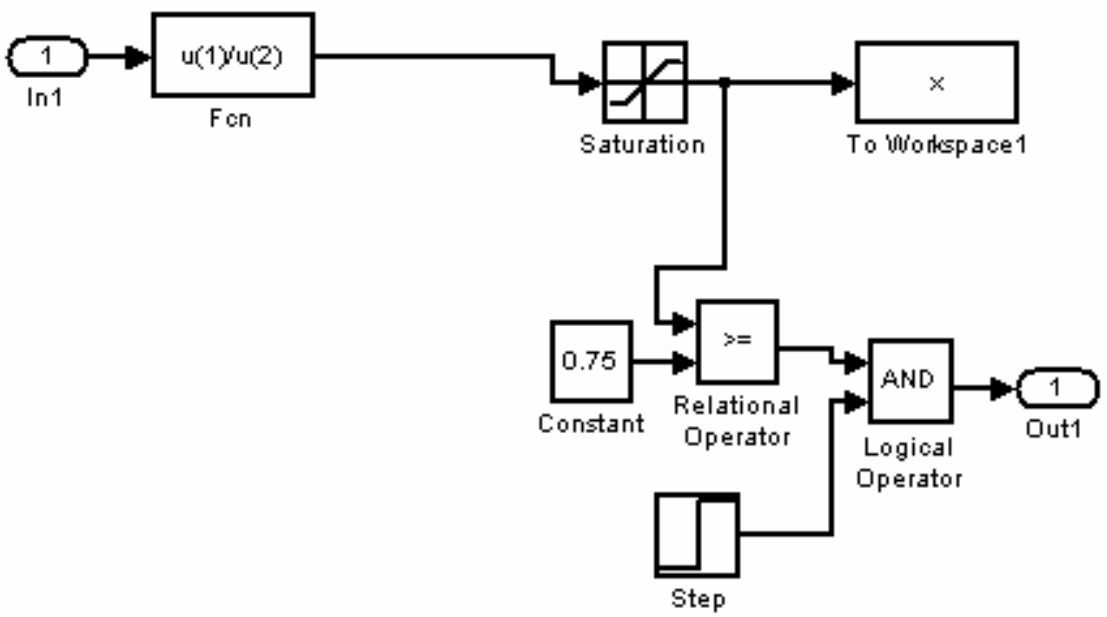

Fig. 5.2-5 - Circuito do relé de seqüência negativa simulado (cálculo do grau de desequilíbrio e comparação com set-point) 


\section{CONCLUSÕES E SUgESTÕES PARA A CONTINUIDADE DO TRABALHO}

Seguem abaixo as conclusões finais deste trabalho e as sugestões para a sua continuidade.

Os diferentes métodos de extração apresentados mostraram-se como uma alternativa viável e eficiente quando o objetivo é o cálculo da seqüência negativa, positiva e/ou harmônicos em tempo real. O fato de funcionarem como um filtro digital com tempo de atuação bem definido, a possibilidade de se trabalhar sem PLL, desde que a freqüência seja $60 \mathrm{~Hz}$, a operação direta com os sinais medidos, a baixa complexidade numérica que resulta um tempo de execução pequeno com convergência menor que um ciclo são características que os tornam atrativos para várias aplicações, no entanto, a influência da extração parcial dos harmônicos pares e da variação de ganho e fase quando da variação da freqüência devem ser objeto de análise de acordo com a aplicação a ser realizada.

A opção pela extração total ou parcial dos distúrbios no sistema (desequilíbrios e harmônicos) depende das características desejadas e da aplicação a ser desenvolvida. As análises efetuadas da resposta em regime, transitória e em freqüência fornecem ao projetista ferramenta para a escolha do método mais adequado à sua necessidade.

Seguem abaixo propostas para a continuidade do presente trabalho:

- aplicar os métodos propostos em diversos casos de aplicação e a seguir comparar seu desempenho com outros métodos conhecidos da literatura.

- aplicar os conceitos estudados nos métodos propostos para o cálculo de potências ativas e reativas;

- estudar o uso dos métodos propostos para geração de PLL comparando-os com outras técnicas disponíveis na literatura;

- estudar o uso dos métodos propostos quando da operação com inter e subharmônicos;

- ajustar o algoritmo implementado no DSP de modo a melhorar a resposta das operações propostas; 


\section{LISTA DE REFERÊNCIAS}

ABELlÁN,A; GARCERÁ,G.; BENAVENT,J. A New Control Method for Obtaining Reference Currents of Shunt Active Power Filters in Unbalanced and Non-Sinusoidal Conditions. In:Industrial Electronics- ISIE'99 Proceedings of the IEEE International Symposium on,Jul.1999. Anais eletrônicos. v.2.

AHN,S.U.; JARDINI,J.A.; MATAKAS JR.,L.; KOMATSU,W.; MASUDA,M.; SILVA,F.; GALASSI,M.; MARTINZ,F.; COPELIOVICT,H.; OLIVEIRA,M.A.; CAMARGO,J.; ZANETTI,E.; LIMA,E.; Implementação e testes da geração de referência e controle em um protótipo de restaurador dinâmico de tensão.In: VI SBQEE VI Seminário Brasileiro sobre Qualidade da Energia Elétrica, Belém, Pará, Brasil, Agosto, 2005.

AKAGI,H.; NABAE,A. The p-q Theory in Three-Phase Systems Under Non-Sinusoidal Conditions. ETEP, v.3, n.1, Jan. Feb. 1993.

AKAGI,H.; NABAE,A.; KANAZAWA,Y. Instantaneous reactive power compensators comprising switching devices without energy storage - IEEE Trans. Ind. Applicat, 1984

ANALOG DEVICES. ADMC401 EVALUATION KIT - todos os livros que fazem parte do kit de desenvolvimento, manuais de hardware e software;

BHAVARAJU,V.P.; ENJETI,P.N. Analysis and Design of an Active Power Filter for Balancing Unbalanced Loads. IEEE Transactions on Power Electronics, v.8, n.4, Oct. 1993.

BRITTAIN,J.C. Charles F. Scott : A Pioneer in Electrical Power Engineering. IEEE Industry Applications Magazine, p.6-8. Nov. Dec. 2002.

CHANG,G.W.; SHEE,T. A Comparative Study of Active Power Filter Reference Compensation Approaches. IEEE 2002 Power Engineering Society Summer Meeting, 21-25 July 2002. Anais, IEEE, v.2, p.1017-1021. 2002.

CHEN,C.C.; HSU,Y. A Novel Approach to the Design of a Shunt Active Filter for na Unbalanced Three-Phase Four Wire System under Nonsinusoidal Conditions. In:Power Engineering Society Summer Meeting,Jul.2000. Anais eletrônicos. v.2. p.1059.

CHOI,S.; LEE,W.; HYUN,D.; LEE, T. The Control system of the Active Power Filter Considering Power Factor in Unbalanced Load. In:Industry Applications Conference, Oct.2000. Anais eletrônicos. v.4. p.2123-2128.

CLARKE, Edith. Circuit Analysis of AC Power System, v.1, New York :John Wiley \& Sons, 1943.

CUTRI,R.; MATAKAS JR.,L. Reference Currents Determination Techniques For Load Unbalance Compensation. In: $7^{\circ}$ Congresso Brasileiro de Eletrônica de PotênciaCOBEP'03,Fortaleza,Set.2003. CD-ROM 
CUTRI,R.; MATAKAS JR.,L. A new instantaneous method for harmonics, positive and negative sequence detection for compensation of distorted currents with static converters using pulse width modulation. In: $11^{\circ} \mathrm{ICHQP} 11$ th International Conference on Harmonics and Quality of Power 2004, Lake Placid, New York, September, 2004.

CUTRI,R. Compensação de desequilíbrios de carga empregando conversor estático operando com modulação em largura de pulso. 2004. Dissertação (Mestrado) - Escola Politécnica,Universidade de São Paulo. São Paulo.

CUTRI,R.; MATAKAS JR.,L. A generalized instantaneous method for harmonics, positive and negative sequence detection/extraction. In: PESC 2007 (IEEE Power Electronics Specialists Conference) - Junho 17-21, Orlando, Flórida/USA

CZARNECKI,L.S. Power Factor Improvement of Three-Phase Unbalanced Loads with Non-Sinusoidal Supply Voltage. ETEP, v.3, n¹, Jan. Feb. 1993.

DECKMANN, S.M.; MARAFAO, F.P. Time based decompositions of voltage, current and power functions - Harmonics and Quality of Power, 2000. Proceedings. Ninth International Conference on Volume 1, Issue , 2000 Page(s):289 - 294 vol.1

DIXON,J.W.; GARCIA,J.; MORÁN,T.L. A Control System for a Three Phase Active Power Filter which Simultaneously Compensates Power Factor and Unbalanced Loads. IEEE Transactions on Industrial Electronics, v.42, Issue:6, p.636-641. Dec. 1995.

FERRERO,A. Unbalancing and Distorting Loads : Can They Be Detected and Their Detrimental Effect Be Measured ?. In:Harmonics and Quality of Power , Procceedings $8^{\text {th }}$ International Conference on,Oct.1998. Anais eletrônicos. v.2. p.809-813.

Fortescue,C.L. Method of symmetrical co-ordinates applied to the solution of polyphase networks. In:34th Annual Convention of the America Institute of Electrical Engineers. Atlantic City, N.J., June. 1918.

HAFNER,J.; AREDES,M.;HEUMANN, K.; "A shunt active filter applied to high voltage distribution lines," IEEE Trans. Power Delivery, vol. 12, pp. 266-272, Jan. 1997.

HOCHGRAF,C.; LASSETER,R. Statcom Controls for Operation with Unbalanced Voltages. IEEE Transactions on Power Delivery, v.13. n.2, Apr. 1998.

IEEE 519-1992 IEEE Recommended Practices and Requirements for Harmonic Control in Electrical Power Systems - Institute of Electrical and Electronics Engineers / 01-May1992 - ISBN: 1559372397

KIM,H.; BLAABJERG,F; JENSEN,B.B.; CHOI,J. Instantaneous Power Compensation in Three-Phase Systems by Using p-q-r theory. In:Power Electronics Specialists Conference,2001-PESC'2001- IEEE 32 ${ }^{\text {nd }}$ Annual,June.2001. Anais eletrônicos. v.2. 
p.478-485.

$\mathrm{KIM}, \mathrm{H}$. Control an Active Filter in Unbalanced Non- sinusoidal Source Voltages. In:Power Conversion Conference Proceedings of.,Osaka,Apr.2002. Anais eletrônicos. v.2. p.831-836. Acesso restrito disponível em:<periódicos IEEE-Capes>.

LEDWICH,G.; GEORGE,T.A. Using Phasors to analize power system negative phase sequence voltages caused by unbalanced loads. IEEE Transactions in Power Systems, v.9, n.3, Aug. 1994.

LIN,C.E.; CHEN,C.L.; HUANG,C.L. Calculating Approach and Implementation for active Filters in Unbalanced Three-Phase System using Synchronous Detection Method. In:Industrial Electronics,Control,Instrumentation and Automation - 'Power Electronics and Motion Control', Proceedings of the 1992 International Conference on,Nov.1992. Anais eletrônicos. v.1. p.374-380.

MARAFÃO F.P.; DECKMANN S.M.; POMILIO J.A.; MACHADO R.Q.; A SoftwareBased PLL Model: Analysis And Applications - CBA 2004 - XV Congresso Brasileiro de Automática. Gramado-Rio Grande do Sul. .Setembro 2004.

MARQUES,G.D. A Comparison of Active Filters Control Methods in Unbalanced and Non-sinusoidal Conditions. In:Industrial Electronics Society - IECON'98 Proceedings of the $24^{\text {th }}$ Annual Conference of the IEEE,Aug.Sep.1998. Anais eletrônicos. v.1. p.444449. Acesso restrito disponível em:<periódicos IEEE-Capes>.

MATAKAS JR.,L. Conexão Paralela de Conversores Estáticos do Tipo Fonte de Tensão Sem Transformador. 1998. Tese (Doutorado) - Escola Politécnica,Universidade de São Paulo. São Paulo.

MATAKAS JR.,L.; MARTINZ F.O.; GIARETTA A.R.; GALASSI M.; KOMATSU W. Uma Abordagem Gráfica Para Um Algoritmo De PII Baseado Em Seqüência Positiva CBA2006 - $16^{\circ}$ Congresso Brasileiro de Automática, Bahia, Outubro 2006.

MATSUI,M.; FUKAO,T. A Detecting Method for Active-Reactive-Negative Sequence Powers ans its Application. IEEE Transactions on Industry Applications, v.26, n.1, Jan. Feb. 1990.

MCGRANAGHAM,M. Active Filter Design and Specification for Control of Harmonics in Industrial and Commercial Facilities. Electrotek Concepts,Inc. Knoxville TN, USA. Disponível em:<http://www.dranetz-bmi.com/pdf/activeFilter.pdf>. Acesso em:2003.

NABAE,A.; NAKANO,H.; TOGASAWA,S. An Instantaneous Distorcion Current Compensator Without Any Coordinate Transformation. In:International Power Electronics Conference-IPEC'95,Yokohama,1995. p.1651-1655. 1995.

NIKOLAENKO,V.G. Optimal Balancing of Large Unbalanced Loads Using Shunt Compensators. In:Harmonics and Quality of Power , Procceedings $8^{\text {th }}$ International Conference on,Oct.1998. Anais eletrônicos. v.1. p.537-542. 
ONS - Operador Nacional do Sistema Submódulo 2.2 - Padrões de desempenho da Rede Básica - (24/12/2002) Resolução n 791/02 - ANEEL - obtido no endereço na Internet: http://www.ons.org.br

PENTEADO JR.,A.A. Compensadores estáticos para desequilíbrios : um procedimento de especificação e de análises das interferências no sistema elétrico. 1985. Tese (Doutorado) - Escola Politécnica,Universidade de São Paulo. São Paulo.

ROBBA,J. et al. Introdução a Sistemas Elétricos de Potência. 2ed. São Paulo:Edgard Blucher, 1996.

SANTANU S; P. K. MAHARANA AND V.K. AGARWAL Phase-Locked Loop Design for Flexible Mode Tracking Control System Group. ISRO SATELLITE CENTRE, Baogalorc. India. Anais eletrônicos IEEE XPLORE 2003 - <acessado em outubro de 2007>.

SENINI,S.; WOLFS,P. Hybrid Active Filter for Harmonically Unbalanced Three Phase Three Wire Railway Traction Loads. IEEE Transactions on Power Electronics, v.15, n.4, July. 2000.

SENINI,S.T.; WOLFS,P.J. Novel Topology of Unbalanced Load in Single Phase Electric Traction Systems. In:Power Electronics Specialists Conference,2001-PESC'2002IEEE 33 ${ }^{\text {rd }}$ Annual,June.2002. Anais. v.3. p.1208-1212. 2002.

VERDELHO,P.; MARQUES,G.D. Design and Performance of an Active Power Filter and Unbalanced Current Compensator. In:Industrial Electronics,Control and Instrumentation,Sep.1994. IECON'94,20 ${ }^{\text {th }}$ International Conference on. Anais eletrônicos. 1994. v.1. p.422-427.

WATANABE,E.; AREDES,M. Teoria de Potência Ativa e Reativa Instantânea e Aplicações - Filtros Ativos e FACTS". Tutorial in the CBA'98 (XII Brazilian Automatic Control Conference), Uberlândia, Brazil, Sep.14-18, 1998.

WATANABE,E.; AREDES,M.; AKAGI,H. The p-q theory for active filter control : some problems and solutions. In:XIV Congresso Brasileiro de Automática-CBA,2002.

WILLEMS,J.L. Current Compensation in Three-Phase Power Systems. ETEP, v.3, n.1, Jan. Feb. 1993.

ZHANG, G.; XU, Z. A New Real-time Negative and Positive Sequence Componentes Detecting Method Based on Space Vector. In:POWER ENGINEERING SOCIETY WINTER MEETING 2001,Jan.-Feb.2001. Anais eletrônicos. IEEE, v.1. p.275-280. 


\section{APÊNDICE A - MÉTODO PROPOSTO DE EXTRAÇÃO APLICADO A UM SINAL COM SEQUÊNCIA ZERO}

A eq. (3.2), utilizada para obtenção dos sinais de seqüência zero, pode ser reescrita no domínio do tempo eq. (AP-1).

$$
\left[\begin{array}{l}
s_{r 0}(t) \\
s_{s 0}(t) \\
s_{t 0}(t)
\end{array}\right]=\frac{1}{3} \cdot\left[\begin{array}{lll}
1 & 1 & 1 \\
1 & 1 & 1 \\
1 & 1 & 1
\end{array}\right] \cdot\left[\begin{array}{l}
s_{r}(t) \\
s_{s}(t) \\
s_{t}(t)
\end{array}\right]
$$

Assim, pode-se antes de aplicar as operações propostas (cap. 3) extrair o sinal de sequência zero existente.

Caso a sequência zero não seja extraída antes da aplicação das operações (A, $\mathbf{B}$, C, D) ela será processada pelas operações, podendo ser extraída ou não dependendo de sua ordem e da operação utilizada (A, B, C, D).

A demonstração matemática desta constatação é feita adotando-se um sinal trifásico contendo um componente harmônico genérico de seqüência zero e calculando-se o sinal extraído de acordo com a operação proposta.

Assim, sejam os sinais instantâneos $s_{\text {rho }}(t), s_{\text {sho }}(t)$ e $s_{\text {tho }}(t)$ representados na forma exponencial respectivamente nas eqs.(AP-2), (AP-3) e (AP-4).

$$
\begin{aligned}
& S_{r h 0}(t)=\operatorname{Re}\left(S_{0} \cdot e^{j\left(h \omega t+\phi^{h 0}\right)}\right) \\
& S_{s h 0}(t)=\operatorname{Re}\left(S_{0} \cdot e^{j\left(h \omega t+\phi^{h 0}\right)}\right) \\
& S_{t h 0}(t)=\operatorname{Re}\left(S_{0} \cdot e^{j\left(h \omega t+\phi^{h 0}\right)}\right)
\end{aligned}
$$


AP.1 Demonstração matemática da operação A aplicada a um sinal contendo sequência zero

A seguir descreve-se o cálculo de $s_{r-}(t)$ (eq.(AP.1-1)) substituindo os valores dados pelas eqs. (AP-2), (AP-3), (AP-4) na eq.(3.6).

$$
S_{r-}(t)=\operatorname{Re}\left(\frac{S_{0}}{3} \cdot e^{j\left(h \omega t+\phi^{h 0}\right)}-\frac{S_{0}}{3} \cdot e^{j\left(h \omega t+\phi^{h 0}-h \cdot \frac{\pi}{3}\right)}+\frac{S_{0}}{3} \cdot e^{j\left(h \omega t+\phi^{h 0}-h 2 \frac{\pi}{3}\right)}\right)
$$

Os sinais $\mathrm{s}_{\mathrm{s}-(}(\mathrm{t})$ e $\mathrm{s}_{\mathrm{t}-(}(\mathrm{t})$ seguem o mesmo procedimento e são idênticos a $\mathrm{s}_{\mathrm{r}-\mathrm{(}}(\mathrm{t})$.

\section{AP.2 Demonstração matemática da operação B aplicada a um sinal contendo sequência zero}

A seguir descreve-se o cálculo de $s_{r-}(t)$ (eq.(AP.2-1)) substituindo os valores dados pelas eqs. (AP-2), (AP-3), (AP-4) na eq.(3.7).

$$
\begin{aligned}
& S_{r-}(t)=\operatorname{Re}\left(\frac{S_{0}}{3} \cdot e^{j\left(h \omega t+\phi^{h 0}\right)}-\frac{S_{0}}{6} \cdot e^{j\left(h \omega t+\phi^{h 0}\right)}-\frac{S_{0}}{6} \cdot e^{j\left(h \omega t+\phi^{h 0}\right)}\right. \\
& \left.+\frac{\sqrt{3}}{6} \cdot S_{0} \cdot e^{j\left(h \omega t+\phi^{h 0}-h \cdot \frac{\pi}{2}\right)}-\frac{\sqrt{3}}{6} \cdot S_{0} \cdot e^{j\left(h \omega t+\phi^{h 0}-h \cdot \frac{\pi}{2}\right)}\right)
\end{aligned}
$$

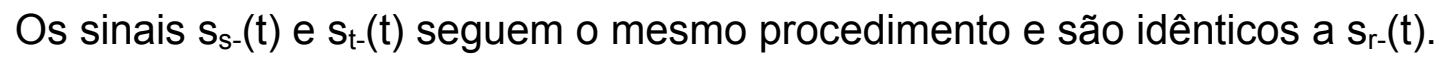

De forma análoga, as demonstrações dos itens AP.1 e AP.2 valem respectivamente para as operações $\mathbf{C}$ e $\mathbf{D}$. 


\section{AP.3 RESPOSTA EM REGIME}

A partir dos resultados dos itens AP.1 e AP.2, apresentam-se na Tabela X o ganho de amplitude e a defasagem relativos ao uso das operações A, B, C e D para um sinal de seqüência zero.

TABELA X - Ganho de amplitude (p.u.) e fase (graus) das operações A, B, C, D para harmônicas pares e ímpares de seqüência zero.

\begin{tabular}{|c|c|c|c|c|}
\hline Operação & $\mathrm{A}$ & $\mathrm{B}$ & $\mathrm{C}$ & $\mathrm{D}$ \\
\hline $1^{\circ}$ seq0 & 0 & 0 & 0 & 0 \\
\hline $2^{\circ}$ seq0 & $\frac{2}{3} \sqrt{60}$ & 0 & $\frac{2}{3} \sqrt{60}$ & 0 \\
\hline $3^{\circ}$ seq0 & $1 \sqrt{0}$ & 0 & $1 \sqrt{0}$ & 0 \\
\hline $4^{\circ}$ seq0 & $\frac{2}{3} \sqrt{-60}$ & 0 & $\frac{2}{3} \sqrt{-60}$ & 0 \\
\hline $5^{\circ}$ seq0 & 0 & 0 & 0 & 0 \\
\hline $6^{\circ}$ seq0 & $\left.\frac{1}{3}\right) \overline{0}$ & 0 & $\frac{1}{3} \sqrt{0}$ & 0 \\
\hline $7^{\circ}$ seq0 & 0 & 0 & 0 & 0 \\
\hline $8^{\circ}$ seq0 & $\frac{2}{3} \sqrt{60}$ & 0 & $\frac{2}{3} \sqrt{60}$ & 0 \\
\hline $9^{\circ}$ seq0 & $1 \sqrt{0}$ & 0 & $1) \sqrt{0}$ & 0 \\
\hline $10^{\circ}$ seq0 & $\left.\frac{2}{3}\right)-60$ & 0 & $\frac{2}{3} \sqrt{-60}$ & 0 \\
\hline $11^{\circ}$ seq0 & 0 & 0 & 0 & 0 \\
\hline $12^{\circ}$ seq0 & $\left.\frac{1}{3}\right) 0$ & 0 & $\left.\frac{1}{3}\right) 0$ & 0 \\
\hline
\end{tabular}

As operações A e C apresentam o mesmo ganho e fase para harmônicos de ordem $(2+6 n),(3+6 n),(4+6 n)$ e $(6+6 n)(n=0,1,2, \ldots)$ sendo que para outras ordens ou operações o ganho é nulo. 


\section{AP.4 Análise da influência da freqüência do sinal de Seqüência Zero}

A fim de analisar a influência da variação da freqüência na aplicação das operações A, B, C, D em sinal que contenham componentes de seqüência zero buscou-se verificar o comportamento das operações (baseadas no período fixo de $1 /(60 \mathrm{~Hz})$ ) com um sinal de freqüência compreendida entre $50 \mathrm{~Hz}$ e $70 \mathrm{~Hz}$ (passo de $1 \mathrm{~Hz}$ ) (Figs. AP-1).

Observa-se na Fig. AP-1 que, dependendo da ordem do harmônico de seqüência zero, as quatro operações apresentam grandes alterações de fase e amplitude para uma grande faixa de variação $(50$ a $70 \mathrm{~Hz})$ do sinal de entrada.
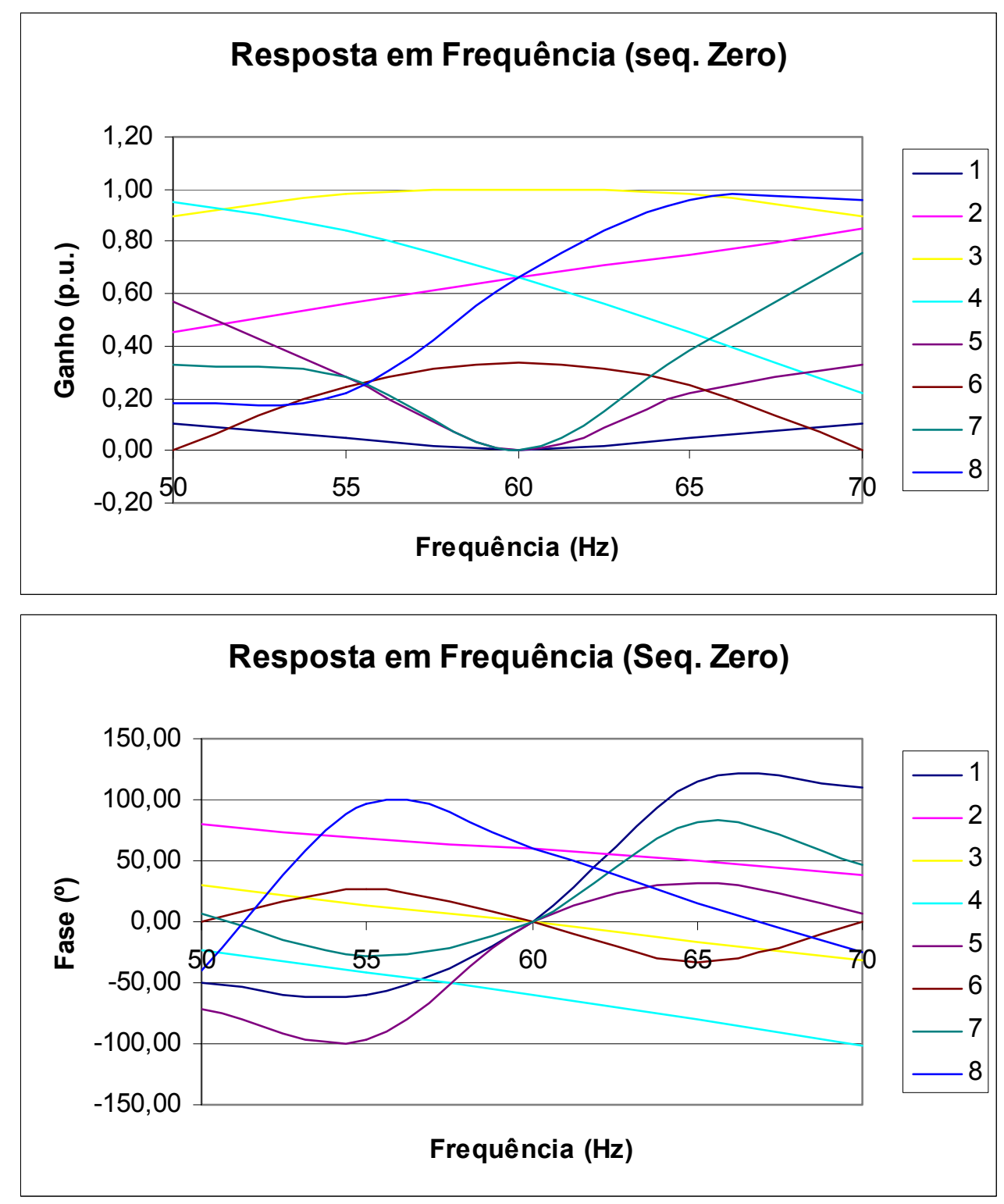

Fig. AP-1 - Operação A - Resposta em Freqüência para vários harmônicos de seqüência zero. Acima: ganho Abaixo:fase 
Assim, observa-se que o ganho (amplitude e defasagem), apresentado pelas quatro operações ao se aplicarem sinais com seqüência zero, é dependente da ordem do harmônico, da operação e da freqüência fundamental. Sugere-se assim que a seqüência zero seja extraída dos sinais originais através da eq. (AP-1), antes de se aplicarem quaisquer das quatro operações. 


\section{APÊNDICE B - IMPLEMENTAÇÃO DO BUFFER}

Cada operação (A, B, C, D) realizada demanda um atraso de tempo condizente com o deslocamento imposto em cada operação (capítulo 3), necessitando assim de um sistema de armazenamento (buffer).

$\mathrm{Na}$ simulação numérica, via Matlab, utilizou-se um bloco de atraso conforme disposto na Fig.B-1. No começo da simulação, o bloco fornece a saída de acordo com o valor inicial pré-definido (no caso adotou-se condição inicial nula) até que o tempo de simulação exceda o tempo de atraso definido. Os resultados da simulação numérica do armazenamento para um atraso de $60^{\circ}$, avanço de $60^{\circ}$ (atraso de $120^{\circ}$ com sinal negativo) e atraso de $90^{\circ}$ são apresentados na Fig.B-2.

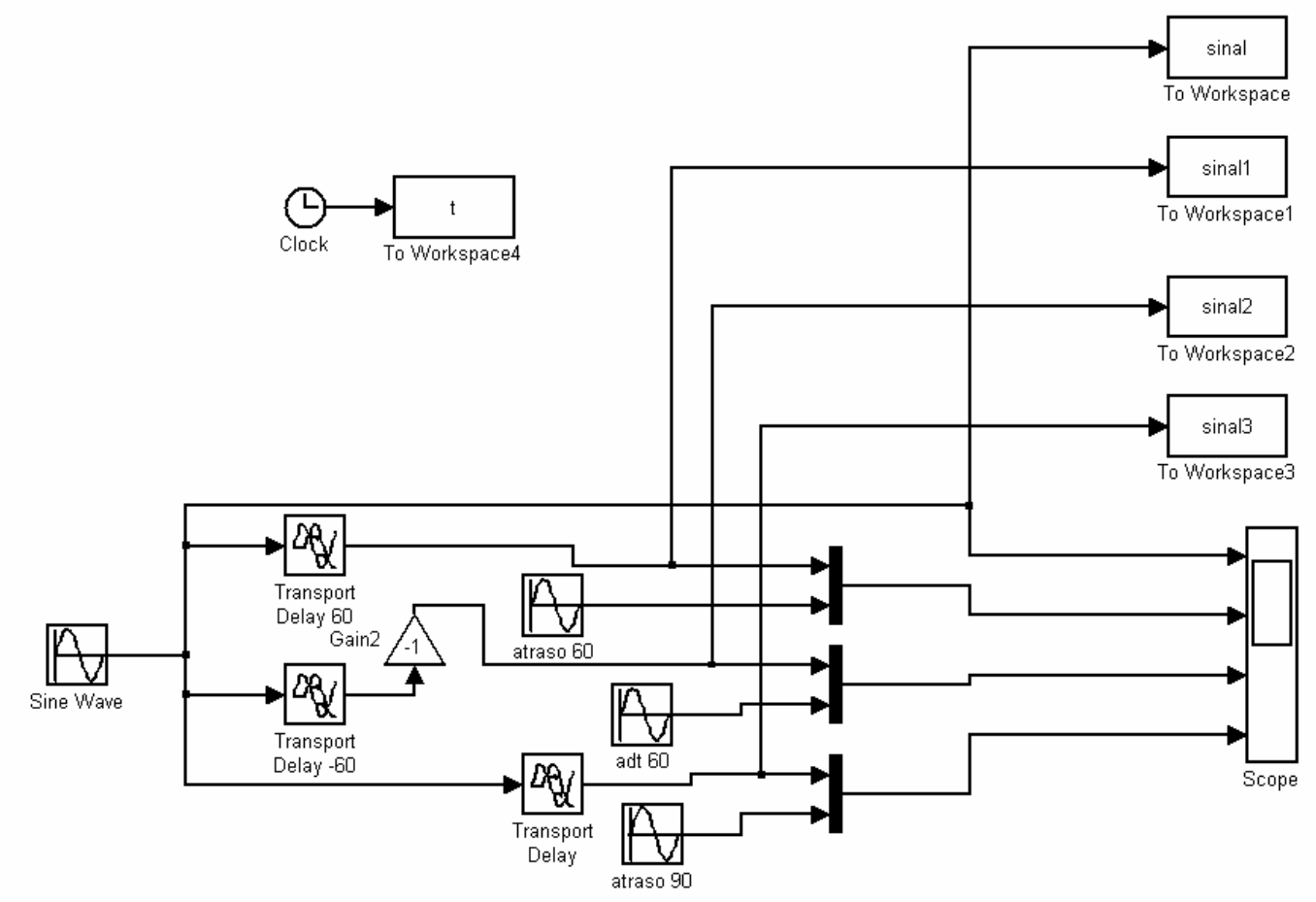

Fig.B-1 - Circuito Teste do Buffer (Simulação) 

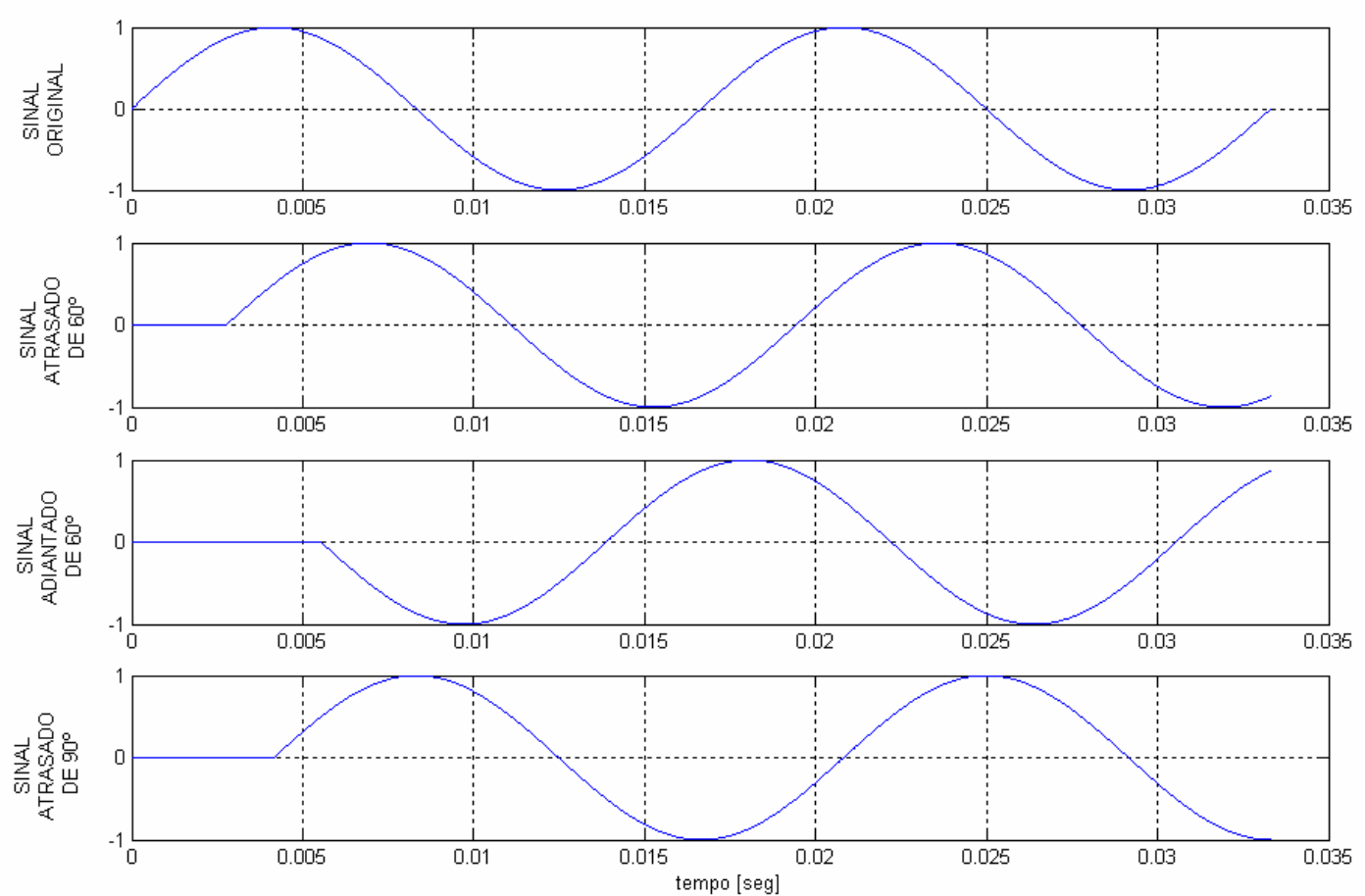

Fig.B-2 Sinais Bufferizados (Simulação)

(de cima para baixo)

sinal original / sinal atrasado de $60^{\circ} /$ sinal adiantado de $60^{\circ} /$ sinal atrasado de $90^{\circ}$

Para a implementação do buffer no DSP (ADMC-401- Fig.B-3), foi utilizado o mesmo princípio da simulação numérica.

FUNCTIONAL BLOCK DIAGRAM

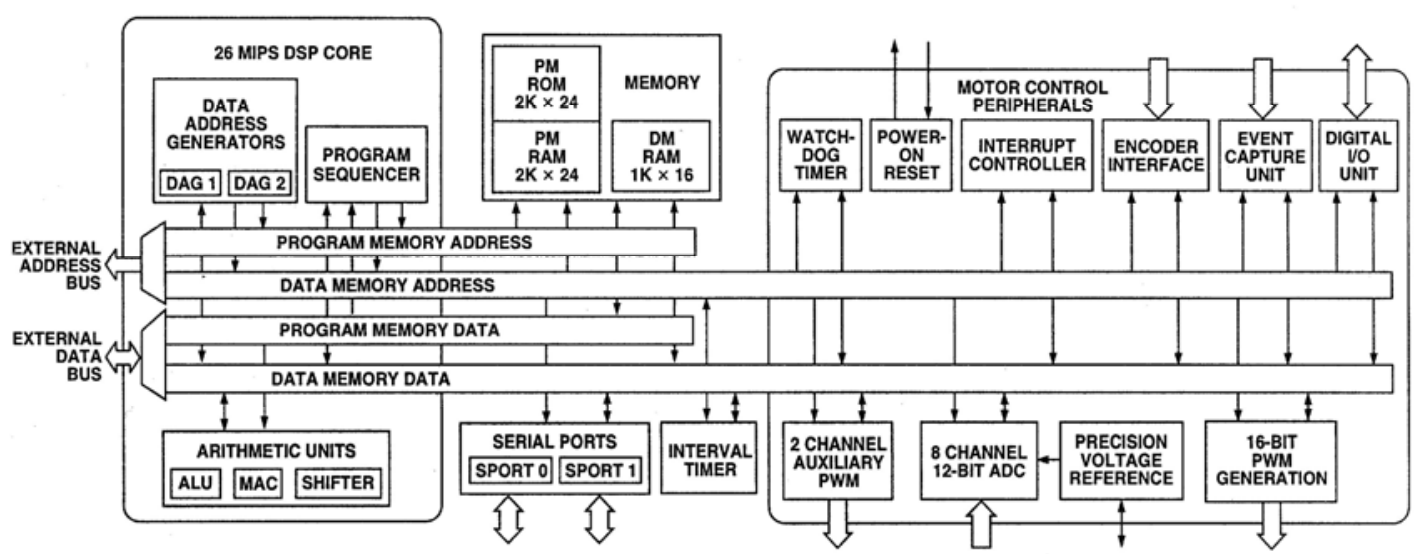

Fig.B-3 - Diagrama de Blocos do ADMC-401 
Conforme o capítulo 3 , o atraso de $90^{\circ}$ foi implementado armazenando-se as últimas N/4 amostras medidas, o avanço de $90^{\circ}$ foi obtido através da multiplicação dos sinais atrasados de $90^{\circ}$ por -1 . O atraso de $60^{\circ}$ foi obtido armazenando-se as últimas N/6 amostras medidas e o avanço de $60^{\circ}$ foi obtido utilizando-se as últimas $\mathrm{N} / 3$ amostras medidas, equivalentes a um atraso de $120^{\circ}$, com sinal negativo ( $\mathrm{N}$ é igual ao número de amostras por ciclo da rede na freqüência fundamental). Assim, observa-se que o maior número de amostras que devem ser armazenadas é de N/3, desta forma, implementou-se um buffer circular com esta dimensão de modo a extrair os valores necessários apenas posicionando-se adequadamente o ponteiro (Fig.B-4).

As operações somente são calculadas após inicialmente terem sido armazenadas N/3 amostras, sendo que a cada novo cálculo o valor armazenando no buffer é renovado $\left(\mathrm{n}_{\mathrm{i}-1} \leftarrow \mathrm{n}_{\mathrm{i}}\right)$ e a partir daí calcula-se novamente as operações.

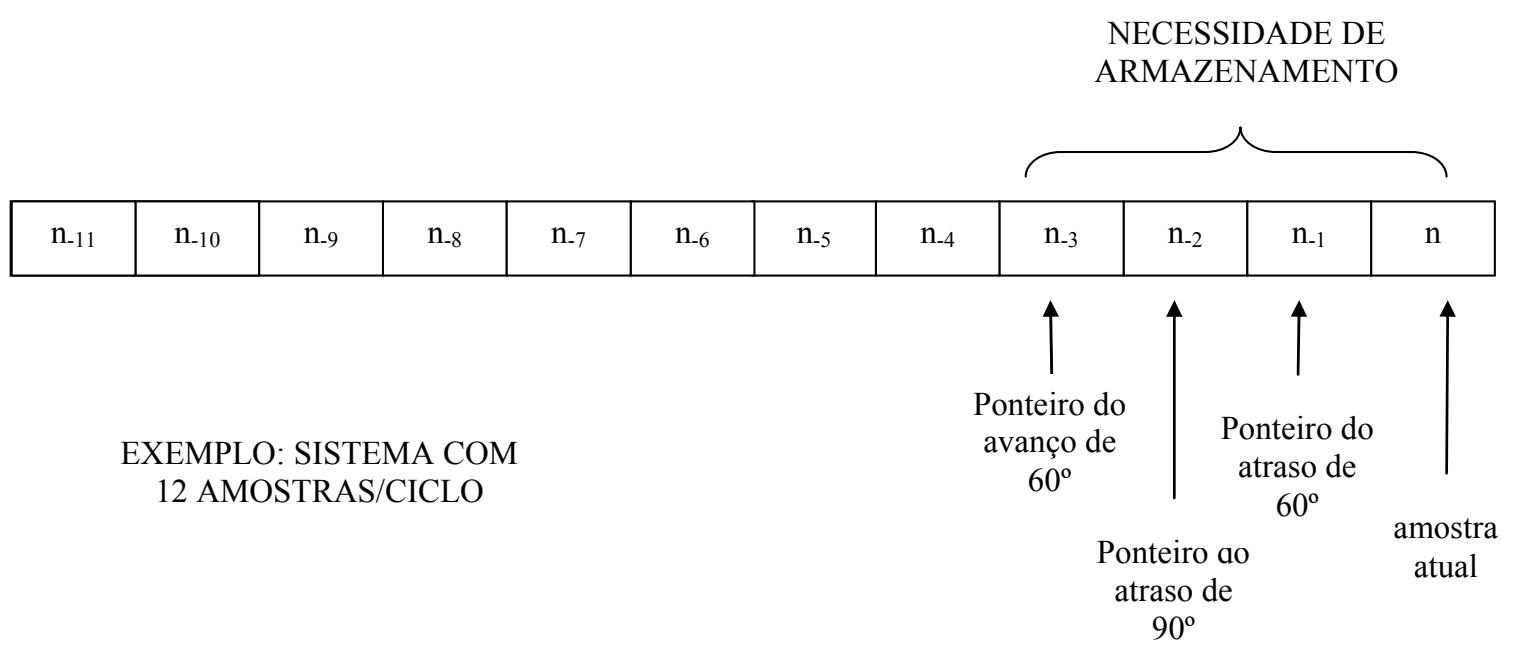

Fig.B-4 - Buffer digital 


\section{APÊNDICE C - CIRCUITOS SIMULADOS}

A seguir são apresentados os circuitos utilizados para o estudo dos métodos propostos bem como para a simulação dos exemplos constantes no capitulo 5 .

São apresentados:

- circuito-teste referente a todas as operações (Figura C-1)

- circuito simulado operação A (Figura C-2)

- circuito do filtro ativo simulado (compensador completo) (Figura C-3)

- circuito do conversor utilizado no filtro ativo simulado (parte 1) (Figura.C-4a )

- circuito do conversor utilizado no filtro ativo simulado (parte 2) (Figura.C-4b )

- malha de tensão utilizada no filtro ativo simulado (Figura C-5)

- malha de corrente utilizada no filtro ativo simulado (Figura C-6) 
No esquema da Fig. C-1, deve-se ler A no lugar de A1 e B no lugar de B1, o índice foi colocado automaticamente devido a restrição interna do Matlab quanto a duplicidade de nomes. Ainda na mesma figura, o bloco "Discrete 3 phase programable source" é um gerador de sinais trifásico programável em amplitude, freqüência, fase, ordem e seqüência.

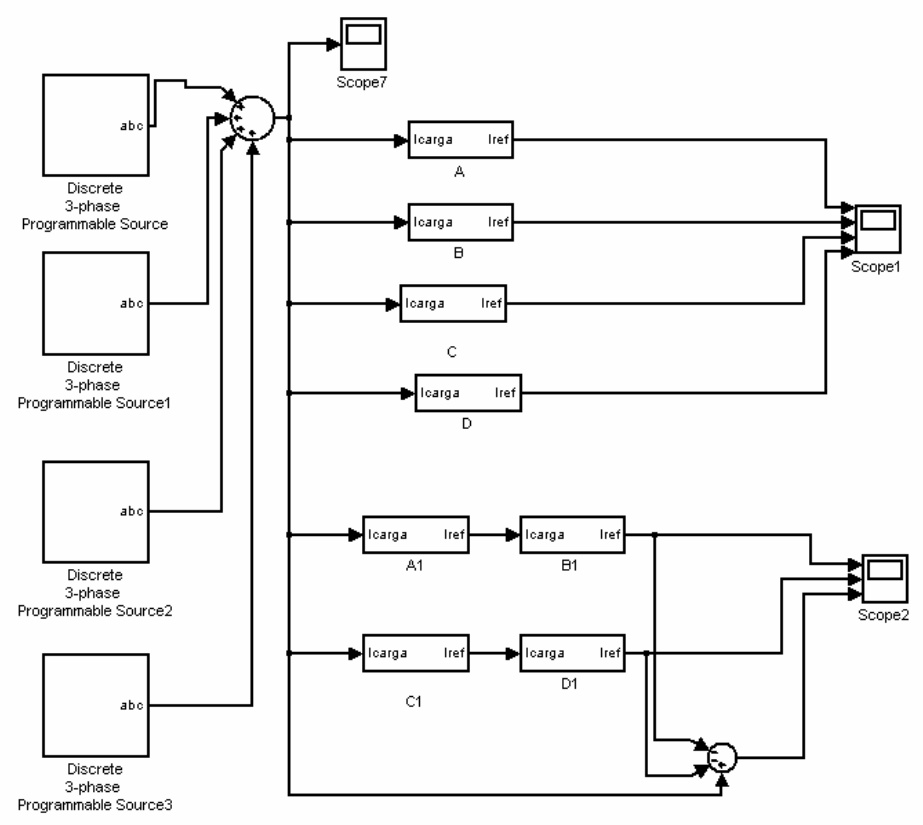

Fig. C-1 - Circuito-teste referente a todos os métodos

Na Fig.C-2 apresenta-se em detalhe o bloco A apresentado na Fig.C-1, que implementa a operação A proposta. Os blocos subsystem1,2 e 3 são blocos de atraso semelhantes ao já apresentado na Fig. B-2 e visam implementar as operações necessárias ao cálculo do sinal extraído da eq.(3.6). 


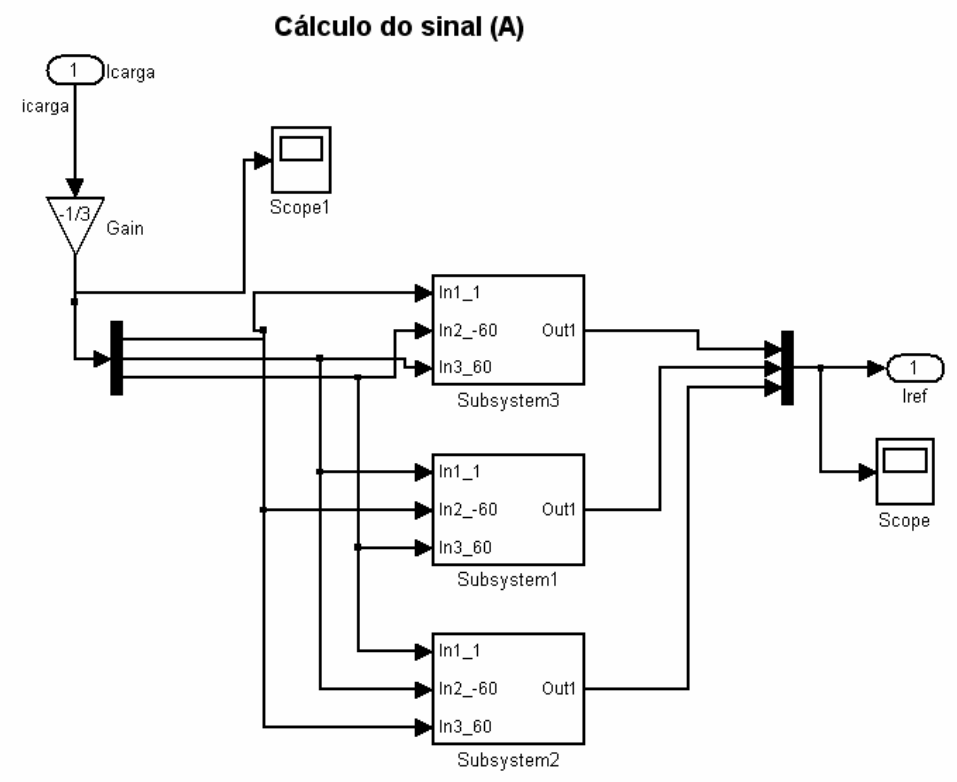

Fig. C-2 - Circuito simulado método A

Nas Figs. C-3, C-4, C-5 e C-6 a seguir, são apresentados respectivamente o circuito completo do Filtro Ativo simulado no capítulo 5, o bloco do conversor, a malha de tensão e a malha de corrente.

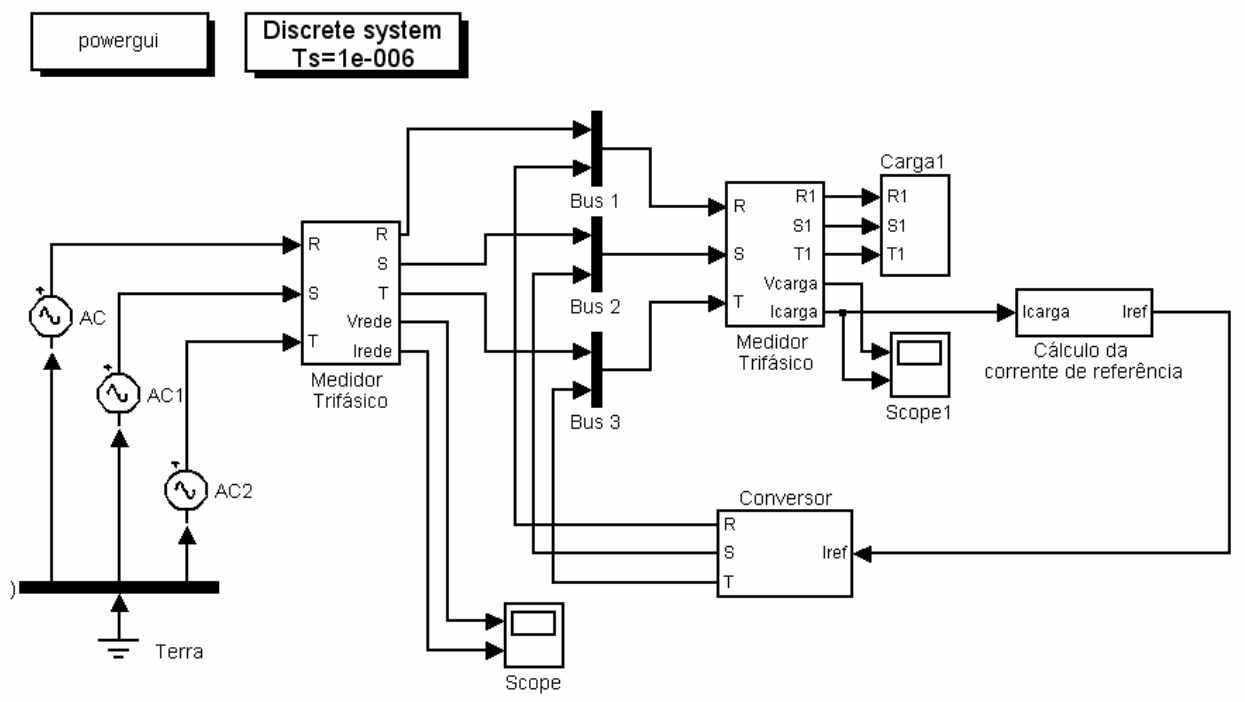

Fig. C-3 - Circuito do filtro ativo simulado (compensador completo) 


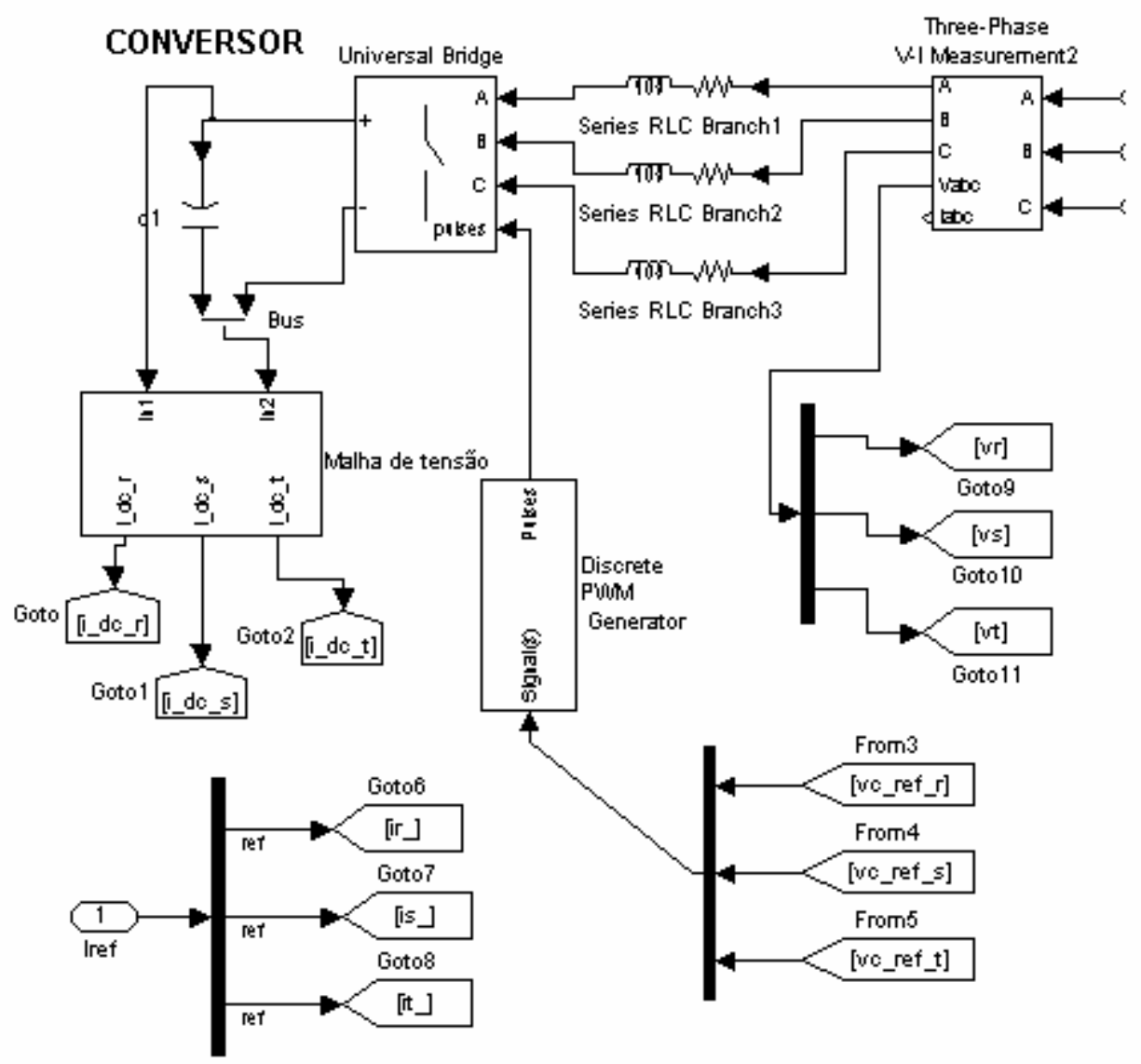

Fig.C-4a - Conversor (parte 1) 


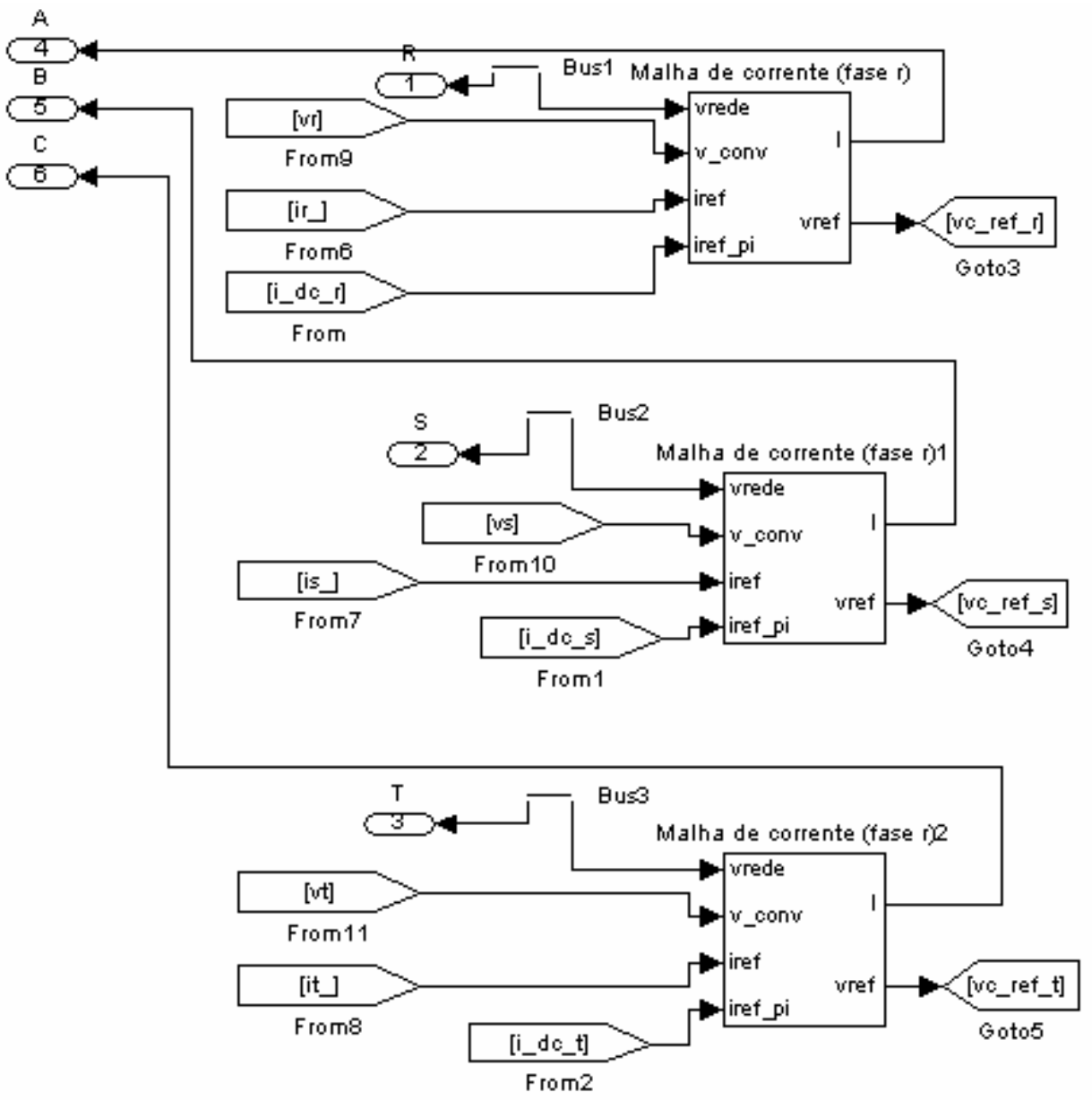

Fig.C-4b - Conversor (parte 2)

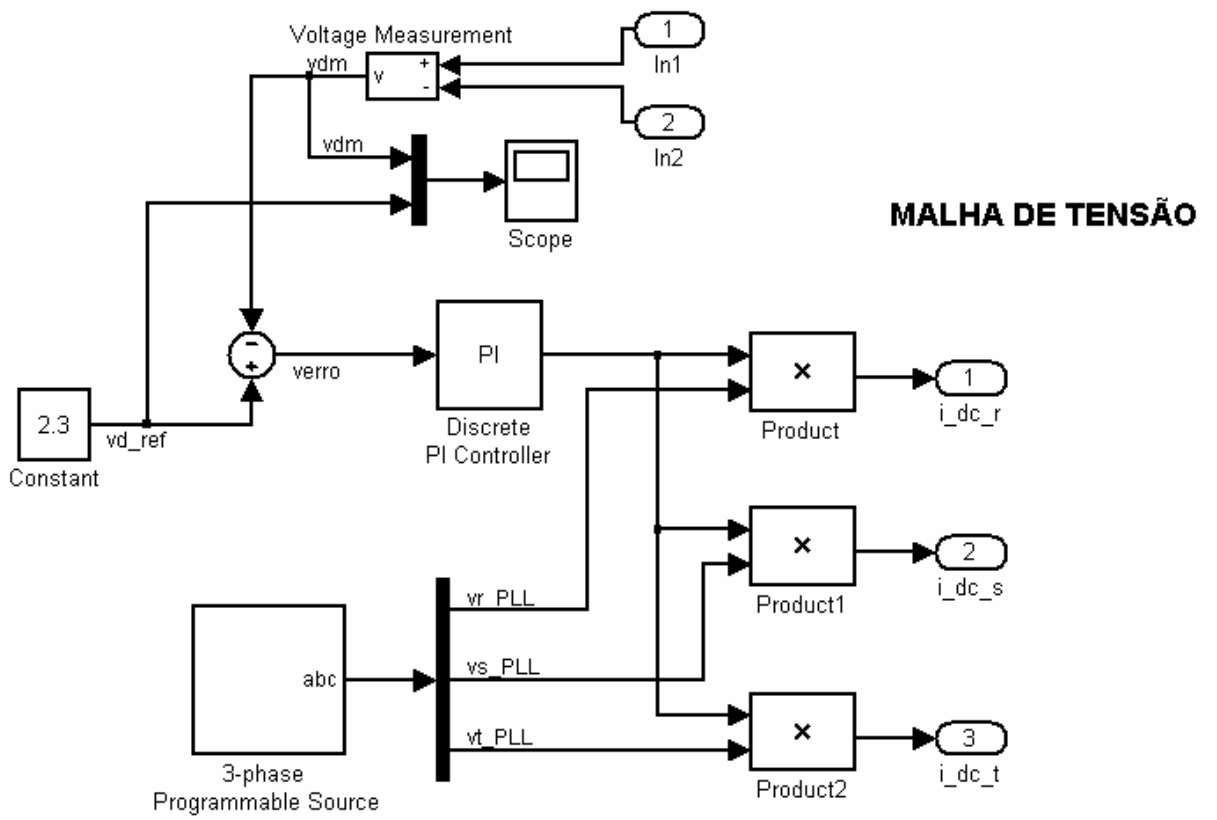

Fig.C-5 - Malha de tensão

Método de extração em tempo real de seqüência positiva, negativa e/ou harmônicos. 


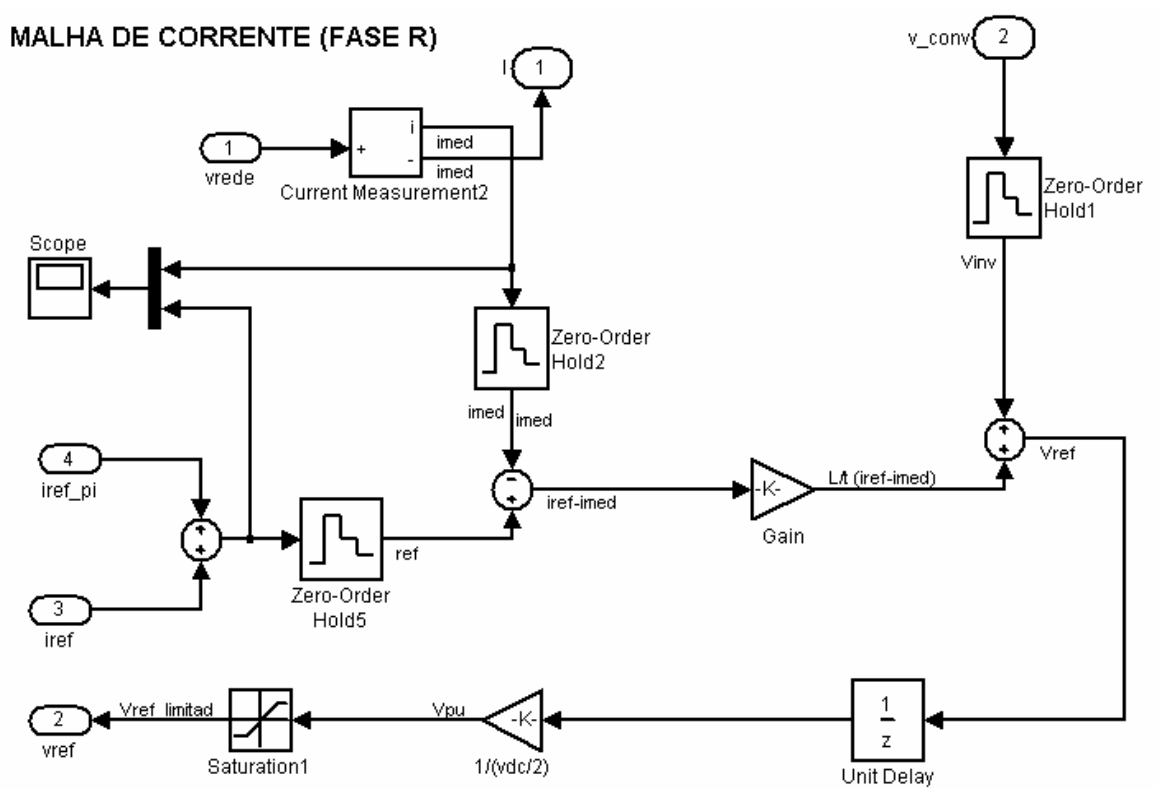

Fig.C-6 - Malha de corrente 